\title{
A Biphasic Multiscale Investigation of the Biomechanical Microenvironment of Articular Cartilage
}

\author{
by
}

\author{
Abdul R. Khan, B.Eng.
}

A thesis submitted to the Faculty of Graduate and Postdoctoral Affairs in partial fulfillment of the requirements for the degree of

Master of Applied Science

in

Mechanical Engineering

Carleton University

Ottawa, Ontario

(C) 2018, Abdul R. Khan 


\begin{abstract}
Osteoarthritis (OA) is a debilitating joint disease that involves the degeneration of articular cartilage. Although much research has been conducted at the macroscale level, the microscale level is poorly understood. This thesis investigated the micromechanical environment of cartilage cells that is thought to regulate cell metabolic activity.

The cell microenvironment was studied at six distinct locations using a multiscale postprocessing approach. Microscale sub-models were developed with biphasic poroviscoelastic (BPVE) fibril-reinforced materials and tested under axial quasi-static indentation and physiological cyclic sliding.

Elevated principal and shear strains, and decreased fluid pressurization were found with simulated cartilage degeneration. Maximum intracellular compressive (19\%) and shear strain $(15 \%)$ occurred in the superficial zone of OA chondrocytes under sliding loads. Different loading modes resulted in different strains and fluid pressures between cartilage grades that may affect cell metabolism, suggesting that use of non-physiological loads in studies of cartilage could result in erroneous conclusions.
\end{abstract}




\section{Acknowledgements}

First, I would like to thank my parents, Kishwar and Sohail, for always supporting me throughout my graduate studies. Thanks to my two older siblings, Hassan and Faizan, for their motivation and enjoyable company.

I would also like to thank my thesis supervisor, Dr. Andrew Speirs, for his continuous guidance, encouragement, and support throughout my graduate studies. His exceptional research and industrial knowledge have taught me a lot over the years for which I am very grateful.

I must also thank a great friend, and a colleague, Ali Shegaf, who also continuously provided me with great advice and knowledge throughout my studies. I had the pleasure of accompanying him in his office on many occasions for useful and enjoyable conversations. I am also grateful for his collaboration in this thesis, as he provided the macroscale finite element model of both healthy and degenerated cartilage used in Chapters 3 and 4 respectively.

A final thanks to the Department of Mechanical and Aerospace Engineering at Carleton University for providing me with scholarships, and teaching assistantships. 


\section{Table of Contents}

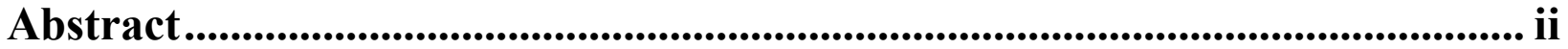

Acknowledgements......................................................................................................... iii

Table of Contents ................................................................................................... iv

List of Figures .................................................................................................... vii

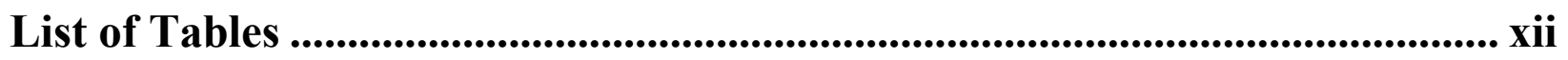

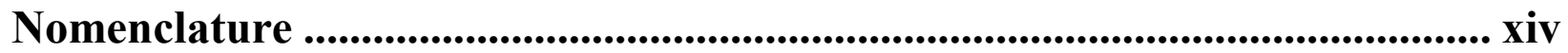

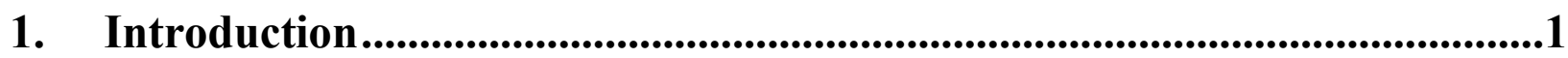

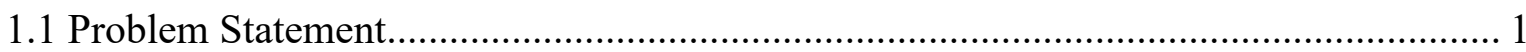

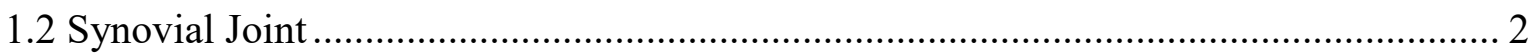

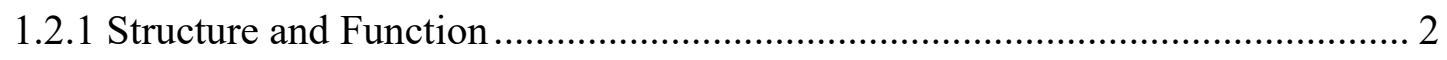

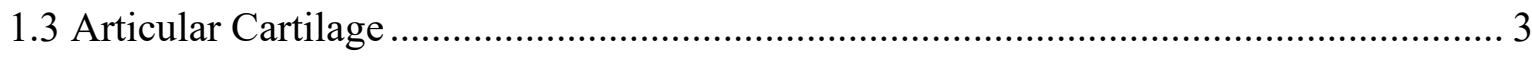

1.3.1 Structure and Composition.......................................................................... 4

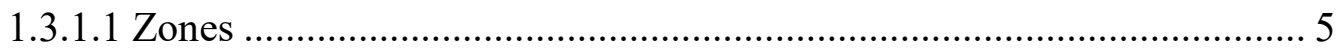

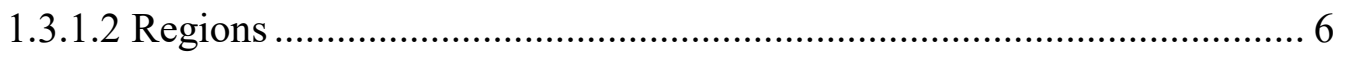

1.3.1.3 Matrix Components ...................................................................... 7

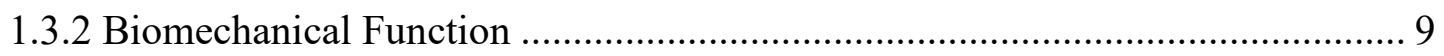

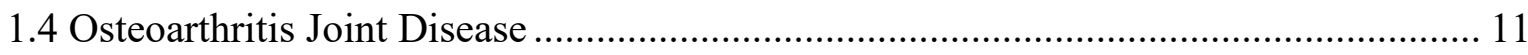

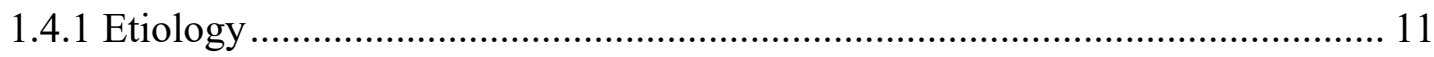

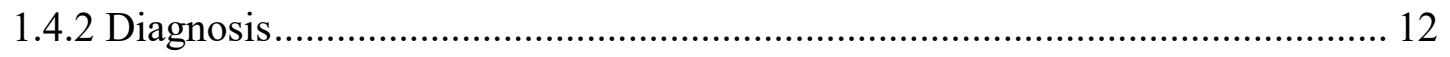

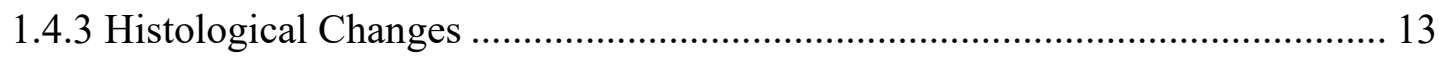

1.4.4 Tissue Metabolism and Gene Expression ...................................................... 15

1.4.4.1 Cell-Matrix Interactions ..................................................................... 16

1.4.4.2 Biological Factors............................................................................ 17

1.4.4.3 Cartilage Mechanobiology ............................................................. 19

1.5 Significance of Multiscale Coupling in Cartilage Biomechanics.................................. 21

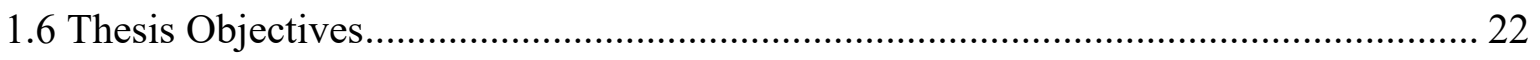

1.7 Thesis Outline

2. Literature Review: Multiscale Finite Element Modelling of Cartilage....24

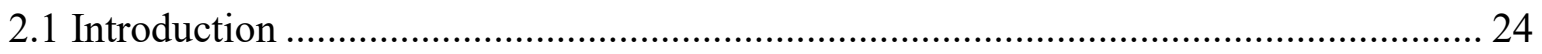




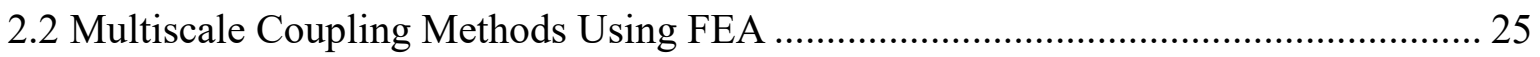

2.3 Theoretical Modelling and Material Properties............................................................ 27

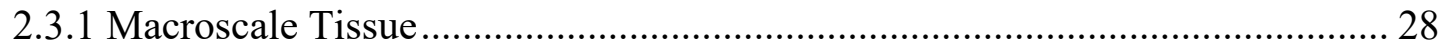

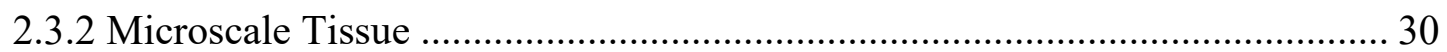

2.4 Typical Loading Configurations........................................................................... 32

3. Biomechanical Analysis of the Microenvironment of Healthy Cartilage. $\mathbf{3 3}$

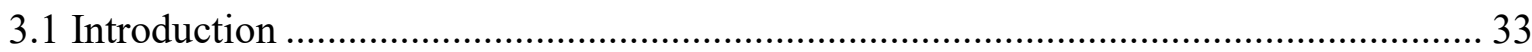

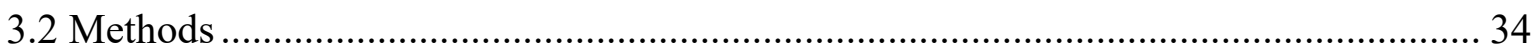

3.2.1 An Overview of the Macroscale Finite Element Model ..................................... 34

3.2.2 Microscale Finite Element Models ................................................................... 38

3.2.2.1 Geometry and Material Properties........................................................ 41

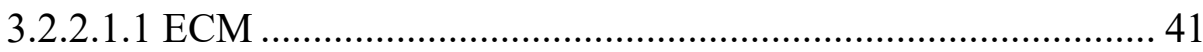

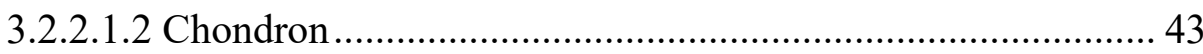

3.2.2.2 Application of Boundary Conditions................................................... 47

3.2.3 Variable Choice and Method of Comparison ................................................... 50

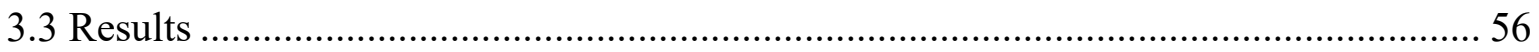

3.3.1 Healthy Cartilage Under Various Loads .......................................................... 56

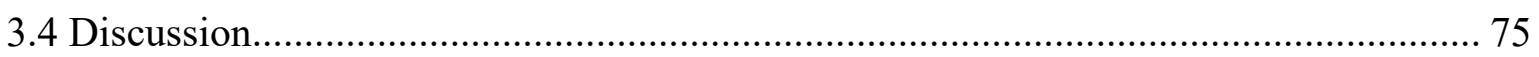

4. Biomechanical Analysis of the Microenvironment of OA Cartilage .........84

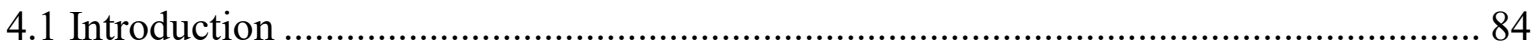

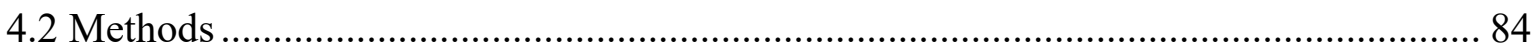

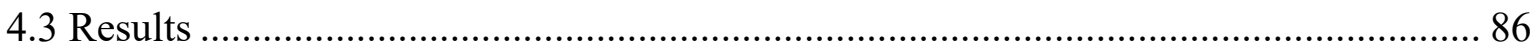

4.3.1 Healthy vs. OA Cartilage Under Cyclic Sliding Indentation............................ 87

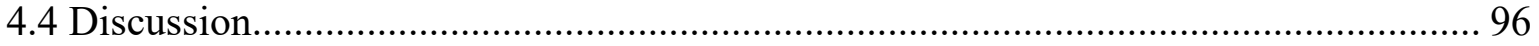

4.4.1 Healthy vs. OA Cartilage Under Cyclic Sliding Indentation .............................. 96

4.4.2 OA Cartilage Under Various Loads................................................................ 98

4.4.3 Differences Between Healthy vs. OA Cartilage Under Various Loads ............ 99

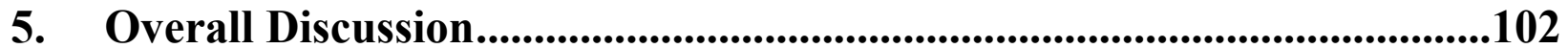

5.1 Cartilage Mechanobiology ..................................................................................... 105

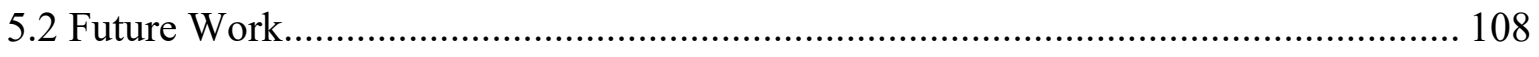

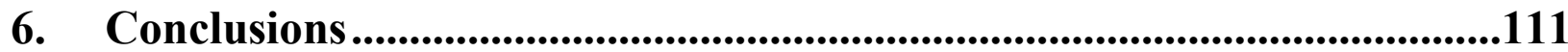

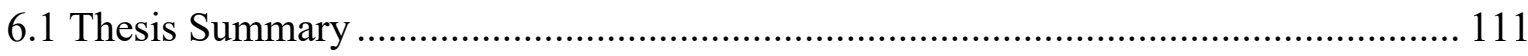


6.2 Thesis Contributions .................................................................................. 113

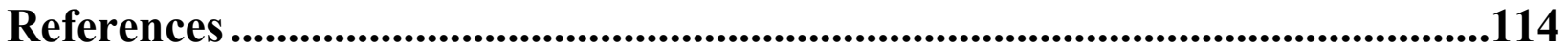

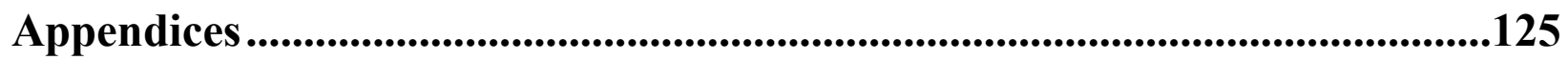

Appendix A : Result Tables for Microscale Models of Healthy Cartilage .................................. 125

Appendix B : Result Tables for Microscale Models of OA Cartilage ........................................... 137

Appendix C : Miscellaneous ........................................................................................... 149

C.1 Mesh Sensitivity Analysis ......................................................................... 149

C.2 Multiscale Homogenization and Deformed Outlines of Selected Models ..... 150

C.3 Additional Contour Plots for Healthy Macroscale Cartilage .......................... 151

C.4 Additional Time Plots for Healthy Cartilage Under Various Loads ............... 153

C.5 Additional Time Plots for OA Cartilage Under Various Loads...................... 157

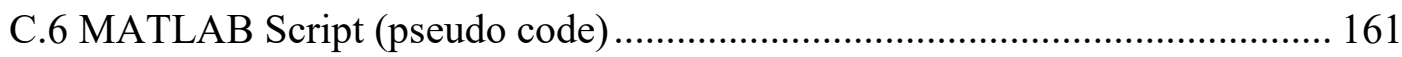

C.7 FEBio2.5 Input Files (pseudo code).............................................................. 165 


\section{List of Figures}

Figure 1.1: Frontal cross-section of a synovial joint showing relevant components................... 3

Figure 1.2: Structure of articular cartilage. Zonal changes in collagen fiber orientation (left), also referred to as the "Benninghoff arcade" [18] provides tensile properties. Zonal changes in chondrocyte shape are shown on the right.

Figure 1.3: X-ray of a human hip joint without (left) and with (right) OA. The joint space (white arrows) narrows due to loss of cartilage tissue in OA. Late stages of OA can cause the underlying subchondral bones to rub against each other resulting in a local increase in bone density (sclerosis). Images courtesy of Dr. Kawan Rakhra, Department of Radiology, Ottawa Hospital.13

Figure 1.4: In-vitro histological changes in PG content of patients with non-OA, moderate OA, and severe OA cartilage. Images courtesy of Andrew Speirs.

Figure 2.1: Multiscale coupling at a synovial joint. A volume element at the cellular level (right) representing the local microenvironment is embedded in a macroscopic finite element mesh at the tissue level (left) representing cartilage at the joint level (not shown). The microscale submodel (right) is typically designed such that a local ECM (red) contains the PCM (yellow), and chondrocyte (blue) inclusions.

Figure 3.1: Fluid flow and displacement boundary conditions imposed on macroscale model. *Denotes contact-dependent fluid flow. At the surface, the fluid is undrained at the cartilageindenter interface and drained at all other locations.

Figure 3.2: Loading protocol employed in multi-scale cartilage models. For both loading protocols, the spherical indenter (red) was initially ( $\mathrm{t}=0$ seconds) positioned at rest $\mathrm{x}=0 \mathrm{~mm}$. At $(\mathrm{t}=2$ seconds), a) the indenter translated downward exerting a 5\% uniaxial compression to the tissue in the negative $\mathrm{z}$ direction. For both static and sliding indentations, this was held constant for 100 seconds. For axial quasi-static indentation, the indenter always remained at a position of $\mathrm{x}=0 \mathrm{~mm}$. For cyclic sliding indentation, (b) at $\mathrm{t}=5$ seconds, the indenter translated to a position of $\mathrm{x}=3 \mathrm{~mm}$. At $\mathrm{t}=8$ seconds, $\mathrm{c}$ ) it returned to the center at $\mathrm{x}=0 \mathrm{~mm}$. At $\mathrm{t}=11$ seconds, it translated to a position of $x=-3 \mathrm{~mm}$. Finally, it translates to the left returning to the center at $x=0 \mathrm{~mm}$. Phases (b-e) represent one cycle for sliding indentation and are repeated for a total of 8 cycles $\approx$ 100 seconds.

Figure 3.3: Microscale sub-model geometry. The local ECM is designed as a cube with dimensions of 100 microns in $\mathrm{x}$, $\mathrm{y}$ (not shown), and $\mathrm{z}$ directions respectively. The depthdependent chondron shape results in a variation in major and minor axes for superficial and deep

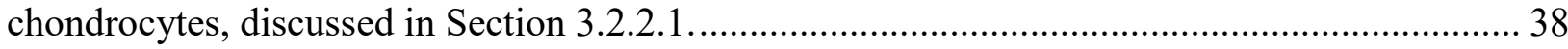

Figure 3.4: Front-view of the loaded portion of the tissue under cyclic sliding indentation, i.e. only 6 of the $20 \mathrm{~mm}$. Locations of the microscale models within the macroscale mesh. The zonal proportions with respect to the tissue thickness $\mathrm{H}$, are shown on the right. The location of the center of the indenter is approximately shown (arrows) above models (A-C) at $\mathrm{x}=0 \mathrm{~mm}$, and (D- 
F) at $\mathrm{x}=2.2 \mathrm{~mm}$ with varying times in the cyclic sliding indentation loading protocol, described in Figure 3.2. Note: Only $6 \mathrm{~mm}$ of the tissue in the $\mathrm{x}$-direction is shown since only $3 \mathrm{~mm}$ of sliding distance is covered by the indenter on both sides of the tissue.

Figure 3.5: Shape and design of chondrocytes implemented in the superficial (A), middle (B), and deep $(\mathrm{C})$ zones. Refer to Table 3.2 for dimensional values.

Figure 3.6: Isoparametric formulation technique used in multiscale cartilage models. The nodes of the original extracted macroscale element of interest (left) in x-y-z coordinate system are denoted with "N\#". The nodes of the corrected unit cube (middle) in the $\Psi-\eta-\zeta$ coordinate system are denoted with "n\#". Linear shape functions were used to determine both geometry and boundary conditions for the remaining nodes of the surface mesh of the microscale model (right). A conventional counter-clockwise node numbering scheme was used as shown. 48

Figure 3.7: Three regional locations within each microscale model were chosen to be studied. The ECM, PCM, and chondrocyte regions were represented by local nodes along the axis of applied load (z-axis).

Figure 3.8: Cycle details for central microscale models in sliding indentation. The total displacement for superficial zone is shown as an example. A total of eight cycles within the simulated time are shown (top) with their respective durations. The exploded view is shown (bottom) for the first complete cycle of sliding motion including the indentation step (red) and beginning of cycle 2 , with arrows indicating the movement of the indenter movement direction in different time periods separated by dotted lines. The position of the indenter relative to the micromodel (yellow) is shown at selected time points. Model size is not shown to scale. Refer to Figure 3.2 for further details on loading protocols.

Figure 3.9: Cycle details for peripheral micromodels in sliding indentation. The total displacement for superficial zone is shown as an example. A total of eight cycles within the simulated time are shown (top) with their respective durations. The exploded view is shown (bottom) for the first complete cycle (red) and beginning of cycle 2, with arrows indicating the indenter movement direction in different time periods separated by dotted lines. The position of the indenter relative to the micromodel (yellow) is shown at selected time points. Model size is not shown to scale. Refer to Figure 3.2 for further details on loading protocols.

Figure 3.10: First principal strain in the microenvironment of healthy cartilage at the superficial, middle, and deep zones of the central (A-C), and peripheral (D-F) locations under cyclic sliding indentation.

Figure 3.11: First principal strain in the microenvironment of healthy cartilage at the superficial, middle, and deep zones of the central (A-C), and peripheral (D-F) locations under axial quasistatic indentation.

Figure 3.12: Maximum first principal strain in the microenvironment of healthy cartilage under different loading modes. 
Figure 3.13: Third principal strain in the microenvironment of healthy cartilage at the superficial, middle, and deep zones of the central (A-C), and peripheral (D-F) locations under cyclic sliding indentation...

Figure 3.14: Third principal strain in the microenvironment of healthy cartilage at the superficial, middle, and deep zones of the central (A-C), and peripheral (D-F) locations under axial quasistatic indentation.

Figure 3.15: Maximum third principal strain in the microenvironment of healthy cartilage under different loading modes.

Figure 3.16: Fluid pressure in the microenvironment of healthy cartilage at the superficial, middle, and deep zones of the central (A-C), and peripheral (D-F) locations under cyclic sliding indentation.

Figure 3.17: Fluid pressure in the microenvironment of healthy cartilage at the superficial, middle, and deep zones of the central (A-C), and peripheral (D-F) locations under axial quasistatic indentation.

Figure 3.18: Maximum fluid pressure in the microenvironment of healthy cartilage under different loading modes.

Figure 3.19: Maximum shear strains in healthy chondrocytes under different loading modes.... 74

Figure 4.1: First principal strain in the microenvironment of OA cartilage at the superficial, middle, and deep zones of the central (A-C), and peripheral (D-F) locations under cyclic sliding indentation.

Figure 4.2: Third principal strain in the microenvironment of OA cartilage at the superficial, middle, and deep zones of the central (A-C), and peripheral (D-F) locations under cyclic sliding indentation.

Figure 4.3: Maximum averaged first principal strain in the microenvironment of healthy vs. OA cartilage under cyclic sliding indentation. 90

Figure 4.4: Maximum averaged third principal strain in the microenvironment of healthy vs. OA cartilage under cyclic sliding indentation.

Figure 4.5: Fluid pressure in the microenvironment of OA cartilage at the superficial, middle, and deep zones of the central (A-C), and peripheral (D-F) locations under cyclic sliding indentation......

Figure 4.6: Maximum averaged fluid pressure in the microenvironment of healthy vs. OA cartilage under cyclic sliding indentation.

Figure 4.7: Maximum shear strains in healthy vs. OA chondrocytes under cyclic sliding indentation. 
Figure 4.8: Absolute differences in the first principal strains of [OA-healthy] cartilage microenvironment under cyclic sliding vs. axial quasi-static indentation.

Figure 4.9: Percent differences in the fluid pressure with cartilage degeneration under cyclic sliding vs. axial quasi-static indentation.

Figure C.1: Influence of mesh refinement in the microscale finite element cartilage models. Mesh refinement beyond 3136 elements resulted in minor differences in axial strain (4\% error).

Figure C.2: A comparison of undeformed and deformed outlines of healthy central microscale models (A-C) at peak ramp load applied to macroscale model at 2 seconds. Contour plots show consistent displacements between both scales.

Figure C.3: Contour plots of $3^{\text {rd }}$ principal strain (top) and stress (bottom) in macroscale cartilage at the end of ramp load ( 2 seconds) and 100 seconds under the novel and physiological cyclic sliding indentation.

Figure C.4: Contour plots of fluid pressurization in macroscale cartilage at the end of ramp load (2 seconds) and 100 seconds under the novel and physiological cyclic sliding indentation...... 152

Figure C.5: Relative volume in the microenvironment of healthy cartilage at the superficial, middle, and deep zones of the central (A-C), and peripheral (D-F) locations under cyclic sliding indentation.

Figure C.6: Relative volume in the microenvironment of healthy cartilage at the superficial, middle, and deep zones of the central (A-C), and peripheral (D-F) locations under axial quasistatic indentation.

Figure C.7: Fluid flux vector plots for selected models subjected to cyclic sliding indentation. Microscale models in the middle zone (B and E) are not shown since they had similar flux patterns to superficial zone (A and D). In all microscale models, the flux is directed along -zdirection during their respective loading phase (a). The flux was generally directed along the $\mathrm{x}-$ axis in the direction opposite to the sliding direction of the indenter (b). During the unloaded phases (c), the flux was generally directed in the $+z$-direction for the microscale models in the superficial zone (A and D) and middle zone (B and E). Whereas, the flux was generally directed along the $\mathrm{x}$-axis for microscale models in the deep zone $(\mathrm{C}$ and $\mathrm{F})$ depending on the relative $\mathrm{x}-$ position of the indenter.

Figure C.8: Fluid flux vector plots for selected models subjected to axial quasi-static indentation. Microscale models in the middle zone (B and E) are not shown since they had similar flux patterns to microscale models A and D. In microscale models A-C, the flux is directed along -zdirection at the end of the peak ramp load ( $\mathrm{t}=2 \mathrm{~seconds})$. At constant load held for 100 seconds, the flux direction changes to $+\mathrm{z}$-direction in microscale models A and B (not shown). However, in microscale model $\mathrm{C}$, the flux direction remains in the -z-direction. In the peripheral locations, the flux vector in microscale models $\mathrm{D}$ and $\mathrm{E}$ (not shown) always remained in the $+\mathrm{z}$-direction over 100 seconds. Whereas, the flux vector in microscale model $\mathrm{F}$ always remained in the $+\mathrm{x}-$ direction over 100 seconds. 156 
Figure C.9: First principal strain in the microenvironment of OA cartilage at the superficial, middle, and deep zones of the central (A-C), and peripheral (D-F) locations under axial quasistatic indentation.

Figure C.10: Third principal strain in the microenvironment of OA cartilage at the superficial, middle, and deep zones of the central (A-C), and peripheral (D-F) locations under axial quasistatic indentation

Figure C.11: Fluid pressure in the microenvironment of OA cartilage at the superficial, middle, and deep zones of the central (A-C), and peripheral (D-F) locations under axial quasi-static indentation

Figure C.12: Relative volume in the microenvironment of OA cartilage at the superficial, middle, and deep zones of the central (A-C), and peripheral (D-F) locations under cyclic sliding indentation 


\section{List of Tables}

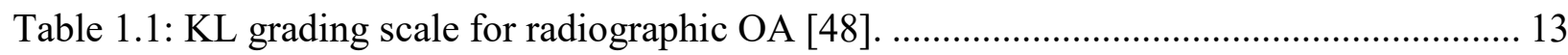

Table 3.1: Microscale model notations used to describe their locations within the macroscale model

Table 3.2: Material properties employed in the ECM, PCM, and chondrocyte regions of biphasic

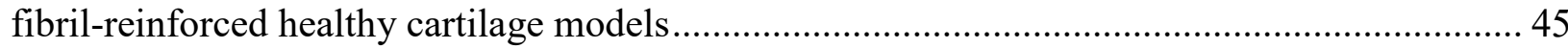

Table 3.3: Dimensions of the chondrocyte shape in each cartilage zone ..................................... 46

Table 3.4: Strain energy densities in selected macro- and micromodels of healthy cartilage under cyclic sliding indentation. 79

Table 4.1: Material properties employed in the ECM, PCM, and chondrocyte regions of biphasic fibril-reinforced OA cartilage microscale models. 86

Table A.1: Summary of selected result variables for the central superficial zone microscale model (A) of healthy tissue subjected to cyclic sliding indentation.

Table A.2: Summary of selected result variables for the central middle zone microscale model (B) of healthy tissue subjected to cyclic sliding indentation.

Table A.3: Summary of selected result variables for the central deep zone microscale model (C) of healthy tissue subjected to cyclic sliding indentation.

Table A.4: Summary of selected result variables for the peripheral superficial zone microscale model (D) of healthy tissue subjected to cyclic sliding indentation.

Table A.5: Summary of selected result variables for the peripheral middle zone microscale model (E) of healthy tissue subjected to cyclic sliding indentation. 129

Table A.6: Summary of selected result variables for the peripheral deep zone microscale model (F) of healthy tissue subjected to cyclic sliding indentation. 130

Table A.7: Summary of selected result variables for the central superficial zone microscale model (A) of healthy tissue subjected to axial quasi-static indentation.

Table A.8: Summary of selected result variables for the central middle zone microscale model (B) of healthy tissue subjected to axial quasi-static indentation.

Table A.9: Summary of selected result variables for the central deep zone microscale model (C)

of healthy tissue subjected to axial quasi-static indentation. 133

Table A.10: Summary of selected result variables for the peripheral superficial zone microscale model (D) of healthy tissue subjected to axial quasi-static indentation. 
Table A.11: Summary of selected result variables for the peripheral middle zone microscale model (E) of healthy tissue subjected to axial quasi-static indentation.

Table A.12: Summary of selected result variables for the peripheral deep zone microscale model (F) of healthy tissue subjected to axial quasi-static indentation. ............................................. 136

Table B.1: Summary of selected result variables for the central superficial zone microscale model (A) of OA tissue subjected to cyclic sliding indentation.

Table B.2: Summary of selected result variables for the central middle zone microscale model

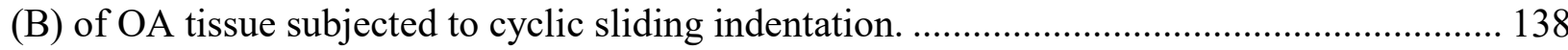

Table B.3: Summary of selected result variables for the central deep zone microscale model (C) of OA tissue subjected to cyclic sliding indentation. 139

Table B.4: Summary of selected result variables for the peripheral superficial zone microscale model (D) of OA tissue subjected to cyclic sliding indentation.

Table B.5: Summary of selected result variables for the peripheral middle zone microscale model (E) of OA tissue subjected to cyclic sliding indentation. 141

Table B.6: Summary of selected result variables for the peripheral deep zone microscale model (F) of OA tissue subjected to cyclic sliding indentation. 142

Table B.7: Summary of selected result variables for the central superficial zone microscale model (A) of OA tissue subjected to axial quasi-static indentation.

Table B.8: Summary of selected result variables for the central middle zone microscale model (B) of OA tissue subjected to axial quasi-static indentation.

Table B.9: Summary of selected result variables for the central deep zone microscale model (C) of OA tissue subjected to axial quasi-static indentation.

Table B.10: Summary of selected result variables for the peripheral superficial zone microscale model (D) of OA tissue subjected to axial quasi-static indentation. 146

Table B.11: Summary of selected result variables for the peripheral middle zone microscale model (E) of OA tissue subjected to axial quasi-static indentation.

Table B.12: Summary of selected result variables for the peripheral deep zone microscale model (F) of OA tissue subjected to axial quasi-static indentation. 148

Table C.1: Influence of mesh refinement in the initial macroscale finite element cartilage model. Mesh refinement resulted in minor differences in the maximum fluid pressure. 149 


\section{Nomenclature}

The following acronyms used in this thesis are listed below:

\begin{tabular}{|cl|}
\hline ADAMTS & A Disintegrin and Metalloproteinase with thrombospondin motifs \\
BMPs & Bone morphogenetic proteins \\
BPVE & Biphasic poroviscoelastic \\
ECM & Extracellular matrix \\
FEA & Finite Element Analysis \\
FGF & Fibroblast growth factors \\
FN-f & Fibronectin fragments \\
GAGs & Glycosaminoglycans \\
HA & Hyaluronic acid \\
HMGB2 & High-mobility group box protein 2 \\
Hz & Hertz \\
IL & Interleukin \\
KL & Kellgren and Lawrence \\
MCP & Monocyte chemotactic protein \\
MIP & Monocyte inflammatory protein \\
MMP & Matrix metalloproteinase \\
MRI & Magnetic resonance imaging \\
NO & Nitric oxide \\
OA & Osteoarthritis \\
PCM & Pericellular matrix \\
PGs & Proteoglycans \\
TGF & Transforming growth factor \\
TNF & Tumor necrosis factor \\
\hline
\end{tabular}


The following symbols used in this thesis are listed below:

\begin{tabular}{|cll|}
\hline Symbol & Description & SI Units \\
$\mathbf{A}$ & Cross-sectional area of the material & $\mathrm{m}^{2}$ \\
$\boldsymbol{\alpha}$ & Material parameter & Dimensionless \\
$\boldsymbol{\beta}$ & Material parameter & Dimensionless \\
$\mathbf{b}$ & Left Cauchy-Green deformation tensor & Dimensionless \\
$\mathbf{E}$ & Young's Modulus & $\mathrm{Pa}$ \\
$\boldsymbol{\varepsilon}$ & Strain & $\mathrm{Dimensionless}$ \\
$\mathbf{H}_{\mathbf{A}}$ & Compressive aggregate modulus & $\mathrm{Pa}$ \\
$\mathbf{H}_{\mathbf{A}+}$ & Tensile aggregate modulus & $\mathrm{Pa}$ \\
$\boldsymbol{\Delta} \mathbf{h}$ & Change in cartilage depth & $\mathrm{m}$ \\
$\mathbf{I}$ & Identity tensor & $\mathrm{Dimensionless}$ \\
$\mathbf{J}$ & Elastic volume ratio & $\mathrm{Dimensionless}$ \\
$\boldsymbol{\kappa}$ & Intrinsic permeability of the medium & $\mathrm{m} / \mathrm{Ns}$ \\
$\boldsymbol{\xi}$ & Fibril stiffness & $\mathrm{Pa}$ \\
$\lambda_{\mathbf{n}}$ & Fiber stretch & $\mathrm{Dimensionless}$ \\
$\mathbf{p}$ & Fluid pressure & $\mathrm{Pa}$ \\
$\boldsymbol{\Delta P}$ & Change in pressure & $\mathrm{Pa}$ \\
$\boldsymbol{\Psi}$ & Strain energy density & $\mathrm{N} / \mathrm{m}^{2}$ \\
$\mathbf{Q}$ & Volumetric flow rate & $\mathrm{m} / \mathrm{s}$ \\
$\boldsymbol{\sigma}_{\mathbf{f}}$ & Fibrillar stress in solid matrix & $\mathrm{Pa}$ \\
$\boldsymbol{\sigma}_{\mathbf{n f}}$ & Non-fibrillar stress in solid matrix & $\mathrm{Pa}$ \\
$\boldsymbol{\sigma}_{\mathbf{s}}$ & Solid stress in poroviscoelastic model & $\mathrm{Pa}$ \\
$\boldsymbol{\sigma}_{\mathbf{t}}$ & Total stress in poroviscoelastic model & $\mathrm{Pa}$ \\
$\mathbf{U}$ & Fluid velocity through the medium & $\mathrm{m} / \mathrm{s}$ \\
$\mathbf{S E D}$ & Strain Energy Density & $\mathrm{J} / \mathrm{m}^{3}$ \\
$\boldsymbol{\mu}$ & Fluid viscosity through the medium & $\mathrm{Ns} / \mathrm{m}^{2}$ \\
$\mathbf{v}$ & Poisson's ratio & $\mathrm{Dimensionless}$ \\
\hline & & \\
\hline
\end{tabular}




\section{Introduction}

\subsection{Problem Statement}

In biomedical engineering, the motivation to study the biomechanics of articular cartilage exists due to pathologies such as OA. OA is a form of arthritis disease which occurs when cartilage tissue, cushioning the ends of long bones, deteriorates due to physical trauma or wear and tear. As a result, patients typically experience joint pain, stiffness, and decreased range of motion as primary symptoms [1]. However, patients may be asymptomatic until medical diagnosis at which time the disease conditions worsen and may require surgical intervention. In Canada, arthritis affects $16.5 \%$ of the population over the age of 15, which is about 4.6 million people as of 2014 [2]. OA is more prevalent in women (19\%) than men (12\%) between the ages of 35-75 [3]. It commonly occurs at the knee, hip, hands, and facet joints [1], which may cause work-related disabilities. Currently $\mathrm{OA}$ is an incurable disease, however, there are several treatment options to enhance the quality of life. At early stages of OA, non-pharmaceutical and pharmaceutical therapies are usually considered. In the case of obesity, physical therapy such as exercise is recommended as it results in weight loss which helps reduce the burden on overloaded joints. Pharmaceutical therapy mainly involves the use of acetaminophen, non-steroidal anti-inflammatory drugs, and invasive medical injections of corticosteroids and hyaluronic acid (HA) which are all effective pain relievers. However, if a patient experiences severe and continuous joint pain with loss of function, then surgical therapy is recommended. Currently, OA is the leading cause of joint replacements [1] which have proven to be tremendously effective with a lifespan of 10-20 years. In Canada, in 2013-14, there were 60,130 and 49,500 patients hospitalized for knee and hip replacements respectively, which 
was an increase of $23 \%$ and $19 \%$ respectively over five years [3]. Arthritis has caused an economic burden on the Canadian healthcare system with $\$ 33$ billion of indirect and direct costs [4] and is predicted to rise with the aging population and obesity rates. Although mechanical overloading and wear are likely major factors of OA [5], the etiology and the process involved in the early stages of degeneration are unknown. In addition to the limited healing capacity of the cartilage itself, the existing treatment options neither terminate nor reverse the process of degeneration. Furthermore, studies in the past have shown that OA concerns more than cartilage degeneration, in fact, it is considered a disease involving multiple joint components [5].

\subsection{Synovial Joint}

Synovial joints are the most common joint type in the body and provide the highest range of motion. Although synovial joints can be further divided into six specific types such as the plane, pivot, hinged, saddle, condyloid, and ball and socket [6], they share a similar anatomical structure.

\subsubsection{Structure and Function}

As shown in Figure 1.1, a synovial joint is composed of bones that articulate within a joint cavity. The walls of this cavity are enclosed by a joint capsule that is continuous with the outer layer of each bone known as the periosteum. Lining the inner and outer walls of the joint capsule are the synovium and fibrous membrane respectively. The synovium and cartilage cells produce lubricin, and HA, which are boundary layer lubricants that make up the molecular components of a viscous non-Newtonian synovial fluid found in the joint cavity [7]. The synovial fluid nourishes the cartilage cells and lubricates the cavity to maintain a nearly frictionless environment. The coefficient of 
friction can be as low as 0.002 , which is approximately one-tenth of the friction generated by skates on ice [6]. Smooth and tough hyaline cartilage covers the ends of articulating bones to provide low friction joint motion. Other accessory components such as tendons, ligaments, muscles, menisci, bursae, and fat pads also play key roles in maintaining the structural integrity of a synovial joint. The overall joint health and function are dependent on the collective effort of the major and accessory components. Degeneration and/or injuries to even one tissue may impact the integrity and function of surrounding components, giving rise to pathological diseases. However, this thesis will focus only on articular cartilage.

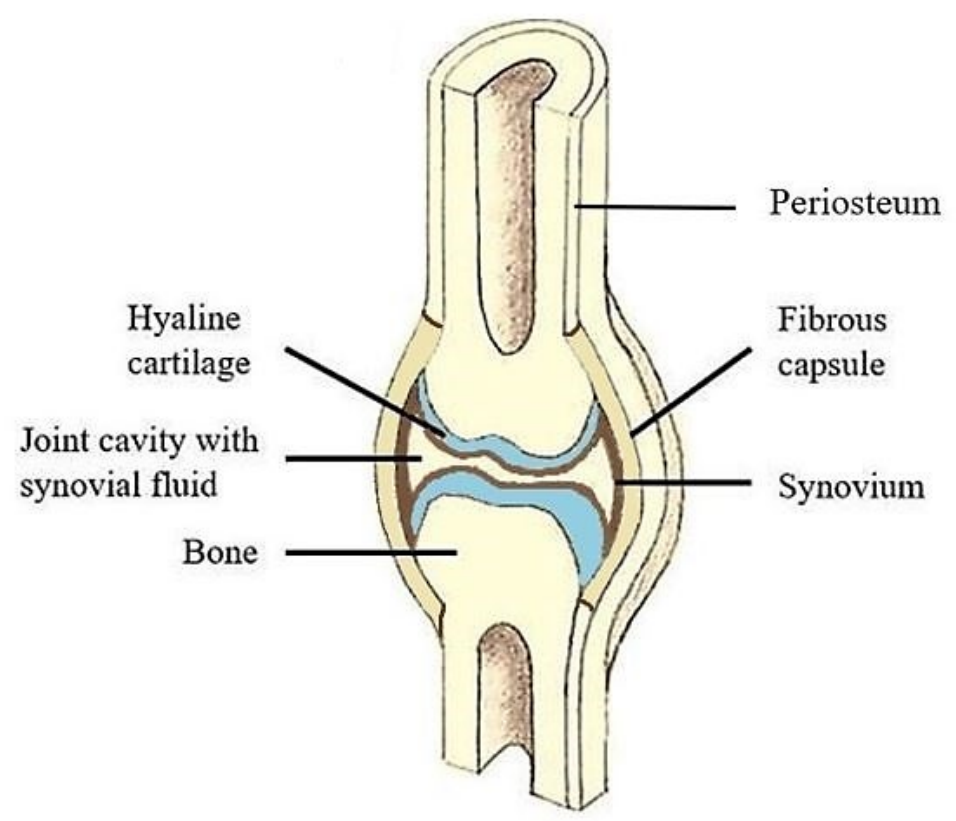

Figure 1.1: Frontal cross-section of a synovial joint showing relevant components.

\subsection{Articular Cartilage}

Cartilage is a connective tissue that is deprived of nerves, blood vessels, and lymphatics. Three types of cartilage are found in the body which vary in composition and function. Elastic cartilage is most pliable and is found in places such as the exterior of the 
ear, and the epiglottis [8]. Fibrocartilage is the toughest and primarily found at the intervertebral disks of the spine, menisci, and at insertion points of tendons and ligaments [8]. Hyaline cartilage is the smoothest and found in areas such as the nose, trachea, rib ends, and at joints [8]. This thesis will focus on hyaline cartilage due to its involvement in OA, a disease of the joint.

\subsubsection{Structure and Composition}

Hyaline articular cartilage is the most abundant type of cartilage with a white glossy appearance and a smooth texture. It covers the ends of long bones with a thickness of 2-4 mm [8]. The tissue consists of a dense extracellular matrix (ECM) composed of mainly solid, liquid, and ionic phases. Fluid is composed of predominantly water that accounts for $65-80 \%$ of the total tissue weight [9], and collagen and proteoglycans (PG's) represent most of the remaining dry weight [9]. The ECM contains other molecules such as lipids, phospholipids, non-collagenous proteins, and glycoproteins distributed in smaller quantities [8]. Chondrocytes are highly specialized cartilage cells also embedded within the ECM, that promote growth, maintenance, and remodeling of the tissue through metabolic processes. The depth of the cartilage tissue can be classified into superficial (tangential), middle (transitional), deep (basal), and calcified zones as shown in Figure 1.2. The cartilage ECM can be further divided as pericellular, territorial, and interterritorial regions [8]. This unique composition and architecture of cartilage contribute to the inhomogeneous biomechanical behavior and function discussed in Section 1.3.2. 


\subsubsection{Zones}

The superficial, middle, and deep zones account for $15 \%, 50 \%$, and $35 \%$ of the tissue thickness respectively [8]. The superficial zone contains the bearing surface and acts as a protective layer to the underlying zones [8]. The chondrocytes in this zone are ellipsoidal in shape and are positioned such that the major axis is tangential to the surface, as seen in Figure 1.2. Similarly, the collagen fibrils (mainly type II and IX) [8] are oriented parallel to the cartilage surface and resist large tensile loading. It also helps resist lateral expansion effectively decreasing the tissue Poisson's ratio which amplifies fluid pressurization. Furthermore, this tangent orientation is believed to inhibit the diffusion of undesirable molecules from the synovial fluid, which contributes to its protective role [8]. Directly beneath the superficial zone is a transitional middle zone that is anatomically the thickest zone in the tissue. Relative to the superficial zone, chondrocytes in the middle zone appear in lower quantities with a spherical shape, and collagen fibers are distributed randomly with thicker diameters [8]. The deep zone lies beneath the middle zone and provides the most resistance to compressive loading due to an abundance of PG content [8] discussed in more detail in Section 1.3.1.3. Unlike the superficial zone, the ellipsoid-shaped chondrocytes in the deep zone are positioned in a columnar orientation such that the major axis is perpendicular to the surface. Likewise, collagen fibers are oriented primarily perpendicular to the cartilage surface and have the thickest diameters [8]. The calcified zone lies immediately below the deep zone and is distinguishable by a tide mark [10]. Chondrocytes in the calcified zone are scarcely distributed and hypertrophic [8], which is believed to be the terminal stage of cell differentiation [11]. Collagen fibers of the deep non-calcified zone continuously cross the tide mark into the calcified zone [12]. These collagen fibers are anchored to the 
underlying high-density subchondral bone securing the cartilage-bone interface, also known as the "osteochondral junction" [12]. This junction exhibits biomechanical and possible biochemical cross-talk which may contribute to the degeneration and maintenance of the overall joint [13]. Subchondral bone contains channels which house blood vessels, and nerves that penetrate as small branches into the calcified zone [12]. The possible biochemical interaction is made possible by the transportation of small particles across the tide mark. A cement line divides these two regions, although no collagen fibers are found crossing this line [14]. Underneath the subchondral bone is a low-density cancellous bone that contains relatively more nerves, and blood vessels than subchondral bone [15] which may play a role in cartilage metabolism and nutrient supply [16]. Cancellous bone consists of pores composed of a network of struts that absorb high impact loads. However, the underlying bones may be subjected to higher contact stress over time due to cartilage degeneration, which could reinforce secondary OA [17].

\subsubsection{Regions}

At the microscopic scale, the ECM of the tissue can be divided into pericellular, territorial, and interterritorial regions [8]. The pericellular matrix (PCM) surrounds the chondrocyte with a thickness of 2.5 microns [18] and is involved in initiating loadinduced signal transduction discussed in Section 1.4.5 [19]. PCM contains mainly biglycan PG's, and type VI collagen fibers [8]. The territorial region is a denser collection of ECM and surrounds the PCM [8]. Collagen fibers appear in mesh-like networks in higher quantities relative to the PCM $[19,20]$. Interterritorial regions fill in the voids in between territorial regions throughout the tissue. The PG content is most 
abundant and collagen fibers form bundles in their respective zone-dependent orientation $[8,21]$.

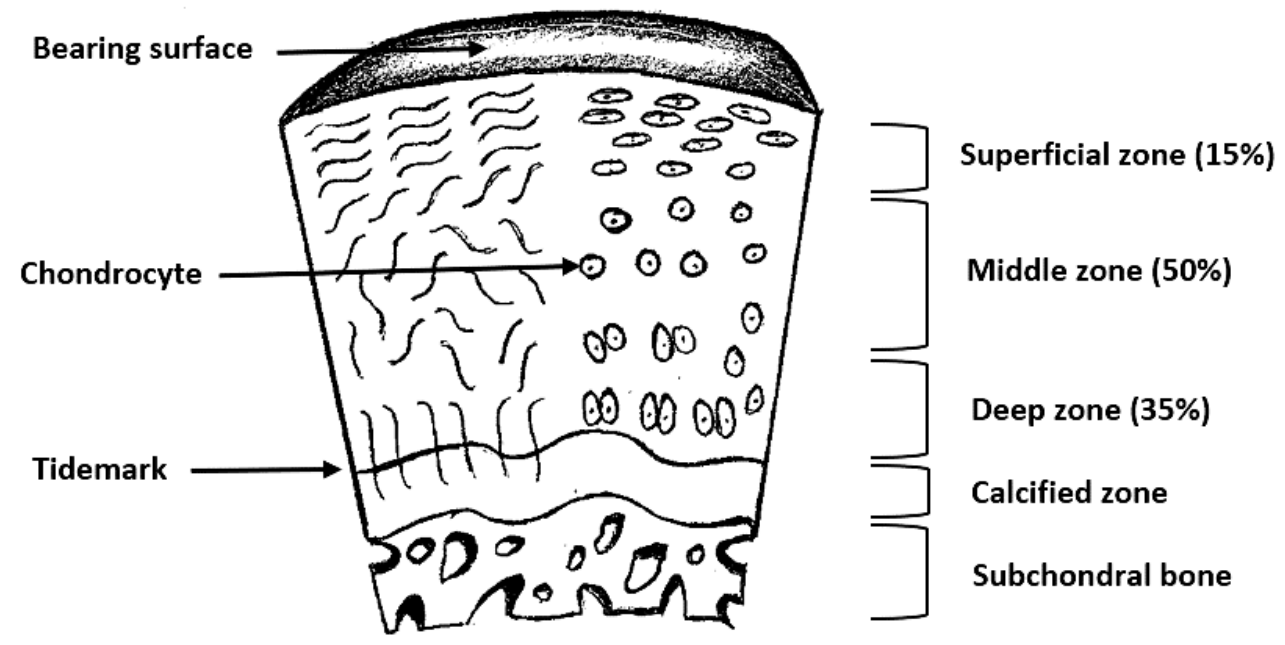

Figure 1.2: Structure of articular cartilage. Zonal changes in collagen fiber orientation (left), also referred to as the "Benninghoff arcade" [18] provides tensile properties. Zonal changes in chondrocyte shape are shown on the right.

\subsubsection{Matrix Components}

As noted earlier, within each region of the ECM, there is the sparse distribution of chondrocytes, proteins, and macromolecules such as collagen and PG's. However, about $80 \%$ of the wet weight of articular cartilage comes from water [21], which is why the structure of the ECM is sometimes referred to as a biological "sponge" [22]. Although most of this water flows within the interterritorial region $[23,24]$, about $30 \%$ is found in the intrafibrillar space with smaller amounts in the intracellular space [8]. The water content decreases from $85 \%$ at the superficial zone to $65 \%$ in the deep zone [8]. Fluid flow is induced by applying pressure gradients across the tissue surface or physiological compression of the tissue $[9,25]$. This flow may be essential for the distribution and transportation of nutrients to chondrocytes [8]. The accumulation of fluid pressure within 
the ECM along with frictional resistance to fluid flow collectively provides the ability for articular cartilage to withstand loads as high as four times the body weight [8], primarily through fluid pressure.

Collagen is a fibrous protein that makes up one-third of the total body protein [22]. It is also the most abundant macromolecule in the ECM of the articular cartilage as it makes up $60 \%$ of the dry weight [8]. Currently, there are twenty-six types of collagen discovered [26], however, only types I, II, IV, V, VI, IX, and XI are found in articular cartilage [8]. The type II collagen accounts for $90-95 \%$ of the collagen found in the ECM [8] with fibers that interweave with the surrounding PG aggregates.

Second to collagen, PG's make up $10-15 \%$ of the wet weight of the ECM [8]. They contain one or more covalently linked glycosaminoglycan (GAG) chains extending from a core protein [8], that resemble a bottle-brush structure. The GAG chains are composed of negatively charged carbohydrate monomers which induce repulsive forces that keep the chains apart [8]. GAG's also have negative surface charges that impede the mobility of bipolar water molecules, thus creating high fluid pressure under load [27]. This hydrophilic property helps tissue rehydration after surface loads are removed.

Aggrecan, the most abundant and largest type of PG's found in articular cartilage is attached to HA through link proteins [28]. This interaction forms larger PG aggregates found in the interfibrillar space which provide cartilage with compressive and osmotic characteristics for the ability to resist large compressive forces [8]. 


\subsubsection{Biomechanical Function}

As noted in earlier sections, articular cartilage serves as a load-bearing tissue and provides a smooth gliding surface at articulation to maintain joint health and function. Ideally, articular cartilage is considered a triphasic tissue composed of solid, liquid, and ionic phases [29]. Although the latter phase contributes to the mechano-electrochemical signal transduction of cartilage, it is beyond the scope of this research. Therefore, this thesis will examine the behaviour of articular cartilage solely as a biphasic medium.

As mentioned in 1.3.1, water is the main ingredient in the fluid phase [8] accompanied by a solid phase which is comprised of a porous and permeable ECM [30, 31] containing mainly the PG's and collagen macromolecules. These phases establish flow-independent and dependent mechanisms that contribute to the poroviscoelastic properties known to withstand high cyclic loads [32]. Since the cartilage tissue is porous with relatively low permeability $\left(10^{-15} \mathrm{~m}^{4} / \mathrm{Ns}\right)[25]$ and velocity, the fluid flow is laminar and can be governed by Darcy's Law. The volumetric flow rate Q is caused by a difference in pressure $\Delta \mathrm{P}$, over a change in depth $\Delta \mathrm{h}$, of a material with cross-sectional area $\mathrm{A}$, and permeability $\kappa$.

$$
Q=-\kappa A \frac{\Delta P}{\Delta h}
$$

As a joint is loaded, the tissue immediately experiences an increase in contact forces and local fluid pressure causing the interstitial fluid to flow out of the loaded area and out of the tissue [33]. The negative sign in equation (1) is required as this considers the fluid flow from high- to low-pressure regions. Small amounts of this fluid seep into the joint cavity, where it may absorb nutrients that are transferred back into cartilage 
upon load removal [34]. The low permeability of the tissue causes a frictional drag between the fluid and ECM which impedes the fluid flow and increases the pressure generated under the loaded area of the surface [31]. The flow is further impeded by the electrostatic forces generated between the negatively charged chains of PGs and the positive ends of the polar water molecules [33]. The carboxyl and sulfate groups of the PG chains provide a fixed charge density to the tissue, resulting in an osmotic pressure, allowing the tissue to resist compression [35]. This osmotic pressure causes swelling of the tissue induced by both the ion concentration within the tissue and chemical expansion of negatively charged PGs is counterbalanced by the collagen network that induces prestresses in the fibrils [36]. The collagen network provides tensile resistance to the tissue which correlates with the zone-dependent orientation of the fibers. It is believed that the intermolecular cross-links of the collagen fibers contribute to their stabilization and ultimate tensile strength [37].

Articular cartilage displays time-dependent creep and stress-relaxation behavior [38]. As cartilage is subjected to constant compressive stress or load, the deformation (creep) increases with time until it gradually reaches an equilibrium state [8]. When fluid gradually flows out due to the pressure gradients generated with loading, tissue consolidation (stiffening) then transfers loads to stress in the ECM solid phase. The equilibrium state is reached when this stress balances the applied surface load [31]. Similarly, as cartilage is subjected to constant strain, the stress reaches a peak value, which then gradually decreases due to tissue relaxation until it reaches equilibrium [31]. With increased levels of strain, the tissue consolidation results in non-uniform changes in the elastic modulus [8]. Under stress-relaxation, this stiffness can be determined for a 
range of applied strain to the tissue. The linear relationship between the equilibrium stress values and applied strain gives the equilibrium modulus [39, 40]. In contrast, creep testing with constant applied stress causes fluid exudation until equilibrium, at which time all the load is carried by the solid matrix. Therefore, the fluid permeability determines the rate of creep whereas the equilibrium displacement determines the compressive stiffness of the solid matrix [41].

\subsection{Osteoarthritis Joint Disease}

\subsubsection{Etiology}

As mentioned in 1.1, OA is classified as a joint disease that may involve multiple components of a synovial joint. OA is more than the wear and tear of joint tissues, it is rather the abnormal remodelling of joint components also caused by biological mediators in the affected joint [5]. The surrounding tissues such as cartilage and bone are influenced by this degenerative process that is known to be irreversible. Primary (idiopathic) OA is a classification used to describe the development of the disease with no identifiable initiating factor [42]. Secondary OA occurs due to a pre-existing condition [42] such as trauma or obesity, which may induce joint over-loading. Biological factors such as nutrition deficiency and congenital or hereditary defects may also trigger the onset of OA symptoms [43]. Other mechanical factors such as muscle weakness, ligament laxity, and meniscal damage may also disrupt the structural integrity $[44,45]$. These can result in joint misalignment and instability, further facilitating the progression of OA [45]. The major pathological features are cartilage degeneration, narrowing of the joint space, the formation of osteophytes (bone spurs), and subchondral bone sclerosis [5]. Additional features are synovium inflammation (synovitis), hypertrophy of the joint capsule, and 
ligament and menisci degeneration [5]. Accessory joint components such as periarticular muscles, nerves, bursae, and fat pads may also contribute to OA symptoms [5].

\subsubsection{Diagnosis}

Plain radiography and magnetic resonance imaging (MRI) are imaging modalities that can be used in the diagnosis of OA [46]. Although these are non-invasive methods, occasionally invasive contrast agents are injected intravenously. Usually, the patient symptoms such as joint stiffness and pain lead to a medical diagnosis which is achieved primarily through plain radiography. As shown in Figure 1.3, high-contrast radiographic images help physicians identify common arthritic features such as subchondral cysts, sclerosis, marginal osteophytes, and narrowing of the joint space [5].

Based on radiographic bone features observed, OA can be categorized to distinguish the level of severity. Kellgren and Lawrence $(\mathrm{KL})$ grading scale, shown in Table 1.1, is the most globally used classification in radiographic OA [47]. Currently, plain radiography is used in clinical practice to confirm OA diagnosis and monitor the progression. Although radiography is relatively cheaper than other modalities, it does not provide direct qualitative or quantitative information about soft tissues such as the cartilage, in which case other modalities may be necessary such as magnetic resonance imaging (MRI) [8]. However, even MRI provides only minimal information about the degeneration process and has limited image resolution. 
Table 1.1: KL grading scale for radiographic OA [48].

\begin{tabular}{cl}
\hline Grade & Description \\
\hline $\mathbf{0}$ & No visible narrowing of joint space or reactive changes \\
$\mathbf{1}$ & Doubtful narrowing of joint space and possible osteophyte lipping \\
$\mathbf{2}$ & Possible narrowing of joint space and definite osteophytes \\
$\mathbf{3}$ & Definite narrowing of joint space, moderate osteophytes, possible deformity \\
& of bone ends and some sclerosis \\
$\mathbf{4}$ & Marked narrowing of joint space, large osteophytes, definite deformity of \\
& bone ends and severe sclerosis \\
\hline
\end{tabular}
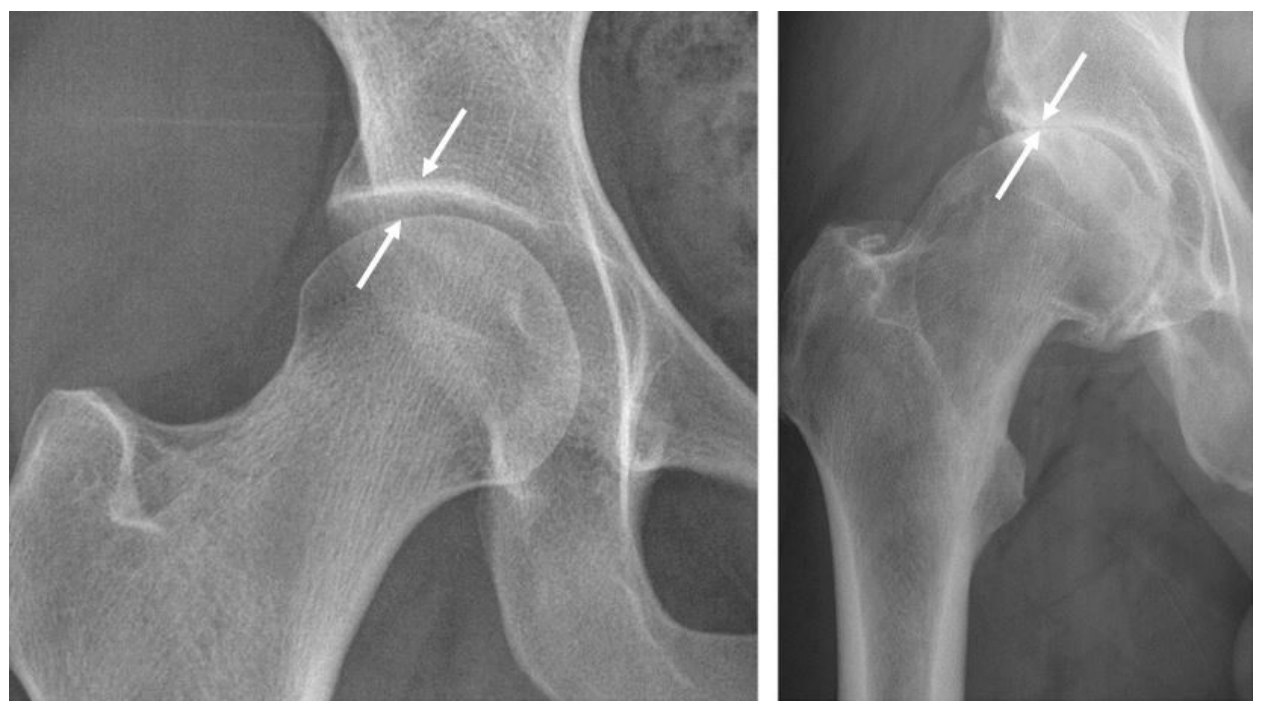

Figure 1.3: X-ray of a human hip joint without (left) and with (right) OA. The joint space (white arrows) narrows due to loss of cartilage tissue in OA. Late stages of OA can cause the underlying subchondral bones to rub against each other resulting in a local increase in bone density (sclerosis). Images courtesy of Dr. Kawan Rakhra, Department of Radiology, Ottawa Hospital.

\subsubsection{Histological Changes}

In-vitro slides of articular cartilage can provide another avenue for investigating histological changes in tissue morphology. Safranin O staining is a method commonly employed to assess pathological changes in PG content. As shown in Figure 1.5, since the intensity of staining is proportional to the PG concentration; different shades of red are 
observed across various grades of OA. The tide mark can be distinguished in these images as a faint blue line. In patients with normal "healthy" cartilage, there is a high intensity of safranin stain indicating an abundance of PG content [49]. In patients with moderate and severe OA, PG content starts to deplete, and the staining intensity reduces significantly. This correlates with an experimentally measured decrease in compressive modulus with the progression of OA [50]. Additionally, PG content is zone-dependent as compressive stiffness increases with depth. This is noticeable in patients with severe OA, where almost all the PG content of the superficial zone has diminished [51, 52]. As a result, the water content is lowest in the superficial zone and increases with depth.
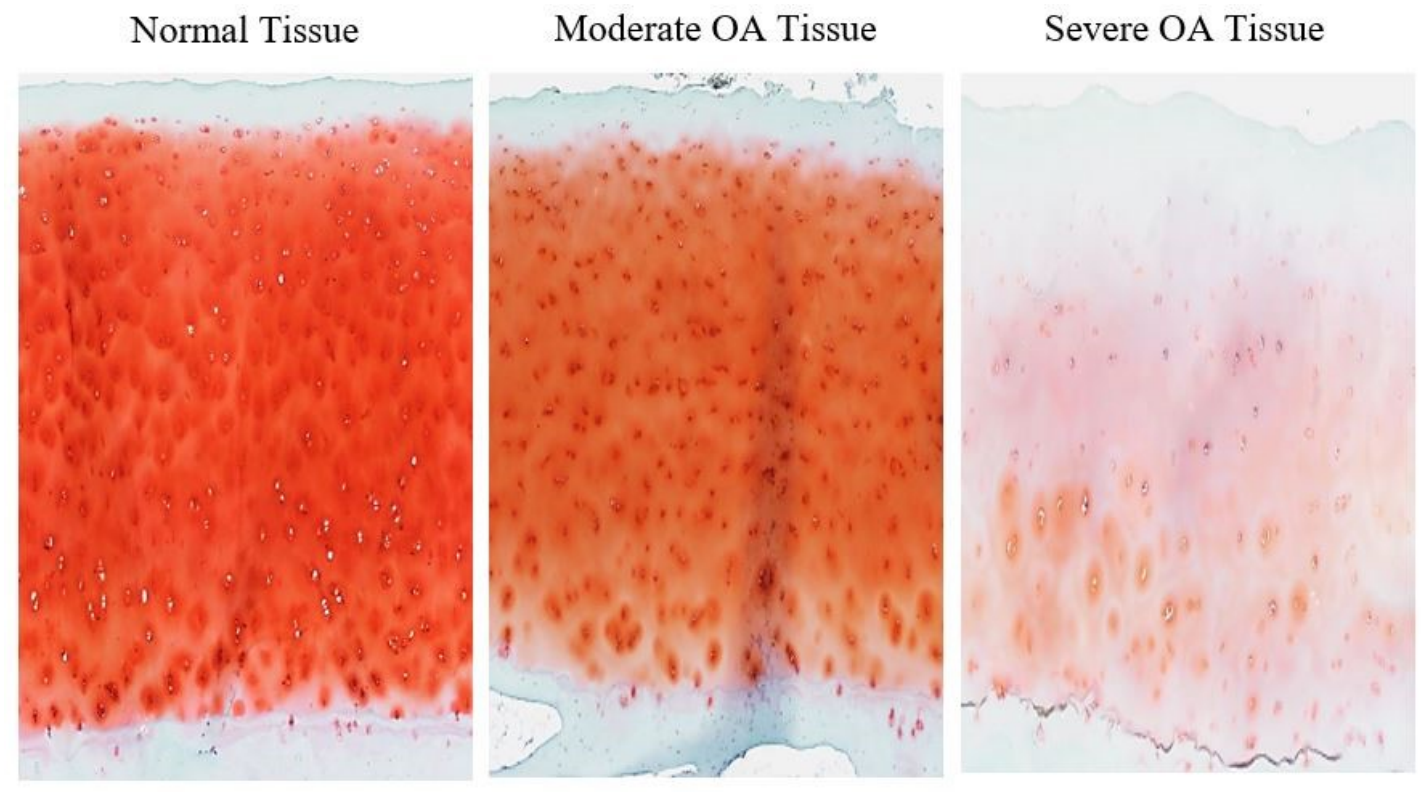

Figure 1.4: In-vitro histological changes in PG content of patients with non-OA, moderate OA, and severe OA cartilage. Images courtesy of Andrew Speirs.

Additionally, the superficial zone contains the highest concentration of oxygen through diffusion from the synovial fluid which decreases in middle and deep zones. Interestingly, chondrocytes in OA tissues tend to group together forming isogenous 
clusters of five to eight cells, mainly found in the deep zone [52]. Although the reasoning is not fully understood, it is believed that the proliferation and migration of chondrocytes may contribute to forming cell clusters [52] driven by tissue metabolism.

\subsubsection{Tissue Metabolism and Gene Expression}

Healthy tissue metabolism is vital for normal cartilage structure and function. Cyclic dynamic loading of the joint allows forces to be distributed across the cartilage tissue and has been shown to maintain healthy tissue metabolism [8], and enhance tissue composition and mechanical properties [53]. Since OA involves multiple components of the synovial joint, cells residing within each tissue respond to changes to their microenvironment that trigger biosynthetic (anabolic) and degradative (catabolic) activities [54]. Similarly, in articular cartilage, chondrocytes synthesize proteins such as the ECM macromolecules and other enzymes that play metabolic roles in regulating matrix turnover [8]. Due to its avascular properties, articular cartilage is considered a postmitotic tissue with low matrix turnover [55]. The supply of nutrients is dependent on diffusion mainly delivered by the surrounding synovial fluid [34]. The 6 nanometer (nm) pore size of the ECM controls the molecule size, configuration, and charge in the diffusion process [9]. During cartilage growth, anabolic processes are highly active as the matrix synthesis occurs more frequently and rapidly by the chondrocytes. As the cartilage tissue matures, the biosynthetic rate decreases significantly and reaches a homeostatic balance [56]. However, soluble factors and mechanical stimuli can disrupt the cartilage metabolism through a series of biological events [56]. The OA degeneration process in the synovial joint is most likely due to abnormal and excessive joint loading. At the microscale, these mechanical stimuli transmitted through the ECM and the fluid are 
converted into electrochemical activity by the chondrocytes, a phenomenon referred to as "mechanotransduction" [55]. This is followed by a cascade of biological activities within the cell that modulates cell metabolism i.e. normal physiological loads induce anabolism to maintain healthy tissue whereas excessive loads induce catabolism in OA. It is believed, at the microscale, this is modulated by the chondrocyte micromechanical environment $[5,57,58,59,60]$, although this is not well understood. Furthermore, the frequency and level of "normal" and "excessive" loads are not well characterized. Many of these biological events are either initiating or feedback amplification factors that promote cartilage destruction irreversibly [5].

\subsubsection{Cell-Matrix Interactions}

The "outside-in" signal transduction from the ECM to chondrocytes is facilitated through mechanoreception, signal transmission, and target activation processes [22]. Mechanoreception is made possible through cell-surface receptors, integrins, and stretchinduced ion channels which are activated through soluble factors, mechanical stimuli, and intercellular communication $[23,57]$. Following cell reception, intracellular signals are transmitted either biochemically or biomechanically [22]. The biochemical pathway involves the activation of second messenger molecules which target and alter specific proteins that further propagate the signal [22]. The biomechanical signal transduction arises from the structural changes in cytoskeleton or mechanoreceptors due to mechanical deformation since both are closely linked at the plasma membrane [22]. Cytoskeletal changes can cause a shift in the organelles, which may impact gene expression [22]. Mechanoreceptors such as stretch-induced ion channels are gated to allow the diffusion of inorganic ions that are regulated through electrochemical gradients [22]. However, 
these channels can open and shut in response to changes in configuration to itself or the attached cytoskeleton due to mechanical deformation, which in turn, could influence other receptors [22]. This poses complications in studying the effect of a specific stimulus on chondrocyte response in-vivo without triggering multiple signals simultaneously. Therefore, many in-vitro studies stimulate the chondrocytes in culture to overcome this challenge.

Major chondrocyte surface receptors found are syndecan-4, toll-like receptors, discoidin domain receptor, frizzled receptors, and cytokine and chemokine receptors [5]. Although these receptors tend to respond to soluble extracellular factors, some may be involved in sensing mechanical factors. Integrins are known to play a major part in mechanotransduction and the pathomechanisms of OA [61]. Integrins are transmembrane heterodimers that bind the cytoskeleton of chondrocytes to extracellular proteins [61]. Integrins can be activated through upstream proteins such as focal adhesion kinase and mitogen-activated protein [61]. Fibronectin is an adhesion protein, often defined as the all-purpose glue for the ECM [22], and plays a crucial role in matrix destruction in the OA degenerative process [61]. As with other mechanoreceptors, mechanical stimuli applied to integrins alters the structure of the attached cytoskeleton, thereby activating adjacent receptors [22], which may influence chondrocyte response.

\subsubsection{Biological Factors}

Chondrocytes and synovium secrete soluble factors in response to changes in tissue metabolism that may promote further anabolic and catabolic effects. The main anabolic factors found in cartilage are bone morphogenetic proteins (BMPs), transforming growth factor (TGF), fibroblast growth factors (FGF), and insulin-like 
growth factor (IGF) $[5,61,62]$. The specific subclasses such as IGF-I, TGF- $\beta-1-2-3$, FGF-2-4-8, and BMP-7 are known to promote matrix synthesis. Interestingly, some of these factors can also play a catabolic role associated with OA.

The main degradative enzymes found in articular cartilage are part of the matrix metalloproteinase (MMP) and A Disintegrin and Metalloproteinase with thrombospondin motifs (ADAMTS) groups [5]. MMP-3 (stromelysin-1) and ADAMTS-4,5 (aggrecanase) degrade aggrecan, whereas, MMP-13 (collagenase) degrade collagen type II fibrous network which is believed to result in an irreversible degeneration stage [5]. The breakdown of cartilage matrix can produce fibronectin fragments (FN-f). FN-f adheres to $\alpha 5 \beta 1$ integrin [61] resulting in a feedback secretion of MMPs, inflammatory cytokines and chemokines, which can further stimulate matrix destruction [5]. Catabolic cytokines such as interleukins (IL) and chemokines along with tumor necrosis factor $\alpha$ (TNF- $\alpha$ ), s100 alarmins and high mobility group box protein 2 (HMGB2) stimulate inflammatory processes mainly acting on articular chondrocytes [63]. This causes an increase in reactive oxygen species and nitric oxide (NO) [5] which disrupt the mitochondrial function in chondrocytes [64] that may lead to cell apoptosis [65]. The increase in NO induced by mechanical stimuli or catabolic cytokines may result in a decrease in autophagy, which is a protective mechanism used by chondrocytes under stress [66]. Additionally, the decrease of HMGB2 expression with increasing age is shown to correlate with loss of superficial chondrocytes [67]. Age may also contribute to the enlargement of chondrocytes known as hypertrophy [5] which marks the terminal stage of differentiation [11]. Hypertrophic chondrocytes are associated with the expression of mainly collagen type X [68], IL-8, and MMP-13 [5]. In early OA, the expression of 
interleukin (IL)-7-15-17-1 $\beta$ cytokines, and s100A-4-8-9-11 alarmins promote synovitis [5]. In later stages of OA, pain levels may be associated with the expression of monocyte inflammatory protein MIP-1 $\beta$ and monocyte chemotactic protein MCP-1 chemokines [5]. However, since cartilage is part of the synovial joint, these mediators may also impact the metabolism of the surrounding tissues directly or indirectly.

\subsubsection{Cartilage Mechanobiology}

In-vitro studies of cartilage enable researchers to understand the effect of an individual stimulus on chondrocyte response as it is difficult to achieve in-vivo. This is usually performed on cartilage explants [58], or biomimetic systems comprising of chondrocytes seeded in 3-D hydrogels [69]. Mechanical in-vitro testing of these samples is usually compressed on a platen device [27], as articular cartilage experiences mainly compressive loading in-vivo. However, shear platen devices also exist [70] in an attempt to apply more physiologically relevant loading to the cartilage surface.

During a lifetime, articular cartilage is subjected to a range of static and dynamic loads with varying frequency and magnitude [71]. It may experience peak stresses as high as $20 \mathrm{MPa}$, and compressive strains of up to $45 \%$ [57]. Cyclic dynamic compression can promote matrix synthesis; however, it is highly dependent on the magnitude and frequency of the applied load. For instance, Kim et al., showed that the synthesis of PG's and other proteins was invariable for load magnitudes less than $5 \%$ at frequencies less than $0.001 \mathrm{Hertz}(\mathrm{Hz})$ in cartilage samples from the knee joint of a calf [59]. However, low magnitude compression with higher frequencies $(0.01-1 \mathrm{~Hz})$ increased PG and protein synthesis by $40 \%$ [59]. The strain rate is also a contributing factor in matrix synthesis. For instance, Kurz et al., showed that a 50\% strain applied at a rate of $0.01 \mathrm{~Hz}$ 
did not have any impact on chondrocyte activity in cartilage explants [72]. However, when applied at a rate of $0.1-1 \mathrm{~Hz}$, it resulted in a substantial decrease in matrix synthesis and increased chondrocyte death [72]. Moreover, the cells that happened to survive this strain rate were unable to respond normally under low magnitude cyclic compression [72].

Similarly, static compression can inhibit matrix synthesis depending on the magnitude and duration of the applied load. It has been shown that $15 \%$ static compression is enough to inhibit matrix synthesis significantly [59]. Removal of load allows tissue recovery, in which case the normal biosynthetic activity is restored [59]. Shear loading devices have shown that dynamic shear strain of $1 \%$ applied at $0.1 \mathrm{~Hz}$ for 24 hours increased protein and PG synthesis by $25 \%$ and $41 \%$ respectively [57]. Studies on isolated human chondrocytes subjected to large shear strains resulted in an increase in GAG synthesis, and expression of IL-6 [73]. In contrast, increased levels of shear stress in chondrocytes produced $\mathrm{NO}[74,75]$, which is a biological marker of $\mathrm{OA}$, as discussed in Section 1.4.5.2. In general, tensile forces experienced by the tissue may activate stretch-induced ion channels allowing diffusion of inorganic ions that may alter protein synthesis, transcription, translation, and gene expression processes [22]. Both compressive and tensile strains have a collective impact on cellular aspect ratio and volume $[76,77,78]$, which may regulate the transport of nutrients and fluid from ECM to chondrocyte.

During compression of articular cartilage, many studies have compared the spatial patterns of matrix synthesis in tissue explants with the patterns of mechanical, electrical, and chemical stimuli based on theoretical models [78]. With this correlation, it was 
predicted that not only deformation but also fluid flow was an important stimulus to consider in the synthesis of ECM by chondrocytes $[57,78]$. As noted in 1.3.2, the fluid pressure contributes to the total load support and reduces stresses acting on the solid matrix. The spatial profile of interstitial fluid flow has been shown to be related to the synthesis of aggrecan, mainly in the middle zone [79]. Additionally, this fluid flow is important for the transport of nutrients across different regions of the tissue [77]. Interestingly, regardless of chondrocyte shape, the cellular response may be a result of fluid shear stresses induced by dynamic flow at the membrane [80] as opposed to normal hydrostatic pressures [81]. These in vitro studies have shown the effect of macroscopic loading of cartilage tissues on chondrocyte metabolism but have not shown the mechanism at the cellular level, i.e., the mechanobiological mechanism. Results from this thesis will build on past studies with novel findings on chondrocyte mechanotransduction and are expected to improve the understanding of the degenerative process and may lead to successful treatments of OA.

\subsection{Significance of Multiscale Coupling in Cartilage Biomechanics}

Past research in the biomechanics of articular cartilage have mainly focused on the joint and tissue scales; however, little attention has been given to the cellular scale. As noted earlier, load transfer from higher scales modulates the biosynthetic response of chondrocytes $[57,72,77]$ by altering their microenvironment. Therefore, linking both macroscopic and microscopic scales can provide a better insight of the in-vivo function and pathology of articular cartilage.

In-vitro experimental studies have shown that although the biomechanical behavior of cartilage is attainable at the macroscale [82], it becomes a challenging task as 
the spatial scale decreases. As a result, researchers have turned to in-silico studies to overcome this challenge. Finite Element Analysis (FEA) is a technique often used to divide a continuous system into discrete sub-elements to study specific details at the point of interest. FEA of cartilage may help bridge the gap between various spatial scales to study the effect of load transfer since the data can be transferred from joint to cellular cartilage models, using simplified but reasonable assumptions. Typically, simple material models are adopted in joint scale models to allow for the implementation of complex geometric and loading scenarios [83]. In contrast, more complex constitutive material models are employed for simple geometric and loading scenarios at the tissue and cellular scales [84]. As shown in Figure 1.2, one could relate tissue and cellular scale models by passing data between scales from a finite element mesh. Various multiscale approaches considered in previous studies on cartilage mechanics will be discussed with choice justification in Chapter 2.

\subsection{Thesis Objectives}

The objective of this thesis is to investigate the biomechanical response of the microenvironment of biphasic fibril-reinforced cartilage models under physiological loading to analyze the effect of load transfer between spatial scales. The analysis is performed at the center and the right periphery of the superficial, middle, and deep layers of cartilage tissues with and without OA (Chapters 3 and 4) respectively. Obtaining deeper insights into chondrocyte mechanobiology may be clinically beneficial in aiding research scientists in the early detection and treatment of OA. 


\subsection{Thesis Outline}

All microscale models were developed in FEBio version 2.5 open-source 3D software with biphasic poroviscoelastic (BPVE) with fibril reinforcement material properties. Each microscale sub-model contains a depth-dependent shape of chondrocytes surrounded by the PCM, and ECM. Central and peripheral spatial locations are modelled from each of three inhomogeneous zones of the tissue described in Section 1.3, resulting in six novel cartilage microscale models. The contributions made in this thesis were presented in two parts listed below;

(1) FEA of healthy cartilage subjected to axial quasi-static indentation and cyclic sliding indentation (Chapter 3).

(2) FEA of OA cartilage subjected to axial quasi-static indentation and cyclic sliding indentation (Chapter 4). 


\section{Literature Review: Multiscale Finite Element Modelling of Cartilage}

\subsection{Introduction}

As discussed in Section 1.5, cartilage biomechanics can be studied at multiple scales to investigate various regions of the joint. Although much research has been conducted at higher spatial scales such as the body, joint, and tissue levels, lower spatial scales such as cellular and molecular levels have only recently gained research focus. Studying the micromechanical environment of chondrocytes is important to gain insight on how the cellular response is modulated. Changes to the chondrocyte microenvironment may cause imbalances in the metabolic processes [5], leading to degenerative changes resulting in OA. However, with present in-vitro technology, it remains challenging to examine the biomechanical behaviour such as strain and fluid flow at the microscale level, which is why in-silico modelling is preferred.

There are various methods commonly employed in joint mechanics to study the effect of load transfer between different spatial scales. At larger body and joint scales, the principles of rigid body statics and dynamics are usually applied to determine joint reaction forces, torques, and motion [85]. At lower tissue and cellular scales, past and recent studies have relied on continuum mechanics methods such as finite element analysis to evaluate parameters of interest [20]. Although the molecular scale is of interest in cartilage nanomechanics, this thesis will focus on coupling tissue and cellular scales since the objective is to gain better insight into chondrocyte mechanobiology influenced by macroscopic load transfer in healthy and diseased tissues. 


\subsection{Multiscale Coupling Methods Using FEA}

There are several in-silico and in-vitro studies on cartilage mechanics that have investigated the relationship between joint loads and chondrocyte response. However, little is known about the chondrocyte micromechanical environment that controls this cellular response. Therefore, this study will explore the significance of chondrocytes surrounded by their immediate PCM, and ECM with varying material properties, boundary constraints, and loading conditions. The important result parameters can be further linked to studies on chondrocyte metabolism which may help clarify how the cellular response is modulated.

In the past, a few multiscale coupling methods have been considered. Modelling the microscale contents within the macroscale model is an approach often used in cancellous bone [86]. However, due to a high finite element count even for smaller volumes, it can become computationally expensive especially with the complex components and geometries of the joint [86]. Ideally, the microscale response determines the overall macroscale mechanical behaviour of cartilage, however modelling in such fashion is challenging. Attempts have been made through computational homogenization, where the solution of a representative microscale sub-model at every integration point in the macroscale finite element mesh determines the material behaviour at the macroscale for desired time steps [87]. The solution obtained at each microscale sub-model could then be applied over a large region to build the entire macroscale model. Although this approach provides a consistent mechanical link between the two scales, it can be computationally expensive since it requires numerical iterations to reproduce the 
macroscale behavior. In complex scenarios, shared memory may need to be used to transfer data rather than the more common distributed memory [77]. Furthermore, since the microscale response is a direct function of the macroscale mechanics, failure of one microscale model convergence may result in complete failure of the macroscopic model [77]. Further complications can arise in modelling OA tissues since there is more variability in composition and mechanical behaviour, and the tissue can be highly heterogeneous as observed in the histology images in Figure 1.4.

A more feasible option is the so-called "autonomous post processing" approach which involves the independent modelling at both scales [77]. At first, a solution is obtained at the macroscale, using a finite element model that represents a tissue as a continuum. Then, a representative volume element is extracted from the macroscale at the desired location and time step. The volume of interest is then modelled separately as a microscale sub-model composed of the appropriate external and internal geometry. Microscale solutions are obtained by applying the boundary conditions from results obtained in the macroscale element to the microscale sub-model. Care must be taken in applying these constraints especially if the elements have different dimensions. Although the autonomous approach forms a weaker link between the scales as compared to the previous two methods, it provides robust multiscale solutions since the simulations are independent of one another [77]. In this case, a more powerful distributed memory is suitable rather than shared memory platforms since no data transfer is necessary between microscale models [77]. For this study, the latter method will be employed in all cartilage models as it is the most feasible option. 


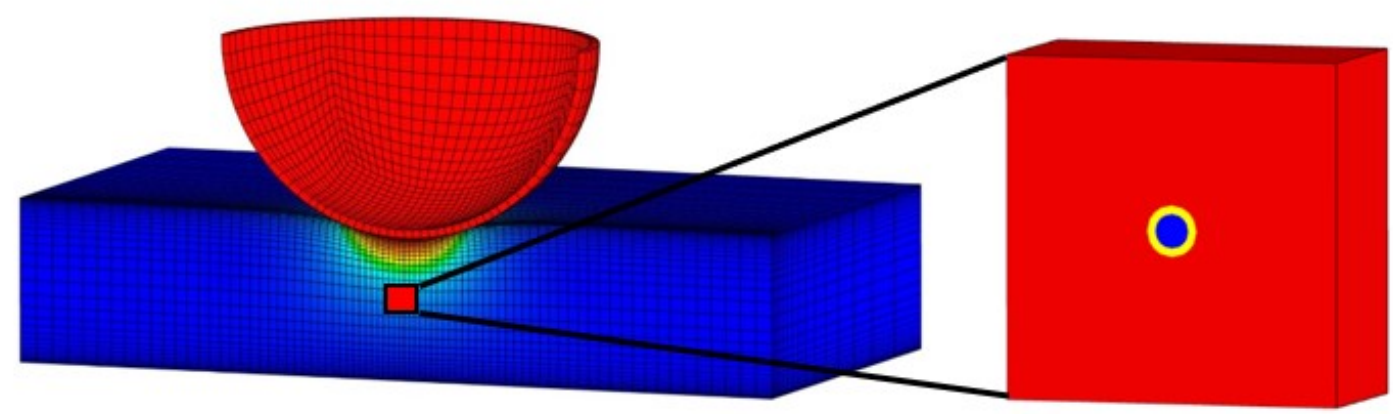

Tissue Level

Cellular Level

Figure 2.1: Multiscale coupling at a synovial joint. A volume element at the cellular level (right) representing the local microenvironment is embedded in a macroscopic finite element mesh at the tissue level (left) representing cartilage at the joint level (not shown). The microscale sub-model (right) is typically designed such that a local ECM (red) contains the PCM (yellow), and chondrocyte (blue) inclusions.

\subsection{Theoretical Modelling and Material Properties}

As mentioned earlier, FEA is a powerful tool in cartilage mechanics as it has the capability of predicting mechanical behaviour at smaller scales of the joint. In preliminary studies, Mow et al., introduced biphasic mixture theories for the macroscopic tissue since cartilage is composed of a $20 \%$ solid phase and an $80 \%$ interstitial fluid phase [25]. Biphasic models and more recently multiphasic models allow for the realistic representation of cartilage behavior, especially under transient or dynamic loads [88]. Multiphasic models consider mixture theories with three or more phases such as cation and anion concentrations within the tissue [88] along with the solid and fluid phases, which can introduce complexities. These phases account for the "Donnan osmotic swelling pressure gradient" caused by the difference in ion concentration of the ECM and the interstitial fluid, chemical expansion stress caused by the repulsion between the negative charges of the PGs, and the chemical potential of the fluid. The electrostatic 
attraction between the negatively charged PG's and the positive ends of the water molecules impedes the fluid flow in addition to frictional drag between the ECM and fluid. For simplicity, this thesis will consider the more commonly used biphasic representation of the tissue comprised of the solid matrix and the interstitial fluid. In this case, the effect of electrostatic forces between PGs and fluid are included in the tissue permeability term of Darcy's Law given in equation (1). To date, no study has quantified the error associated with the exclusion of swelling properties in microscale cartilage. However, a few studies have incorporated a triphasic model both at macro-and microscales and their general influence on the cartilage behaviour. These will be further discussed in Chapter 5.

\subsubsection{Macroscale Tissue}

In earlier studies, the solid matrix of the macroscopic ECM was modelled as a linear elastic tissue [25]. However, since cartilage exhibits time-dependent behaviour, this assumption is not applicable for long cyclic loading conditions, as experienced physiologically. Therefore, modelling the tissue as a BPVE material accounts for this time-dependent physiological response of the solid and fluid mixture [20, 25].

Additionally, numerous studies in the past modelled the solid matrix with homogenous and isotropic properties. However, as described in Chapter 1, cartilage is an anisotropic and inhomogeneous tissue with depth and time-dependent mechanical properties. The inhomogeneous material properties are thought to result from the zone-dependent orientation of the collagen fibers [89]. Donzelli et al. [90], and more recently Federico et al. [91], have demonstrated the importance of transversely isotropic biphasic models by enforcing transverse moduli to the ECM. These models provided a good correlation 
between high stresses and failure points of cartilage compared to isotropic biphasic models and are more sensitive to surface curvature [90]. However, the zone-dependent tangent stiffness and orientation can be alternatively achieved by introducing fibrilreinforcement within BPVE models [92]. These are commonly implemented through constitutive material models or spring elements available within FE software [93]. Furthermore, the structure of the collagen network is formed through individual fibrils that can be classified as the more organized primary fibrils or the less organized secondary fibrils [94]. Korhonen and Herzog have modelled the primary fibrils with the Benninghoff arcade orientation, as shown in Figure 1.2, and the secondary fibrils to be more homogenously distributed throughout the tissue depth [76]. Collagen fibers dominate in tension and are isotropic when unloaded, but as deformation occurs, they become anisotropic. This thesis assumed the collagen fibers of the ECM to have a uniform tangent modulus and spherical fiber distribution throughout the cartilage depth, which will be discussed further in Section 3.2.

In this case, the total effective stress in a BPVE tissue can be predicted by a theoretical model derived from mixture theories [25], as shown in equation (2).

$$
\sigma_{t}=\sigma_{S}-p I
$$

Here, $\sigma_{S}$ is the stress in the solid network, $p$ is the hydrostatic fluid pressure, and $I$ is the identity tensor. The negative sign in equation (2) is present so that the compressive stresses and fluid pressure add up to resist the external forces. The stress of the solid matrix in equation (3), includes the stress in the fibrillar network $\sigma_{f}$, and the non-fibrillar matrix $\sigma_{n f}$, composed of mainly PG's. 


$$
\sigma_{S}=\sigma_{n f}+\sigma_{f}
$$

Substituting equation (3) into (2) results in the total effective stress of a BPVE model with fibril reinforcement, as expressed in equation (4). It should be noted that since the ionic phase is being neglected in this study, the "Donnan osmotic swelling pressure gradient", chemical expansion stress, and the chemical potential of the fluid [36] are excluded from equation (4). The chosen constitutive materials for $\sigma_{n f}$ and $\sigma_{f}$ will be further discussed and justified in the methodology section (Section 3.2) of Chapter 3.

$$
\sigma_{t}=\sigma_{n f}+\sigma_{f}-p I
$$

The solid and fluid phases can be coupled using continuity (equation 5) in one dimension, assumed to be along the z-axis. $U_{z}$ and $\varepsilon_{z}$ are fluid velocity and strain in the z-direction respectively. Continuity can be used to derive a differential equation that can be solved with experimentally determined material parameters and initial and boundary conditions.

$$
\frac{\delta U z}{\delta z}=\frac{\delta \varepsilon z}{\delta t}
$$

\subsubsection{Microscale Tissue}

In the past, the microscale models have been designed such that the ECM encompasses the chondrocyte with a typical diameter of 10 microns $[20,76,95]$ embedded within the PCM with a uniform thickness of 2.5 microns [96]. A single chondrocyte and the PCM (chondron) are commonly placed at the centroid of the desired ECM volume if it is modelled in $3 \mathrm{D}$. The single chondron assumption is based on the scarce population ( $2 \%$ of tissue volume) and sparse distribution of chondrocytes throughout the tissue [20]. Guo and Torzilli have shown that not only zonal but also radial locations of the microscale sub-models within the tissue may also affect the 
biomechanical stresses and strains of chondrocytes [96]. They have also investigated the impact of chondrocyte shape on the solid and fluid microenvironment [96], which assumed an ellipse shape of the superficial and deep zone chondrocytes with a fixed major axis of 15 microns [96]. The major axis was placed in the radial direction in the superficial zone and in the axial direction in the deep zone [96], due to their depthdependent shape illustrated earlier in Figure 1.2. The thickness of the surrounding PCM was kept at 2.5 microns regardless of the shape of chondrocytes [96]. In addition to the PCM, chondrocytes have a bilipid membrane which is usually neglected as these structures have low bending stiffness and are assumed to not contribute significantly to the mechanical behaviour of chondrocytes [20,97]. Like the macroscopic ECM, most studies have treated the chondron as a BPVE material. Bachrach et al., and Gu et al., have described the cytoplasm of the cell to be "gel-like porous permeable fluid saturated meshwork" giving rise to flow-dependent and independent viscoelasticity [20]. Furthermore, the chondron has been commonly modelled with no fibril reinforcement, however, Korhonen and Herzog have shown that chondrocytes are sensitive to volume changes with fibril reinforcement in the PCM [76]. In the case of chondrocytes, it is commonly assumed that the fibers are virtually non-existent. Even though the chondrocytes synthesize collagen fibers along with other macromolecules and proteins, the collagen fibers appear in the pericellular region with increasing density of the network in the interterritorial region, as described in Section 1.3.1.2 [8]. This study will incorporate depth dependent fibril stiffness for the PCM as done by Korhonen et al., [36] but will neglect their presence in the chondrocytes (refer to Section 3.2). 


\subsection{Typical Loading Configurations}

Prior to applying boundary constraints to the microscale model, different loading modes and protocols are applied to the macroscale surface of the tissue. Since cartilage primarily undergoes compression in-vivo, there are compressive loading techniques that have been practiced in the past such as indentation, confined, and unconfined compression. The former experimental method involves a simpler preparation of the apparatus as compared to the other two methods. Cross-validation of BPVE models has been shown to agree well with experimental data for all three loading configurations, further confirming the efficacy of BPVE models [98]. These loading setups provide a global stimulation of the tissue, whereas a novel sliding indentation provides local stimulation $[100,101]$. The sliding indentation is more physiological as it better mimics relative motions of opposing cartilage surfaces under load. This thesis will consider sliding indentation in a cyclic manner along with an axial quasi-static indentation. Although static indentation has been performed in numerous studies, it will be primarily used for comparison and validation purposes. To date, most studies have performed multiscale cartilage modelling in a state of unconfined compression with 2D axisymmetric models $[20,71,76]$ for reducing computational time. This assumption reduces the convergence error and increases the accuracy of the results [101]. However, axisymmetric modelling approaches in cartilage do not allow for the implementation of accurate 3D collagen fiber structure [102] nor do they allow physiological sliding indentation to be implemented since loads are not axisymmetric. Furthermore, since the geometry and boundary conditions acquired from the macroscopic tissue in this thesis were in $3 \mathrm{D}$, the microscale models were also designed in $3 \mathrm{D}$ space. 


\section{Biomechanical Analysis of the Microenvironment of Healthy Cartilage}

\subsection{Introduction}

As discussed in Chapter 2, the most feasible option in coupling both macroscopic and microscopic scales of cartilage tissue is the so-called "autonomous post processing" method. This approach allows the explicit finite element modelling of the tissue at both scales and is not computationally expensive compared to the homogenization method. Since both scales are not a direct function of each other, the failure of one model does not have an influence on the other [77]. When modelling cartilage, the BPVE theoretical model derived from mixture theories is commonly used as it predicts cartilage behaviour more accurately under different loading modes [98] Additionally, fibril reinforcement has also been introduced to represent the accurate depth-dependent orientation and stiffness of collagen fibers within the BPVE model. At a lower microscopic scale, the microenvironment such as the PCM, and chondrocyte (chondron) are also assumed to have BPVE properties [20, 71]. The depth-dependent shape of chondrocytes and their zonal and radial locations within the tissue may contribute to the overall mechanical behaviour $[71,96]$. The presence of collagen fibers is commonly neglected in modelling the chondrocytes but have shown to be significant in the PCM [76].

The goal of this thesis was to analyze how the micromechanical environment of chondrocytes modulates their response in both healthy (Chapter 3) and diseased (Chapter 4) tissues. These will be analyzed at central and radial directions for each of the three zones of cartilage, resulting in six novel BPVE cartilage microscale models. Furthermore, the surface of the macroscale cartilage will be loaded with cyclic sliding indentation, as it 
physiologically mimics joint motion under load and has yet to be studied with the combination of the above parameters. It will also be loaded with a more commonly adopted and less physiological axial quasi-static indentation for comparison purposes.

\subsection{Methods}

\subsubsection{An Overview of the Macroscale Finite Element Model}

The design and analysis for all macro- and microscale models were performed in FEBio version 2.5 open source software that uses an implicit solver with finite deformation. It should be noted that the macroscale model was developed in a separate study, but since it is required for coupling and acquiring input boundary conditions for the microscale model, a brief overview of the macroscale model will be provided.

The macroscale cartilage sample tissue was designed with dimensions of 20, 5 , and $2 \mathrm{~mm}$ in $\mathrm{x}, \mathrm{y}$, and $\mathrm{z}$ directions respectively $[103,104]$. A rigid and impermeable spherical indenter was designed with a diameter of $4.75 \mathrm{~mm}$ with a sliding speed of 1 $\mathrm{mm} / \mathrm{s}$. Experimental testing has shown that velocities of less than $10 \mathrm{~mm} / \mathrm{s}$ for indenter diameters of less than $10 \mathrm{~mm}$ can reproduce physiological conditions such as high fluid load support with low friction $[105,106]$.

The macroscale tissue was discretized with a total of 40500 linear hexahedral elements. This mesh size was chosen based on a mesh sensitivity analysis in Table C.1 in Appendix C, which showed that refining the mesh beyond the indicated number of elements resulted in minor changes in the maximum fluid pressure. As shown in Figure 3.1, the mesh created was biased in the axial and lateral directions to not only minimize 
the computational time with a reduced number of elements but to also capture larger changes in fluid and contact pressures at the cartilage surface.

At the surface, the contact between the indenter and cartilage was established by a "frictionless biphasic contact" implemented in FEBio software using a penalty contact method [107]. The underlying subchondral bone was not included in the macroscale cartilage model, due to its negligible effects on the cartilage surface [108]. However, the effects of the physiological constraints of the impermeable subchondral bone on the cartilage tissue were accounted for by fixing the bottom surface of the cartilage tissue in all directions with no fluid flow. The remaining surfaces were prescribed a free draining condition permitting fluid flow as shown in Figure 3.1.

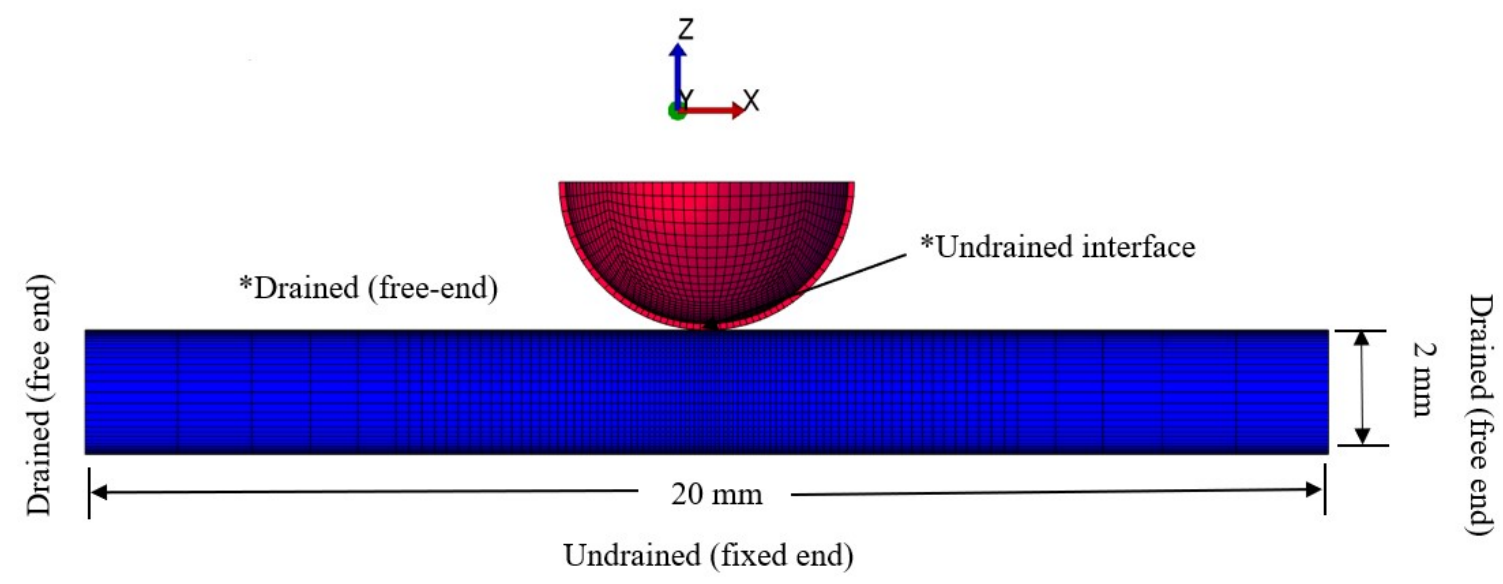

Figure 3.1: Fluid flow and displacement boundary conditions imposed on macroscale model. *Denotes contact-dependent fluid flow. At the surface, the fluid is undrained at the cartilage-indenter interface and drained at all other locations.

Due to the scope of the research, the macroscale model did not account for the depth-dependent structure and composition [109], ionic phases, and the anisotropic hydraulic permeability [110]. The tissue was assumed to be composed of only the ECM containing PGs, collagen fibers, and interstitial water. With these assumptions, the ECM 
was modelled as a biphasic fibril reinforced material with homogenous and isotropic properties at the initial unloaded state. The solid phase consisting of the PGs and collagen macromolecules accounted for the total solid stress $\sigma_{S}$ given in equation 2 .

The PGs were modelled as a hyperelastic neo-Hookean, which results in a nonlinear stress-strain curve at large deformation and is commonly used to model biological tissues [111]. The collagen fibers are modelled as fibers in all directions and were isotropic at their initial unloaded state. Meng at al. showed that these fibers resulted in a similar fluid load support as produced with a realistic fibril orientation [112]. For each material, the constitutive equations describing their behaviour will be presented in the methodology of the microscale model (Section 3.2.2). Furthermore, the fluid phase was modelled using Darcy's Law given in equation 1. Since the deformations in this study were expected to be small, the change in permeability was assumed to be negligible [113]. As a result, the strain-dependent permeability [114] was not considered in this study, as the interstitial hydraulic permeability was assumed to be constant.

Two different loading protocols were considered for indentation at the surface of the macroscale cartilage model (Figure 3.2). In both loading protocols, the initial loading phase consisted of a 5\% axial ramp compression applied linearly over 2 seconds and held constant over 100 seconds. In the novel and more physiological sliding indentation, with constant load, the indenter slides in the positive $\mathrm{x}$-direction to the right side of the tissue at a constant velocity of $1 \mathrm{~mm} / \mathrm{s}$ for a total of 3 seconds. It then slides back to its rest position over another 3 seconds. This protocol is repeated for the left side of the tissue which completes one entire cycle in 12 seconds, excluding the initial ramp load. In the more common and less physiological axial quasi-static indentation, the position of the 
indenter is fixed at the central location with the constant load held for 100 seconds. The load was applied to produce tissue deformation and contact pressure in the physiological range $[115,116]$. Although this is not quite the time at which the tissue reaches a state of equilibrium as found in previous studies $[71,76]$, a simulation time of 100 seconds was deemed sufficient as this was not the main focus of this thesis. As mentioned earlier, the axial quasi-static indentation (creep) loading protocol was performed for comparison purposes since this has been performed in past studies.

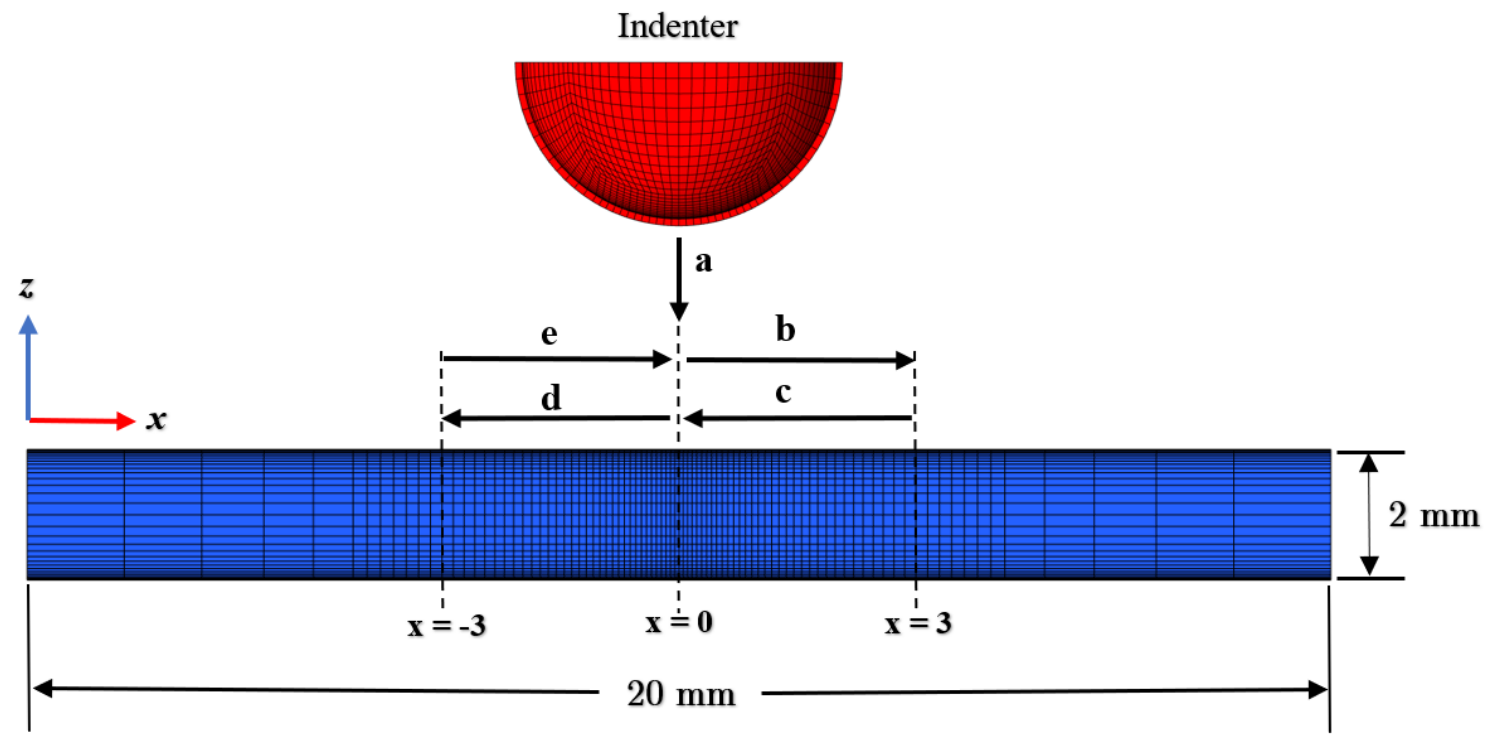

Figure 3.2: Loading protocol employed in multi-scale cartilage models. For both loading protocols, the spherical indenter (red) was initially ( $\mathrm{t}=0$ seconds) positioned at rest $\mathrm{x}=0 \mathrm{~mm}$. At ( $\mathrm{t}=2$ seconds), a) the indenter translated downward exerting a 5\% uniaxial compression to the tissue in the negative $\mathrm{z}$ direction. For both static and sliding indentations, this was held constant for 100 seconds. For axial quasi-static indentation, the indenter always remained at a position of $\mathrm{x}=0$ $\mathrm{mm}$. For cyclic sliding indentation, (b) at $\mathrm{t}=5 \mathrm{~seconds}$, the indenter translated to a position of $\mathrm{x}=3 \mathrm{~mm}$. At $\mathrm{t}=8$ seconds, $\mathrm{c}$ ) it returned to the center at $\mathrm{x}=0 \mathrm{~mm}$. At $\mathrm{t}=$ 11 seconds, it translated to a position of $x=-3 \mathrm{~mm}$. Finally, it translates to the left returning to the center at $\mathrm{x}=0 \mathrm{~mm}$. Phases $(\mathrm{b}-\mathrm{e})$ represent one cycle for sliding indentation and are repeated for a total of 8 cycles $\approx 100$ seconds. 


\subsubsection{Microscale Finite Element Models}

Each microscale model consisted of a chondrocyte cell embedded within the PCM, which were both placed at the centroid of a cubic volume [20], representing the local ECM, as shown in Figure 3.3. This representative cubic volume was designed with dimensions of 100 microns in the $\mathrm{x}, \mathrm{y}$, and $\mathrm{z}$ directions respectively with depth-dependent chondron diameter [96]. Guilak and Mow found that with the presence of a chondrocyte, there was less than $5 \%$ change in the stress and strain fields at a distance of roughly 5 cell radii [20]. Therefore, the microscale ECM boundary is often designed such that it is 50 microns away from the center of the chondrocyte $[20,77]$. The microscale mesh was discretized with 3136 linear hexahedral elements which was chosen based on a mesh sensitivity analysis included in Appendix C.1. It should be noted that large aspect ratios observed in the ECM region were not a huge concern in the design process as the matrix was modelled as a compressible material (Section 3.2.2.1) and no cases of shear locking were reported in FEbio2.5 simulation files.

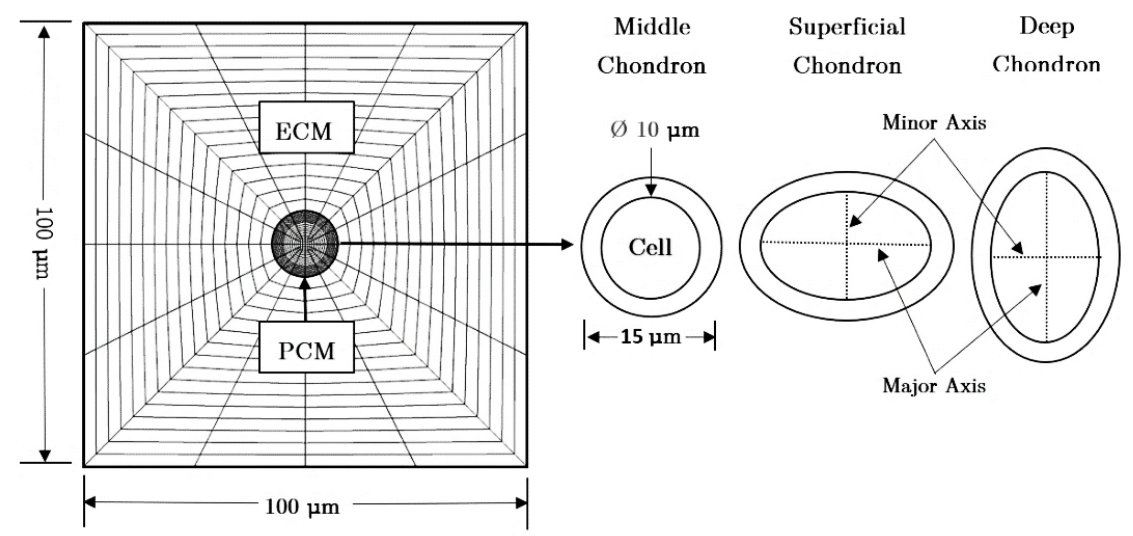

Figure 3.3: Microscale sub-model geometry. The local ECM is designed as a cube with dimensions of 100 microns in $\mathrm{x}, \mathrm{y}$ (not shown), and $\mathrm{z}$ directions respectively. The depth-dependent chondron shape results in a variation in major and minor axes for superficial and deep chondrocytes, discussed in Section 3.2.2.1. 
In this study, both zonal and radial locations of these volume elements were chosen from the macroscale mesh, as shown in Figure 3.4. Two radially located models from the superficial, middle and deep zones were extracted and studied from the macroscale mesh, resulting in six different spatial locations. The two radial models were directed along the positive $\mathrm{x}$-direction with respect to the midline of the macroscale tissue $(\mathrm{x}=0 \mathrm{~mm})$, as shown in Figure 3.4, which was also the center of the indenter at $\mathrm{t}=0$. The two radial models were located at the central $(x=0 \mathrm{~mm})$ and the right periphery $(\mathrm{x}=2.1$ $\mathrm{mm}$ ) of the tissue. These $\mathrm{x}$-coordinates are reported such that the left edge of each micromodel lines up with the center of the indenter. Due to the symmetry of the macroscale tissue, the left peripheral locations of the cartilage tissue were not considered in this thesis. Furthermore, in a previous multiscale coupling study of chondrocytes in a state of unconfined compression, Guo et al. maintained a small distance of $0.1 \mathrm{~mm}$ between the right peripheral models and the edge of the tissue [71]. In the case of sliding indentation adopted in this thesis, the peripheral volume elements were chosen to be $0.8 \mathrm{~mm}$ away from the point at which the center of the indenter reaches its extreme sliding distance of $(\mathrm{x}=3 \mathrm{~mm})$ located at the right of the tissue. In other words, as shown in Figure 3.4, this is approximately located at $(\mathrm{x}=2.2 \mathrm{~mm})$ from the midline of the tissue. The $0.8 \mathrm{~mm}$ distance may seem arbitrary, but it was chosen so that the column of elements in this peripheral region became briefly unloaded as the indenter proceeds to its final $\mathrm{x}$-distance of $3 \mathrm{~mm}$, before sliding back towards them. Additionally, each of the two radial models was extracted from each of the three zones of cartilage based on their respective percent composition of the tissue thickness in the z-direction. As shown on the right of Figure 3.4 , these are $0.15,0.5$, and 0.35 of the tissue thickness $\mathrm{H}$, for superficial, middle, and 
deep zones respectively [8]. The zonal models were extracted such that their location is approximately midway between these values. Explicitly, these are $0.075,0.25$, and 0.175 of $\mathrm{H}$ for superficial, middle, and deep zones, respectively. Table 3.1 summarizes the locations of all six microscale models within the macroscale tissue with a letter notation that will be used for future reference to these models.

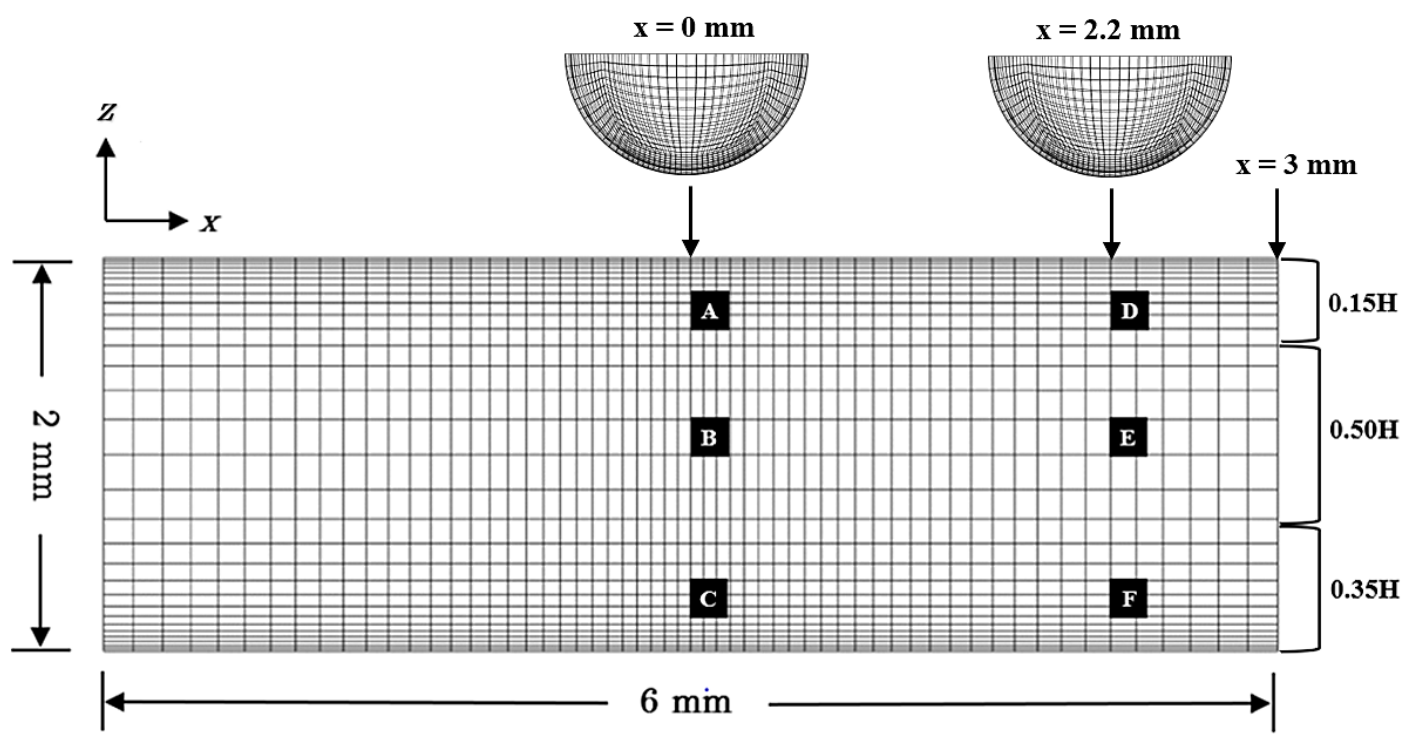

Figure 3.4: Front-view of the loaded portion of the tissue under cyclic sliding indentation, i.e. only 6 of the $20 \mathrm{~mm}$. Locations of the microscale models within the macroscale mesh. The zonal proportions with respect to the tissue thickness $\mathrm{H}$, are shown on the right. The location of the center of the indenter is approximately shown (arrows) above models (A-C) at $\mathrm{x}=0$ $\mathrm{mm}$, and (D-F) at $\mathrm{x}=2.2 \mathrm{~mm}$ with varying times in the cyclic sliding indentation loading protocol, described in Figure 3.2. Note: Only $6 \mathrm{~mm}$ of the tissue in the $\mathrm{x}$-direction is shown since only $3 \mathrm{~mm}$ of sliding distance is covered by the indenter on both sides of the tissue. 
Table 3.1: Microscale model notations used to describe their locations within the macroscale model.

\begin{tabular}{cl}
\hline Model & Descriptive Location \\
\hline A & Center of the superficial zone \\
B & Center of the middle zone \\
C & Center of the deep zone \\
D & Right periphery of the superficial zone \\
E & Right periphery of the middle zone \\
F & Right periphery of the deep zone \\
\hline
\end{tabular}

\subsubsection{Geometry and Material Properties}

\subsection{ECM}

As described earlier, each of the six microscale models was designed such that a 100x100x 100 micron volume of the ECM consisted of the PCM and chondrocyte regions located at the centroid [77]. Each of the six microscale models were discretized with 3136 linear hexahedral (brick) elements, consisting of 3261 nodes. This mesh size was chosen based on a sensitivity analysis, shown in Figure C.1 in Appendix C. It was shown that a mesh refinement beyond 3136 elements resulted in minor differences in the maximum axial strain (4\% error).

Since the ECM is a porous medium with solid and fluid phases and collagen fibers, it was modelled as a biphasic material with fibril reinforcement. The solid phase is modelled as a compressible hyperelastic neo-Hookean material, which represents the PGs. For large deformations, it produces a non-linear stress-strain relationship but reduces to a linear elastic behaviour governed by Hooke's Law for small deformations 
[111]. The constitutive stress equation in the non-fibrillar matrix, namely PGs, is given by;

$$
\sigma_{n f}=\frac{\mu}{J}(b-\boldsymbol{I})+\frac{\lambda}{J} \ln (J) \boldsymbol{I}
$$

In equation $6, J$ is the volume ratio found by taking the determinant of the deformation gradient $(F), b$ is the left Cauchy-Green deformation tensor and can be solved with the deformation gradient $\left(b=F F^{T}\right)$, and $I$ is an identity tensor [111]. The Lamé parameters $\mu$ and $\lambda$ from elasticity theory can be related to the experimentally determined non-fibrillar Young's modulus $(E)$, and Poisson's ratio (v), of the solid matrix.

As mentioned earlier, the fluid flow within the porous non-fibrillar matrix can be described by Darcy's Law given in equation 1. As done in the macroscale model, due to the assumption that deformations are small [113] the hydraulic permeability is assumed to be constant and not strain dependent [114]. The initial permeability is also depthdependent [71], but since the macroscale model assumed a constant permeability within each zone, the ECM region in the microscale models was also kept uniform in all zones for consistency.

Similarly, since the fibril reinforcement in the macroscale model representing the collagen fibers were assumed to have uniform tensile stiffness for all zones, this was also kept consistent for the microscale ECM. This made them unstable on their own [111], therefore, they had to be coupled with a stable compressible material such as the "ground substance", represented by the solid non-fibrillar matrix. The Cauchy stress for this fibril material was modelled as; 


$$
\sigma_{f}=\int_{0}^{2 \pi} \int_{0}^{\pi} H\left(I_{n}-1\right) \sigma_{n} \sin \Phi d \Phi d \theta
$$

where, $H()$ is a unit step function that enforces the fibers to contribute in only tension, and $I_{n}$ is the square of the fiber stretch $\lambda_{n}$ [111]. $I_{n}$ can also be related to the unit vector in its reference configuration which is directed along the fiber direction. This is along $(\theta, \Phi)$ in spherical coordinates [111]. The fiber stress was derived from the fiber strain energy function given by;

$$
\sigma_{n}=\frac{2 I_{n}}{J} \frac{\delta \Psi}{\delta I_{n}} n \otimes n
$$

where the fiber strain energy density is;

$$
\Psi=\frac{\xi}{\alpha}\left(\exp \left[\alpha\left(I_{n}-1\right)^{\beta}\right]-1\right)
$$

where, $\xi(>0)$ is the fiber modulus, $\alpha(\geq 0)$ and $\beta(>2)$ are material parameters [111]. The $\xi$ in the unstretched condition was calculated based on the aggregate modulus in compression $H_{A-}$ and tension $H_{A+}$ [117] given in equation 10.. Since the bundles of collagen fiber with spherical fiber distribution provide the most tensile resistance when parallel to the surface (Figure 1.2), they provide little to no resistance when compressed in one direction, i.e. under axial compression. However, as the tissue expands laterally, the collagen fibers resist that deformation, resulting in anisotropic stiffness.

$$
H_{A+}=H_{A-}+4 \xi
$$

\subsection{Chondron}

The PCM was designed with a uniform thickness of 2.5 microns surrounding the chondrocyte $[71,96]$. Since the PCM was also treated to be a biphasic [20] fibril- 
reinforced [76] material with the same constitutive models, equations 6 and 7 can be substituted in equation 4, resulting in the total effective stress in the PCM. The chondrocyte for each model was embedded within the PCM. As mentioned in Section 2.3.2, the chondrocytes are generally treated as a biphasic medium with no fibril reinforcement [20]. In this case, only equation 6 can be substituted in equation 4 since there is no contribution from the fibrils in the chondrocytes. As assumed for the macroscopic model and the local ECM, the hydraulic permeability is assumed to be constant for both the PCM and chondrocyte. The depth-dependent material properties for the ECM were not considered for consistency with the macroscale model. The material properties used for a biphasic fibril-reinforced macroscale model were $0.83 \mathrm{MPa}, 3.83$ $\left(\mathrm{x} 10^{-15}\right) \mathrm{m}^{4} / \mathrm{Ns}, 0.03$ [118], and 1.2 $\mathrm{MPa}[39]$ for the aggregate modulus, permeability, Poisson's ratio, and fibril stiffness, respectively. In this study, Young's modulus (E) was assumed to be the same as the aggregate (compressive) modulus since the Poisson's ratio used for the macroscale cartilage (ECM) in this study was relatively small and resulted in small lateral deformations.

For the PCM and the chondrocyte, depth-dependent properties were implemented from different experimental studies. For the PCM, this was the uniform value of 0.04 MPa throughout the cartilage depth [71] whereas, for the chondrocytes, these were $4.2 \times 10^{-4}$ and $2.4 \times 10^{-4} \mathrm{MPa}$ for superficial and middle/deep zones respectively as measured by atomic force microscopy [119]. The constant permeability and Poisson's ratio for the PCM and chondrocytes were kept uniform throughout the tissue depth for healthy cartilage as experimentally predicted by the micropipette aspiration technique [120]. The fibril stiffness, $\xi$, was depth-dependent and was calculated based on a 
combination of findings from Korhonen at al. [36], and Tanska et al. [121]. Korhonen at al., provided a non-linear relationship in the depth-dependent tangent moduli for the ECM [36], whereas Tanska et al., predicted the PCM fibril stiffness as 0.1 times the ECM fibril stiffness [121]. Table 3.2 summarizes the material properties used in this study for the ECM, PCM, and chondrocyte regions that were experimentally determined for healthy cartilage.

Table 3.2: Material properties employed in the ECM, PCM, and chondrocyte regions of biphasic fibril-reinforced healthy cartilage models

\begin{tabular}{lllllll}
\hline & $\mathbf{E}(\mathbf{M P a})$ & & \multicolumn{2}{c}{$\boldsymbol{1}(\mathbf{M P a})$} & \multirow{\kappa}{\kappa}{$\left(\frac{\boldsymbol{m}^{\mathbf{4}}}{\boldsymbol{N} * \mathbf{s}}\right) * \mathbf{1 0}^{-15}$} & $\boldsymbol{v}$ \\
\cline { 2 - 5 } & $* \mathrm{SZ}$ & $* \mathrm{MZ} / \mathrm{DZ}$ & $\mathrm{SZ}$ & $\mathrm{MZ} / \mathrm{DZ}$ & All zones & All zones \\
\hline ECM & 0.83 & 0.83 & 1.2 & 1.2 & 3.83 & 0.03 \\
PCM & 0.04 & 0.04 & 0.66 & 0.26 & 0.0419 & 0.04 \\
Cell & $4.2 \times 10^{-4}$ & $2.4 \times 10^{-4}$ & - & - & 5 & 0.38 \\
\hline
\end{tabular}

${ }^{1}$ Fibril stiffness dominates primarily in tension (parallel to bearing surface) and is assumed negligible within the cell. ${ }^{*} \mathrm{SZ}, \mathrm{MZ}$, and $\mathrm{DZ}$ denote superficial, middle, and deep zones respectively.

Both chondrocytes in the superficial and deep zones of the tissue were designed as an ellipsoid shape with a major axis of 15 microns, and a minor axis of 10 microns [96]. The major axis was located radially in the superficial zone and axially in the deep zone [96]. It should be noted that 3D ellipsoid models of the chondrocytes have not been designed to date, therefore no reference material in the literature can be used to validate the geometry in the $y$-axis. In this thesis, the $3 \mathrm{D}$ chondrocytes in the superficial and deep zones were designed such that they are oblate spheroids. This implied that two of the semi-diameters were equal and the third diameter was smaller than the first two with axes $\mathrm{a}, \mathrm{b}$, and c representing the conventional $\mathrm{x}, \mathrm{y}$, and z-axes (Figure 3.5). For superficial 
chondrocytes, radii a and b were fixed at 7.5 microns, whereas $\mathrm{c}$ was 5 microns [96].

Similarly, deep chondrocytes were designed like the superficial chondrocytes but rotated about the b-axis. The middle chondrocytes had an equal radius of 5 microns in all $\mathrm{a}, \mathrm{b}$, and $\mathrm{c}$ directions, reducing it to the normal sphere. Table 3.3 provides a summary of the depth-dependent shape dimensions of the chondrocyte.

A
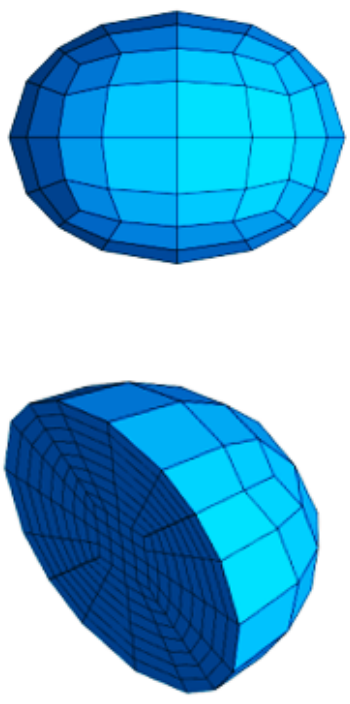

B
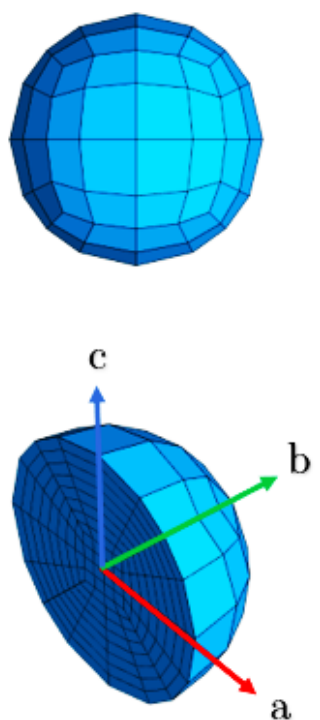

$\mathrm{C}$
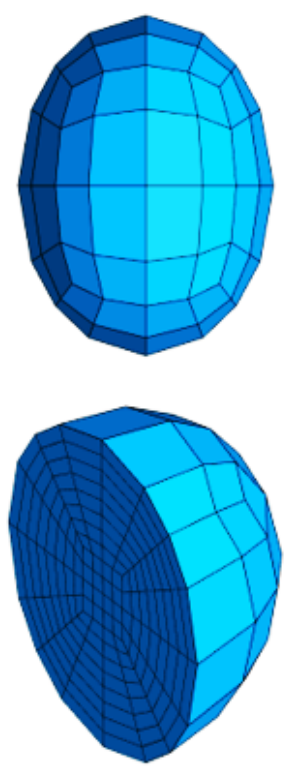

Figure 3.5: Shape and design of chondrocytes implemented in the superficial (A), middle (B), and deep (C) zones. Refer to Table 3.2 for dimensional values.

Table 3.3: Dimensions of the chondrocyte shape in each cartilage zone

\begin{tabular}{cccc}
\hline Zonal Chondrocytes & $\mathbf{a}(\boldsymbol{\mu m})$ & $\mathbf{b}(\boldsymbol{\mu m})$ & $\mathbf{c}(\boldsymbol{\mu m})$ \\
\hline Superficial & 7.5 & 7.5 & 5 \\
Middle & 5 & 5 & 5 \\
Deep & 5 & 7.5 & 7.5 \\
\hline
\end{tabular}




\subsubsection{Application of Boundary Conditions}

As discussed in Chapter 2, the autonomous post-processing approach can be used to couple the macro- and microscale models using finite element methods. The previous section covered the geometry, discretization of the continuum, and the material properties for all microscale models. This section will discuss the method employed in applying the boundary conditions from the macro- to microscale to investigate the effect of load transfer on the chondrocyte response.

In this study, the boundary conditions applied to the microscale models can be linked to the macroscale through the finite element mesh. The displacements in the $\mathrm{x}, \mathrm{y}$, and $\mathrm{z}$ directions and effective fluid pressure at each of the 8 nodes of the chosen macroscale hexahedral elements were extracted and applied to all the surface nodes of the microscale mesh $[20,71,77,96]$. However, since the macroscale model used in this study consisted of a biased mesh, the macroscale hexahedral elements were not all the same sizes and did not match the designed geometry of the microscale models. Due to this, there were cases where more than one macroscale element was required for interpolation. However, in any case, the corrected natural coordinates were determined in the $(\Psi, \eta, \zeta)$ coordinate system with respect to the macroscale coordinate system $(\mathrm{x}, \mathrm{y}, \mathrm{z})$ using an isoparametric formulation technique. Prior to this calculation, a numbering scheme was established for each of the 8 nodes of the original element $(\mathrm{N})$ and corrected element (n), shown in Figure 3.6. Node 1 of both the original and corrected elements was set equal to each other and used as a reference node to determine the natural coordinates. Since the natural coordinates were dependent on the position vectors manually extracted 
from the chosen macroscale elements, a general script was written in MATLAB software (Appendix C.6).
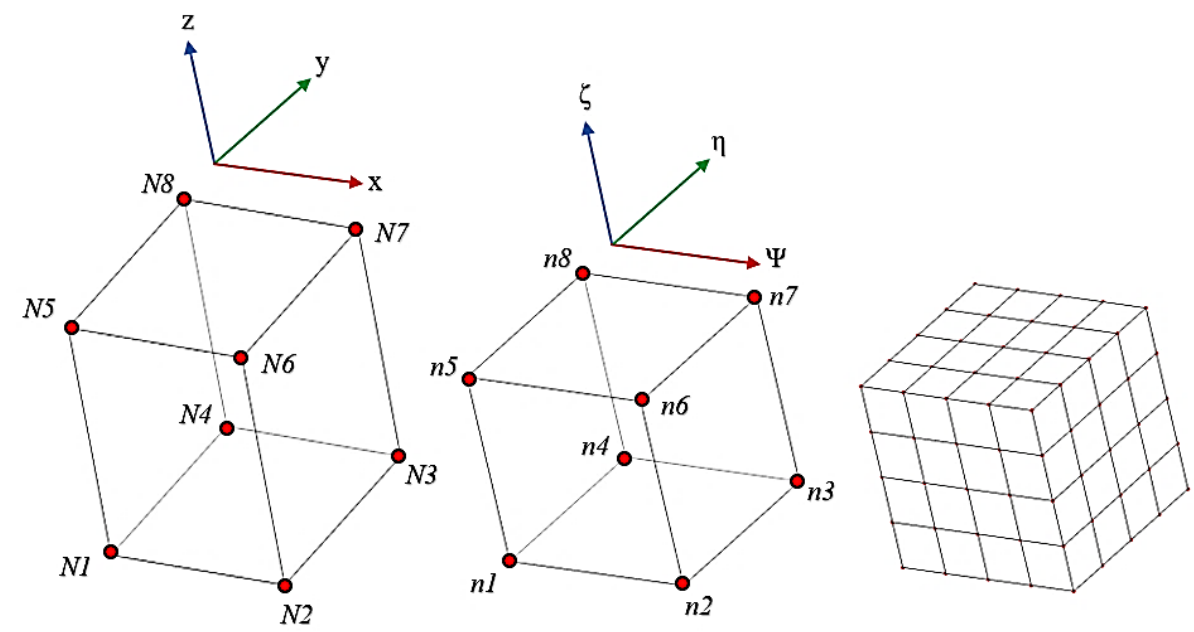

Figure 3.6: Isoparametric formulation technique used in multiscale cartilage models. The nodes of the original extracted macroscale element of interest (left) in $x-y-z$ coordinate system are denoted with "N\#". The nodes of the corrected unit cube (middle) in the $\Psi-\eta-\zeta$ coordinate system are denoted with " $n \#$ ". Linear shape functions were used to determine both geometry and boundary conditions for the remaining nodes of the surface mesh of the microscale model (right). A conventional counter-clockwise node numbering scheme was used as shown.

Once the natural coordinates of each microscale element were calculated with respect to the reference node in the 3 axes, linear shape functions i.e. the slope of the position vectors were used to calculate the $x-y-z$ displacements and fluid pressure boundary conditions. These boundary conditions had to be manually imported into MATLAB prior to performing the linear interpolation. Since the reference node $(N 1=n 1)$ were set equal to each other, they also shared the same boundary conditions. This provided an advantage in the linear interpolation as it resulted in four unknowns $(\mathrm{x}-\mathrm{y}-\mathrm{Z}$ displacements and fluid pressure) at each node and four interpolating functions, as shown in equations 11-14. These are a generalized form of the shape functions as the boundary 
conditions determined in this thesis were both location and time-dependent and were accounted for using "for-loops" in MATLAB software (Appendix C.6).

$$
\begin{aligned}
& x_{j}(t)=\left[\frac{X_{j}(t)-X_{i}(t)}{V c_{J}-V c_{i}} *\left(v c_{j}-v c_{i}\right)\right]+x i(t) \\
& y_{j}(t)=\left[\frac{Y_{j}(t)-Y_{i}(t)}{V c_{J}-V c_{i}} *\left(v c_{j}-v c_{i}\right)\right]+y i(t) \\
& z_{j}(t)=\left[\frac{Z_{j}(t)-Z_{i}(t)}{V c_{J}-V c_{i}} *\left(v c_{j}-v c_{i}\right)\right]+z i(t) \\
& f_{j}(t)=\left[\frac{F_{j}(t)-F_{i}(t)}{V c_{J}-V c_{i}} *\left(v c_{j}-v c_{i}\right)\right]+f i(t)
\end{aligned}
$$

In equations 11-14, the $x-y-z$ displacements and fluid pressures acquired from the macroscale volume elements are denoted by $\mathrm{X}, \mathrm{Y}, \mathrm{Z}$, and F, whereas the calculated corrected boundary conditions are denoted by $\mathrm{x}, \mathrm{y}, \mathrm{z}$, and $\mathrm{f}$. These boundary conditions are not only dependent on their location within the tissue but also are a function of time, and therefore, are accounted for with (t) notation. The corresponding components of the position vectors of the original macroscale and corrected microscale elements are denoted by $V c$ and $v c$, respectively, and depend on the axis of linear interpolation. The letters $\mathrm{i}$ and $\mathrm{j}$ denote the two node numbers along these axes. Once the boundary conditions were calculated for each of the corner nodes of the corrected microscale element, the same shape functions were used to interpolate the boundary conditions of the remaining nodes on the microscale surface mesh, ensuring that overlapping nodes were not accounted for more than once. Since each of the 98 nodes on the microscale surface mesh consisted of 4 time-dependent boundary conditions, a total of 392 load-curves were prepared in a (.txt) 
file for each cartilage zone, location, grade, and loading conditions for a total of 24 distinct models. This was achieved through a MATLAB script (Appendix C.6) in a format compatible with FEbio input files (Appendix C.7) for the purpose of avoiding manual and time-consuming application of nodal boundary conditions.

\subsubsection{Variable Choice and Method of Comparison}

When modelling cartilage at the microscale, it is important to distinguish which result variables are defined as "influential". As discussed in Chapter 1, static compression can inhibit matrix synthesis depending on the magnitude and duration of the applied load [22]. In contrast, cyclic dynamic compression can promote matrix synthesis depending on the magnitude and frequency of the applied load [22]. With these types of loading protocols, many studies have compared the spatial patterns of matrix synthesis in tissue explants with the patterns of mechanical, electrical, and chemical stimuli based on theoretical models [78]. Collectively, it was concluded that not only compressive and tensile deformation but also the fluid flow are all important stimuli to consider in the synthesis of ECM by chondrocytes $[57,78]$. As described in 1.3.2, the fluid pressure contributes to the total load support and reduces stresses acting on the solid matrix. The spatial profile of interstitial fluid flow has shown to be related to the synthesis of aggrecan, mainly in the middle zone [79]. Additionally, this fluid flow is important for the transport of nutrients across different regions of the tissue [77].

Studies on isolated human chondrocytes subjected to large shear strains resulted in an increase in GAG synthesis, and expression of IL-6 [73]. In contrast, increased levels of shear stress in chondrocytes produce $\mathrm{NO}[74,75]$, which is a biological marker of OA, as discussed in Section 1.4.5.2. In general, chondrocytes subjected to tensile loading may 
activate stretch-induced ion channels allowing diffusion of inorganic ions that may alter protein synthesis, transcription, translation, and gene expression processes [22]. Both compressive and tensile strains have a collective impact on cellular aspect ratio and volume $[76,77,78]$, which may regulate the transport of nutrients and fluid from ECM to chondrocyte. In light of these facts, the output variables chosen for investigation in this thesis were the $1^{\text {st }}$ and $3^{\text {rd }}$ principal strains, fluid pressure and flux, and relative volume for the ECM, PCM, and chondrocytes. Additionally, the effect of shear stresses and strains were also studied in the chondrocytes only. The results section will present the analysis of superficial, middle, and deep zone microscale models at the central and peripheral locations of the healthy (Chapter 3) and OA (Chapter 4) cartilage. The ECM node was selected at the edge of the microscale model; the PCM and chondrocyte nodes are located at the middle of the respective regions. For simplicity, the results were extracted from three spatial nodes representing these regions in the axis of applied compression, as shown in Figure 3.7.

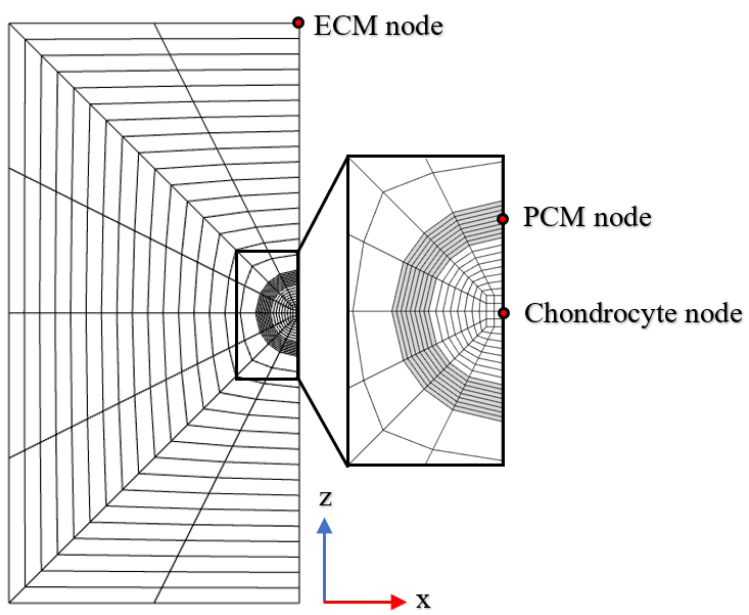

Figure 3.7: Three regional locations within each microscale model were chosen to be studied. The ECM, PCM, and chondrocyte regions were represented by local nodes along the axis of applied load (z-axis). 
As mentioned above, the variables chosen to investigate the micromechanical environment of cartilage in this study were the $1^{\text {st }}$ and $3^{\text {rd }}$ principal strains, relative volume, fluid pressure, and fluid flux. The maximum solid shear stresses and strains were also reported for the chondrocyte, but along with fluid flux were not graphically shown. The tabulated numerical values for all output variables studied in this thesis for healthy cartilage under both loading conditions are summarized in Appendix A.

In this study, all microscale models were subjected to two different loading modes, namely, the cyclic sliding indentation, and creep. The latter will be referred to as "axial quasi-static indentation" and is a typical loading mode used in biomechanical studies of cartilage $[92,122]$. Both of their loading protocols are described in Figure 3.2. For the former loading mode, the sliding motion is cyclic and thus the maxima and minima are averaged with time in each cycle studied in this thesis, i.e. summary tables of sliding indentation in Appendix A and B. Since the simulations were run for 100 seconds, only the averaged peaks and valleys are reported for cycles 1 and 8 for sliding indentation. Therefore, the term "averaged" used in this thesis will imply the abovementioned method for the cycle of interest. Whereas for axial quasi-static indentation, the peaks at the end of the ramp load and at 100 seconds are reported and analyzed.

Since both central and peripheral microscale models were a set distance apart, there was a phase lag between the start and end of their loaded and unloaded regions, but each cycle for both locations had the same duration of 12 seconds due to the constant velocity of the indenter. The number of cycles and their corresponding time durations for the central and peripheral micromodels is shown in Figures 3.8 and 3.9, respectively. As observed for both locations under sliding indentation, the total displacement shows a 
slow shift over time in the mean values. This was most likely due to tissue consolidation that occurs over time as more fluid leaves the tissue than enters upon load removal. It is analogous to creep under a non-zero time-averaged load. 

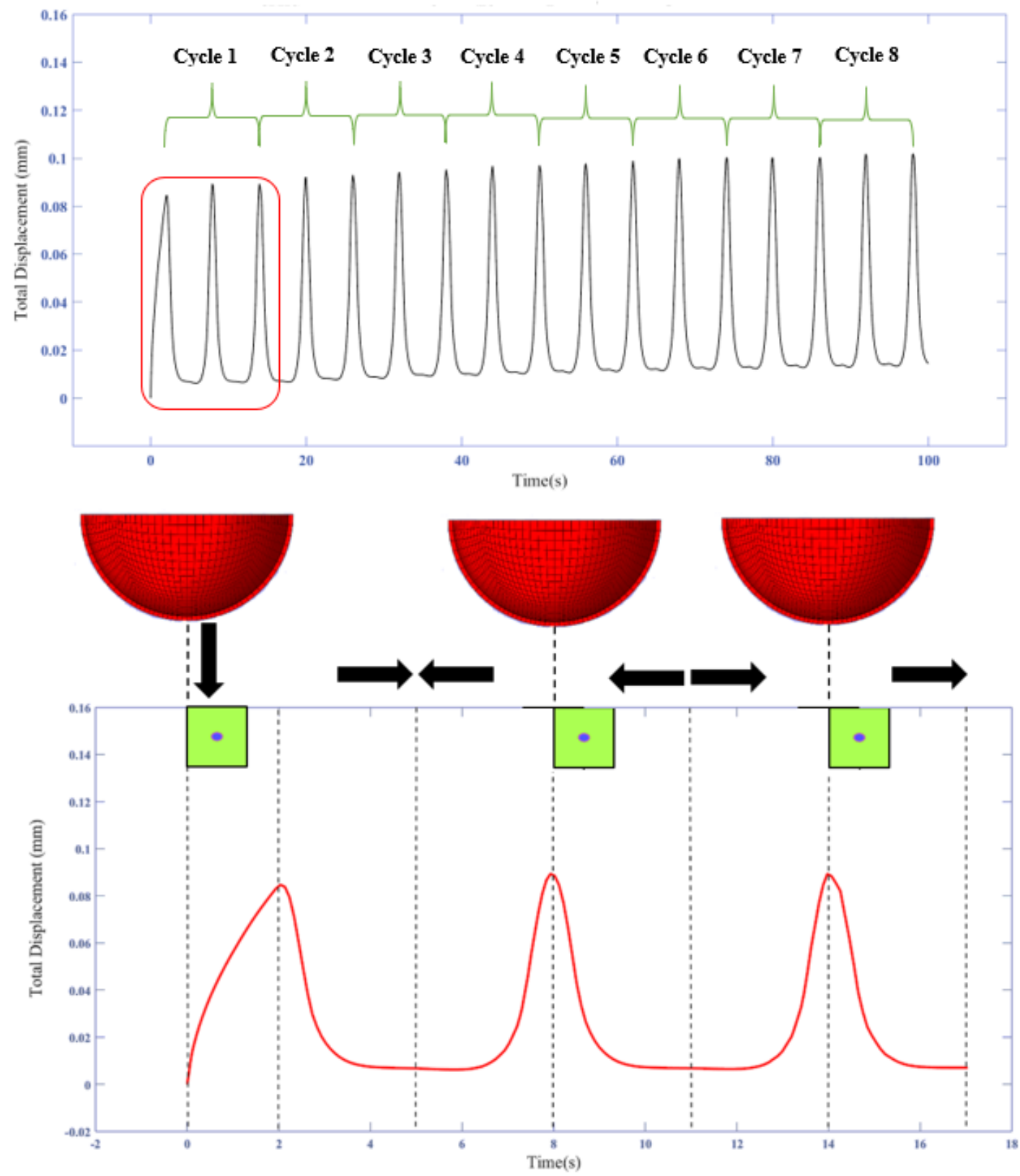

Figure 3.8: Cycle details for central microscale models in sliding indentation. The total displacement for superficial zone is shown as an example. A total of eight cycles within the simulated time are shown (top) with their respective durations. The exploded view is shown (bottom) for the first complete cycle of sliding motion including the indentation step (red) and beginning of cycle 2 , with arrows indicating the movement of the indenter movement direction in different time periods separated by dotted lines. The position of the indenter relative to the micromodel (yellow) is shown at selected time points. Model size is not shown to scale. Refer to Figure 3.2 for further details on loading protocols. 

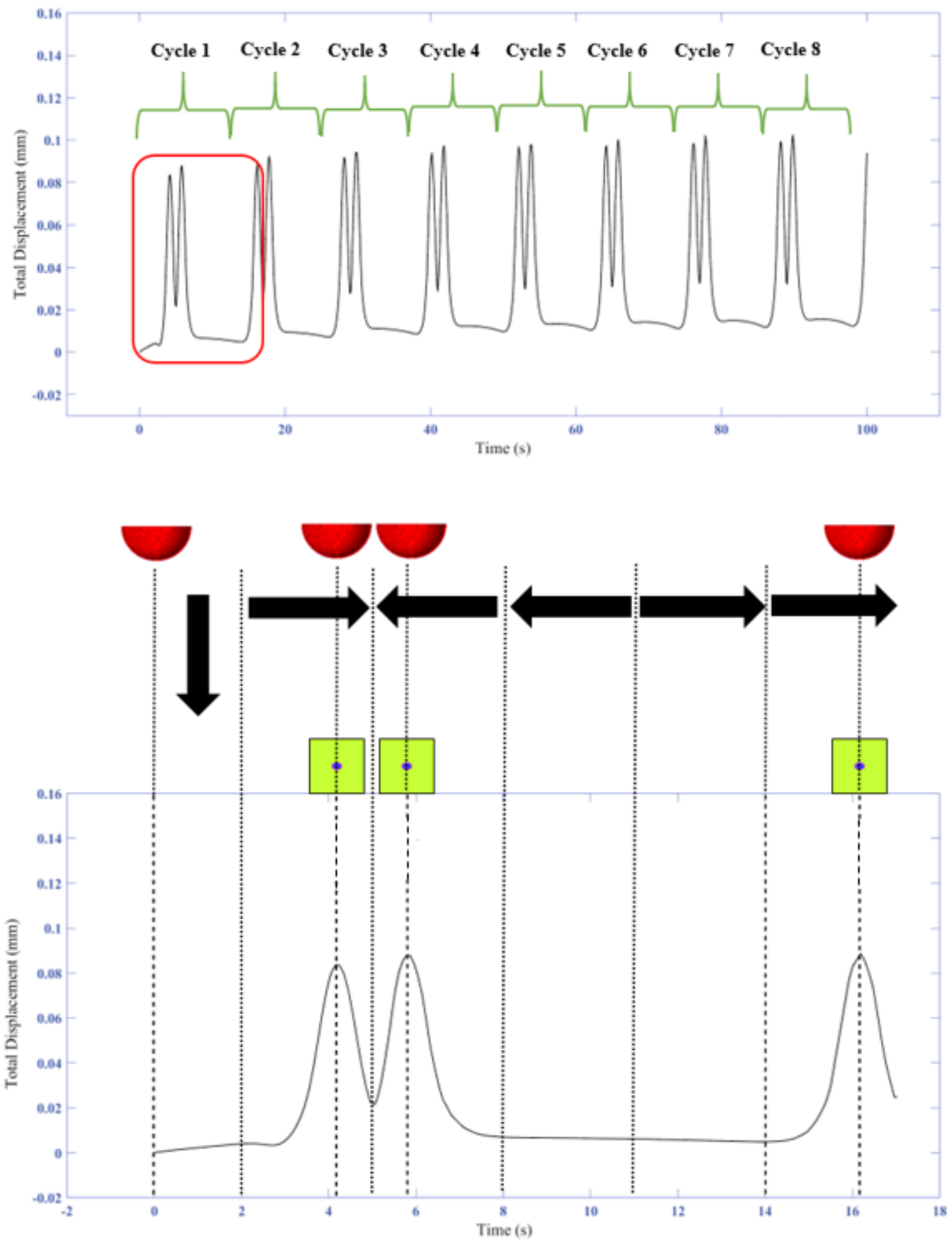

Figure 3.9: Cycle details for peripheral micromodels in sliding indentation. The total displacement for superficial zone is shown as an example. A total of eight cycles within the simulated time are shown (top) with their respective durations. The exploded view is shown (bottom) for the first complete cycle (red) and beginning of cycle 2 , with arrows indicating the indenter movement direction in different time periods separated by dotted lines. The position of the indenter relative to the micromodel (yellow) is shown at selected time points. Model size is not shown to scale. Refer to Figure 3.2 for further details on loading protocols. 
As described in Figure 3.2, the initial loading phase, i.e. 5\% ramp load applied over 2 seconds were the same for both cyclic sliding indentation and axial quasi-static indentation. Due to this, the peaks at this time point were the same for both loading modes and thus will be reported but not used for comparison purposes. When comparing between these two loading modes of healthy cartilage (Section 3.3), the averaged maxima reported for cycle $8(\approx 100$ seconds) in cyclic sliding indentation were used to compare with the peak value reported at 100 seconds for axial quasi-static indentation.

\subsection{Results}

This chapter will analyze the micromechanical environment of healthy cartilage and compare the above-mentioned variables between axial quasi-static indentation and a novel and more physiological cyclic sliding indentation. The differences between these loading modes were evaluated at the end of the sliding test, i.e. at $\approx 100$ seconds and at the same time for the quasi-static test. It will be later discussed in Chapter 4, how these strain magnitudes may differ with cartilage degeneration, i.e. OA properties. It should be noted that the general behaviour of the healthy macroscale model is shown in Appendix C.3. The compressive principal stresses and strains (Figure C.3) and fluid pressurization (Figure C.4) are shown for selected time points under physiological cyclic sliding indentation.

\subsubsection{Healthy Cartilage Under Various Loads}

Principal Strains

In all microscale models (A-F) in healthy cartilage under both cyclic sliding and axial quasi-static indentation, the $1^{\text {st }}$ principal strain remained mainly positive (tensile) for the ECM, PCM, and chondrocyte regions. In both loading modes, strain 
amplifications were found in the chondrocytes relative to the PCM and ECM regions in all microscale models. In general, the tensile strain decreased with cartilage depth, i.e. from superficial to deep zone for all regions at both locations under cyclic sliding indentation but only the central location under axial quasi-static indentation.

In microscale models subjected to cyclic sliding indentation, the averaged maximum tensile strains of cycle 8 ( $\approx 84$ to 100 seconds), showed minor differences in magnitudes between the central and peripheral locations of each zone (Figure 3.10). Under sliding loads, within the same microscale model, the largest tensile strain occurred in the chondrocyte region. Their averaged magnitudes at the central location were $9.35 \%$, $5.80 \%$, and $2.57 \%$ for the superficial, middle, and deep zones respectively, which were very similar to the magnitudes found at the peripheral location under this loading mode (Figure 3.10). Lastly, in all regions, the amplitudes of $1^{\text {st }}$ principal strain over time, i.e. from cycles 1 to 8 increased for all microscale models except for model "D". However, both amplitudes and magnitudes decreased with cartilage depth, i.e. from superficial to deep zone at both locations (Figure 3.10).

Unlike cyclic sliding indentation, at 100 seconds of axial quasi-static indentation, major differences were found in peak $1^{\text {st }}$ principal strains between the central and peripheral locations (Figure 3.11). At 100 seconds of axial quasi-static indentation, the peripheral chondrocytes undergo roughly $6.7 \%$ more tensile strain at the superficial zone, but $4.31 \%$ and $1.25 \%$ less tensile strain at the middle and deep zones compared to the central chondrocytes. Additionally, all regions of the microscale model D increased from 30 to 100 seconds (Figure 3.11D) with maximum tensile strain in the chondrocyte (6.68\%). These were less than $1 \%$ in the middle and deep zones. This suggests that at the 
tissue periphery, all regions within in the superficial zone are subject to high tensile strains with increased duration of static indentation. 

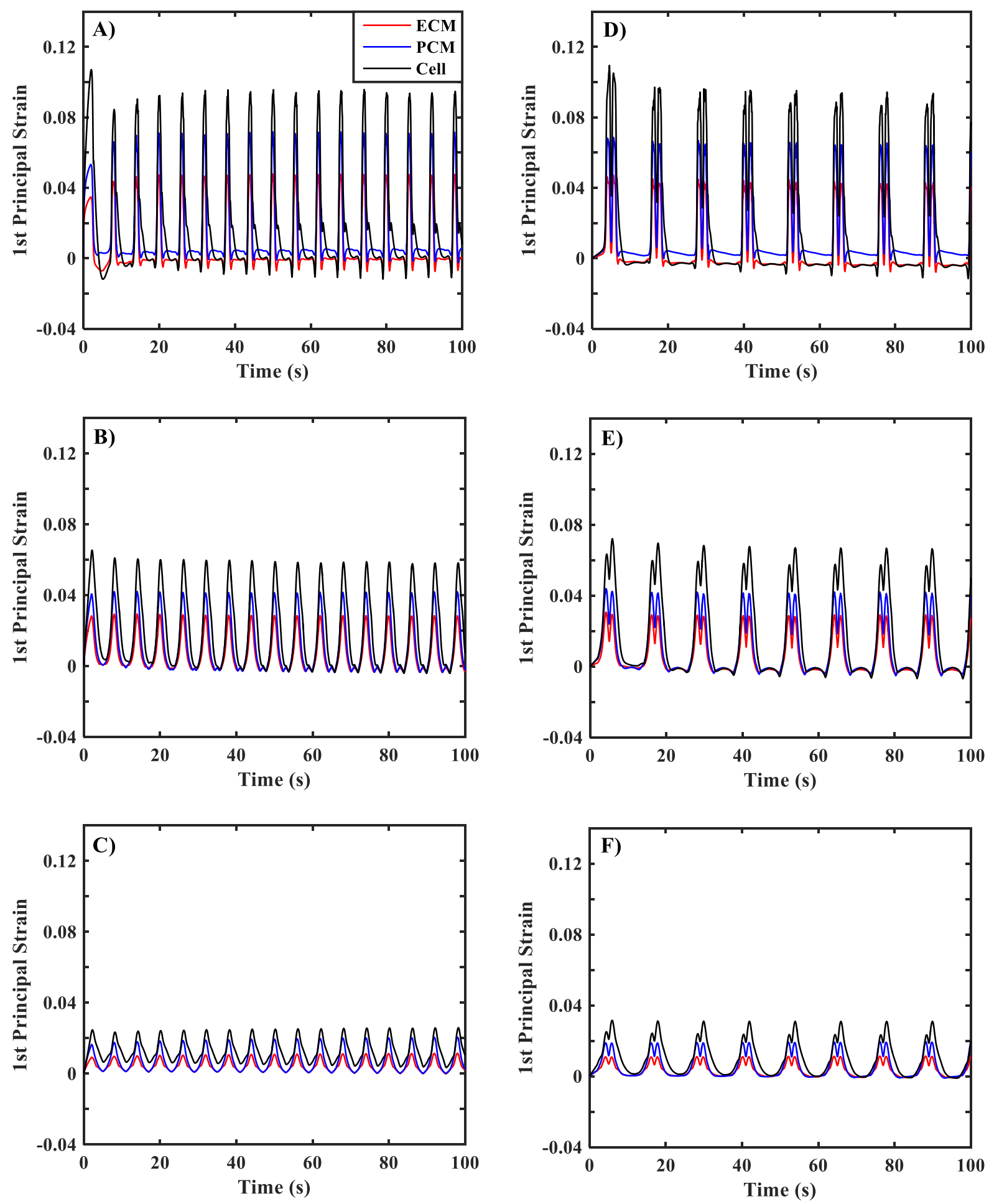

Figure 3.10: First principal strain in the microenvironment of healthy cartilage at the superficial, middle, and deep zones of the central (A-C), and peripheral (D-F) locations under cyclic sliding indentation. 

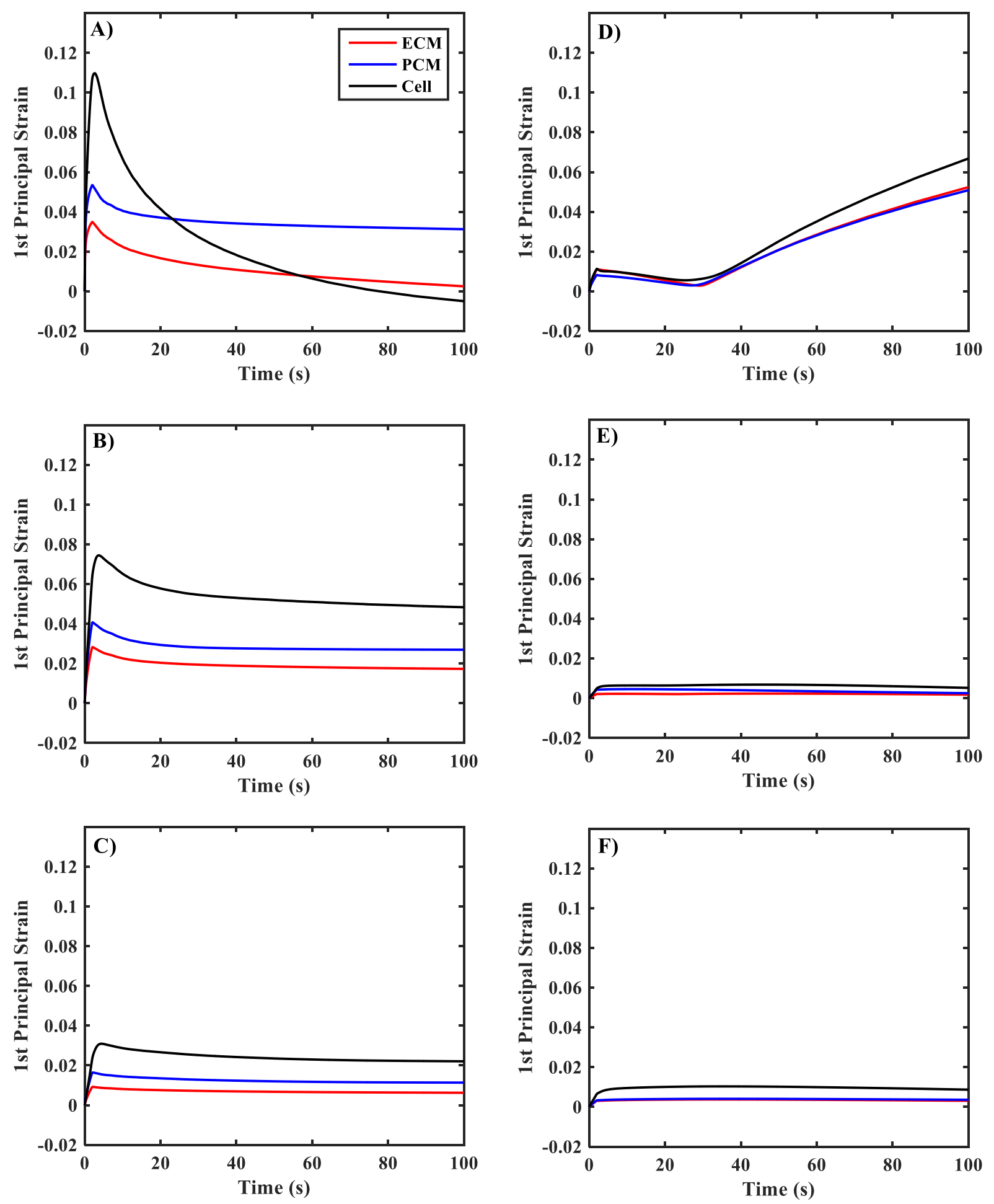

Figure 3.11: First principal strain in the microenvironment of healthy cartilage at the superficial, middle, and deep zones of the central (A-C), and peripheral (D-F) locations under axial quasi-static indentation. 
Figure 3.12 shows the magnitudes of the $1^{\text {st }}$ principal strains in each region of each microscale model in healthy cartilage as a percentage at the end of each loading mode, i.e. $\approx 100$ seconds. The peak $1^{\text {st }}$ principal strains (tensile) in all regions were lower in all microscale models under axial quasi-static indentation compared to the more physiological cyclic sliding indentation. The largest difference between these loading modes occurred in the chondrocytes at the central superficial zone and peripheral middle zone microscale models. Although Figure 3.12 only shows strain magnitudes, the differences between these loading modes for healthy cartilage will be presented for each case and further compared with OA cartilage in Chapter 4. This will be used to examine if these differences are different between cartilage grades, i.e. healthy vs. OA tissues.

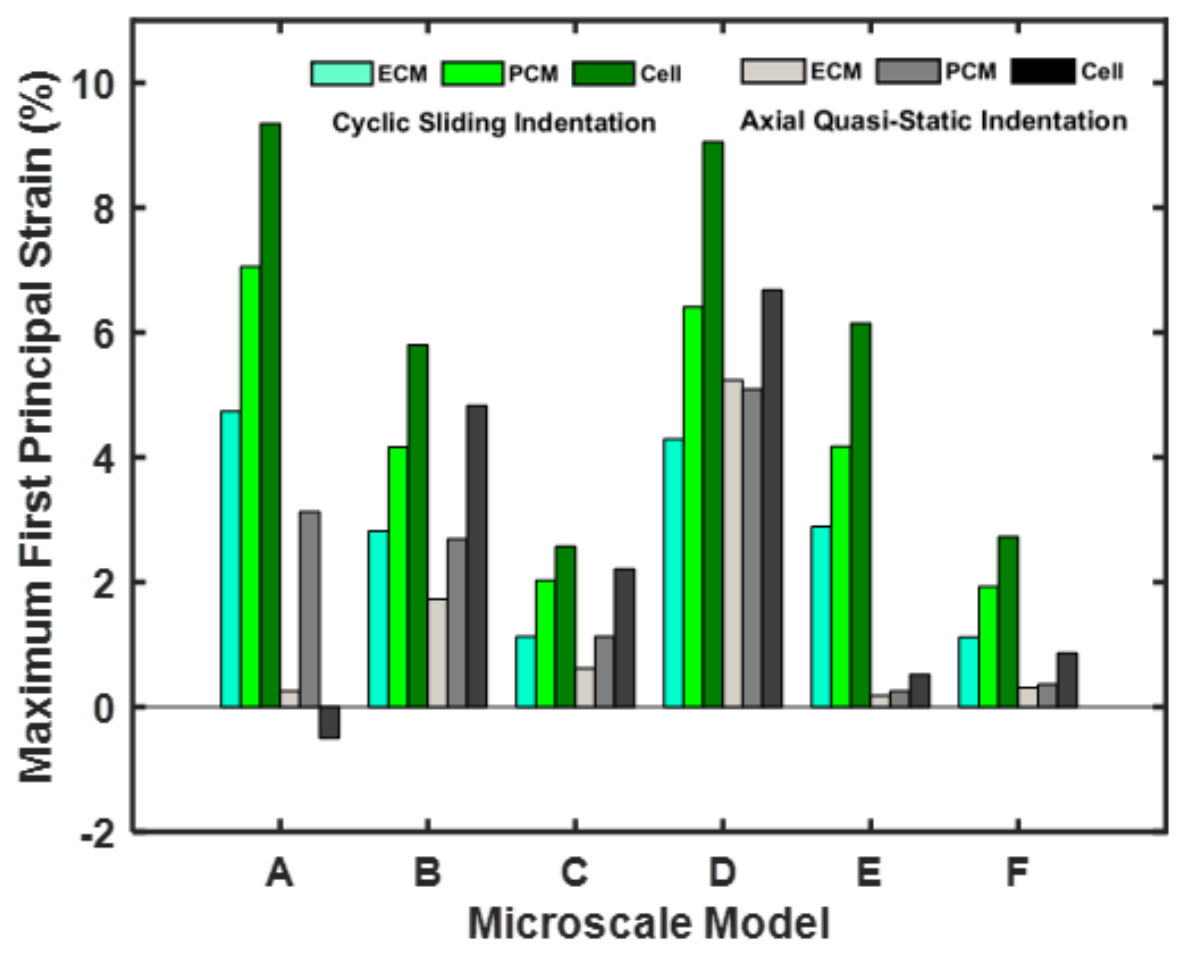

Figure 3.12: Maximum first principal strain in the microenvironment of healthy cartilage under different loading modes. 
Similarly, under both cyclic sliding and axial quasi-static indentation, the $3^{\text {rd }}$ principal strain remained mainly negative (compressive) for the ECM, PCM, and chondrocyte regions. As observed with $1^{\text {st }}$ principal strains (Figures 3.10 and 3.11), the amplifications of $3^{\text {rd }}$ principal strains were also found in the chondrocytes relative to the PCM and ECM regions in all microscale models (Figures 3.13 and 3.14). Under cyclic sliding indentation, the averaged peak compressive strains of cycle 8 showed minor differences in magnitudes between the central and peripheral locations of each zone (Figure 3.13). In the chondrocytes, the peak compressive strains at the central location were $-16.42 \%,-9.62 \%$, and $-3.56 \%$ for the superficial, middle, and deep zones respectively, which were very similar to the peripheral microscale models under this loading mode (Figure 3.13E-F).

Unlike cyclic sliding indentation, at 100 seconds of axial quasi-static indentation, large differences were observed in the peak magnitudes of the $3^{\text {rd }}$ principal strains between the central and peripheral locations (Figure 3.14). For healthy chondrocytes at the central location, these were $-42.08 \%,-19.45 \%$, and $-4.77 \%$ for the superficial, middle, and deep zones respectively. Whereas, at the peripheral location, these were $0.77 \%$, $0.38 \%$, and $-0.83 \%$ for superficial, middle, and deep zones respectively. This suggests that at 100 seconds of axial quasi-static indentation, in healthy cartilage, the central chondrocytes undergo roughly $42 \%, 19 \%$, and $4 \%$ more compressive strain at the superficial, middle, and deep zones respectively relative to the peripheral chondrocytes. 

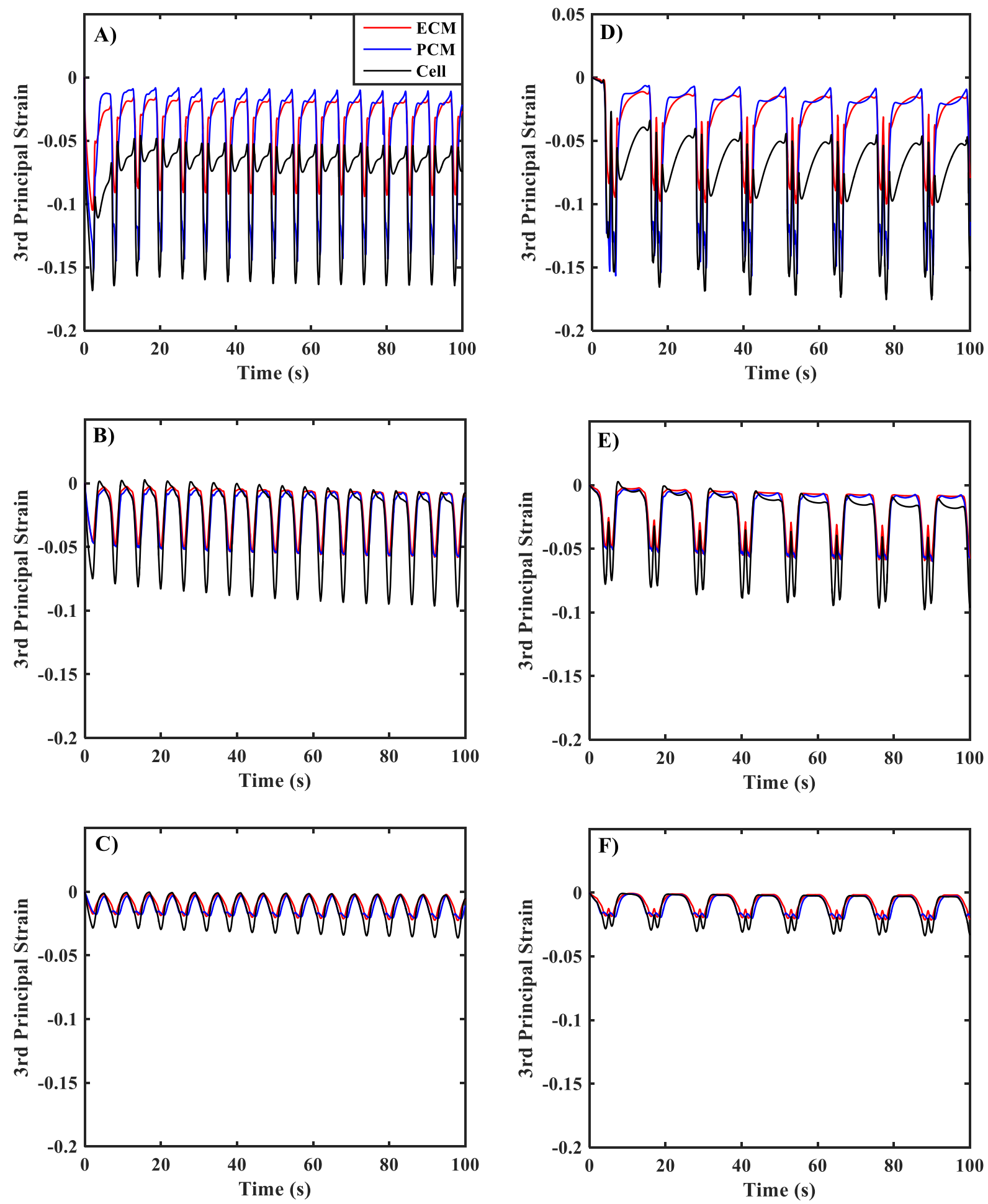

Figure 3.13: Third principal strain in the microenvironment of healthy cartilage at the superficial, middle, and deep zones of the central (A-C), and peripheral (D-F) locations under cyclic sliding indentation. 

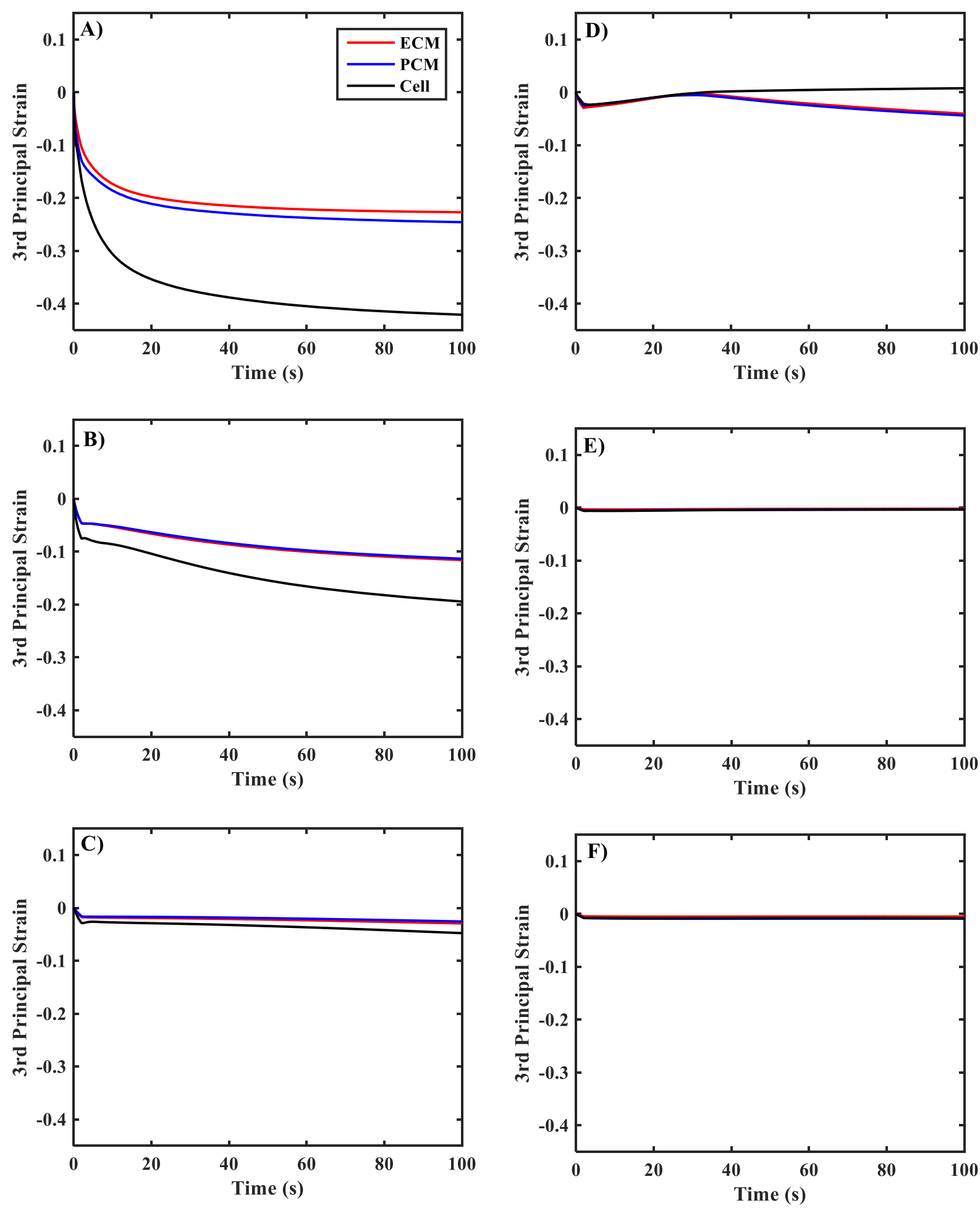

Figure 3.14: Third principal strain in the microenvironment of healthy cartilage at the superficial, middle, and deep zones of the central (A-C), and peripheral (D-F) locations under axial quasi-static indentation. 
Figure 3.15 shows the magnitudes of the $3^{\text {rd }}$ principal strains in each region of each microscale model in healthy cartilage as a percentage at the end of each loading mode, i.e. $\approx 100$ seconds. In healthy chondrocytes, the largest difference between these indentation loading modes occurred in the central superficial zone microscale model A, with the largest difference occurring in the chondrocyte region $(\approx 25 \%)$. In general, under the less physiological axial quasi-static indentation, the most compressive strains are overestimated at the central location but underestimated at the peripheral location relative to physiological cyclic sliding indentation. These differences will be further compared with degenerated cartilage (Chapter 4). This comparison is important as differences between loading modes may not be the same as between cartilage grades, i.e. healthy vs. OA.

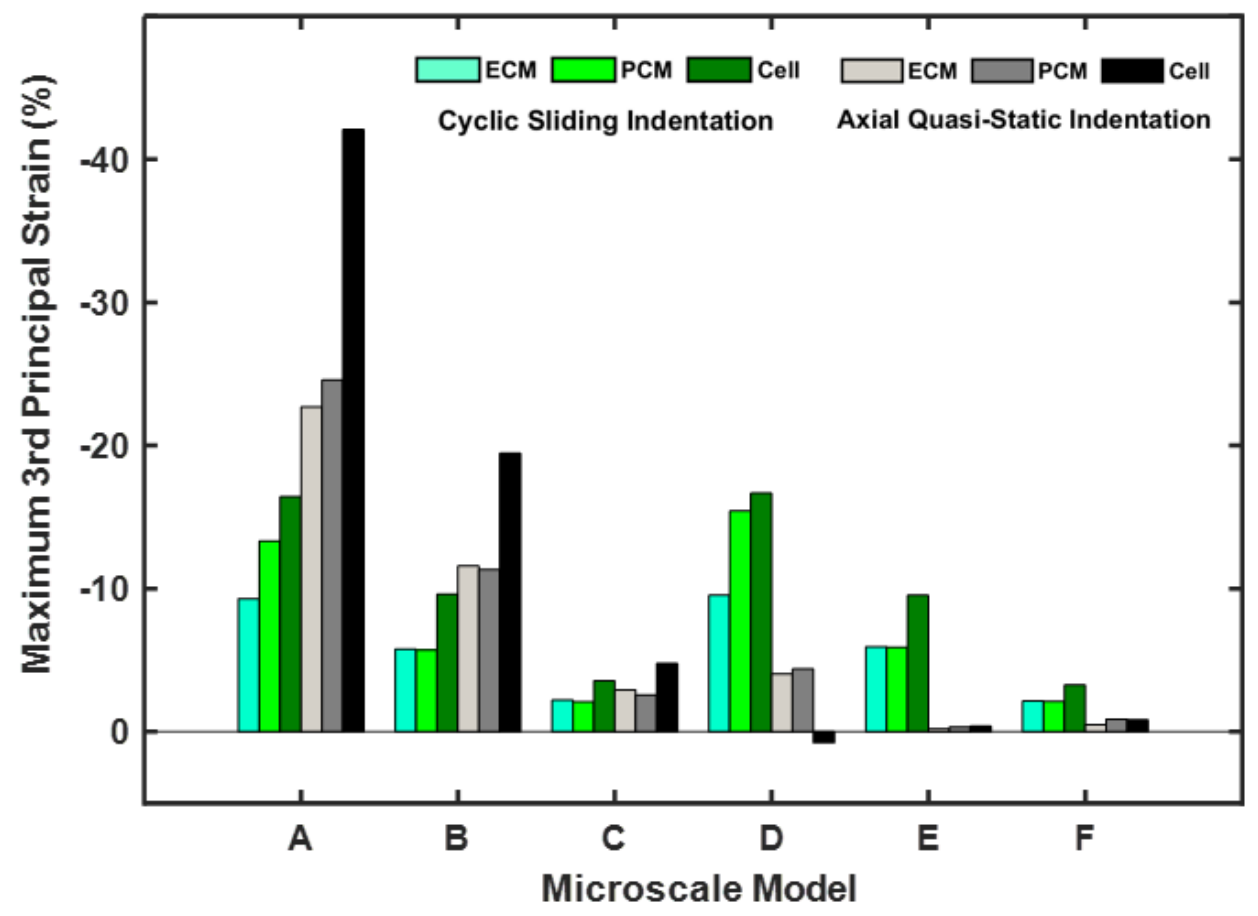

Figure 3.15: Maximum third principal strain in the microenvironment of healthy cartilage under different loading modes. 


\section{Relative Volume}

In all cases under both loading conditions, a relative volume of 1 is indicative of no change in volume. However, a value less than and greater than 1 indicates a decrease and increase in volume respectively.

Generally, the amplitudes of the relative volume generally decreased over time, i.e. from cycles 1 to 8 for all regions of all microscale models (Figure C.3). As observed with the principal strains, the amplitudes also generally decreased with cartilage depth for both locations. Furthermore, at both locations of the superficial zone, the amplitudes were largest in the PCM region (Figure C.3A and D), implying that the PCM experienced the largest range of deformation at the surface, which may contribute to their protective role on the softer chondrocytes. In the middle and deep zones at both locations, the chondrocytes had the largest amplitudes out of all regions. After the ramp load in cyclic sliding indentation, the peak volumes in all regions at the central and peripheral locations of the superficial zone always remained below 1, i.e. no increase in volume over 100 seconds (Figure C.3A and D). On the other hand, the peak volumes always remained above 1 at all times in only the PCM and chondrocyte regions at both locations of the middle (Figure C.3B and E) and deep (Figure C.3C and F) zones. Under sliding loads, the largest relative volume occurred in the chondrocytes of the middle zone $(\approx 1.05)$ at both locations (Figure C.3B and E), indicating a 5\% increase in volume.

At 100 seconds of axial quasi-static indentation, major differences in relative volume were found between the central and peripheral locations especially for the superficial, and middle zones (Figure C.4). All regions in microscale models C, E, and F and the ECM and PCM regions of microscale model D showed almost no change in 
volume. However, interestingly, the chondrocytes of microscale model D increased in volume by approximately $10 \%$ (Figure C.4D), which also happened to be the largest volume increase out of all cases in healthy cartilage. This increase in volume occurs from the same time frame (30 to 100 seconds) as observed in the increase in $1^{\text {st }}$ principal strain shown earlier in Figure 3.11. The central microscale models under axial quasi-static indentation are subject to $57.52 \%, 13.99 \%$, and $2.73 \%$ more reduction in volume in the superficial, middle, and deep zones respectively relative to cyclic sliding indentation. These results would suggest that in a less physiological axial quasi-static indentation, at the cartilage surface, the central chondrocytes experience a significantly larger reduction in volume, whereas the peripheral chondrocytes experience larger increases in volume compared to cyclic sliding indentation.

Furthermore, when loaded, the relative volume decreased and increased at the superficial zone and deep zones, respectively. These zone-dependent changes in volume were consistent with previous experimental [95] and computational $[71,76]$ studies. Guo et al. also found a similar volume trends with spherical chondrocytes implemented in all cartilage zones [71]. In this thesis, the overall volume of each chondrocyte was different due a change in aspect ratios in each zone based on dimensions acquired from a previous computational study [96]. Weather or not the difference in cellular volumes across the tissue depth is physiological has yet to be investigated. However, upon load application, this may have influenced the volume changes as it has been found in a computational study that volume deformation increased with aspect ratios in the same zone [96]. 


\section{Fluid Pressure}

When loaded, the peak fluid pressures generally decreased with cartilage depth at both locations under both cyclic sliding indentation (Figure 3.16) and axial quasi-static indentation (Figure 3.17). It was also observed that the fluid pressures were more or less uniform in the ECM, PCM, and chondrocyte regions in each individual microscale model under both loading modes. At the end of the initial ramp load, i.e. at 2 seconds, the peak fluid pressures in the central microscale models under both loading modes were 0.713 , 0.283 , and $0.127 \mathrm{MPa}$ in the superficial, middle, and deep zones respectively.

Under cyclic sliding indentation, minor differences in fluid pressures were found between the middle and deep zones of the central and peripheral location over time, i.e. from cycle 1 to 8 . However, larger differences in peak fluid pressures over time were found between the central and peripheral locations of the superficial zone. In the superficial zone, during the first cycle, the chondrocyte at the peripheral location had an averaged peak fluid pressure of $921 \mathrm{kPa}$, which was $208 \mathrm{kPa}$ more pressure than the central chondrocytes. In this loading mode, the pressure amplitudes increased slightly in almost all regions of the central microscale models over 100 seconds but decreased in all regions of the peripheral microscale models. Lastly, as observed with the principal strains and relative volume, the amplitudes for fluid pressure also decreased with cartilage depth (Figure 3.16). At 100 seconds of axial quasi-static indentation, the pressures reached below $100 \mathrm{kPa}$ for both locations (Figure 3.17). In this loading mode, the largest pressure in the chondrocytes was at the central location which was $60.9,64.6$, and $61.5 \mathrm{KPa}$ for the superficial, middle, and deep zones respectively. These were comparably much lower than under cyclic sliding indentation (Figure 3.16). 

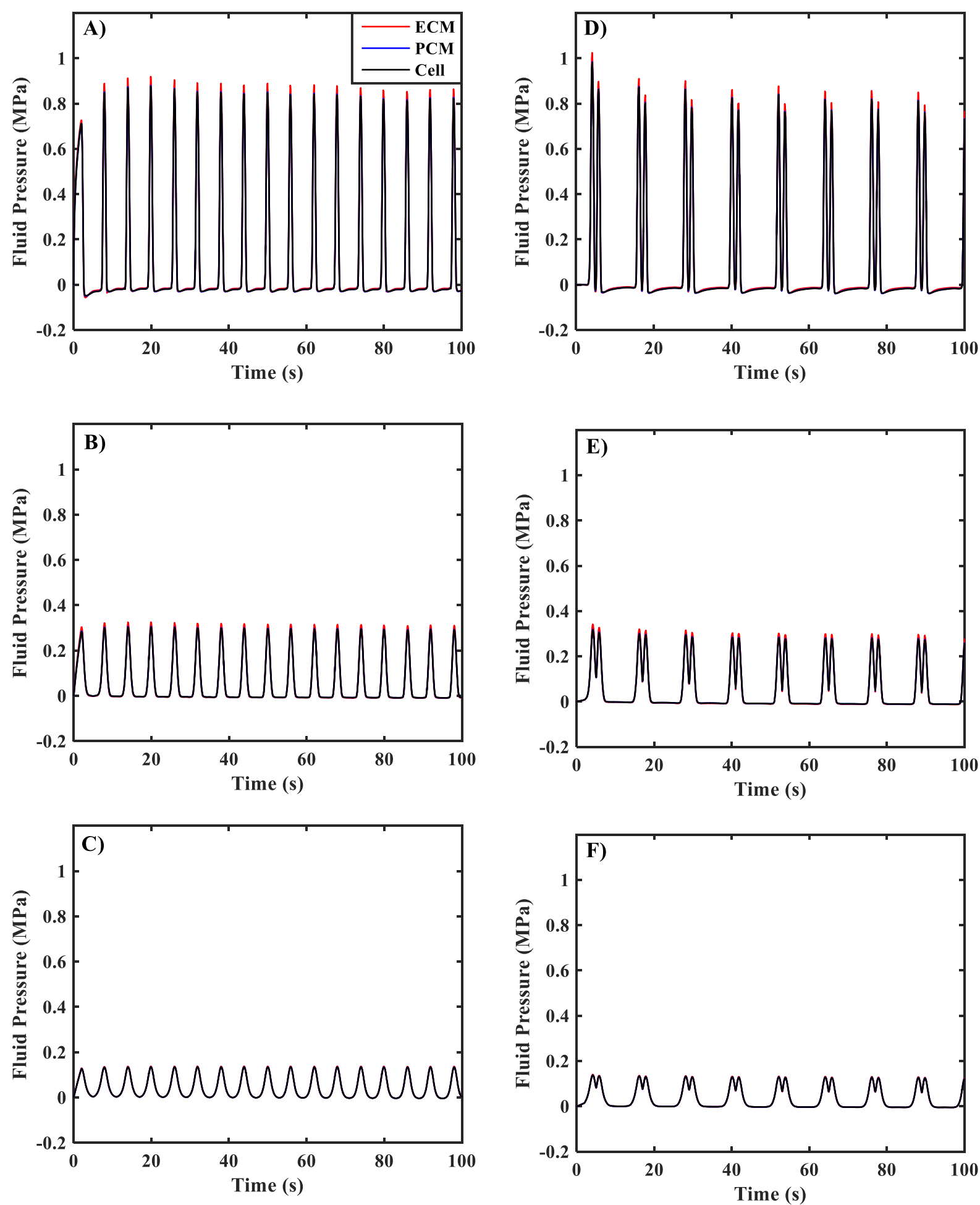

Figure 3.16: Fluid pressure in the microenvironment of healthy cartilage at the superficial, middle, and deep zones of the central (A-C), and peripheral (D-F) locations under cyclic sliding indentation. 

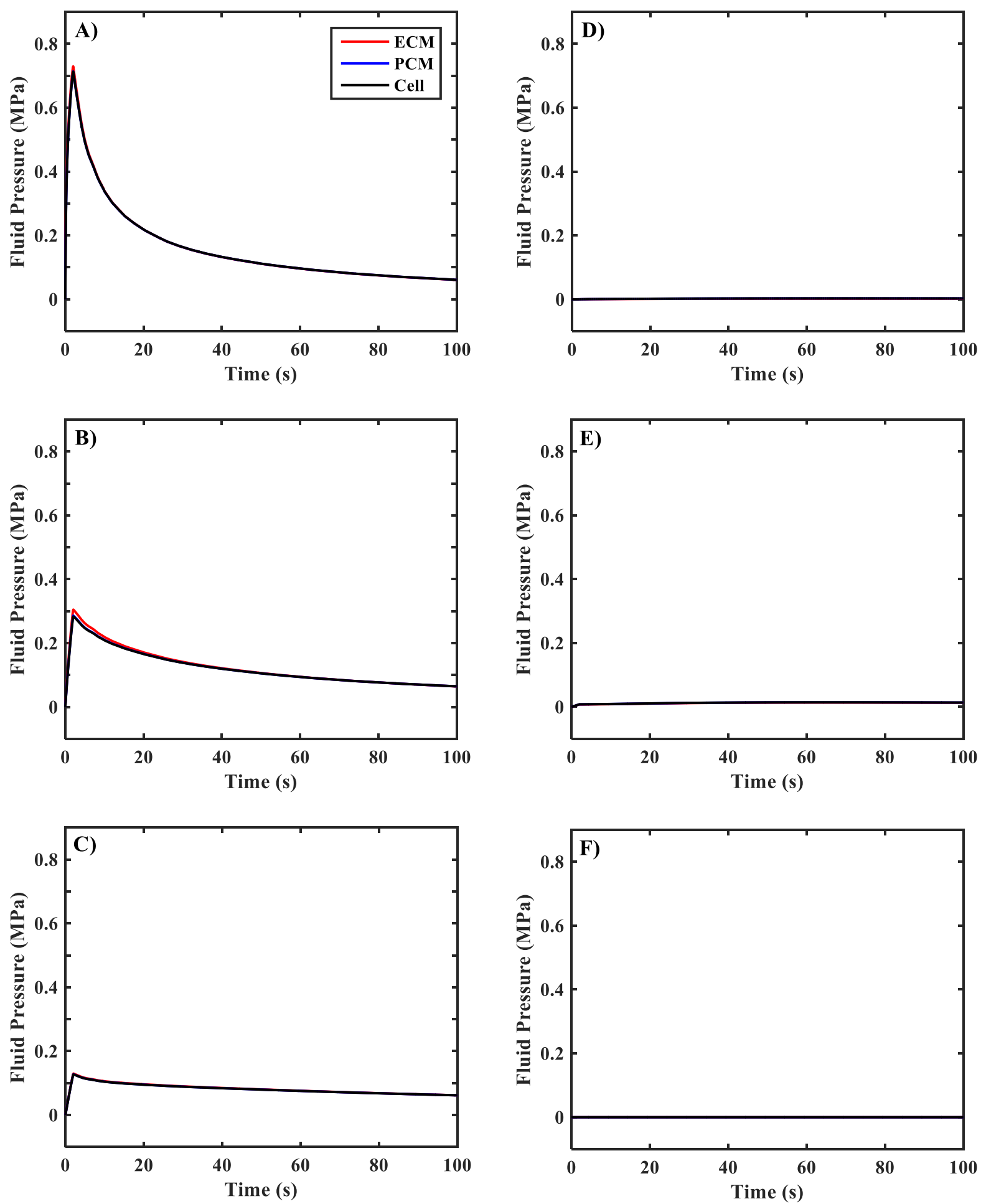

Figure 3.17: Fluid pressure in the microenvironment of healthy cartilage at the superficial, middle, and deep zones of the central (A-C), and peripheral (D-F) locations under axial quasi-static indentation. 
Figure 3.18 shows fluid pressures in all microscale models at the end of both loading modes ( $\approx 100$ seconds). It was observed that the ECM, PCM, and chondrocyte regions in all zones of both locations experience larger fluid pressures in the physiological cyclic sliding indentation relative to axial quasi-static indentation. The largest differences in fluid pressure between these loading modes were at the superficial zone microscale models at the central (A) and peripheral (D) locations. This is a useful finding, as it shows that large discrepancies exist in not only the solid but also the fluid microenvironment between these loading modes, which suggests that wrong conclusions can be made about cartilage behaviour if the wrong loading mode is applied.

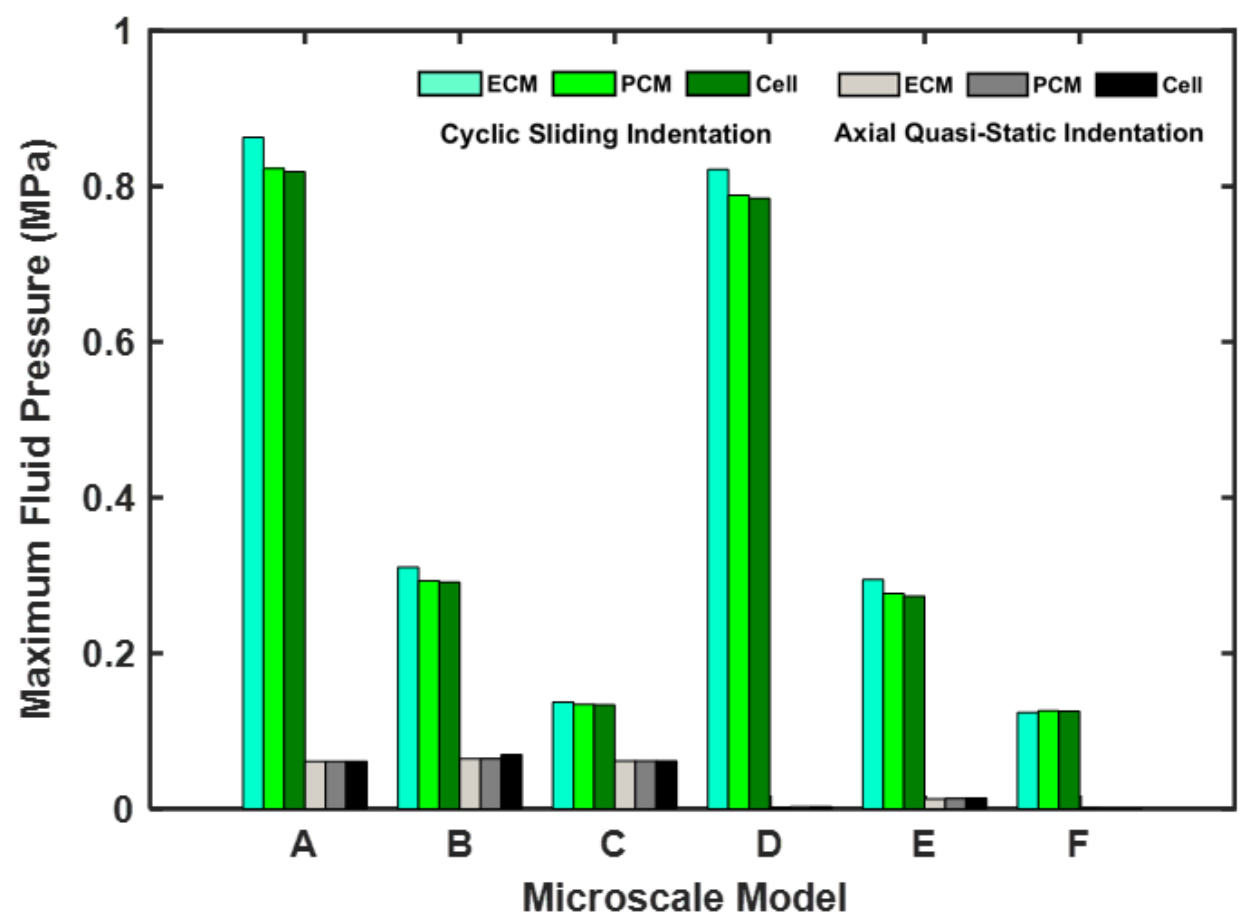

Figure 3.18: Maximum fluid pressure in the microenvironment of healthy cartilage under different loading modes. 
Fluid Flux

The peak fluid flux generally decreased with cartilage depth at both locations under both loading scenarios. Unlike fluid pressures, the fluid flux in each region, i.e. the ECM, PCM, and chondrocyte of each individual microscale model were non-uniform. In each microscale model, the flux in the ECM region was at least one order of magnitude higher relative to the chondrons in both loading modes. Furthermore, in both loading modes, flux was found to be generally higher at the peripheral location. Due to this, this location will be used for comparison purposes. The averaged flux magnitudes in the last cycle of sliding indentation in the ECM region at this location were 7800, 1600, and 420 $\mathrm{nm} / \mathrm{s}$ in the superficial, middle, and deep zones respectively. On the other hand, at 100 seconds of axial quasi-static indentation, the ECM flux magnitudes were 59, 66, and 65 $\mathrm{nm} / \mathrm{s}$ in the superficial, middle, and deep zones respectively, which were much lower than under sliding indentation.

Similarly, at the end of both loading modes, the fluid flux in the chondron under axial quasi-static indentation was also found to be lower compared to all microscale models under cyclic sliding indentation. The maximum flux differences between these loading modes were at the superficial zone, i.e. microscale models A and D. Under cyclic sliding indentation, the PCM and intracellular flux resulted to be almost 200 and 100 $\mathrm{nm} / \mathrm{s}$ higher at both locations of the superficial zone than under axial quasi-static indentation.

In healthy cartilage, along with the magnitude of flux. the direction of flux varied based on the relative position of the indenter, which was dependent on the loading protocol. The vector plot for cyclic sliding indentation is shown in Figure C.7 in 
Appendix C. It should be noted that the arrows of the vector were scaled for better visualization and are not proportional to the flux magnitudes.

During the loading phase from 0 to 2 seconds (Figure C.7a), the flux direction in the central microscale models (A-C) was pointed downwards since these models lay directly underneath the indenter. As the indenter started to slide along the $\mathrm{x}$-axis, the flux direction was always in the opposite direction. For simplicity, the vector plots in (Figure C.7b) only show the indenter sliding in the positive $\mathrm{x}$-direction for a quarter of the first cycle. Once the slider had completely translated off the microscale models at the unloaded phase (Figure C.7c), the flux direction of microscale models A and B generally pointed upwards (positive z-direction). However, in model C, the flux never points in the upward direction, rather it remains along the $\mathrm{x}$-axis depending on the relative $\mathrm{x}$-position of the indenter as mentioned above. These fluid flux patterns were similar in microscale models at the peripheral location in their respective loaded and unloaded phases.

Due to the differences in loading protocol after 2 seconds, the direction of fluid flux differed in the axial quasi-static indentation (Figure C.8) compared to cyclic sliding indentation (Figure C.7). At the end of ramp load at 2 seconds, the flux vector pointed downwards (-z-direction) for the central models (A-C), as observed in sliding indentation. Since the middle zone microscale models B and E had similar flux patterns compared to the superficial zone, they were not shown in Figure C.8. When the load is held constant over 100 seconds, the flux vector points upward in the superficial and middle zones of the central location, whereas, it remained in the downward direction in the central deep zone. Like the unloaded phases in sliding indentation, the flux vector in axial quasi-static indentation was pointed towards the top of the tissue for superficial and 
middle zones of the peripheral location. However, unlike sliding indentation, this direction was maintained for the remaining 100 seconds of axial quasi-static indentation. Lastly, in the peripheral deep zone microscale model, the flux vector is always directed along the positive $\mathrm{x}$-direction from 2 to 100 seconds.

\section{Maximum Chondrocyte Shear Stresses and Strains}

In healthy cartilage, the maximum shear stresses and strains generally decreased with cartilage depth at both locations under both loading modes. Minor differences in shear stresses and strains were found between zones of central and peripheral locations under cyclic sliding indentation. Major differences were found between these locations under axial quasi-static indentation. At the end of both loading modes, maximum shear strains under axial quasi-static indentation were found to be larger in the microscale models at the central location, but smaller at the peripheral location compared to cyclic sliding indentation, as shown in Figure 3.19.

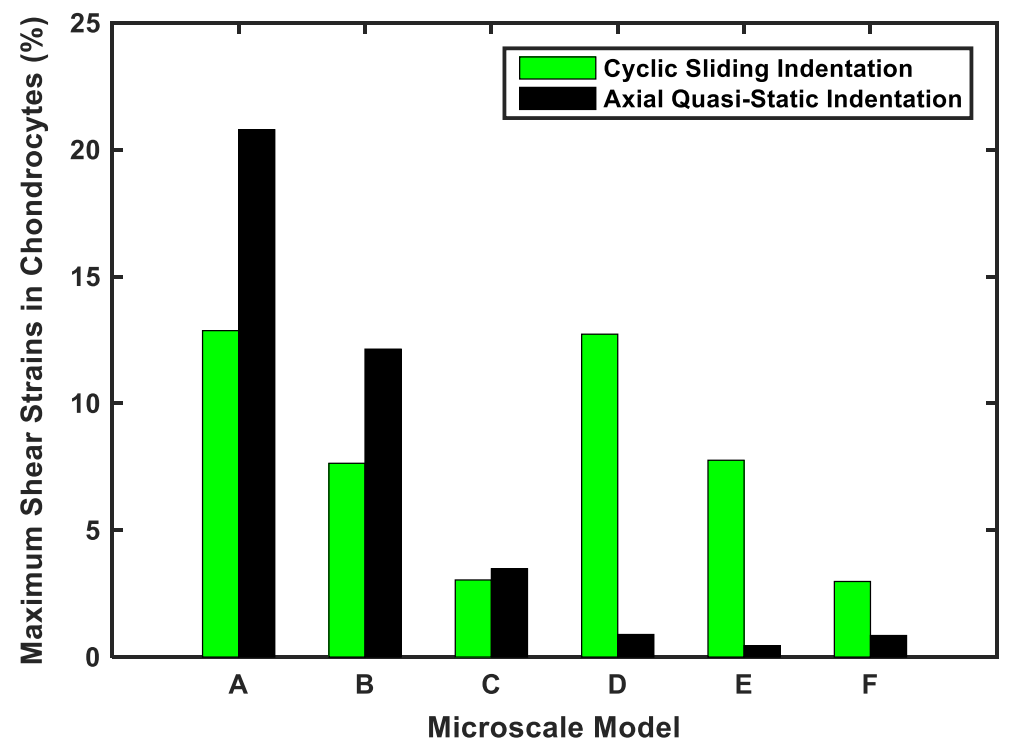

Figure 3.19: Maximum shear strains in healthy chondrocytes under different loading modes. 


\subsection{Discussion}

In this chapter, the micromechanical environment of healthy cartilage was investigated at six different locations under both axial quasi-static and cyclic sliding indentation. This was achieved using BPVE fibril-reinforced multiscale finite element models with depth-dependent material properties and shape of chondrons. It was found that both loading modes and spatial locations had an impact on the solid stresses/strains, and fluid pressures/fluxes in the ECM, PCM, and chondrocyte regions. The analysis of healthy cartilage performed in this chapter will be later compared to degenerated (OA) cartilage presented in Chapter 4. It should be noted that although the location of the ECM node studied in this thesis (Figure 3.7) is largely driven by the imposed boundary conditions, the comparisons of strains and other outcome variables shown in Section 3.3 also depended on the variation in material properties. Also, at present, no study has investigated the micromechanical environment with sliding indentation, therefore no validation can be provided for this loading mode.

\section{Principal Strains}

As noted in Section 3.3.1, in healthy cartilage under both loading modes, the $1^{\text {st }}$ principal strains (Figure 3.10 and 3.11 ) remained mostly positive and $3^{\text {rd }}$ principal strains (Figure 3.13 and 3.14) remained mostly negative, implying that these were tensile and compressive strains respectively. In both cyclic sliding and axial quasi-static indentation, the magnitudes of both principal strains decreased from the superficial to deep zones of cartilage. Although no study has performed a biomechanical analysis at the microenvironment using the novel cyclic sliding indentation, this finding was consistent with a previous depth-dependent computational study on microscale cartilage performed 
under unconfined static compression [71]. Since the principal strains are amplified at the cartilage surface, this may suggest that the superficial zone has a protective role for deeper zones. In addition to strain differences with zonal locations, the central and peripheral locations also influenced the strain magnitudes. This was particularly noticeable between the two different loading modes. In axial quasi-static indentation, both the $1^{\text {st }}$ and $3^{\text {rd }}$ principal strains in the peripheral models (D-F) were significantly smaller in magnitude compared to cyclic sliding indentation. This is because the peripheral models were stimulated by the migrating contact area of the indenter in the sliding motion. For axial quasi-static indentation in this study, this was a novel finding in comparison to a study on static loading by Guo et al., which showed that in a state of unconfined compression, both central and peripheral models had similar strain magnitudes [71] which unlike indentation, provides a global stimulus to the tissue. In general, the indentation loading mode provides a more local stimulus to the tissue which better represents the physiological conditions of cartilage in in-vivo [100]. Moreover, in this study, the results also showed time-dependent changes, since both loading modes were evaluated over 100 seconds. For cyclic sliding indentation, in the same zone, there were negligible differences in strain magnitudes over time for both central and peripheral locations for reasons mentioned above. Whether or not these changes would become more significant over a large duration of time (>100 seconds) will be something to investigate in the future. In the case for axial quasi-static indentation, a general decrease and increase in magnitudes of the $1^{\text {st }}$ and $3^{\text {rd }}$ principal strains respectively were observed over 100 seconds. 
Another aspect of this chapter focused on the differences between these two loading modes in healthy cartilage tissue. The peak $1^{\text {st }}$ principal strains (tensile) in all regions were lower in all microscale models under axial quasi-static indentation compared to the more physiological cyclic sliding indentation. This is most likely due to the differences due to the relative motion of the indenter since in both loading protocols the load was held constant for 100 seconds. The physiological sliding motion generates a distributed strain field which was not observed in the axial quasi-static indentation. With this finding, it can be concluded that under 100 seconds of static indentation, the $1^{\text {st }}$ principal strains in both locations of all cartilage zones are underestimated compared to physiological cyclic sliding indentation. Similarly, the peak $3^{\text {rd }}$ principal strains (mainly compressive) under axial quasi-static indentation in all regions seemed to be overestimated in the microscale models at the central location but underestimated at the peripheral location compared to the sliding indentation. As mentioned earlier, the overestimation resulted from the central microscale models remaining under the stationary position of the indenter. The central position of the indenter also resulted in the underestimation of $3^{\text {rd }}$ principal strains at the peripheral location due to the lack of local stimulation at the tissue periphery.

\section{Relative Volume}

In this study, the volume changes in cyclic sliding (Figure C.3) and axial quasistatic indentation (Figure C.4) were zone-dependent, as found in previous studies on static loading $[71,76]$. At the initial ramp phase of both loading modes, the chondrocytes of the middle zone of the central chondrocytes had the maximum volume increase of $5 \%$. This finding was consistent with a study by Guo et al., which showed that the deeper 
zones had larger volume increases relative to the superficial zone at the initial ramp phase [71]. The volume changes are due to the competition between the $1^{\text {st }}$ (mainly tensile) and $3^{\text {rd }}$ principal (mainly compressive) strains and stresses [71]. The increase and decrease in the volume are due to the tensile and compressive stresses and strains respectively [71]. Furthermore, as expected, minor differences in volume between central and peripheral locations of the same zone under cyclic sliding indentation. Whereas, in the axial quasistatic indentation, almost no change in volume is observed in at least the middle and deep zones of the peripheral location. This was expected since the static central position of the indenter did not enforce large tensile or compressive strains at the peripheral locations.

Comparing volume magnitudes between loading modes, the central chondrocytes under 100 seconds of axial quasi-static indentation experienced $57.52 \%, 13.99 \%$, and $2.73 \%$ more reduction in volume in the superficial, middle, and deep zones respectively relative to cyclic sliding indentation. These volume decreases were expected since the indenter remained at the center of the tissue for 100 seconds, which amplified the compressive strains of all regions of these central models relative to the initial ramp load. These are useful findings as increased cellular volume could make the region more permeable and therefore may improve transport of nutrients and fluids from the ECM to chondrocytes [71].

Furthermore, the strain energy density (SED) can be calculated to show the homogenization between the macro-and microscales. This can be achieved by using the 3D principal stresses and strains. The SED for selected models, i.e. A and C at selected time points for each scale are summarized in Table 3.4. The percent differences for model 
A between these scales at 50 and 86 seconds were $6.42 \%$ and $30.65 \%$, respectively.

Whereas, for model $\mathrm{C}$, these were $2.87 \%$ and $2.28 \%$, respectively.

Table 3.4: Strain energy densities in selected macro- and micromodels of healthy cartilage under cyclic sliding indentation.

\begin{tabular}{lll}
\cline { 2 - 3 } & \multicolumn{2}{c}{$* \operatorname{SED}\left(\mathrm{KJ} / \mathrm{m}^{3}\right)$} \\
\cline { 2 - 3 } & \multicolumn{1}{c}{$\mathrm{t}=50 \mathrm{~s}$} & $\mathrm{t}=86 \mathrm{~s}$ \\
\hline Macroscale Model A & 48.30 & 36.50 \\
Microscale Model A & 45.20 & 25.31 \\
Macroscale Model C & 0.9542 & 1.095 \\
Microscale Model C & 0.9268 & 1.070 \\
\hline *Denotes strain energy density and is calculated by; \\
SED $=\frac{1}{2}\left(\left(\sigma_{1} \varepsilon_{1}\right)+\left(\sigma_{2} \varepsilon_{2}\right)+\left(\sigma_{3} \varepsilon_{3}\right)\right)$
\end{tabular}

Fluid Pressures and Fluxes

Peak fluid pressures and fluxes generally decreased with cartilage depth in all microscale models of cyclic sliding indentation (Figure 3.16), and central models of axial quasi-static indentation (Figure 3.17). The pressure drops from the superficial to deep zone occurred since the superficial zone was much closer to the load applied by the indenter and therefore the contact and fluid pressures were higher at the surface of the tissue. Minor pressure differences occurred between central and peripheral locations in cyclic sliding indentation, whereas major differences were found under axial quasi-static indentation. This was simply due to the centered position of the indenter at the midline of the tissue which stimulated only the local region and therefore did not induce high pressures at the tissue periphery, even over 100 seconds of constantly applied load. Fluid fluxes also followed these trends since pressures are proportional to the flux based on Darcy's Law given in equation 1 . The behaviour of the fluid microenvironment under 
both indentation loads was novel since static loading in the more common unconfined compression resulted in similar fluid pressures and fluxes in both central and peripheral locations [71]. The fluid pressures were also roughly the same in the ECM, PCM, and chondrocyte regions within each microscale model in both loading modes (Figures 3.16 and 3.17) as the data curved for these regions overlap each other. This was to be expected, even with discrepancies in the permeabilities of these regions. However, the solid strains and volume changes were noticeably different in these regions. For instance, the chondrocyte of microscale model D under axial quasi-static indentation had the largest volume increase (10\%) from Figure C.4, which was distinguishable from the ECM and PCM regions, however, the fluid pressures remained the same in all regions and were at a minimum $(100 \mathrm{kPa})$ as shown in Figure 3.17. This was similar to a computational study which showed that changing the aspect ratio of chondrocytes in the same zone affected the solid but not the fluid microenvironment [96]. The significance of this finding can be tied back to chondrocyte mechanobiology studies discussed in Chapter 5.

The fluid flux in all regions of all microscale models under cyclic sliding indentation was larger compared to axial quasi-static indentation. At the end of both loading modes, the flux in the ECM under sliding indentation was two orders of magnitude larger in the middle and deep zones compared to axial quasi-static indentation. Between these loading modes, the largest flux difference in the chondron occurred in the superficial zone. This was to be expected as the indenter in sliding indentation was moving at a constant velocity of $1 \mathrm{~mm} / \mathrm{s}$ over 100 seconds, generating a pressure gradient across the tissue and allowing more fluid flow. However, in axial quasi-static indentation, 
this flow was hindered due to the static position of the indenter. The averaged flux magnitudes in the central chondrocytes at 100 seconds of axial quasi-static indentation were approximately in the range of $1-100 \mathrm{~nm} / \mathrm{s}$, which was also found in a similar computational study [123]. As mentioned in Chapter 1, with low permeability of cartilage, the flow was approximated to be laminar and governed by Darcy's Law as given in equation 1 . However, the laminar flow approximation can be confirmed with the Reynold's number less than 1 . This can be calculated with the density of water (997 $\left.\mathrm{kg} / \mathrm{m}^{3}\right)$, viscosity of water $\left(8.9 \times 10^{-4} \mathrm{Ns} / \mathrm{m}^{2}\right)$, the cartilage thickness as the characteristic length $(0.002 \mathrm{~m})$, and a representative ECM velocity. The latter can be chosen for the central location microscale model at the tissue surface, which was reported as $7900 \mathrm{~nm} / \mathrm{s}$ in Table A.1 in Appendix A. With these parameters, the Reynolds number was calculated to be 0.018 which justifies the laminar flow assumption.

The direction of fluid flux varied based on the tissue zone and loading mode as shown earlier in Figures C.7 and C.8 in Appendix C. For both loading modes, the superficial and middle zones had similar flux patterns compared to the deep zone. However, differences were also observed in the peripheral models of axial quasi-static indentation but not under cyclic sliding indentation. In sliding indentation, both central and peripheral models for the same zone had similar fluid flux patterns in their respective phases of the indenter loading, sliding, and unloading on these models. In superficial and middle zones of sliding indentation, the general pattern was that the flux pointed in the downward (-z-direction) when loaded and pointed generally in the upward (+z-direction) when unloaded since the top face of the tissue was a free-draining boundary. Immediately after the loading phase, when the indenter slid along the x-axis, the fluid flowed in the 
opposite direction. These directions were reasonable since the fluid wanted to flow from areas of high to low pressures, as indicated by the minus sign in Darcy's Law in equation 1. It should be noted that at the furthest $\mathrm{x}$-distance between the indenter and the superficial zone models in sliding indentation, fluid eventually flowed back into the tissue (-z-direction) but was not shown in Figure C.7. Interestingly, the flux direction in the deep zone models during their respective unloaded phases of sliding indentation, the fluid flowed generally along the $\mathrm{x}$-axis depending on the x-position of the indenter during each cycle. In axial quasi-static indentation, for the central models, the fluid flow was the same as sliding indentation for the initial loading phase for the superficial and middle zone, but after 100 seconds, it switched to the upward direction (Figure C.8). This was presumably because these zones were relatively closer to the free-draining boundary at the surface, and therefore the fluid gradually flowed out from the loaded region and eventually out of the permeable surface of the tissue over time. In the central deep zone model, the direction of fluid flow remained downwards for 100 seconds. The peripheral models in axial quasi-static indentation were never directly loaded by the indenter, and so the fluid flowed in the upward direction in the superficial and middle zones, whereas it flowed in the positive x-axis in the deep zone. The flow followed these directions since the upper zones in the peripheral location of the tissue were still closer to the top freedraining boundary rather than the right periphery. In the deep zone, the flow was restricted in the $\mathrm{x}$-axis as seen in the sliding indentation models but remained in this direction for 100 seconds.

Maximum Chondrocyte Shear Stresses and Strains 
The maximum shear strains and stresses in healthy cartilage followed the similar trends as the $3^{\text {rd }}$ principal strains. As seen with $3^{\text {rd }}$ principal strain, it was concluded that shear stresses and strains overestimated at the tissue center and underestimated at the tissue periphery under axial quasi-static indentation relative to the more physiological cyclic sliding indentation. As explained earlier, this was because the sliding motion of the indenter provided a local stimulation across the tissue as opposed to a small region at the central location as seen in under axial quasi-static indentation. 


\section{Biomechanical Analysis of the Microenvironment of OA Cartilage}

\subsection{Introduction}

In Chapter 3, a novel physiological cyclic sliding indentation was introduced and compared with a more common axial quasi-static indentation studied in the past [98]. This was performed using six novel BPVE fibril-reinforced FE microscale models to examine the influence of both loading conditions on the microenvironment, i.e. the ECM, PCM, and chondrocyte regions of healthy cartilage. These microscale models were designed with inhomogeneous material properties arising from the superficial, middle, and deep zones of the central and peripheral spatial locations. From Chapter 3, the most influential result variables studied were the $1^{\text {st }}$ and $3^{\text {rd }}$ principal strains, relative volume, fluid pressures and fluxes for all regions and intracellular shear stresses and strains. The choice of these variables was based on a combination of experimental [78] and computational $[71,77]$ studies, described in Section 3.2.3. In this chapter, the same variables will be considered for all OA microscale models. Additionally, the locations of the three spatial nodes representing the ECM, PCM, and chondrocyte regions shown earlier in Figure 3.7 of Section 3.2.3 will be the same for OA cartilage in this chapter. The goal of this study was to assess OA cartilage under the novel physiological sliding model, and to compare the results with healthy cartilage.

\subsection{Methods}

As done in Chapter 3, both macroscale and microscale models consisted of the same discretization of the continuum, material types, and loading protocols. In this chapter, these models will differ in material properties representing early degenerative changes in 
cartilage tissue. These were first applied to the macroscale tissue, to acquire loadcontrolled boundary conditions associated with OA changes, before being assigned to the microscale models.

In the macroscale model, Young's Modulus (E) of the solid matrix was reduced by $15 \%$ to represent the depletion of PG content (Figure 1.4), and the permeability (k) was increased by $20 \%[41,125]$. Collagen fibrillation was simulated by a decrease in fibril stiffness by $50 \%[125,126]$. In the microscale ECM, these changes in material properties were kept consistent with the macroscale model, since it was extracted from a global ECM. For simplicity, the chondrocyte was assumed to be either living or dead regardless of the degenerative (OA) changes within the tissue. In this case, the living chondrocytes in the OA tissue had the same properties as the cells in healthy tissue, as the solid and fluid components within the cell are assumed to not change. For the PCM, few studies have shown changes in the PCM material properties with tissue depth in both healthy and OA cartilage using micropipette aspiration experimental technique [126], and atomic force microscopy [119]. From the first study, the ratios between these grades for the depthdependent E, and permeability was applied in this thesis to calculate the PCM material properties for OA tissue. The fibril stiffness $\xi$ for the PCM was reduced by $50 \%$ as done for the ECM $[125,126]$. The Poisson's ratio (v) for the PCM did not change significantly in each zone from healthy to OA tissues [126]. Table 4.1 summarizes the material properties used in this study for the ECM, PCM, and chondrocyte regions that were reported by experimental studies of degenerative (OA) cartilage. 
Table 4.1: Material properties employed in the ECM, PCM, and chondrocyte regions of biphasic fibril-reinforced OA cartilage microscale models.

\begin{tabular}{|c|c|c|c|c|c|c|c|}
\hline & \multicolumn{2}{|l|}{$\bar{E}(\mathrm{MPa})$} & \multicolumn{2}{|c|}{${ }^{1} \xi(\mathrm{MPa})$} & \multicolumn{2}{|c|}{$\kappa\left(\frac{m^{4}}{N * s}\right) * 10^{-15}$} & \multirow{2}{*}{$\begin{array}{l}\mathbf{v} \\
\text { All zones }\end{array}$} \\
\hline & $* \mathrm{SZ}$ & ${ }^{*} \mathrm{MZ} / \mathrm{DZ}$ & SZ & $\mathrm{MZ} / \mathrm{DZ}$ & SZ & $\mathrm{MZ} / \mathrm{DZ}$ & \\
\hline ECM & 0.71 & 0.71 & 0.6 & 0.6 & 4.6 & 4.6 & 0.03 \\
\hline PCM & 0.021 & 0.026 & 0.3 & 0.13 & 0.093 & 0.1125 & 0.03 \\
\hline Cell & $4.2 \times 10^{-4}$ & $2.4 \times 10^{-4}$ & - & - & 5 & 5 & 0.38 \\
\hline
\end{tabular}

${ }^{1}$ Fibril stiffness dominates primarily in tension (parallel to bearing surface) and is assumed negligible within the cell. ${ }^{*} \mathrm{SZ}, \mathrm{MZ}$, and DZ denote superficial, middle, and deep zones respectively.

As mentioned in Chapter 3, since the loading protocol was identical for both loading modes for the initial 2 seconds, the comparison between loading modes was made only after this time point, i.e. at approximately 100 seconds. The primary goal of this chapter was to compare the differences between loading modes in degenerative cartilage. Comparison of differences with healthy cartilage, including both loading modes will be discussed.

\subsection{Results}

The variables chosen to investigate the micromechanical environment of cartilage in this study were the $1^{\text {st }}$ and $3^{\text {rd }}$ principal strains, relative volume, fluid pressure, and fluid flux. The maximum solid shear stresses and strains were also reported for the chondrocytes but were not graphically shown as done with fluid flux. The numerical values for all variables studied in this thesis for OA cartilage under both loading modes are summarized in Appendix B. 


\subsubsection{Healthy vs. OA Cartilage Under Cyclic Sliding Indentation}

Principal Strains

Under cyclic sliding indentation, both the $1^{\text {st }}$ and $3^{\text {rd }}$ principal strains in OA cartilage followed similar trends as healthy cartilage described in Chapter 3, with differences in magnitudes. In all microscale models of OA cartilage under this loading mode, in the ECM, PCM, and chondrocyte regions, the $1^{\text {st }}$ principal strains were generally positive (Figure 4.1) and the $3^{\text {rd }}$ principal strains were negative (Figure 4.2). In both grades of cartilage under sliding loads, the peak averaged tensile and compressive strains showed minor differences between the central and peripheral locations, and with time, i.e. over 100 seconds. Similarly, in both tissues, the magnitudes and amplitudes of both tensile and compressive strains decreased with cartilage depth, i.e. from superficial to deep zone with strain amplifications in the chondrocyte region. 

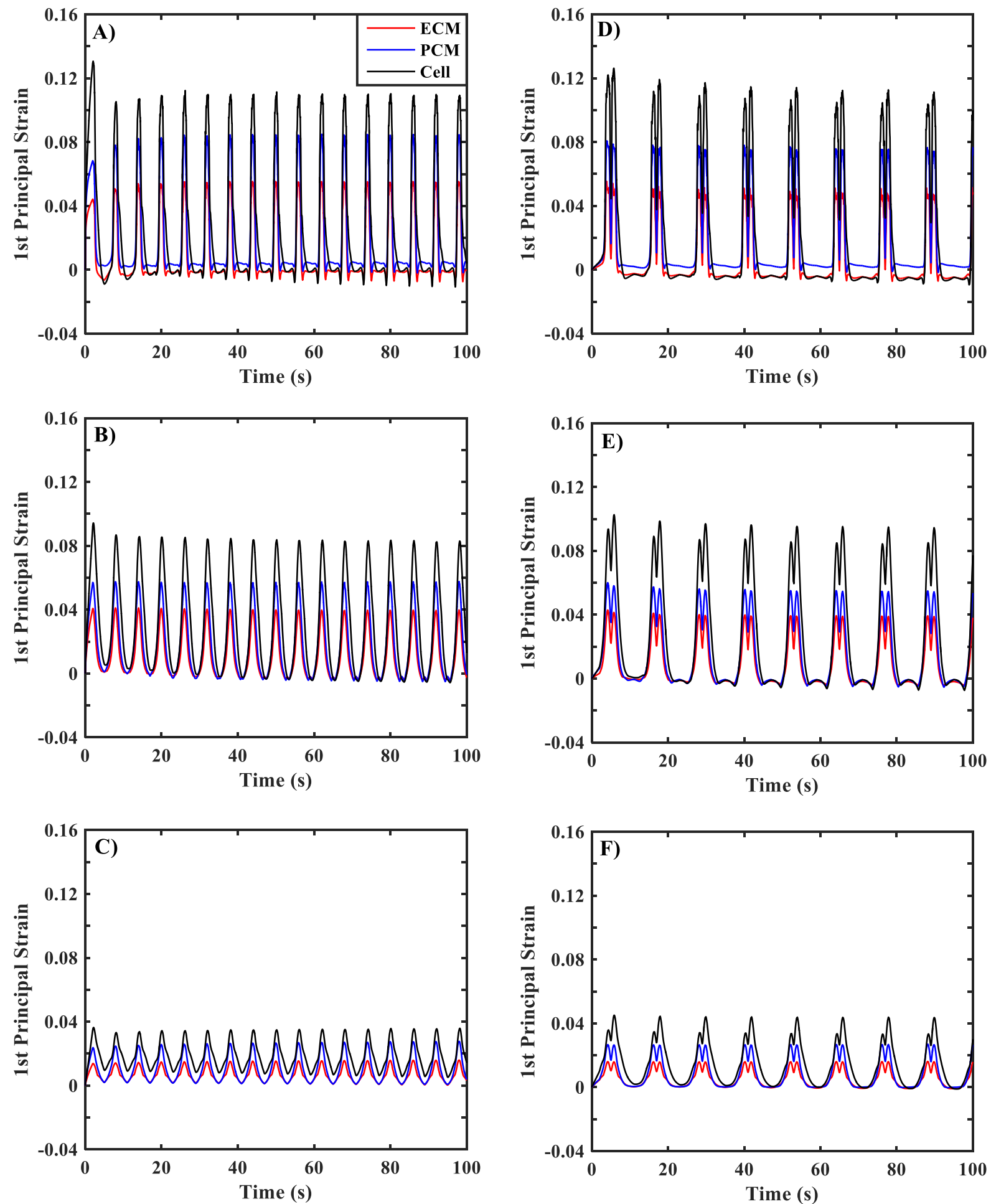

Figure 4.1: First principal strain in the microenvironment of OA cartilage at the superficial, middle, and deep zones of the central (A-C), and peripheral (D-F) locations under cyclic sliding indentation. 

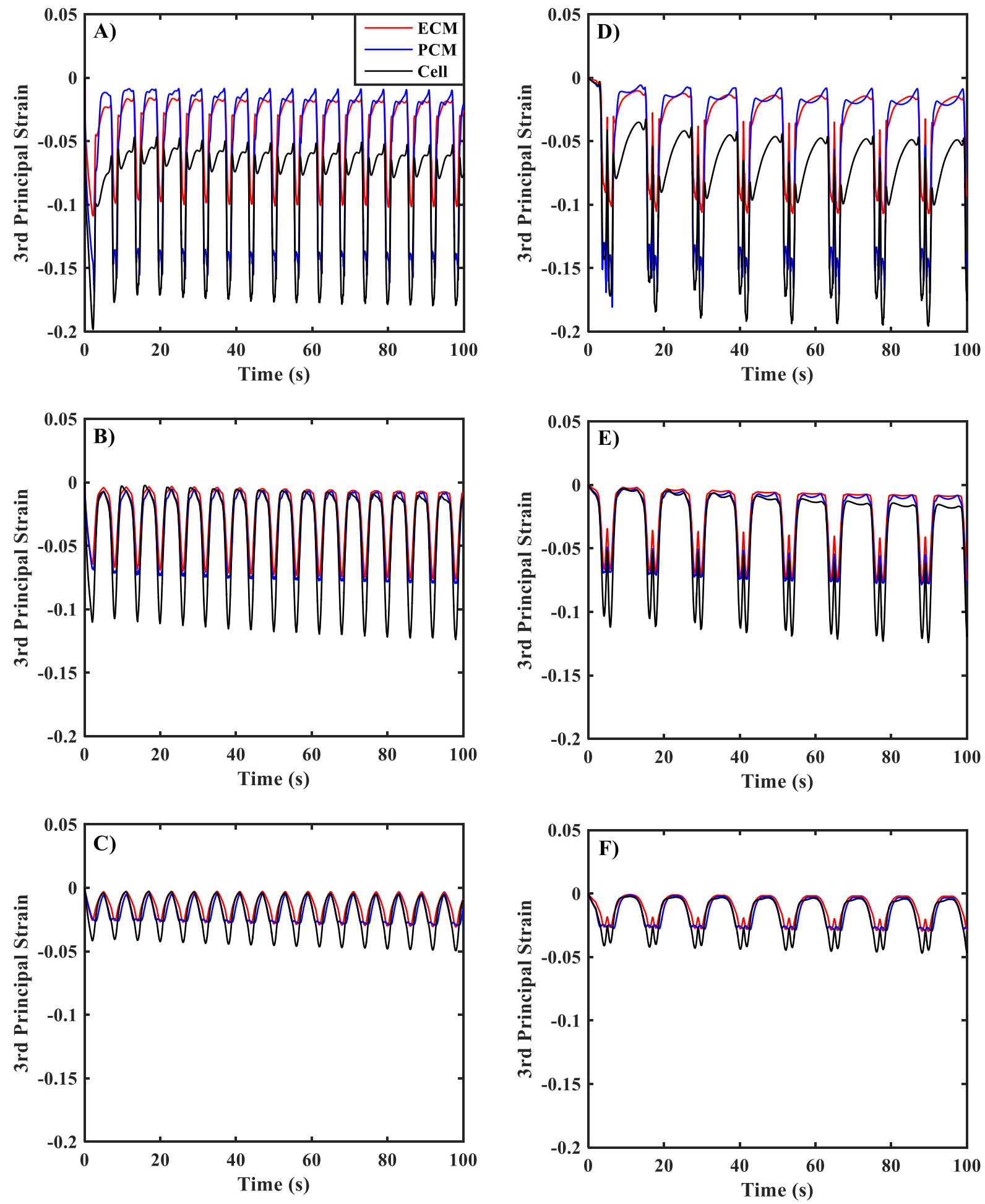

Figure 4.2: Third principal strain in the microenvironment of OA cartilage at the superficial, middle, and deep zones of the central (A-C), and peripheral (D-F) locations under cyclic sliding indentation. 
Figure 4.3 shows a comparison of the peak $1^{\text {st }}$ principal (tensile) strains between the microenvironment of healthy vs. OA cartilage under physiological cyclic sliding indentation. In all regions of all microscale models in OA cartilage, tensile strains were amplified relative to healthy cartilage. Over 100 seconds of sliding indentation, the peak averaged tensile strains in both grades of cartilage occurred in the chondrocytes of the superficial zone. At both central and peripheral locations of this zone, the tensile strains were approximately $10 \%$ for healthy and $12 \%$ for OA chondrocytes.

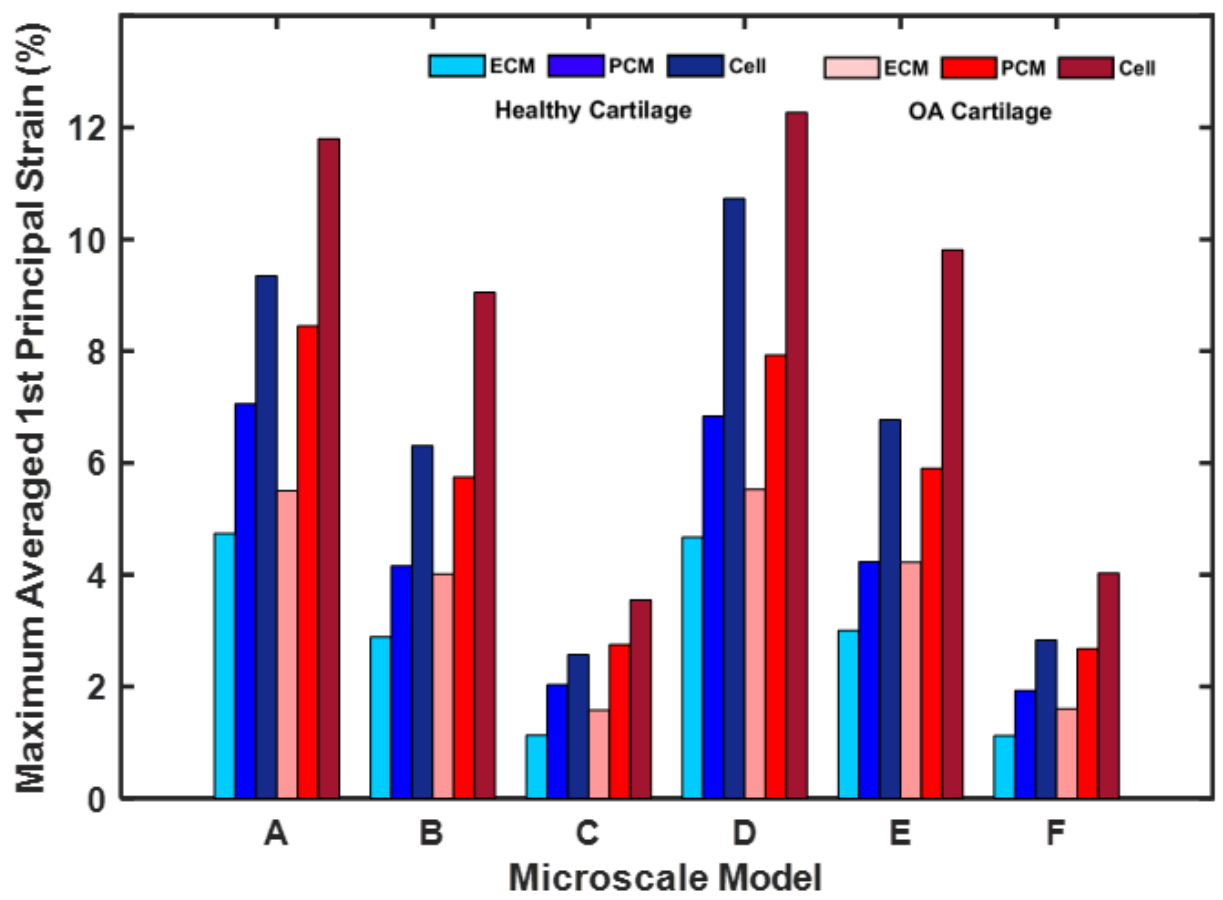

Figure 4.3: Maximum averaged first principal strain in the microenvironment of healthy vs. OA cartilage under cyclic sliding indentation.

Similarly, Figure 4.4 shows a comparison of the peak $3^{\text {rd }}$ principal (compressive) strains between the microenvironment of healthy and OA cartilage under this loading mode. Similar to the tensile strains, the compressive strains in OA were also increased in all regions of all microscale models compared to healthy cartilage. In both tissue grades 
under sliding loads, the compressive strains (Figure 4.4) were generally larger in magnitude compared to tensile strains (Figure 4.3) within each microscale model. Over 100 seconds of sliding indentation, the maximum averaged compressive strains in both grades of cartilage also occurred in the chondrocytes of the superficial zone. At both central and peripheral locations of this zone, the compressive strains were approximately $16.5 \%$ for healthy and $19 \%$ for OA chondrocytes.

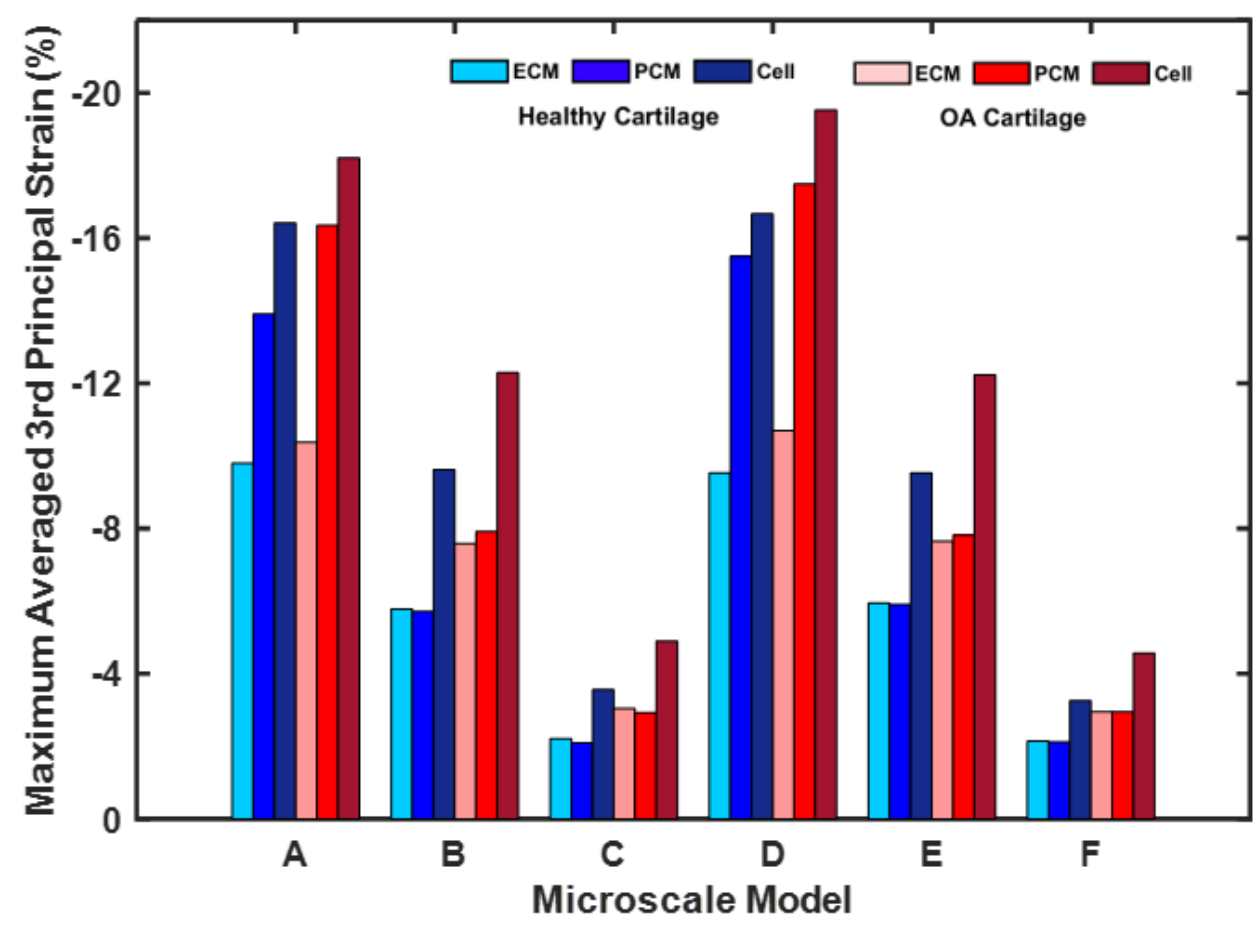

Figure 4.4: Maximum averaged third principal strain in the microenvironment of healthy vs. OA cartilage under cyclic sliding indentation.

\section{Relative Volume}

Under cyclic sliding indentation, the relative volume patterns and magnitudes in OA microscale cartilage models (Figure C.10) were almost identical to the microscale models in healthy cartilage (Figure C.3). For instance, in both cartilage grades, at the end 
of the initial ramp load ( 2 seconds), the largest increase in volume $(5 \%)$ occurred in the chondrocytes of the middle zone of both locations. Furthermore, in both tissue grades, the amplitudes generally decreased over time and with cartilage depth for both locations with largest amplitudes in the PCM regions of the superficial zone and chondrocyte regions of the middle and deep zones (Figure C.10). In comparison to results from Chapter 3, it can be concluded that there were minor changes in volume in all regions of all six locations studied with cartilage degeneration under sliding indentation.

\section{Fluid Pressure}

The general trends in fluid pressures in OA cartilage under cyclic sliding indentation (Figure 4.5) were very similar to those described for healthy cartilage in Chapter 3. As observed with the principal strains and relative volume, the amplitudes for fluid pressures also decreased with cartilage depth in both grades of tissue. Generally, the pressures were slightly higher at the peripheral location of the tissue. Major differences between healthy vs. OA cartilage under sliding indentation were between the pressure magnitudes. In Figure 4.6, it was found that fluid pressures decreased in all regions of the cartilage microenvironment with tissue degeneration. In healthy chondrocytes at the peripheral location, the pressures were approximately 235,44 , and $11 \mathrm{kPa}$ larger at the superficial, middle, and deep zones respectively compared to OA chondrocytes, which were comparably higher than at the central location. 

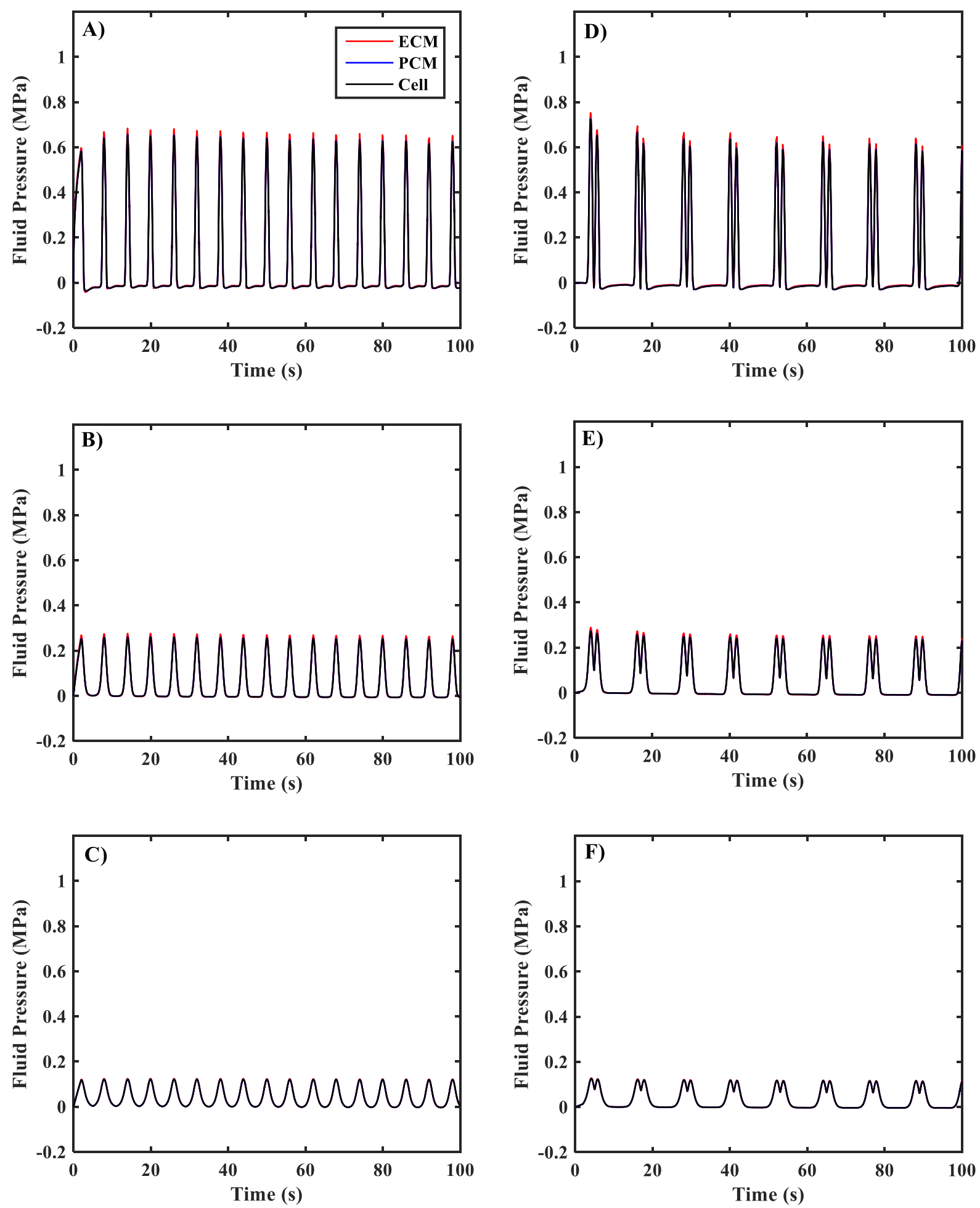

Figure 4.5: Fluid pressure in the microenvironment of OA cartilage at the superficial, middle, and deep zones of the central (A-C), and peripheral (D-F) locations under cyclic sliding indentation. 


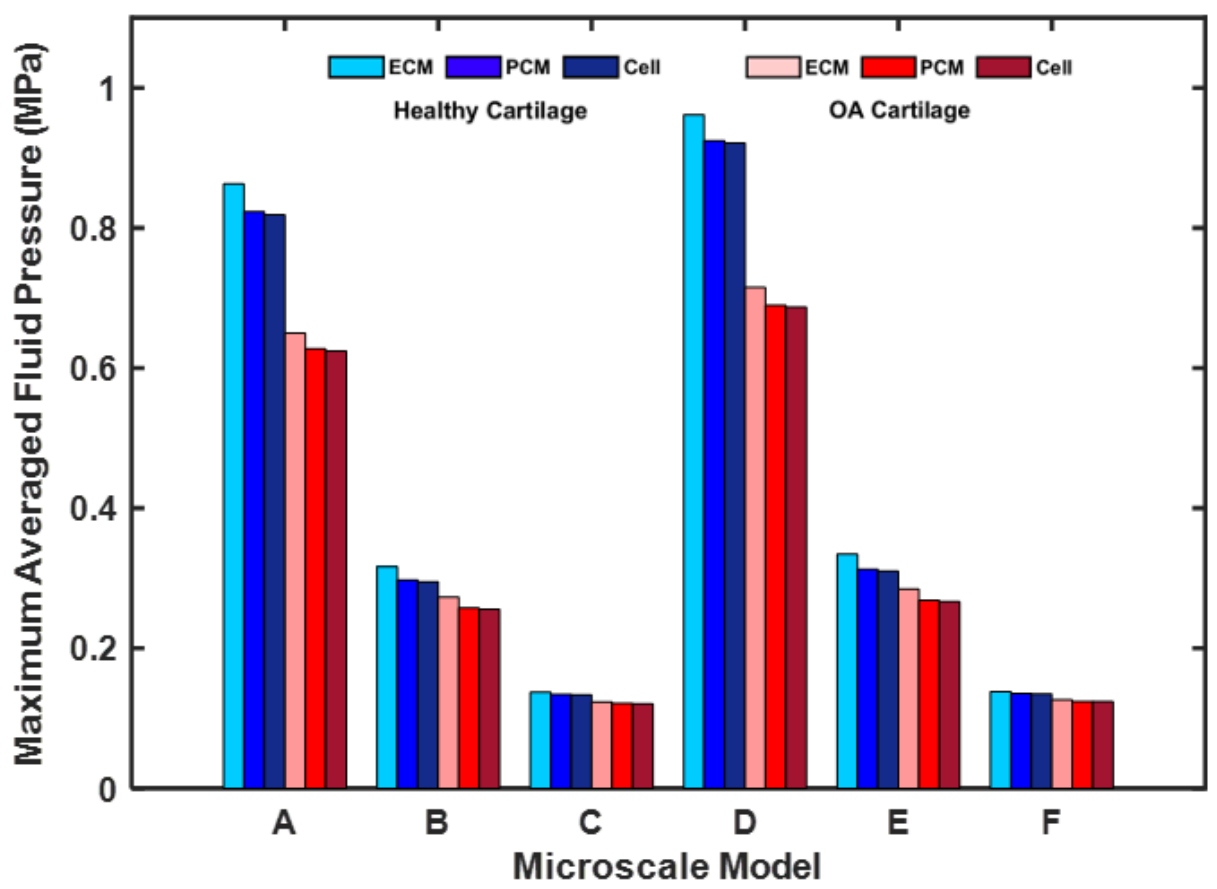

Figure 4.6: Maximum averaged fluid pressure in the microenvironment of healthy vs. OA cartilage under cyclic sliding indentation.

Fluid Flux

In both healthy and OA cartilage under cyclic sliding indentation. the peak fluid flux generally decreased with cartilage depth at both locations. The fluid flux in each region was non-uniform, with the flux in the ECM at least one order of magnitude higher compared to the chondrons. At this location, the ECM flux in healthy cartilage were 500, 200 , and $20 \mathrm{~nm} / \mathrm{s}$ higher in the superficial, middle, and deep zones respectively compared to OA cartilage. These differences were one order of magnitude smaller in each zone when comparing this ECM flux between loading modes in healthy cartilage. This suggests that the flux changes more significantly with loading modes than tissue grade. This trend was also true for the PCM and chondrocyte regions. Lastly, the direction of fluid flux did not change significantly between healthy vs. OA cartilage under cyclic 
sliding indentation. For a detailed description of flux directions under this loading mode, refer to Figure C.7 in Appendix C.

\section{Maximum Chondrocyte Shear Stresses and Strains}

In both tissues, the shear stresses and strains decreased with cartilage depth at both locations. However, both maximum shear stresses and strains were amplified in OA cartilage compared to healthy cartilage under sliding indentation. The largest shear strains in healthy and OA chondrocytes were approximately $13 \%$ and $15 \%$ respectively in the superficial zone of both locations (Figure 4.7). The differences between tissue grades were smaller relative to the differences between sliding and axial quasi-static indentation loading modes. This finding suggests that applying a non-physiological loading mode may result in differences in shear strains that are more significant than degenerative changes.

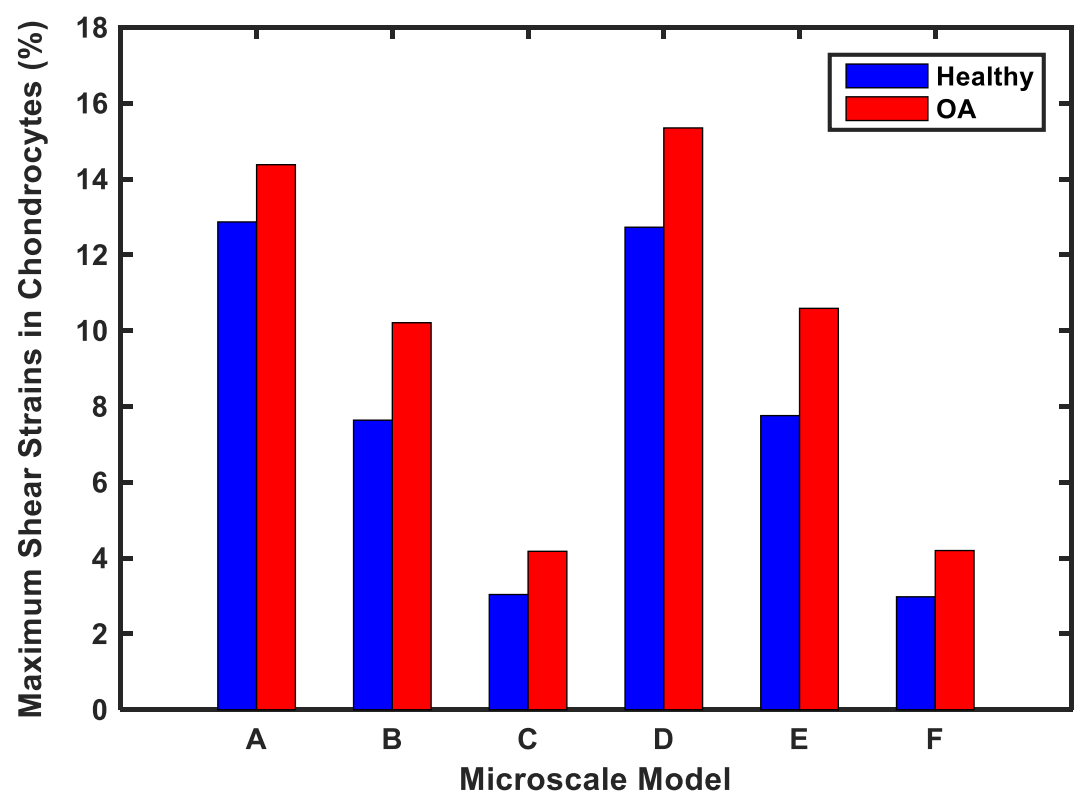

Figure 4.7: Maximum shear strains in healthy vs. OA chondrocytes under cyclic sliding indentation. 


\subsection{Discussion}

Similar to healthy cartilage in Chapter 3, the micromechanical environment of OA cartilage was investigated for six different locations under the novel physiological cyclic sliding conditions. The more common axial quasi-static indentation was also investigated for selected variables to compare with physiological sliding loads. This was achieved using BPVE fibril-reinforced multiscale finite element models with depth-dependent material properties and shape of chondrons. The goal was not only to assess the influence

of cartilage degeneration under physiological sliding loads but also to evaluate whether or not these differences may be affected by a less physiological axial quasi-static indentation. In both healthy and OA cartilage, it was found that both loading modes and spatial locations had an impact on the principal strains, and fluid pressures and fluxes in the ECM, PCM, and chondrocyte regions.

\subsubsection{Healthy vs. OA Cartilage Under Cyclic Sliding Indentation}

\section{Principal and Shear Strains}

In both healthy and OA cartilage under cyclic sliding indentation, the magnitudes, and amplitudes for the $1^{\text {st }}$ principal strains (Figures 3.10 and 4.1 ) and $3^{\text {rd }}$ principal strains (Figures 3.13 and 4.2) decreased with cartilage depth. The increased deformations at the cartilage surface were expected since the location of the microscale models in the superficial zone were closer to the applied macroscale load than the middle and deep zones. However, within each microscale model, the magnitude and amplitude differences for both principal strains were minor over time, i.e. over 100 seconds, and with locations, i.e. central vs. peripheral. The former was due to the loaded and unloaded cycles that resulted in small consolidation, i.e. strain, over time since the tissue was able to re- 
hydrate during the unloaded portion of the cycle. The latter was expected as the sliding motion of the indenter provides local stimulation at both the tissue center and periphery. Furthermore, in both grades of cartilage, strain amplifications were found in the chondrocytes since they were two orders of magnitude softer than the PCM and four orders of magnitude softer than the ECM. With cartilage degeneration under cyclic sliding indentation, the most tensile (Figure 4.3) and most compressive (Figure 4.4) principal strains increased in all regions of all zones at both locations. This was also true for maximum intracellular shear strains (Figure 4.8). These findings were expected due to the reduction in aggrecan and fibril stiffness in OA cartilage which simulates PG depletion $[41,125]$ and collagen fibrillation $[125,126]$. Therefore, OA cartilage would be subject to less compressive and tensile resistance than healthy cartilage, resulting in larger deformations.

\section{Fluid Pressure and Flux}

In each cartilage grade, the general trends, i.e. depth- and time-dependent changes in fluid pressures (Figure 4.5) were similar to the principal strains described above. The main difference was that fluid pressures decreased with cartilage degeneration (Figure 4.6). This was to be expected due to the $20 \%$ increase in the permeability [41, 125], causing the fluid to flow easily and faster from the loaded region, and therefore resulting in low pressurization. Between these cartilage grades, the largest difference was approximately $200 \mathrm{kPa}$ at both locations of the superficial zone, whereas it was less than $50 \mathrm{kPa}$ for the middle and deep zones. This may suggest that the fluid microenvironment with cartilage degeneration is more significant at the surface. Unlike fluid pressures, both 
magnitudes and direction for the fluid flux in the cartilage microenvironment did not change significantly with degeneration.

\subsubsection{OA Cartilage Under Various Loads}

As observed with healthy cartilage in Chapter 3, the differences between the more physiological cyclic sliding indentation vs. axial quasi-static indentation were also very similar in OA cartilage. Between healthy and OA cartilage, the differences occurred primarily in magnitudes due to the change in material properties as discussed above. However, between the loading modes, the differences were mainly time- and locationdependent due to variation in loading protocols. For instance, the $1^{\text {st }}$ and $3^{\text {rd }}$ principal strains and fluid pressures under cyclic sliding indentation showed minor differences at a particular zone for both central and peripheral locations. However, under axial quasistatic indentation, major differences were found between these locations. Under cyclic sliding indentation, this was due to the constant sliding motion of the indenter causing both the cartilage center and periphery to be stimulated. On the other hand, in axial quasistatic indentation, the fixed centered position of the indenter resulted in relatively larger magnitudes at the cartilage center.

As observed with healthy cartilage, in OA cartilage, the most tensile $1^{\text {st }}$ principal strains and the fluid pressures were lower for all regions of all microscale models under axial quasi-static indentation compared to sliding indentation. The most compressive $3^{\text {rd }}$ principal strains were higher for all regions of all zones at the central location but lower for at the peripheral location. The former is due to the transient nature of cyclic sliding indentation with minimal consolidation allowing the tissue to recover at the unloaded phases. This was also true for fluid pressurization under sliding loads. However, for $3^{\text {rd }}$ 
principal strains in axial quasi-static indentation, the central microscale models underwent larger compression compared to sliding indentation, since the indenter was stationary at the central location applying a constant load over 100 seconds. With this logic, the relatively smaller compressive strains found in the peripheral microscale models under axial quasi-static indentation were reasonable since the peripheral models were not directly loaded by the indenter.

\subsubsection{Differences Between Healthy vs. OA Cartilage Under Various Loads}

As discussed in previous sections and Chapter 3, differences existed between cartilage grades and between loading modes. Figure 4.8 shows the absolute differences in the $1^{\text {st }}$ principal (most tensile) strains between healthy and OA cartilage under cyclic sliding and axial quasi-static indentation. The strains shown are absolute differences between cartilage grades (OA-healthy) converted into percentages. Under sliding indentation, the $1^{\text {st }}$ principal strains were larger in all OA microscale models, as expected, hence the positive differences (Figure 4.8). However, under axial quasi-static indentation, these differences were negative in all regions of microscale model D and the ECM region of microscale model A (Figure 4.8). Furthermore, under sliding indentation, out of all zones, the maximum difference in the $1^{\text {st }}$ principal strains between cartilage grades was highest in the middle zone and largest (2.75\%) in the chondrocyte region (Figure 4.8). This was not entirely true under axial quasi-static indentation, as microscale models A and D were exceptions. 


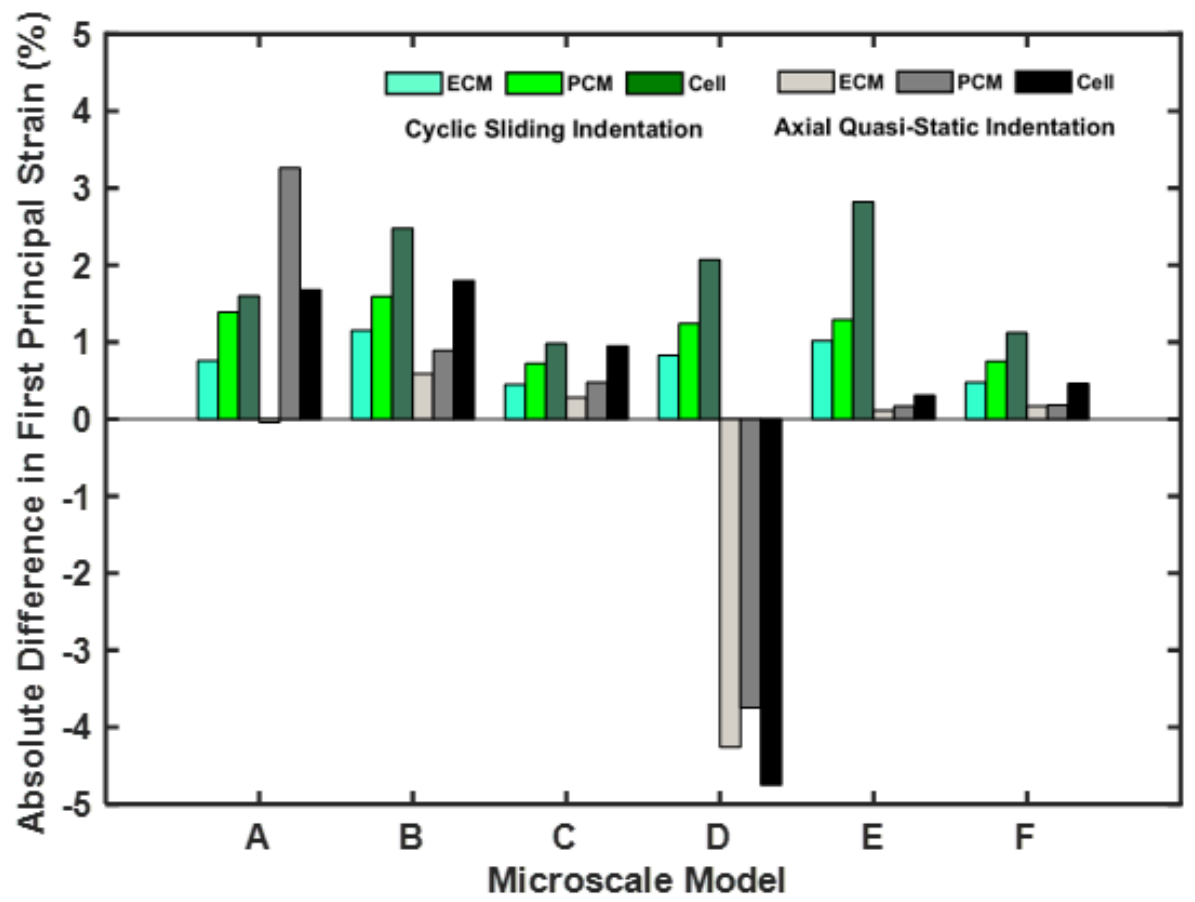

Figure 4.8: Absolute differences in the first principal strains of [OA-healthy] cartilage microenvironment under cyclic sliding vs. axial quasi-static indentation.

Similarly, the percent differences in the fluid pressures between the healthy and OA cartilage are shown under cyclic sliding and axial quasi-static indentation (Figure 4.9), which were calculated with respect to the healthy values. For all microscale models, the percent differences in fluid pressure were negative, i.e. decreased due to cartilage degeneration and were larger under cyclic sliding than under axial quasi-static indentation (Figure 4.9). The largest percent difference was approximately $25 \%$ at both locations of the superficial zone under cyclic sliding indentation. Furthermore, the pressure decreases generally decreased with cartilage depth under both loading modes, but differences were higher under cyclic sliding indentation (Figure 4.9). It can be concluded that both principal strains and fluid pressures can be wrongfully predicted if a less physiological axial quasi-static indentation is applied. 


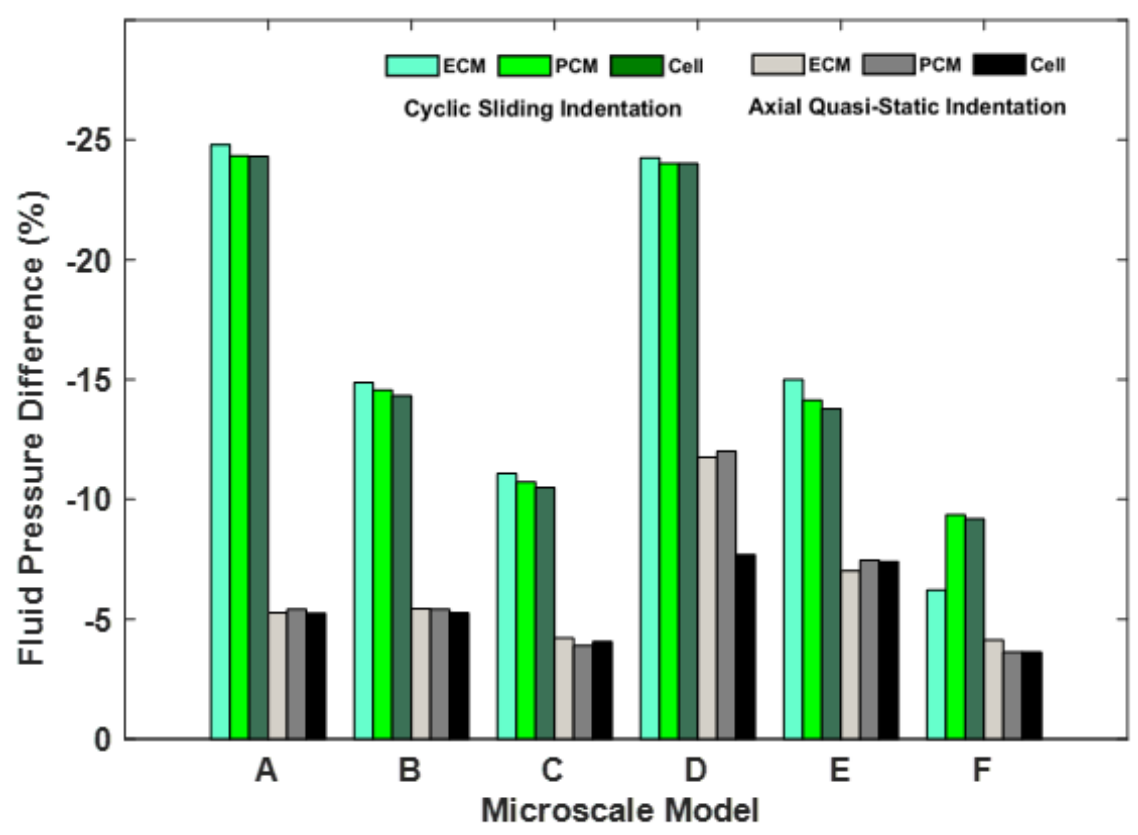

Figure 4.9: Percent differences in the fluid pressure with cartilage degeneration under cyclic sliding vs. axial quasistatic indentation. 


\section{Overall Discussion}

In Chapter 3, the design, methodology, and analysis of healthy BPVE fibrilreinforced cartilage microscale models were presented. In Chapter 4, these microscale models were varied with degenerative (OA) properties under the same loading conditions. The ECM of each microscale model was designed as a 3D cube with the PCM and chondrocyte regions embedded at the centroid. In the past, many studies have modelled the microenvironment in 2D [71, 76] and more recently in 3D [77], however, these studies have not considered the same variables as done in this thesis. For instance, for the first time, these 3D microscale models implemented collagen fibril-reinforcement as they were mainly incorporated in 2D axisymmetric models in the past $[36,76,89]$. These 3D microscale models allow for a more realistic representation of the collagen fiber structure and distribution [102]. Furthermore, the axisymmetric assumption does not allow for the implementation of sliding indentation since loads are not axisymmetric. Therefore, for the first time, both axial quasi-static and cyclic sliding indentation loading modes were incorporated to study the multiscale mechanical behaviour of cartilage. Sliding indentation was an important type of loading to consider since it better mimics the physiological loading condition compared to a more commonly employed unconfined compression [100], and it has recently been recognized as an important loading mode $[99,100,127]$. The migrating contact area in indentation locally deforms the cartilage surface as opposed to the global stimulation provided in unconfined compression [100]. This thesis also investigated the influence of the depth-dependent shape of chondrocytes, which to date, has only been studied with 2D axisymmetric microscale models in unconfined compression without fibril-reinforcement [96]. For the first time, a microscale 
sub-model in each of the superficial, middle, and deep zones at both the central and peripheral locations were developed resulting in six novel FE models. In the past, these spatial locations of the microscale cartilage models were only considered in $2 \mathrm{D}$ in unconfined static compression [71]. Furthermore, the depth-dependent mechanical properties in the PCM and chondrocyte regions in each microscale model were implemented to represent both healthy and degenerated (OA) cartilage.

This thesis investigated the biomechanical behaviour of the cartilage microenvironment using 3D BPVE fibril-reinforced FE micromodels. However, many simplifying assumptions were made both at the macro- and microscale models. In terms of geometry, the diameter of the indenter was small and thus the contact area was smaller relative to the typical joint in-vivo $[115,116]$. Additionally, the 0.5 Newton force applied at the macroscale was also significantly lower than physiological loads to avoid solver issues. However, the combination of lower indenter diameter and sliding speeds, and uniaxial force was able to reproduce physiological contact stresses, based on experimental studies $[115,116]$. Also, the material properties applied at the macroscale, i.e. the ECM region, were assumed in both healthy (Table 3.2) and OA (Table 4.1) from previous experimental studies $[39,41,118.125]$. The properties in OA were more heterogeneous with extensive PG depletion and therefore the ECM region in healthy cartilage was modelled as a homogeneous mixture throughout the cartilage depth. Also, in both tissues, all regions of the tissue were assumed to be biphasic by neglecting swelling properties.

At the microscale level, the assumption of a single chondron at the centroid of each sub-model was made due to their scarce population within the macroscale cartilage. 
Furthermore, to date, this was the first study to implement the chondrocyte depthdependent geometry in 3D. Therefore, the $3^{\text {rd }}$ dimension along the $y$-axis was assumed to be the same as the major axis of the chondrocyte in the corresponding zone. Even the xand z-axes obtained from a previous numerical study [96] were merely an approximation. The material properties applied at the microscale, i.e. the PCM and chondrocyte regions, were assumed from experimental studies including micropipette aspiration and atomic force microscopy techniques $[71,119,120,121,126]$.

There were some limitations associated with the autonomous post-processing approach implemented in this thesis. In determining the boundary conditions for all surface nodes on all microscale models, linear interpolation functions given in equations 11-14, since the previously modelled macroscale tissue made use of linear hexahedral elements. Also, this was a simplified assumption made at the microscale, since there were 24 models studied in this thesis each with 392 boundary conditions prescribed. Furthermore, the simulation time for all analyses was evaluated over 100 seconds to ensure the completion of 8 complete cycles in sliding indentation. Since this time limit was also imposed under axial quasi-static indentation for consistency, it was not clear whether steady state was achieved at 100 seconds. In a study using static unconfined compression, this equilibrium state was achieved at approximately 3000 seconds [71].

The ECM, PCM, and chondrocyte regions studied in this thesis were based on spatial nodes chosen along the axis of compression, as shown in Figure 3.7. Furthermore, the central node was chosen for the chondrocyte region in all microscale models as done in past studies [71], and based on the assumption that this is the core of the cell containing the nucleus, a major organelle that regulates protein synthesis, metabolism, 
and reproduction. Also, the intracellular shear stresses and strains presented in this thesis were for the solid phase and this was chosen since these have been correlated with degenerative changes $[74,80]$. It also has reported that fluid shear stress alters chondrocyte metabolism $[73,75]$ and may not be negligible under dynamic loads due to larger transient flows [71]. Lastly, there was no experimental validation provided for the microscale models as this is challenging with methods currently available.

\subsection{Cartilage Mechanobiology}

The findings from this thesis can be linked to current in-silico and in-vitro studies on cartilage mechanobiology to study their potential influence on modulating the chondrocyte response in-vivo. As mentioned in Section 1.4.4.3, articular cartilage is subjected to a range of mainly compressive static and dynamic loads with varying frequencies and magnitudes [71]. The peak stresses can be as high as $20 \mathrm{MPa}$, and compressive strains may reach up to $45 \%$ [57].

\section{Dynamic Loads}

Generally, cyclic dynamic compression can promote matrix synthesis, however, it is highly dependent on the magnitude and frequency of the applied load [22]. For instance, Kim et al. showed that in cartilage from calf knee joints, the synthesis of aggrecan and other proteins was invariable for load magnitudes less than $5 \%$ at frequencies less than $0.001 \mathrm{~Hz}$ [59]. However, low magnitude compression with higher frequencies $(0.01-1 \mathrm{~Hz})$ increased PG and protein synthesis by $40 \%$ [59]. In this thesis, the magnitude of axial compression was $5 \%$ and the frequency was about $0.08 \mathrm{~Hz}$, both which are within the range to stimulate matrix synthesis. The strain rate is also a contributing factor in regulating matrix synthesis, as Kurz et al., showed that in cartilage 
explants, a $50 \%$ strain applied at a rate of $0.1-1 \mathrm{~Hz}$, resulted in a substantial decrease in matrix synthesis and increased chondrocyte death [72]. However, the cells that happened to survive this strain rate were unable to respond normally under low magnitude cyclic compression [72].

More recently, the novel physiological sliding indentation loading condition has been used to study cartilage mechanobiology $[99,100,127]$. An experimental study showed that bovine chondrocytes seeded in an agarose-sandwich model under sliding indentation at $10 \%$ depth and $1 \mathrm{~Hz}$, resulted in an increased amount of collagen [100]. Another numerical study showed that with the addition of lateral compression-sliding indentation, the perpendicular orientation of the collagen fibers found in the deep zone of cartilage may be stimulated [99]. Furthermore, with increased duration of sliding motion, both the depth-dependent gene and protein expression levels increased, with amplified responses at regions with larger strains [100]. In this thesis, large principal strains were found at central and peripheral locations of the superficial zone, suggesting a more active metabolic response at the cartilage surface. Another recent in-vitro study correlated mechanical parameters to the expression of different genes and proteins by using a multiple linear regression model [127]. It was shown that sliding speeds between 10-70 $\mathrm{mm} / \mathrm{s}$ with a normal force of $100 \mathrm{~N}$ applied to cartilage explants showed higher expression of lubricin and ADAMTS-5 [127]. The former is vital for cartilage lubrication and the latter is associated with the breakdown of aggrecan [5]. This is relevant as the physiological contact stresses found in their study was approximately $1 \mathrm{MPa}$, which was also achieved in this study with a lower force $(0.5 \mathrm{~N})$ and sliding speed $(1 \mathrm{~mm} / \mathrm{s})$. Along with deformation, the spatial profile of interstitial fluid flow has shown to be related to 
the synthesis of aggrecan, mainly in the middle zone [79]. Finite element analysis has shown that sliding indentation resulted in a distributed spatial pattern of fluid flow which also vary with sliding speeds [128]. This flow may also be important for the proper transport of nutrients across different regions of the tissue [77].

This thesis also investigated the intracellular shear stresses and strains. In previous in-vitro studies, shear loading devices have shown that dynamic shear strain of $1 \%$ applied at $0.1 \mathrm{~Hz}$ for 24 hours has shown to increase protein and PG synthesis by $25 \%$ and $41 \%$ respectively [57]. Furthermore, isolated human chondrocytes subjected to large shear strains caused an increase in GAG synthesis and expression of IL-6 [73]. Although IL's are generally catabolic cytokines, IL-6, in particular, has shown to reduce the destruction of cartilage in IL-6-deficient mice in experimental arthritis [129]. In this thesis, under axial quasi-static indentation, all zones of the central chondrocytes had significantly larger shear strains and stresses than under sliding indentation but were lower at the peripheral location. Furthermore, all OA chondrocytes had increased shear stresses and strains relative to healthy chondrocytes. High levels of intracellular shear stresses have been correlated to the synthesis of $\mathrm{NO}[74,75]$, which is a biological marker of OA.

Static Loads

Static compression can inhibit matrix synthesis depending on the magnitude and duration of the applied load. It has been shown that $15 \%$ static compression is enough to inhibit matrix synthesis significantly [59]. In-vitro static equilibrium compression of cartilage has shown to decrease of aggrecan synthesis and is linked to the structure of the chondrocyte and nucleus [130]. The decreased synthesis of aggrecan was found to be 
proportional to the applied stress in both static and dynamic compression [60]. However, it was predicted that decreased metabolism may be dominated by the static component of the dynamic load [60]. A study on bovine cartilage under static unconfined compression showed that in cyclic static compression, the protein synthesis increased by approximately $50 \%$, but PG synthesis was inhibited due to progressive consolidation [131]. In a similar study on bovine cartilage, aggrecan and collagen type-II mRNA expression decreased with longer periods of static compression [132]. Long durations of static compression were also associated with fibronectin, which is a catabolic protein that marks the breakdown of cartilage ECM [131]. In this thesis, this is relatable to the central microscale models under axial quasi-static indentation, especially at the superficial zone, where they experienced the largest $3^{\text {rd }}$ principal (most compressive) strains due to constant compression applied over 100 seconds. Lastly, an in-vitro study showed that transplanted bovine chondrocytes loaded under confined static compression of $0.48 \mathrm{MPa}$ for 16 hours resulted in $41 \%$ larger PG synthesis after load removal than unloaded control chondrocytes [133]. This may suggest lower PG synthesis for peripheral microscale models, especially in the deep zone, since they were not directly stimulated by the indenter resulting in little to no deformation.

\subsection{Future Work}

The modelling technique implemented can be extended to larger geometries and forces that match in-vivo physiological conditions of a joint. However, practically, the higher force leads to ploughing of the indenter through the tissue which can lead to solver issues. With an experimental setup, the specimen size is relatively small to avoid large surface curvature. Also, the ECM materials in this biphasic study can include depth- 
dependent properties in the future as these have been previously predicted for the aggregate modulus and permeability [109, 134]. Moreover, the inclusion of swelling properties can be considered in a more comprehensive triphasic material model. Recently, it was found that there were increased mean axial strains and decreased fibril strains with a decrease in fixed charge density of the PGs in the knee joint cartilage during simulated gait cycle [135]. Furthermore, these effects were amplified with collagen fibrillation found in OA [135]. In the microscale level, another study found that the depth-dependent fixed charge densities in the ECM, PCM, and chondrocyte regions primarily influenced the aspect ratios and the solid stresses of the cell [36].

In the future, at the microscale level, it would be interesting to investigate the effect of stress/strain shielding due to the existence of multiple chondrons within each microscale model. Although this could be implemented in both cartilage grades, it has been reported that isogenous clusters of chondrocytes are formed especially in the deeper zones of OA cartilage [52]. A more comprehensive study may include the anatomical composition and distribution of cartilage components based on histological images shown in Figure 1.4.

In the future, the simulation time for all analyses could be extended to over 100 seconds to examine when the state of equilibrium is achieved in creep loading. Moreover, the results presented for the ECM, PCM, and chondrocyte regions should also consider nodes along the radial axis in the future. Different nodal locations other than the central node of the chondrocyte should be investigated in the future as the maximum intracellular does not necessarily occur at this location for all time points. The intracellular shear stresses and strains should be investigated for the fluid microenvironment as it has been 
reported that fluid shear stress alters chondrocyte metabolism $[73,75]$, and can be estimated with the fluid viscosity, velocity, and permeability [136].

In this study, a 5\% uniaxial compression was applied at the macroscale cartilage with an indenter that was either static or sliding over the tissue for 100 seconds. In the future, different loading protocols that mimic other daily activities such as running, stairclimbing, and even stumbling can be incorporated to predict the behaviour of the chondrocytes and their immediate microenvironment. These computational studies may be useful in combination with existing experimental studies on mechanobiology for detecting early stages of cartilage degeneration and improving material properties of tissue-engineered cartilage. At present, as an extension of this study, an experimental apparatus was developed to test cartilage specimens under creep to determine best-fit biomechanical properties from the literature using an optimization routine in FEBio2.6. Similarly, these specimens were loaded with physiological sliding to estimate the fluid load support in articular cartilage. Currently, these experimental results are unpublished, but can be used to validate the biomechanical response of microscale cartilage obtained in this study. 


\section{Conclusions}

\subsection{Thesis Summary}

This thesis examined the influence of physiological mechanical loading on the biomechanical behaviour of the microenvironment of six 3D BPVE fibril-reinforced cartilage models. This was achieved by implementing a multiscale autonomous postprocessing approach that made use of FEA to couple the macro- and microscopic scales. The local microenvironment of each microscale model was designed to incorporate a single chondrocyte within the PCM both of which were embedded at the centroid of a cubic volume representing the local ECM. All six microscale models were tested under the novel and physiological cyclic sliding indentation and the more common axial quasistatic indentation for healthy (Chapter 3) and OA (Chapter 4) cartilage, resulting in 24 distinct microscale models. All analyses were performed in FEBio2.5.2 open-source software with the consideration of influential variables that either have not been brought to light or studied in great detail in present studies on cartilage mechanobiology. These may be useful in determining how the chondrocyte response and the overall tissue metabolism is modulated in-vivo.

\section{The microenvironment of healthy vs. OA cartilage under cyclic sliding indentation}

In both healthy and OA cartilage under cyclic sliding indentation, the magnitudes, and amplitudes for the $1^{\text {st }}$ principal strains (Figures 3.10 and 4.1 ), $3^{\text {rd }}$ principal strains (Figures 3.13 and 4.2), and fluid pressures (Figure 3.18 and 4.6) decreased with cartilage depth. Within each microscale model, these changes were minor over time, i.e. over 100 seconds, and with locations, i.e. central vs. peripheral. 
With cartilage degeneration under cyclic sliding indentation, the most tensile (Figure 4.3), most compressive (Figure 4.4) principal strains, and maximum shear strains (Figure 4.8) increased in all regions of all zones with strain amplifications in the chondrocytes. These were largest at the superficial zone chondrocytes at both locations of both healthy and OA cartilage and were reported to be $10 \%$ and $12 \%$ for the $1^{\text {st }}$ principal and $-16.5 \%$ and $-19 \%$ for the $3^{\text {rd }}$ principal strains respectively.

Whereas, fluid pressure decreased with cartilage degeneration in all regions of all zones at both locations due to increased permeability. Unlike fluid pressures, both magnitudes and direction for the fluid flux in the cartilage microenvironment did not change significantly with cartilage degeneration.

\section{The microenvironment of $\mathrm{OA}$ cartilage under different loading modes}

In both healthy and $\mathrm{OA}$ cartilage, the magnitudes of the $1^{\text {st }}$ and $3^{\text {rd }}$ principal strains and fluid pressures under the less physiological axial quasi-static indentation showed major differences, i.e. were generally larger at the central location relative to the peripheral location in all zones relative to sliding indentation. Relative to sliding indentation, under axial quasi-static indentation. the $3^{\text {rd }}$ principal (most compressive) strains were approximately overestimated by $25 \%$ at the central and underestimated by $20 \%$ at the peripheral locations at the superficial zone of both healthy and OA cartilage. Whereas, the $1^{\text {st }}$ principal strains and fluid pressure were lower in all regions of all zones under axial quasi-static compared to cyclic sliding indentation.

Differences between healthy and OA cartilage under different loading modes 
Under the physiological cyclic sliding indentation, the absolute differences in the $1^{\text {st }}$ principal strains between cartilage grades (OA-healthy) were positive (Figure 4.9), implying the strain magnitudes increased with cartilage degeneration. Whereas, under axial quasi-static indentation, these differences were unexpectedly negative in all regions of microscale model D and the ECM region of microscale model A (Figure 4.9). Similarly, for all microscale models, the percent differences in fluid pressure were negative, which implied that the pressure decreased due to cartilage degeneration (Figure 4.10). Furthermore, percent differences were larger under cyclic sliding than under axial quasi-static indentation (Figure 4.10), but in both cases decreased with cartilage depth. These differences implied that wrong predictions may be made for principal strains and fluid pressures when the less physiological static indentation is applied.

\subsection{Thesis Contributions}

The major contributions of this thesis are listed as follows;

1. Developed six novel BPVE fibril-reinforced microscale models of cartilage to test under cyclic sliding and axial quasi-static indentation using a multiscale autonomous post-processing approach.

2. Analyzed the biomechanical microenvironment, i.e. the ECM, PCM, and chondrocyte regions at the superficial, middle, and deep zones of the central and peripheral locations of healthy and OA cartilage.

3. Wrong conclusions were made about cartilage degeneration with non-physiological axial quasi-static indentation.

4. Modelling technique employed can be used in mechanobiology studies to better understand in-vivo mechanoregulation of chondrocytes. 


\section{References}

[1] A. Litwic, M. H. Edwards, E. M. Dennison, and C. Cooper, "Epidemiology and burden of osteoarthritis," British Medical Bulletin, vol. 105, no. 1, pp. 185-199, 2013.

[2] K. V. MacDonald, C. Sanmartin, K. Langlois, and D. A. Marshall, "Symptom onset, diagnosis and management of osteoarthritis.," Health reports, vol. 25, no. 9, pp. 10-7, 2014.

[3] K. Wilkins, "Incident arthritis in relation to excess weight.," Health reports, vol. 15, no. 1, pp. 39-49, 2004.

[4] Arthritis Alliance of Canada, "The impact of arthritis in Canada: today and over the next 30 years," Arthritis Alliance of Canada, vol. Fall, no. 3, p. 52, 2011.

[5] R. F. Loeser, S. R. Goldring, C. R. Scanzello, and M. B. Goldring, "Osteoarthritis: A disease of the joint as an organ," Arthritis \& Rheumatism, vol. 64, no. 6, pp. 1697-1707, 2012.

[6] J. S. Khurana, "Bone Pathology," in Bone Pathology, 2nd ed., F. F. S. M.Barbe, J.B. Driban, S.N. Popoff, A.E. Barr, Ed. New Jersey: Humana Press, 2009, pp. 51-60.

[7] G. W. Greene, X. Banquy, D. W. Lee, D. D. Lowrey, J. Yu, and J. N. Israelachvili, "Adaptive mechanically controlled lubrication mechanism found in articular joints," Proceedings of the National Academy of Sciences, vol. 108, no. 13, pp. 5255-5259, 2011.

[8] A. J. Sophia Fox, A. Bedi, and S. A. Rodeo, "The basic science of articular cartilage: Structure, composition, and function," Sports Health, vol. 1, no. 6, pp. 461-468, 2009.

[9] V. C. Mow, A. Ratcliffe, and A. Robin Poole, "Cartilage and diarthrodial joints as paradigms for hierarchical materials and structures," Biomaterials, vol. 13, no. 2, pp. 6797, Jan. 1992.

[10] T. J. Lyons, R. W. Stoddart, S. F. McClure, and J. McClure, "The tidemark of the chondro-osseous junction of the normal human knee joint," Journal of Molecular Histology, vol. 36, no. 3, pp. 207-215, 2005.

[11] M. Pacifici, E. B. Golden, O. Oshima, I. M. Shapiro, P. S. Leboy, and S. L. Adams, "Hypertrophic Chondrocytes: The Terminal Stage of Differentiation in the Chondrogenic Cell Lineage?," Annals of the New York Academy of Sciences, vol. 599, no. 1, pp. 45-57, 1990.

[12] H. Madry, C. N. van Dijk, and M. Mueller-Gerbl, "The basic science of the subchondral bone," Knee Surgery, Sports Traumatology, Arthroscopy, vol. 18, no. 4, pp. 419-433, 2010 .

[13] G. Li et al., "Subchondral bone in osteoarthritis: insight into risk factors and microstructural changes," Arthritis Research \& Therapy, vol. 15, no. 6, p. 223, 2013.

[14] H. Imhof, M. Breitenseher, F. Kainberger, T. Rand, and S. Trattnig, "Importance of Subchondral Bone to Articular Cartilage in Health and Disease," Topics in Magnetic 
Resonance Imaging, vol. 10, no. 3, pp. 180-192, 1999.

[15] S. Suri and D. A. Walsh, "Osteochondral alterations in osteoarthritis," Bone, vol. 51, no. 2, pp. 204-211, 2012.

[16] S. Castañeda, J. A. Roman-Blas, R. Largo, and G. Herrero-Beaumont, "Subchondral bone as a key target for osteoarthritis treatment," Biochemical Pharmacology, vol. 83, no. 3, pp. 315-323, 2012.

[17] M. B. Goldring and S. R. Goldring, "Articular cartilage and subchondral bone in the pathogenesis of osteoarthritis," Annals of the New York Academy of Sciences, vol. 1192, no. 1, pp. 230-237, 2010.

[18] M. M. Hyttinen, J. Holopainen, P. René van Weeren, E. C. Firth, H. J. Helminen, and P. A. J. Brama, "Changes in collagen fibril network organization and proteoglycan distribution in equine articular cartilage during maturation and growth," Journal of Anatomy, vol. 215, no. 5, pp. 584-591, 2009.

[19] P. S. Eggli, W. Herrmann, E. B. Hunziker, and R. K. Schenk, "Matrix compartments in the growth plate of the proximal tibia of rats," The Anatomical Record, vol. 211, no. 3, pp. 246-257, 1985.

[20] F. Guilak and V. C. Mow, "The mechanical environment of the chondrocyte: a biphasic finite element model of cell-matrix interactions in articular cartilage," Journal of Biomechanics, vol. 33, no. 12, pp. 1663-1673, 2000.

[21] H. Muir, "The chondrocyte, architect of cartilage. Biomechanics, structure, function and molecular biology of cartilage matrix macromolecules," BioEssays, vol. 17, no. 12, pp. 1039-1048, 1995.

[22] C. R. Ethier and C. a. Simmons, "Introductory Biomechanics," in Introductory Biomechanics - from Cells to Organisms, Cambridge: Cambridge University Press, 2007, pp. 19-98.

[23] A. Maroudas, E. Wachtel, G. Grushko, E. P. Katz, and P. Weinberg, "The effect of osmotic and mechanical pressures on water partitioning in articular cartilage," Biochimica et Biophysica Acta (BBA) - General Subjects, vol. 1073, no. 2, pp. 285-294, 1991.

[24] P. A. Torzilli, "Influence of cartilage conformation on its equilibrium water partition," Journal of Orthopaedic Research, vol. 3, no. 4, pp. 473-483, 1985.

[25] V. C. Mow, S. C. Kuei, W. M. Lai, and C. G. Armstrong, "Biphasic Creep and Stress Relaxation of Articular Cartilage in Compression: Theory and Experiments," Journal of Biomechanical Engineering, vol. 102, no. 1, p. 73, 1980.

[26] K. Sato et al., "Type XXVI Collagen, a New Member of the Collagen Family, Is Specifically Expressed in the Testis and Ovary," Journal of Biological Chemistry, vol. 277, no. 40, pp. 37678-37684, 2002.

[27] Y.-J. Kim, L. J. Bonassar, and A. J. Grodzinsky, "The role of cartilage streaming potential, fluid flow and pressure in the stimulation of chondrocyte biosynthesis during dynamic compression," Journal of Biomechanics, vol. 28, no. 9, pp. 1055-1066, 1995. 
[28] P. J. Neame and F. P. Barry, "The link proteins,” Experientia, vol. 49, no. 5, pp. 393-402, 1993.

[29] W. M. Lai, J. S. Hou, and V. C. Mow, "A Triphasic Theory for the Swelling and Deformation Behaviors of Articular Cartilage," Journal of Biomechanical Engineering, vol. 113, no. 3, p. 245, 1991.

[30] G. A. Ateshian, W. H. Warden, J. J. Kim, R. P. Grelsamer, and V. C. Mow, "Finite deformation biphasic material properties of bovine articular cartilage from confined compression experiments," Journal of Biomechanics, vol. 30, no. 11-12, pp. 1157-1164, 1997.

[31] V. C. Mow, M. H. Holmes, and W. Michael Lai, "Fluid transport and mechanical properties of articular cartilage: A review," Journal of Biomechanics, vol. 17, no. 5, pp. 377-394, 1984.

[32] J. A. Buckwalter, “Articular Cartilage: Injuries and Potential for Healing," Journal of Orthopaedic \& Sports Physical Therapy, vol. 28, no. 4, pp. 192-202, 1998.

[33] E. H. Frank and A. J. Grodzinsky, "Cartilage electromechanics-I. Electrokinetic transduction and the effects of electrolyte $\mathrm{pH}$ and ionic strength," Journal of Biomechanics, vol. 20, no. 6, pp. 615-627, 1987.

[34] P. N. Tandon and R. Agarwal, "A study of nutritional transport in a synovial joint," Computers and Mathematics with Applications, vol. 17, no. 7, pp. 1131-1141, 1989.

[35] E. Han, S. S. Chen, S. M. Klisch, and R. L. Sah, "Contribution of Proteoglycan Osmotic Swelling Pressure to the Compressive Properties of Articular Cartilage," Biophysical Journal, vol. 101, no. 4, pp. 916-924, 2011.

[36] R. K. Korhonen, P. Julkunen, W. Wilson, and W. Herzog, "Importance of Collagen Orientation and Depth-Dependent Fixed Charge Densities of Cartilage on Mechanical Behavior of Chondrocytes," Journal of Biomechanical Engineering, vol. 130, no. 2, p. 021003, 2008.

[37] E. R. Myers, W. M. Lai, and V. C. Mow, "A Continuum Theory and an Experiment for the Ion-Induced Swelling Behavior of Articular Cartilage," Journal of Biomechanical Engineering, vol. 106, no. 2, p. 151, 1984.

[38] S. L.-Y. Woo, "Temperature Dependent Behavior of the Canine Medial Collateral Ligament," Journal of Biomechanical Engineering, vol. 109, no. 1, p. 68, 1987.

[39] S. Akizuki, V. C. Mow, F. Müller, J. C. Pita, D. S. Howell, and D. H. Manicourt, “Tensile properties of human knee joint cartilage: I. Influence of ionic conditions, weight bearing, and fibrillation on the tensile modulus," Journal of Orthopaedic Research, vol. 4, no. 4, pp. 379-392, 1986.

[40] V. Roth and V. C. Mow, "The intrinsic tensile behavior of the matrix of bovine articular cartilage and its variation with age.," The Journal of Bone \& Joint Surgery, vol. 62, no. 7, pp. 1102-1117, 1980.

[41] C. G. Armstrong and V. C. Mow, "Variations in the intrinsic mechanical properties of 
human articular cartilage with age, degeneration, and water content.," The Journal of Bone \& Joint Surgery, vol. 64, no. 1, pp. 88-94, 1982.

[42] J. W.-P. Michael, K. U. Schlüter-Brust, and P. Eysel, "The Epidemiology, Etiology, Diagnosis, and Treatment of Osteoarthritis of the Knee," Deutsches Aerzteblatt Online, vol. 107, no. 9, pp. 152-162, 2010.

[43] D. T. Felson, "Osteoarthritis: New Insights. Part 1: The Disease and Its Risk Factors," Annals of Internal Medicine, vol. 133, no. 8, p. 635, 2000.

[44] M. Blagojevic, C. Jinks, A. Jeffery, and K. P. Jordan, "Risk factors for onset of osteoarthritis of the knee in older adults: a systematic review and meta-analysis," Osteoarthritis and Cartilage, vol. 18, no. 1, pp. 24-33, 2010.

[45] M. van der Esch, M. Steultjens, H. Wieringa, H. Dinant, and J. Dekker, "Structural joint changes, malalignment, and laxity in osteoarthritis of the knee," Scandinavian Journal of Rheumatology, vol. 34, no. 4, pp. 298-301, 2005.

[46] H. J. Braun and G. E. Gold, "Diagnosis of osteoarthritis: Imaging," Bone, vol. 51, no. 2, pp. 278-288, 2012.

[47] M. D. Kohn, A. A. Sassoon, and N. D. Fernando, "Classifications in Brief: KellgrenLawrence Classification of Osteoarthritis," Clinical Orthopaedics and Related Research ${ }^{\circledR}$, vol. 474, no. 8, pp. 1886-1893, 2016.

[48] R. W. Wright et al., "Osteoarthritis Classification Scales: Interobserver Reliability and Arthroscopic Correlation," The Journal of Bone and Joint Surgery-American Volume, vol. 96, no. 14, pp. 1145-1151, 2014.

[49] I. Martin, B. Obradovic, L. E. Freed, and G. Vunjak-Novakovic, "Method for Quantitative Analysis of Glycosaminoglycan Distribution in Cultured Natural and Engineered Cartilage," Annals of Biomedical Engineering, vol. 27, no. 5, pp. 656-662, 1999.

[50] A. D. Speirs, P. E. Beaulé, A. Huang, and H. Frei, "Properties of the cartilage layer from the cam-type hip impingement deformity," Journal of Biomechanics, vol. 55, pp. 78-84, 2017.

[51] H. Muir, "Cartilage Structure and Metabolism and Basic Changes in Degenerative Joint Disease," Australian and New Zealand Journal of Medicine, vol. 8, pp. 1-5, 1978.

[52] M. K. Lotz, S. Otsuki, S. P. Grogan, R. Sah, R. Terkeltaub, and D. D’Lima, "Cartilage cell clusters," Arthritis \& Rheumatism, vol. 62, no. 8, pp. 2206-2218, 2010.

[53] M. A. Soltz, "Functional Tissue Engineering of Articular Cartilage Through Dynamic Loading of Chondrocyte-Seeded Agarose Gels," Journal of Biomechanical Engineering, vol. 122, no. 3, p. 252, 2000.

[54] R. K. June, R. Liu-Bryan, F. Long, and T. M. Griffin, "Emerging role of metabolic signaling in synovial joint remodeling and osteoarthritis," Journal of Orthopaedic Research, vol. 34, no. 12, pp. 2048-2058, 2016.

[55] G. Musumeci, "The Effect of Mechanical Loading on Articular Cartilage," Journal of 
Functional Morphology and Kinesiology, vol. 1, no. 2, pp. 154-161, 2016.

[56] J. Tehranzadeh, F. Booya, and J. Root, "Cartilage metabolism in osteoarthritis and the influence of viscosupplementation and steroid: a review," Acta Radiologica, vol. 46, no. 3, pp. 288-296, 2005.

[57] A. J. Grodzinsky, M. E. Levenston, M. Jin, and E. H. Frank, "Cartilage Tissue Remodeling in Response to Mechanical Forces," Annual Review of Biomedical Engineering, vol. 2, no. 1, pp. 691-713, 2000.

[58] F. Guilak, B. Meyer, A. Ratcliffe, and V. Mow, "The effects of matrix compression on proteoglycan metabolism in articular cartilage explants," Osteoarthritis and Cartilage, vol. 2, no. 2, pp. 91-101, 1994.

[59] Y. J. Kim, R. L. Y. Sah, A. J. Grodzinsky, A. H. K. Plaas, and J. D. Sandy, "Mechanical Regulation of Cartilage Biosynthetic Behavior: Physical Stimuli," Archives of Biochemistry and Biophysics, vol. 311, no. 1, pp. 1-12, 1994.

[60] P. A. Torzilli et al., "Characterization of cartilage metabolic response to static and dynamic stress using a mechanical explant test system," Journal of Biomechanics, vol. 30, no. 1, pp. 1-9, 1997.

[61] R. F. Loeser, "Integrins and chondrocyte-matrix interactions in articular cartilage," Matrix Biology, vol. 39, pp. 11-16, 2014.

[62] L. A. Fortier, J. U. Barker, E. J. Strauss, T. M. McCarrel, and B. J. Cole, "The Role of Growth Factors in Cartilage Repair," Clinical Orthopaedics and Related Research ${ }^{\circledR}$, vol. 469, no. 10, pp. 2706-2715, 2011.

[63] T. Heinola et al., "High mobility group box-1 (HMGB-1) in osteoarthritic cartilage.," Clinical and experimental rheumatology, vol. 28, no. 4, pp. 511-8, 2010.

[64] F. J. Blanco, I. Rego, and C. Ruiz-Romero, "The role of mitochondria in osteoarthritis," Nature Reviews Rheumatology, vol. 7, no. 3, pp. 161-169, 2011.

[65] M. Del Carlo and R. F. Loeser, "Cell death in osteoarthritis," Current Rheumatology Reports, vol. 10, no. 1, pp. 37-42, 2008.

[66] B. Caramés, N. Taniguchi, S. Otsuki, F. J. Blanco, and M. Lotz, “Autophagy is a protective mechanism in normal cartilage, and its aging-related loss is linked with cell death and osteoarthritis," Arthritis \& Rheumatism, vol. 62, no. 3, pp. 791-801, 2010.

[67] N. Taniguchi et al., "Aging-related loss of the chromatin protein HMGB2 in articular cartilage is linked to reduced cellularity and osteoarthritis," Proceedings of the National Academy of Sciences, vol. 106, no. 4, pp. 1181-1186, 2009.

[68] Q. Zheng, G. Zhou, R. Morello, Y. Chen, X. Garcia-Rojas, and B. Lee, "Type X collagen gene regulation by Runx2 contributes directly to its hypertrophic chondrocyte-specific expression in vivo," The Journal of Cell Biology, vol. 162, no. 5, pp. 833-842, 2003.

[69] P. Smeriglio, J. H. Lai, F. Yang, and N. Bhutani, "3D Hydrogel Scaffolds for Articular Chondrocyte Culture and Cartilage Generation," Journal of Visualized Experiments, no. 
104, 2015.

[70] E. H. Frank, M. Jin, A. M. Loening, M. E. Levenston, and A. J. Grodzinsky, “A versatile shear and compression apparatus for mechanical stimulation of tissue culture explants," Journal of Biomechanics, vol. 33, no. 11, pp. 1523-1527, 2000.

[71] H. Guo, S. A. Maher, and P. A. Torzilli, "A biphasic multiscale study of the mechanical microenvironment of chondrocytes within articular cartilage under unconfined compression," Journal of Biomechanics, vol. 47, no. 11, pp. 2721-2729, 2014.

[72] B. Kurz, M. Jin, P. Patwari, D. M. Cheng, M. W. Lark, and A. J. Grodzinsky, "Biosynthetic response and mechanical properties of articular cartilage after injurious compression," Journal of Orthopaedic Research, vol. 19, no. 6, pp. 1140-1146, 2001.

[73] R. Lane Smith et al., "Effects of shear stress on articular chondrocyte metabolism.," Biorheology, vol. 37, no. 1-2, pp. 95-107, 2000.

[74] M. S. Lee, M. C. D. Trindade, T. Ikenoue, D. J. Schurman, S. B. Goodman, and R. L. Smith, "Effects of shear stress on nitric oxide and matrix protein gene expression in human osteoarthritic chondrocytes in vitro.," Journal of orthopaedic research : official publication of the Orthopaedic Research Society, 2002.

[75] R. L. Smith, D. R. Carter, and D. J. Schurman, "Pressure and shear differentially alter human articular chondrocyte metabolism: A review," in Clinical Orthopaedics and Related Research, 2004.

[76] R. K. Korhonen and W. Herzog, "Depth-dependent analysis of the role of collagen fibrils, fixed charges and fluid in the pericellular matrix of articular cartilage on chondrocyte mechanics," Journal of Biomechanics, vol. 41, no. 2, pp. 480-485, 2008.

[77] S. C. Sibole and A. Erdemir, "Chondrocyte Deformations as a Function of Tibiofemoral Joint Loading Predicted by a Generalized High-Throughput Pipeline of Multi-Scale Simulations," PLOS ONE, vol. 7, no. 5, 2012.

[78] M. E. Levenston, E. H. Frank, and A. J. Grodzinsky, "Electrokinetic and Poroelastic Coupling During Finite Deformations of Charged Porous Media," Journal of Applied Mechanics, vol. 66, no. 2, p. 323, 1999.

[79] M. D. Buschmann, Y.-J. Kim, M. Wong, E. Frank, E. B. Hunziker, and A. J. Grodzinsky, "Stimulation of Aggrecan Synthesis in Cartilage Explants by Cyclic Loading Is Localized to Regions of High Interstitial Fluid Flow1," Archives of Biochemistry and Biophysics, vol. 366, no. 1, pp. 1-7, 1999.

[80] P. Wang, F. Zhu, Z. Tong, and K. Konstantopoulos, "Response of chondrocytes to shear stress: antagonistic effects of the binding partners Toll-like receptor 4 and caveolin-1.," FASEB journal : official publication of the Federation of American Societies for Experimental Biology, vol. 25, no. 10, pp. 3401-15, 2011.

[81] M. Sokabe, F. Sachs, and Z. Q. Jing, "Quantitative video microscopy of patch clamped membranes stress, strain, capacitance, and stretch channel activation," Biophysical Journal, vol. 59, no. 3, pp. 722-728, 1991. 
[82] Z. Abusara, R. Seerattan, A. Leumann, R. Thompson, and W. Herzog, "A novel method for determining articular cartilage chondrocyte mechanics in vivo," Journal of Biomechanics, vol. 44, no. 5, pp. 930-934, 2011.

[83] J. Mackerle, "Finite element modeling and simulations in orthopedics: a bibliography 1998-2005," Computer Methods in Biomechanics and Biomedical Engineering, vol. 9, no. 3, pp. 149-199, 2006.

[84] W. Wilson, C. C. van Donkelaar, R. van Rietbergen, and R. Huiskes, "The role of computational models in the search for the mechanical behavior and damage mechanisms of articular cartilage," Medical Engineering \& Physics, vol. 27, no. 10, pp. 810-826, 2005.

[85] A. Erdemir, S. McLean, W. Herzog, and A. J. van den Bogert, "Model-based estimation of muscle forces exerted during movements," Clinical Biomechanics, vol. 22, no. 2, pp. 131-154, 2007.

[86] T. Guillén, Q.-H. Zhang, G. Tozzi, A. Ohrndorf, H.-J. Christ, and J. Tong, "Compressive behaviour of bovine cancellous bone and bone analogous materials, microCT characterisation and FE analysis," Journal of the Mechanical Behavior of Biomedical Materials, vol. 4, no. 7, pp. 1452-1461, 2011.

[87] V. G. Kouznetsova, M. G. D. Geers, and W. A. M. Brekelmans, "Multi-scale second-order computational homogenization of multi-phase materials: a nested finite element solution strategy," Computer Methods in Applied Mechanics and Engineering, vol. 193, no. 48-51, pp. 5525-5550, 2004.

[88] G. A. Ateshian, S. Maas, and J. A. Weiss, "Multiphasic Finite Element Framework for Modeling Hydrated Mixtures With Multiple Neutral and Charged Solutes," Journal of Biomechanical Engineering, vol. 135, no. 11, p. 111001, 2013.

[89] S.-K. Han, S. Federico, A. Grillo, G. Giaquinta, and W. Herzog, "The Mechanical Behaviour of Chondrocytes Predicted with a Micro-structural Model of Articular Cartilage," Biomechanics and Modeling in Mechanobiology, vol. 6, no. 3, pp. 139-150, 2007.

[90] P. S. Donzelli, R. L. Spilker, G. A. Ateshian, and V. C. Mow, "Contact analysis of biphasic transversely isotropic cartilage layers and correlations with tissue failure," Journal of Biomechanics, vol. 32, no. 10, pp. 1037-1047, 1999.

[91] S. Federico, A. Grillo, G. La Rosa, G. Giaquinta, and W. Herzog, “A transversely isotropic, transversely homogeneous microstructural-statistical model of articular cartilage," Journal of Biomechanics, vol. 38, no. 10, pp. 2008-2018, 2005.

[92] P. Julkunen, W. Wilson, H. Isaksson, J. S. Jurvelin, W. Herzog, and R. K. Korhonen, “A Review of the Combination of Experimental Measurements and Fibril-Reinforced Modeling for Investigation of Articular Cartilage and Chondrocyte Response to Loading," Computational and Mathematical Methods in Medicine, vol. 2013, pp. 1-23, 2013.

[93] L. . Li, J. Soulhat, M. . Buschmann, and A. Shirazi-Adl, "Nonlinear analysis of cartilage in unconfined ramp compression using a fibril reinforced poroelastic model," Clinical 
Biomechanics, vol. 14, no. 9, pp. 673-682, 1999.

[94] W. Wilson, C. C. van Donkelaar, B. van Rietbergen, and R. Huiskes, "A fibril-reinforced poroviscoelastic swelling model for articular cartilage," Journal of Biomechanics, vol. 38, no. 6, pp. 1195-1204, 2005.

[95] F. Guilak, A. Ratcliffe, and V. C. Mow, "Chondrocyte deformation and local tissue strain in articular cartilage: A confocal microscopy study," Journal of Orthopaedic Research, vol. 13, no. 3, pp. 410-421, 1995.

[96] H. Guo and P. A. Torzilli, "Shape of chondrocytes within articular cartilage affects the solid but not the fluid microenvironment under unconfined compression," Acta Biomaterialia, vol. 29, pp. 170-179, 2016.

[97] V. Heinrich and R. E. Waugh, "A piconewton force transducer and its application to measurement of the bending stiffness of phospholipid membranes," Annals of Biomedical Engineering, vol. 24, no. 5, pp. 595-605, 1996.

[98] M. R. DiSilvestro and J. K. Suh, "A cross-validation of the biphasic poroviscoelastic model of articular cartilage in unconfined compression, indentation, and confined compression.," Journal of biomechanics, vol. 34, no. 4, pp. 519-25, 2001.

[99] M. Khoshgoftar, C. C. van Donkelaar, and K. Ito, "Mechanical stimulation to stimulate formation of a physiological collagen architecture in tissue-engineered cartilage: a numerical study.," Computer methods in biomechanics and biomedical engineering, vol. 14, no. 2, pp. 135-44, 2011.

[100] L. M. Kock, K. Ito, and C. C. van Donkelaar, "Sliding indentation enhances collagen content and depth-dependent matrix distribution in tissue-engineered cartilage constructs.," Tissue engineering Part A, vol. 19, no. 17-18, pp. 1949-59, 2013.

[101] H. Guo and R. L. Spilker, "Biphasic finite element modeling of hydrated soft tissue contact using an augmented Lagrangian method.," Journal of biomechanical engineering, vol. 133, no. 11, p. 111001, 2011.

[102] L. P. Li, J. T. M. Cheung, and W. Herzog, "Three-dimensional fibril-reinforced finite element model of articular cartilage," Medical \& Biological Engineering \& Computing, vol. 47, no. 6, pp. 607-615, Jun. 2009.

[103] G. Ateshian, L. Soslowsky, and V. Mow, "Quantitation of articular surface topography and cartilage thickness in knee joints using stereophotogrammetry," Journal of Biomechanics, vol. 24, no. 8, pp. 761-776, 1991.

[104] A. Connolly, D. FitzPatrick, J. Moulton, J. Lee, and A. Lerner, “Tibiofemoral cartilage thickness distribution and its correlation with anthropometric variables," Proceedings of the Institution of Mechanical Engineers, Part H: Journal of Engineering in Medicine, vol. 222, no. 1, pp. 29-39, 2008.

[105] M. A. Accardi, D. Dini, and P. M. Cann, "Experimental and numerical investigation of the behaviour of articular cartilage under shear loading - Interstitial fluid pressurisation and lubrication mechanisms," Tribology International, vol. 44, no. 5, pp. 565-578, 2011. 
[106] E. D. Bonnevie, V. J. Baro, L. Wang, and D. L. Burris, "In Situ Studies of Cartilage Microtribology: Roles of Speed and Contact Area," Tribology Letters, vol. 41, no. 1, pp. 83-95, 2011.

[107] G. A. Ateshian, S. Maas, and J. A. Weiss, "Finite Element Algorithm for Frictionless Contact of Porous Permeable Media Under Finite Deformation and Sliding," Journal of Biomechanical Engineering, vol. 132, no. 6, p. 061006, 2010.

[108] R. L. Spilker, J.-K. Suh, and V. C. Mow, “A Finite Element Analysis of the Indentation Stress-Relaxation Response of Linear Biphasic Articular Cartilage," Journal of Biomechanical Engineering, vol. 114, no. 2, p. 191, 1992.

[109] R. M. Schinagl, D. Gurskis, A. C. Chen, and R. L. Sah, "Depth-dependent confined compression modulus of full-thickness bovine articular cartilage," Journal of Orthopaedic Research, vol. 15, no. 4, pp. 499-506, 1997.

[110] S. Federico and W. Herzog, "On the anisotropy and inhomogeneity of permeability in articular cartilage," Biomechanics and Modeling in Mechanobiology, vol. 7, no. 5, pp. 367-378, 2008.

[111] S. A. Maas, B. J. Ellis, G. A. Ateshian, and J. A. Weiss, "FEBio: Finite Elements for Biomechanics," Journal of Biomechanical Engineering, vol. 134, no. 1, p. 011005, 2012.

[112] Q. Meng et al., "The effect of collagen fibril orientation on the biphasic mechanics of articular cartilage," Journal of the Mechanical Behavior of Biomedical Materials, vol. 65, pp. 439-453, 2017.

[113] M. D. Buschmann, J. Soulhat, A. Shirazi-Adl, J. S. Jurvelin, and E. B. Hunziker, "Confined compression of articular cartilage," Journal of Biomechanics, vol. 31, no. 2, pp. 171-178, 1997.

[114] M. H. Holmes and V. C. Mow, "The nonlinear characteristics of soft gels and hydrated connective tissues in ultrafiltration," Journal of Biomechanics, vol. 23, no. 11, pp. 11451156, 1990.

[115] F. Eckstein, M. Tieschky, S. Faber, K.-H. Englmeier, and M. Reiser, "Functional analysis of articular cartilage deformation, recovery, and fluid flow following dynamic exercise in vivo," Anatomy and Embryology, vol. 200, no. 4, pp. 419-424, 1999.

[116] S. Park, S. B. Nicoll, R. L. Mauck, and G. A. Ateshian, "Cartilage Mechanical Response under Dynamic Compression at Physiological Stress Levels Following Collagenase Digestion," Annals of Biomedical Engineering, vol. 36, no. 3, pp. 425-434, 2008.

[117] G. A. Ateshian, V. Rajan, N. O. Chahine, C. E. Canal, and C. T. Hung, "Modeling the Matrix of Articular Cartilage Using a Continuous Fiber Angular Distribution Predicts Many Observed Phenomena," Journal of Biomechanical Engineering, vol. 131, no. 6, p. 061003, 2009.

[118] K. E. Keenan et al., "New resource for the computation of cartilage biphasic material properties with the interpolant response surface method," Computer Methods in Biomechanics and Biomedical Engineering, vol. 12, no. 4, pp. 415-422, 2009. 
[119] E. M. Darling, S. Zauscher, and F. Guilak, "Viscoelastic properties of zonal articular chondrocytes measured by atomic force microscopy," Osteoarthritis and Cartilage, vol. 14, no. 6, pp. 571-579, 2006.

[120] F. Guilak, L. G. Alexopoulos, M. A. Haider, H. P. Ting-Beall, and L. A. Setton, "Zonal Uniformity in Mechanical Properties of the Chondrocyte Pericellular Matrix: Micropipette Aspiration of Canine Chondrons Isolated by Cartilage Homogenization," Annals of Biomedical Engineering, vol. 33, no. 10, pp. 1312-1318, 2005.

[121] P. Tanska, S. M. Turunen, S. K. Han, P. Julkunen, W. Herzog, and R. K. Korhonen, "Superficial Collagen Fibril Modulus and Pericellular Fixed Charge Density Modulate Chondrocyte Volumetric Behaviour in Early Osteoarthritis," Computational and Mathematical Methods in Medicine, pp. 1-14, 2013.

[122] K. E. Keenan, S. Pal, D. P. Lindsey, T. F. Besier, and G. S. Beaupre, "A viscoelastic constitutive model can accurately represent entire creep indentation tests of human patella cartilage," Journal of Applied Biomechanics, 2013.

[123] A. Erdemir, C. Bennetts, S. Davis, A. Reddy, and S. Sibole, "Multiscale cartilage biomechanics: Technical challenges in realizing a high-throughput modelling and simulation workflow," Interface Focus, 2015.

[124] A. D. Speirs, P. E. Beaulé, S. J. Ferguson, and H. Frei, "Stress distribution and consolidation in cartilage constituents is influenced by cyclic loading and osteoarthritic degeneration," Journal of Biomechanics, vol. 47, no. 10, pp. 2348-2353, 2014.

[125] M. E. Mononen et al., "Effect of superficial collagen patterns and fibrillation of femoral articular cartilage on knee joint mechanics-A 3D finite element analysis," Journal of Biomechanics, vol. 45, no. 3, pp. 579-587, 2012.

[126] L. G. Alexopoulos, G. M. Williams, M. L. Upton, L. A. Setton, and F. Guilak, "Osteoarthritic changes in the biphasic mechanical properties of the chondrocyte pericellular matrix in articular cartilage," Journal of Biomechanics, vol. 38, no. 3, pp. 509-517, 2005.

[127] O. R. Schätti, M. Marková, P. A. Torzilli, and L. M. Gallo, "Mechanical Loading of Cartilage Explants with Compression and Sliding Motion Modulates Gene Expression of Lubricin and Catabolic Enzymes," CARTILAGE, vol. 6, no. 3, pp. 185-193, 2015.

[128] H. Guo, J. C. Nickel, L. R. Iwasaki, and R. L. Spilker, "An Augmented Lagrangian Method for Sliding Contact of Soft Tissue," Journal of Biomechanical Engineering, vol. 134, no. 8, p. 084503, 2012.

[129] F. A. van de Loo, S. Kuiper, F. H. van Enckevort, O. J. Arntz, and W. B. van den Berg, "Interleukin-6 reduces cartilage destruction during experimental arthritis. A study in interleukin-6-deficient mice.," The American journal of pathology, vol. 151, no. 1, pp. 177-91, 1997.

[130] M. D. Buschmann, E. B. Hunziker, Y. J. Kim, and A. J. Grodzinsky, "Altered aggrecan synthesis correlates with cell and nucleus structure in statically compressed cartilage.," Journal of cell science, vol. 109, pp. 499-508, 1996. 
[131] M. Wong, M. Siegrist, and X. Cao, "Cyclic compression of articular cartilage explants is associated with progressive consolidation and altered expression pattern of extracellular matrix proteins," Matrix Biology, vol. 18, no. 4, pp. 391-399, 1999.

[132] P. M. Ragan et al., "Down-regulation of chondrocyte aggrecan and type-II Collagen gene expression correlates with increases in static compression magnitude and duration," Journal of Orthopaedic Research, vol. 17, no. 6, pp. 836-842, 1999.

[133] A. C. Chen and R. L. Sah, "Effect of static compression on proteoglycan biosynthesis by chondrocytes transplanted to articular cartilagein vitro," Journal of Orthopaedic Research, vol. 16 , no. 5, pp. 542-550, 1998.

[134] C. C.-B. Wang, C. T. Hung, and V. C. Mow, "An analysis of the effects of depthdependent aggregate modulus on articular cartilage stress-relaxation behavior in compression," Journal of Biomechanics, vol. 34, no. 1, pp. 75-84, 2001.

[135] L. P. Räsänen et al., "The effect of fixed charge density and cartilage swelling on mechanics of knee joint cartilage during simulated gait," Journal of Biomechanics, vol. 61, pp. 34-44, 2017.

[136] J. S. Hou, M. H. Holmes, W. M. Lai, and V. C. Mow, "Boundary Conditions at the Cartilage-Synovial Fluid Interface for Joint Lubrication and Theoretical Verifications," Journal of Biomechanical Engineering, vol. 111, no. 1, p. 78, 1989. 


\section{Appendices}

\section{Appendix A : Result Tables for Microscale Models of Healthy Cartilage}

Table A.1: Summary of selected result variables for the central superficial zone microscale model (A) of healthy tissue subjected to cyclic sliding indentation.

\begin{tabular}{|c|c|c|c|c|c|c|}
\hline \multirow[t]{2}{*}{ Region } & \multirow[t]{2}{*}{ Variable } & \multicolumn{2}{|c|}{ Average Maxima } & \multicolumn{2}{|c|}{ Average Minima } & \multirow{2}{*}{$\begin{array}{c}\begin{array}{c}\text { Amplitude } \\
\text { Change }\end{array} \\
\text { Cycle (8-1) }\end{array}$} \\
\hline & & Cycle 1 & Cycle 8 & Cycle 1 & Cycle 8 & \\
\hline \multirow{5}{*}{ ECM } & $1^{\text {st }}$ Principal Strain* & 0.0416 & 0.0474 & -0.0055 & -0.0072 & 0.0037 \\
\hline & $3^{\text {rd }}$ Principal Strain* & -0.0190 & -0.0174 & -0.0980 & -0.0930 & -0.0017 \\
\hline & Fluid Pressure (MPa) & 0.8426 & 0.8628 & -0.0465 & -0.0301 & 0.0019 \\
\hline & Total Fluid Flux (mm/s) & 0.0079 & 0.0066 & $2.7 \times 10^{-5}$ & $5.9 \times 10^{-5}$ & $-6.7 \times 10^{-4}$ \\
\hline & Volume Ratio* & 0.9964 & 0.9833 & 0.9381 & 0.9478 & -0.0114 \\
\hline \multirow{5}{*}{ PCM } & $1^{\text {st }}$ Principal Strain* & 0.0631 & 0.0706 & 0.0013 & -0.0016 & 0.0052 \\
\hline & $3^{\text {rd }}$ Principal Strain* & -0.0107 & -0.0108 & -0.1391 & -0.1333 & -0.0029 \\
\hline & Fluid Pressure (MPa) & 0.8132 & 0.8232 & -0.0382 & -0.0300 & 0.0009 \\
\hline & Total Fluid Flux (mm/s) & $3 \times 10^{-4}$ & $2 \times 10^{-4}$ & $6.2 \times 10^{-6}$ & $2.6 \times 10^{-6}$ & $-4.8 \times 10^{-5}$ \\
\hline & Volume Ratio* & 0.9987 & 0.9869 & 0.8906 & 0.9032 & -0.0122 \\
\hline \multirow{7}{*}{ Cell } & $1^{\text {st }}$ Principal Strain* & 0.0929 & 0.0935 & -0.0083 & -0.0109 & 0.0016 \\
\hline & $3^{\text {rd }}$ Principal Strain* & -0.0386 & -0.0542 & -0.1633 & -0.1642 & -0.0073 \\
\hline & Max. Shear Strain* & 0.1275 & 0.1287 & 0.0281 & 0.0216 & 0.0039 \\
\hline & Max. Shear Stress (MPa) & $4.1 \times 10^{-5}$ & $4.1 \times 10^{-5}$ & $9.3 \times 10^{-6}$ & $7.2 \times 10^{-6}$ & $1.1 \times 10^{-6}$ \\
\hline & Fluid Pressure (MPa) & 0.8091 & 0.8188 & -0.0387 & -0.0286 & -0.0003 \\
\hline & Total Fluid Flux (mm/s) & $1 \times 10^{-4}$ & $1 \times 10^{-4}$ & $7.9 \times 10^{-6}$ & $1.6 \times 10^{-5}$ & $-4.1 \times 10^{-6}$ \\
\hline & Volume Ratio* & 0.9854 & 0.9645 & 0.8977 & 0.9117 & -0.0174 \\
\hline
\end{tabular}


Table A.2: Summary of selected result variables for the central middle zone microscale model (B) of healthy tissue subjected to cyclic sliding indentation.

\begin{tabular}{|c|c|c|c|c|c|c|}
\hline \multirow[t]{2}{*}{ Region } & \multirow[t]{2}{*}{ Variable } & \multicolumn{2}{|c|}{ Average Maxima } & \multicolumn{2}{|c|}{ Average Minima } & \multirow{2}{*}{$\begin{array}{c}\text { Amplitude } \\
\text { Change }\end{array}$} \\
\hline & & Cycle 1 & Cycle 8 & Cycle 1 & Cycle 8 & \\
\hline \multirow{5}{*}{ ECM } & $1^{\text {st }}$ Principal Strain* & 0.0289 & 0.0282 & $3 \times 10^{-4}$ & -0.0035 & 0.0016 \\
\hline & $3^{\text {rd }}$ Principal Strain* & -0.0028 & -0.0075 & -0.0482 & -0.0578 & 0.0025 \\
\hline & Fluid Pressure (MPa) & 0.3168 & 0.3107 & -0.0024 & -0.0098 & $6 \times 10^{-4}$ \\
\hline & Total Fluid Flux $(\mathrm{mm} / \mathrm{s})$ & 0.0015 & 0.0014 & $5.1 \times 10^{-5}$ & $6.9 \times 10^{-5}$ & $-3.4 \times 10^{-5}$ \\
\hline & Volume Ratio* & 1.0030 & 0.9958 & 0.9926 & 0.9850 & $2 \times 10^{-4}$ \\
\hline \multirow{5}{*}{ РСМ } & $1^{\text {st }}$ Principal Strain* & 0.0415 & 0.0416 & $-3 \times 10^{-4}$ & -0.0038 & 0.0018 \\
\hline & $3^{\text {rd }}$ Principal Strain* & -0.0046 & -0.0075 & -0.0485 & -0.0572 & 0.0029 \\
\hline & Fluid Pressure (MPa) & 0.2976 & 0.2932 & -0.0013 & -0.0866 & 0.0404 \\
\hline & Total Fluid Flux $(\mathrm{mm} / \mathrm{s})$ & $9.6 \times 10^{-5}$ & $9.8 \times 10^{-5}$ & $2.1 \times 10^{-6}$ & $4.6 \times 10^{-6}$ & $1 \times 10^{-6}$ \\
\hline & Volume Ratio* & 1.015 & 0.9925 & 1.004 & 0.9793 & 0.0011 \\
\hline \multirow{7}{*}{ Cell } & $1^{\text {st }}$ Principal Strain* & 0.0631 & 0.0580 & 0.0028 & -0.0042 & $9.5 \times 10^{-4}$ \\
\hline & $3^{\text {rd }}$ Principal Strain* & 0.0019 & -0.0068 & -0.0784 & -0.0962 & 0.0045 \\
\hline & Max. Shear Strain* & 0.0698 & 0.0764 & 0.0030 & 0.0051 & 0.0022 \\
\hline & Max. Shear Stress (MPa) & $1.2 \times 10^{-5}$ & $1.3 \times 10^{-5}$ & $6.2 \times 10^{-7}$ & $8.9 \times 10^{-7}$ & $3.5 \times 10^{-7}$ \\
\hline & Fluid Pressure (MPa) & 0.2950 & 0.2910 & -0.0113 & -0.0866 & 0.0356 \\
\hline & Total Fluid Flux $(\mathrm{mm} / \mathrm{s})$ & $4.3 \times 10^{-5}$ & $2.5 \times 10^{-5}$ & $5.5 \times 10^{-6}$ & $3 \times 10^{-6}$ & $-7 \times 10^{-6}$ \\
\hline & Volume Ratio* & 1.0500 & 0.9957 & 1.0280 & 0.9751 & $-7 \times 10^{-4}$ \\
\hline
\end{tabular}


Table A.3: Summary of selected result variables for the central deep zone microscale model (C) of healthy tissue subjected to cyclic sliding indentation.

\begin{tabular}{|c|c|c|c|c|c|c|}
\hline \multirow[t]{2}{*}{ Region } & \multirow[t]{2}{*}{ Variable } & \multicolumn{2}{|c|}{ Average Maxima } & \multicolumn{2}{|c|}{ Average Minima } & \multirow{2}{*}{$\begin{array}{c}\text { Amplitude } \\
\text { Change }\end{array}$} \\
\hline & & Cycle 1 & Cycle 8 & Cycle 1 & Cycle 8 & \\
\hline \multirow{5}{*}{ ECM } & $1^{\text {st }}$ Principal Strain* & 0.0100 & 0.0113 & 0.0012 & $-9 \times 10^{-5}$ & 0.0013 \\
\hline & $3^{\text {rd }}$ Principal Strain* & -0.0018 & -0.0021 & -0.0179 & -0.0221 & 0.0019 \\
\hline & Fluid Pressure (MPa) & 0.1345 & 0.1371 & 0.0030 & -0.0033 & 0.0044 \\
\hline & Total Fluid Flux $(\mathrm{mm} / \mathrm{s})$ & $3.8 \times 10^{-4}$ & $4.3 \times 10^{-4}$ & $2.1 \times 10^{-5}$ & $1.7 \times 10^{-5}$ & $5.7 \times 10^{-4}$ \\
\hline & Volume Ratio* & 1.0010 & 0.9975 & 0.9994 & 0.9960 & $-5 \times 10^{-5}$ \\
\hline \multirow{5}{*}{ PCM } & $1^{\text {st }}$ Principal Strain* & 0.0173 & 0.0203 & 0.0012 & $4.4 \times 10^{-5}$ & 0.0020 \\
\hline & $3^{\text {rd }}$ Principal Strain* & -0.0035 & -0.0031 & -0.0179 & -0.0209 & 0.0017 \\
\hline & Fluid Pressure (MPa) & 0.1318 & 0.1342 & 0.0030 & -0.0038 & 0.0046 \\
\hline & Total Fluid Flux $(\mathrm{mm} / \mathrm{s})$ & $2.4 \times 10^{-5}$ & $3.1 \times 10^{-5}$ & $4.1 \times 10^{-7}$ & $4.2 \times 10^{-7}$ & $3.3 \times 10^{-6}$ \\
\hline & Volume Ratio* & 1.0090 & 1.0070 & 0.9963 & 0.9915 & 0.0014 \\
\hline \multirow{7}{*}{ Cell } & $1^{\text {st }}$ Principal Strain* & 0.0240 & 0.0257 & 0.0064 & 0.0036 & 0.0022 \\
\hline & $3^{\text {rd }}$ Principal Strain* & $-9 \times 10^{-4}$ & -0.0025 & -0.0285 & -0.0356 & 0.0027 \\
\hline & Max. Shear Strain* & 0.0260 & 0.0304 & 0.0038 & 0.0031 & 0.0025 \\
\hline & Max. Shear Stress (MPa) & $4.5 \times 10^{-6}$ & $5.3 \times 10^{-6}$ & $6.5 \times 10^{-7}$ & $5.4 \times 10^{-7}$ & $4.8 \times 10^{-7}$ \\
\hline & Fluid Pressure (MPa) & 0.1310 & 0.1334 & 0.0030 & -0.0037 & 0.0045 \\
\hline & Total Fluid Flux $(\mathrm{mm} / \mathrm{s})$ & $8.6 \times 10^{-6}$ & $7.7 \times 10^{-6}$ & $2 \times 10^{-6}$ & $2.4 \times 10^{-6}$ & $-6.5 \times 10^{-7}$ \\
\hline & Volume Ratio* & 1.0180 & 1.0130 & 1.0030 & 0.9954 & 0.0013 \\
\hline
\end{tabular}


Table A.4: Summary of selected result variables for the peripheral superficial zone microscale model (D) of healthy tissue subjected to cyclic sliding indentation.

\begin{tabular}{|c|c|c|c|c|c|c|}
\hline \multirow[t]{2}{*}{ Region } & \multirow[t]{2}{*}{ Variable } & \multicolumn{2}{|c|}{ Average Maxima } & \multicolumn{2}{|c|}{ Average Minima } & \multirow{2}{*}{$\begin{array}{c}\text { Amplitude } \\
\text { Change }\end{array}$} \\
\hline & & Cycle 1 & Cycle 8 & Cycle 1 & Cycle 8 & \\
\hline \multirow{5}{*}{ ECM } & $1^{\text {st }}$ Principal Strain* & 0.0467 & 0.0429 & $-7.5 \times 10^{-5}$ & -0.0053 & 0.0007 \\
\hline & $3^{\text {rd }}$ Principal Strain* & -0.0056 & -0.0150 & -0.0893 & -0.0953 & -0.0017 \\
\hline & Fluid Pressure (MPa) & 0.9614 & 0.8216 & -0.0334 & -0.0254 & -0.0739 \\
\hline & Total Fluid Flux $(\mathrm{mm} / \mathrm{s})$ & 0.0088 & 0.0078 & $1.0 \times 10^{-5}$ & $1.9 \times 10^{-4}$ & $-5.9 \times 10^{-4}$ \\
\hline & Volume Ratio* & 0.9938 & 0.9817 & 0.9489 & 0.9359 & $4.5 \times 10^{-4}$ \\
\hline \multirow{5}{*}{ PCM } & $1^{\text {st }}$ Principal Strain* & 0.0684 & 0.0641 & 0.0035 & $1.6 \times 10^{-4}$ & -0.0005 \\
\hline & $3^{\text {rd }}$ Principal Strain* & -0.0032 & -0.0121 & -0.1550 & -0.1542 & -0.0049 \\
\hline & Fluid Pressure (MPa) & 0.9245 & 0.7884 & -0.0308 & -0.0325 & -0.0672 \\
\hline & Total Fluid Flux $(\mathrm{mm} / \mathrm{s})$ & $2.7 \times 10^{-4}$ & $2.0 \times 10^{-4}$ & $1.8 \times 10^{-5}$ & $1.4 \times 10^{-5}$ & $-3.3 \times 10^{-5}$ \\
\hline & Volume Ratio* & 0.9980 & 0.9860 & 0.8945 & 0.8932 & -0.0054 \\
\hline \multirow{7}{*}{ Cell } & $1^{\text {st }}$ Principal Strain* & 0.1073 & 0.0906 & -0.0018 & -0.0081 & -0.0052 \\
\hline & $3^{\text {rd }}$ Principal Strain* & -0.0135 & -0.0488 & -0.1402 & -0.1667 & -0.0044 \\
\hline & Max. Shear Strain & 0.1218 & 0.1273 & 0.0037 & 0.0178 & -0.0043 \\
\hline & Max. Shear Stress (MPa) & $3.6 \times 10^{-5}$ & $4.0 \times 10^{-5}$ & $1.1 \times 10^{-6}$ & $5.9 \times 10^{-6}$ & $1.6 \times 10^{-5}$ \\
\hline & Fluid Pressure (MPa) & 0.9214 & 0.7845 & -0.0270 & -0.0305 & -0.0667 \\
\hline & Total Fluid Flux $(\mathrm{mm} / \mathrm{s})$ & $9.3 \times 10^{-5}$ & $1.1 \times 10^{-4}$ & $6 \times 10^{-6}$ & $1.7 \times 10^{-5}$ & $3.0 \times 10^{-6}$ \\
\hline & Volume Ratio* & 1.0155 & 0.9623 & 0.9526 & 0.9136 & -0.0071 \\
\hline
\end{tabular}


Table A.5: Summary of selected result variables for the peripheral middle zone microscale model (E) of healthy tissue subjected to cyclic sliding indentation.

\begin{tabular}{|c|c|c|c|c|c|c|}
\hline \multirow[t]{2}{*}{ Region } & \multirow[t]{2}{*}{ Variable } & \multicolumn{2}{|c|}{ Average Maxima } & \multicolumn{2}{|c|}{ Average Minima } & \multirow{2}{*}{$\begin{array}{c}\text { Amplitude } \\
\text { Change }\end{array}$} \\
\hline & & Cycle 1 & Cycle 8 & Cycle 1 & Cycle 8 & \\
\hline \multirow{5}{*}{ ECM } & $1^{\text {st }}$ Principal Strain* & 0.0300 & 0.0289 & $8.2 \times 10^{-4}$ & -0.0042 & 0.0019 \\
\hline & $3^{\text {rd }}$ Principal Strain* & -0.0021 & -0.0081 & -0.0487 & -0.0594 & 0.0023 \\
\hline & Fluid Pressure (MPa) & 0.3343 & 0.2945 & -0.0042 & -0.0126 & -0.0157 \\
\hline & Total Fluid Flux $(\mathrm{mm} / \mathrm{s})$ & 0.0018 & 0.0016 & $4.7 \times 10^{-5}$ & $7.8 \times 10^{-5}$ & $-1.1 \times 10^{-4}$ \\
\hline & Volume Ratio* & 1.0060 & 0.9918 & 0.9952 & 0.9828 & $-9.0 \times 10^{-4}$ \\
\hline \multirow{5}{*}{ PCM } & $1^{\text {st }}$ Principal Strain* & 0.0423 & 0.0417 & -0.0017 & -0.0048 & 0.0012 \\
\hline & $3^{\text {rd }}$ Principal Strain* & -0.0034 & -0.0076 & -0.0505 & -0.0591 & 0.0022 \\
\hline & Fluid Pressure (MPa) & 0.3129 & 0.2765 & -0.0028 & -0.0114 & -0.0139 \\
\hline & Total Fluid Flux $(\mathrm{mm} / \mathrm{s})$ & $1.2 \times 10^{-4}$ & $1.1 \times 10^{-4}$ & $2.5 \times 10^{-6}$ & $4.0 \times 10^{-6}$ & $-6.0 \times 10^{-6}$ \\
\hline & Volume Ratio* & 1.0190 & 1.0040 & 0.9914 & 0.9754 & 0.0095 \\
\hline \multirow{7}{*}{ Cell } & $1^{\text {st }}$ Principal Strain* & 0.0677 & 0.0615 & $4.0 \times 10^{-4}$ & -0.0068 & $5.0 \times 10^{-4}$ \\
\hline & $3^{\text {rd }}$ Principal Strain* & $-7.0 \times 10^{-4}$ & -0.0133 & -0.0764 & -0.0953 & 0.0031 \\
\hline & Max. Shear Strain* & 0.0718 & 0.0776 & 0.0023 & 0.0051 & 0.0020 \\
\hline & Max. Shear Stress (MPa) & $1.2 \times 10^{-5}$ & $1.3 \times 10^{-5}$ & $4.0 \times 10^{-7}$ & $9.1 \times 10^{-7}$ & $2.4 \times 10^{-7}$ \\
\hline & Fluid Pressure (MPa) & 0.3098 & 0.2734 & -0.0028 & -0.0114 & -0.0112 \\
\hline & Total Fluid Flux $(\mathrm{mm} / \mathrm{s})$ & $5.7 \times 10^{-5}$ & $3.9 \times 10^{-5}$ & $1.4 \times 10^{-6}$ & $2.3 \times 10^{-6}$ & $-8.6 \times 10^{-6}$ \\
\hline & Volume Ratio* & 1.0560 & 1.0270 & 0.9932 & 0.9702 & -0.0030 \\
\hline
\end{tabular}


Table A.6: Summary of selected result variables for the peripheral deep zone microscale model (F) of healthy tissue subjected to cyclic sliding indentation.

\begin{tabular}{|c|c|c|c|c|c|c|}
\hline \multirow[t]{2}{*}{ Region } & \multirow[t]{2}{*}{ Variable } & \multicolumn{2}{|c|}{${ }^{1}$ Average Maxima } & \multicolumn{2}{|c|}{${ }^{1}$ Average Minima } & \multirow{2}{*}{$\begin{array}{c}\text { Amplitude } \\
\text { Change }\end{array}$} \\
\hline & & Cycle 1 & Cycle 8 & Cycle 1 & Cycle 8 & \\
\hline \multirow{5}{*}{ ECM } & $1^{\text {st }}$ Principal Strain* & 0.0109 & 0.0112 & $2.4 \times 10^{-4}$ & $-7.4 \times 10^{-7}$ & $2.7 \times 10^{-4}$ \\
\hline & $3^{\text {rd }}$ Principal Strain* & $-6.0 \times 10^{-4}$ & -0.0018 & -0.0184 & -0.0214 & $9.0 \times 10^{-4}$ \\
\hline & Fluid Pressure (MPa) & 0.1382 & 0.1238 & $7.6 \times 10^{-5}$ & -0.0050 & -0.0046 \\
\hline & Total Fluid Flux $(\mathrm{mm} / \mathrm{s})$ & $4.0 \times 10^{-4}$ & $4.2 \times 10^{-4}$ & $9.9 \times 10^{-6}$ & $6.5 \times 10^{-6}$ & $1.2 \times 10^{-5}$ \\
\hline & Volume Ratio* & 1.0020 & 0.9981 & 0.9988 & 0.9955 & $-3.0 \times 10^{-4}$ \\
\hline \multirow{5}{*}{ РСМ } & $1^{\text {st }}$ Principal Strain* & 0.0188 & 0.0193 & $3.0 \times 10^{-4}$ & -0.0010 & $9.0 \times 10^{-4}$ \\
\hline & $3^{\text {rd }}$ Principal Strain* & $-9.5 \times 10^{-4}$ & -0.0027 & -0.0188 & -0.0212 & $3.2 \times 10^{-4}$ \\
\hline & Fluid Pressure (MPa) & 0.1355 & 0.1260 & $-5.0 \times 10^{-5}$ & -0.0050 & -0.0022 \\
\hline & Total Fluid Flux $(\mathrm{mm} / \mathrm{s})$ & $2.7 \times 10^{-5}$ & $3.1 \times 10^{-5}$ & $1.7 \times 10^{-7}$ & $2.4 \times 10^{-7}$ & $1.9 \times 10^{-6}$ \\
\hline & Volume Ratio* & 1.0100 & 1.0070 & 0.9954 & 0.9906 & $9.0 \times 10^{-4}$ \\
\hline \multirow{7}{*}{ Cell } & $1^{\text {st }}$ Principal Strain* & 0.0283 & 0.0272 & 0.0013 & $-8.0 \times 10^{-4}$ & $5.0 \times 10^{-4}$ \\
\hline & $3^{\text {rd }}$ Principal Strain* & $-6.2 \times 10^{-4}$ & -0.0029 & -0.0273 & -0.0326 & 0.0015 \\
\hline & Max. Shear Strain* & 0.0277 & 0.0298 & 0.0013 & 0.0011 & 0.0012 \\
\hline & Max. Shear Stress (MPa) & $4.7 \times 10^{-6}$ & $5.2 \times 10^{-6}$ & $2.3 \times 10^{-7}$ & $1.9 \times 10^{-7}$ & $2.7 \times 10^{-7}$ \\
\hline & Fluid Pressure (MPa) & 0.1348 & 0.1252 & $-5.0 \times 10^{-5}$ & -0.0050 & -0.0023 \\
\hline & Total Fluid Flux $(\mathrm{mm} / \mathrm{s})$ & $7.1 \times 10^{-6}$ & $5.9 \times 10^{-6}$ & $1.8 \times 10^{-6}$ & $1.3 \times 10^{-6}$ & $-3.5 \times 10^{-7}$ \\
\hline & Volume Ratio* & 1.0240 & 1.0170 & 0.9987 & 0.9927 & $-5.0 \times 10^{-4}$ \\
\hline
\end{tabular}


Table A.7: Summary of selected result variables for the central superficial zone microscale model (A) of healthy tissue subjected to axial quasi-static indentation.

\begin{tabular}{|c|c|c|c|c|}
\hline Region & Variable & Peak 1 & $\begin{array}{c}\text { Peak 2 } \\
(t=100 s)\end{array}$ & Peak (2-1) \\
\hline \multirow{5}{*}{$\mathbf{E C M}$} & $1^{\text {st }}$ Principal Strain* & 0.0349 & 0.0025 & -0.0324 \\
\hline & $3^{\text {rd }}$ Principal Strain* & -0.1044 & -0.2269 & -0.1225 \\
\hline & Fluid Pressure (MPa) & 0.7294 & 0.0606 & -0.6688 \\
\hline & Total Fluid Flux (mm/s) & 0.0036 & $3 \times 10^{-5}$ & -0.0036 \\
\hline & Volume Ratio* & 1.0100 & 0.7349 & -0.2751 \\
\hline \multirow{5}{*}{ PCM } & $1^{\text {st }}$ Principal Strain* & 0.0535 & 0.0313 & -0.0222 \\
\hline & $3^{\text {rd }}$ Principal Strain* & -0.1292 & -0.2457 & -0.1165 \\
\hline & Fluid Pressure (MPa) & 0.7136 & 0.0609 & -0.6527 \\
\hline & Total Fluid Flux (mm/s) & $3 \times 10^{-4}$ & $3.8 \times 10^{-6}$ & $-3 \times 10^{-4}$ \\
\hline & Volume Ratio* & 1.0100 & 0.7502 & -0.2598 \\
\hline \multirow{7}{*}{ Cell } & $1^{\text {st }}$ Principal Strain* & 0.1097 & -0.0050 & -0.1147 \\
\hline & $3^{\text {rd }}$ Principal Strain* & -0.1664 & -0.4208 & -0.2544 \\
\hline & Max. Shear Strain & 0.1369 & 0.2079 & 0.0710 \\
\hline & Max. Shear Stress (MPa) & $4.2 \times 10^{-5}$ & $1.6 \times 10^{-4}$ & $1.2 \times 10^{-4}$ \\
\hline & Fluid Pressure (MPa) & 0.7130 & 0.0609 & -0.6521 \\
\hline & Total Fluid Flux (mm/s) & $1.9 \times 10^{-4}$ & $2.8 \times 10^{-6}$ & $-1.9 \times 10^{-4}$ \\
\hline & Volume Ratio* & 1.0150 & 0.3893 & 0.6257 \\
\hline \multicolumn{5}{|c|}{$\begin{array}{l}\text { *Variable is dimensionless. } \\
\text { Right far column shows how these values change from the loading to } \\
\text { relaxation phase. For } 3^{\text {rd }} \text { principal strain only, positive and negative changes } \\
\text { indicate a decrease and increase in compression respectively. }\end{array}$} \\
\hline
\end{tabular}


Table A.8: Summary of selected result variables for the central middle zone microscale model (B) of healthy tissue subjected to axial quasi-static indentation.

\begin{tabular}{|c|c|c|c|c|}
\hline Region & Variable & Peak 1 & $\begin{array}{c}\text { Peak 2 } \\
(t=100 s)\end{array}$ & Peak (2-1) \\
\hline \multirow{5}{*}{ ECM } & $1^{\text {st }}$ Principal Strain* & 0.0283 & 0.0173 & -0.0110 \\
\hline & $3^{\text {rd }}$ Principal Strain* & -0.0461 & -0.1159 & -0.0698 \\
\hline & Fluid Pressure (MPa) & 0.3049 & 0.0643 & -0.2406 \\
\hline & Total Fluid Flux (mm/s) & 0.0015 & $2.1 \times 10^{-5}$ & -0.0015 \\
\hline & Volume Ratio* & 1.0040 & 0.9051 & -0.0989 \\
\hline \multirow{5}{*}{ PCM } & $1^{\text {st }}$ Principal Strain* & 0.0407 & 0.0269 & -0.0138 \\
\hline & $3^{\text {rd }}$ Principal Strain* & -0.0464 & -0.1134 & -0.0670 \\
\hline & Fluid Pressure (MPa) & 0.2861 & 0.0646 & -0.2215 \\
\hline & Total Fluid Flux (mm/s) & $9 \times 10^{-5}$ & $2 \times 10^{-6}$ & $-8.8 \times 10^{-5}$ \\
\hline & Volume Ratio* & 1.0160 & 0.9129 & -0.1031 \\
\hline \multirow{7}{*}{ Cell } & $1^{\text {st }}$ Principal Strain* & 0.0744 & 0.0483 & -0.0261 \\
\hline & $3^{\text {rd }}$ Principal Strain* & -0.0753 & -0.1945 & -0.1192 \\
\hline & Max. Shear Strain* & 0.0763 & 0.1214 & 0.0451 \\
\hline & Max. Shear Stress (MPa) & $1.3 \times 10^{-5}$ & $2.5 \times 10^{-5}$ & $7 \times 10^{-6}$ \\
\hline & Fluid Pressure (MPa) & 0.2835 & 0.0646 & -0.2189 \\
\hline & Total Fluid Flux (mm/s) & $9 \times 10^{-5}$ & $2 \times 10^{-6}$ & $-8.8 \times 10^{-5}$ \\
\hline & Volume Ratio* & 1.0560 & 0.8558 & -0.2002 \\
\hline \multicolumn{5}{|c|}{$\begin{array}{l}\text { *Variable is dimensionless. } \\
\text { Right far column shows how these values change from the loading to } \\
\text { relaxation phase. For } 3^{\text {rd }} \text { principal strain only, positive and negative changes } \\
\text { indicate a decrease and increase in compression respectively. }\end{array}$} \\
\hline
\end{tabular}


Table A.9: Summary of selected result variables for the central deep zone microscale model (C) of healthy tissue subjected to axial quasi-static indentation.

\begin{tabular}{|c|c|c|c|c|}
\hline Region & Variable & Peak 1 & $\begin{array}{c}\text { Peak 2 } \\
(t=100 s)\end{array}$ & Peak (2-1) \\
\hline \multirow{5}{*}{ ECM } & $1^{\text {st }}$ Principal Strain* & 0.0092 & 0.0062 & -0.0030 \\
\hline & $3^{\text {rd }}$ Principal Strain* & -0.0175 & -0.0293 & -0.0118 \\
\hline & Fluid Pressure (MPa) & 0.1298 & 0.0617 & -0.0681 \\
\hline & Total Fluid Flux (mm/s) & $1.9 \times 10^{-4}$ & $1 \times 10^{-5}$ & $-1.8 \times 10^{-4}$ \\
\hline & Volume Ratio* & 1.0010 & 0.9821 & -0.0189 \\
\hline \multirow{5}{*}{ PCM } & $1^{\text {st }}$ Principal Strain* & 0.0164 & 0.0113 & -0.0051 \\
\hline & $3^{\text {rd }}$ Principal Strain* & -0.0163 & -0.0256 & -0.0093 \\
\hline & Fluid Pressure (MPa) & 0.1275 & 0.0615 & -0.0660 \\
\hline & Total Fluid Flux (mm/s) & $1.8 \times 10^{-5}$ & $1 \times 10^{-7}$ & $-1.8 \times 10^{-5}$ \\
\hline & Volume Ratio* & 1.0080 & 0.9882 & -0.0198 \\
\hline \multirow{7}{*}{ Cell } & $1^{\text {st }}$ Principal Strain* & 0.0310 & 0.0221 & -0.0089 \\
\hline & $3^{\text {rd }}$ Principal Strain* & -0.0287 & -0.0477 & -0.0190 \\
\hline & Max. Shear Strain* & 0.0286 & 0.0348 & 0.0062 \\
\hline & Max. Shear Stress (MPa) & $4.8 \times 10^{-6}$ & $6.2 \times 10^{-6}$ & $1.4 \times 10^{-6}$ \\
\hline & Fluid Pressure (MPa) & 0.1268 & 0.0615 & -0.0653 \\
\hline & Total Fluid Flux (mm/s) & $1.1 \times 10^{-5}$ & $1.4 \times 10^{-6}$ & $-9.6 \times 10^{-6}$ \\
\hline & Volume Ratio* & 1.0220 & 0.9857 & -0.0363 \\
\hline \multicolumn{5}{|c|}{$\begin{array}{l}\text { *Variable is dimensionless. } \\
\text { Right far column shows how these values change from the loading to } \\
\text { relaxation phase. For } 3^{\text {rd }} \text { principal strain only, positive and negative changes } \\
\text { indicate a decrease and increase in compression respectively. }\end{array}$} \\
\hline
\end{tabular}


Table A.10: Summary of selected result variables for the peripheral superficial zone microscale model (D) of healthy tissue subjected to axial quasi-static indentation.

\begin{tabular}{|c|c|c|c|c|}
\hline Region & Variable & Peak 1 & $\begin{array}{c}\text { Peak 2 } \\
(t=100 s)\end{array}$ & Peak (2-1) \\
\hline \multirow{5}{*}{ ECM } & $1^{\text {st }}$ Principal Strain* & 0.0112 & 0.0524 & 0.0412 \\
\hline & $3^{\text {rd }}$ Principal Strain* & -0.0296 & -0.0404 & -0.0108 \\
\hline & Fluid Pressure (MPa) & $1.1 \times 10^{-4}$ & 0.0017 & 0.0016 \\
\hline & Total Fluid Flux (mm/s) & $3.6 \times 10^{-4}$ & $5.9 \times 10^{-5}$ & $-3.0 \times 10^{-4}$ \\
\hline & Volume Ratio* & 0.9781 & 1.0150 & 0.0369 \\
\hline \multirow{5}{*}{ PCM } & $1^{\text {st }}$ Principal Strain* & 0.0082 & 0.0509 & 0.0427 \\
\hline & $3^{\text {rd }}$ Principal Strain* & -0.0253 & -0.0439 & -0.0186 \\
\hline & Fluid Pressure (MPa) & 0.0018 & 0.0025 & $7.0 \times 10^{-4}$ \\
\hline & Total Fluid Flux (mm/s) & $1.8 \times 10^{-5}$ & $3.3 \times 10^{-6}$ & $-1.5 \times 10^{-5}$ \\
\hline & Volume Ratio* & 0.9796 & 1.0100 & 0.0304 \\
\hline \multirow{7}{*}{ Cell } & $1^{\text {st }}$ Principal Strain* & 0.0113 & 0.0668 & 0.0555 \\
\hline & $3^{\text {rd }}$ Principal Strain* & -0.0235 & 0.0077 & 0.0312 \\
\hline & Max. Shear Strain & 0.0029 & 0.0089 & 0.0060 \\
\hline & Max. Shear Stress (MPa) & $8.9 \times 10^{-7}$ & $2.7 \times 10^{-6}$ & $1.8 \times 10^{-6}$ \\
\hline & Fluid Pressure (MPa) & 0.0018 & 0.0026 & $8.0 \times 10^{-4}$ \\
\hline & Total Fluid Flux (mm/s) & $4.2 \times 10^{-6}$ & $5.5 \times 10^{-6}$ & $1.3 \times 10^{-6}$ \\
\hline & Volume Ratio* & 0.9929 & 1.0950 & 0.1021 \\
\hline \multicolumn{5}{|c|}{$\begin{array}{l}\text { *Variable is dimensionless. } \\
\text { Right far column shows how these values change from the loading to } \\
\text { relaxation phase. For } 3^{\text {rd }} \text { principal strain only, positive and negative changes } \\
\text { indicate a decrease and increase in compression respectively. }\end{array}$} \\
\hline
\end{tabular}


Table A.11: Summary of selected result variables for the peripheral middle zone microscale model (E) of healthy tissue subjected to axial quasi-static indentation.

\begin{tabular}{|c|c|c|c|c|}
\hline Region & Variable & Peak 1 & $\begin{array}{c}\text { Peak 2 } \\
(t=100 s)\end{array}$ & Peak (2-1) \\
\hline \multirow{5}{*}{ ECM } & $1^{\text {st }}$ Principal Strain* & 0.0021 & 0.0018 & -0.0192 \\
\hline & $3^{\text {rd }}$ Principal Strain* & -0.0034 & -0.0021 & 0.0013 \\
\hline & Fluid Pressure (MPa) & 0.0074 & 0.0128 & 0.0053 \\
\hline & Total Fluid Flux (mm/s) & $4.6 \times 10^{-5}$ & $6.6 \times 10^{-5}$ & $2.0 \times 10^{-5}$ \\
\hline & Volume Ratio* & 1.0000 & 1.0010 & 0.0000 \\
\hline \multirow{5}{*}{ PCM } & $1^{\text {st }}$ Principal Strain* & 0.0043 & 0.0025 & -0.0018 \\
\hline & $3^{\text {rd }}$ Principal Strain* & -0.0055 & -0.0033 & 0.0022 \\
\hline & Fluid Pressure (MPa) & 0.0078 & 0.0134 & 0.0056 \\
\hline & Total Fluid Flux (mm/s) & $9.0 \times 10^{-7}$ & $2.3 \times 10^{-6}$ & $1.4 \times 10^{-6}$ \\
\hline & Volume Ratio* & 0.9986 & 0.9994 & $8.0 \times 10^{-4}$ \\
\hline \multirow{7}{*}{ Cell } & $1^{\text {st }}$ Principal Strain* & 0.0064 & 0.0052 & -0.0012 \\
\hline & $3^{\text {rd }}$ Principal Strain* & -0.0063 & -0.0038 & 0.0025 \\
\hline & Max. Shear Strain* & 0.0064 & 0.0045 & -0.0019 \\
\hline & Max. Shear Stress (MPa) & $1.1 \times 10^{-6}$ & $7.8 \mathrm{e}-7$ & $-3.2 \times 10^{-7}$ \\
\hline & Fluid Pressure (MPa) & 0.0078 & 0.0135 & 0.0057 \\
\hline & Total Fluid Flux (mm/s) & $1.1 \times 10^{-6}$ & $4.9 \times 10^{-6}$ & $3.8 \times 10^{-6}$ \\
\hline & Volume Ratio* & 1.0040 & 1.0050 & 0.0010 \\
\hline \multicolumn{5}{|c|}{$\begin{array}{l}\text { *Variable is dimensionless. } \\
\text { Right far column shows how these values change from the loading to } \\
\text { relaxation phase. For } 3^{\text {rd }} \text { principal strain only, positive and negative changes } \\
\text { indicate a decrease and increase in compression respectively. }\end{array}$} \\
\hline
\end{tabular}


Table A.12: Summary of selected result variables for the peripheral deep zone microscale model (F) of healthy tissue subjected to axial quasi-static indentation.

\begin{tabular}{|c|c|c|c|c|}
\hline Region & Variable & Peak 1 & $\begin{array}{c}\text { Peak 2 } \\
(t=100 s)\end{array}$ & Peak (2-1) \\
\hline \multirow{5}{*}{ ECM } & $1^{\text {st }}$ Principal Strain* & 0.0030 & 0.0031 & $1.0 \times 10^{-4}$ \\
\hline & $3^{\text {rd }}$ Principal Strain* & -0.0045 & -0.0050 & $-5.0 \times 10^{-4}$ \\
\hline & Fluid Pressure (MPa) & 0.0138 & 0.0193 & 0.0055 \\
\hline & Total Fluid Flux (mm/s) & $7.5 \times 10^{-5}$ & $6.5 \times 10^{-5}$ & $-1.0 \times 10^{-5}$ \\
\hline & Volume Ratio* & 0.9997 & 0.9993 & $-4.0 \times 10^{-4}$ \\
\hline \multirow{5}{*}{ PCM } & $1^{\text {st }}$ Principal Strain* & 0.0033 & 0.0036 & $3.0 \times 10^{-4}$ \\
\hline & $3^{\text {rd }}$ Principal Strain* & -0.0077 & -0.0088 & -0.0011 \\
\hline & Fluid Pressure (MPa) & 0.0137 & 0.0193 & 0.0056 \\
\hline & Total Fluid Flux (mm/s) & $1.3 \times 10^{-6}$ & $1.2 \times 10^{-6}$ & $-1.0 \times 10^{-7}$ \\
\hline & Volume Ratio* & 0.9978 & 0.9973 & $-5.0 \times 10^{-4}$ \\
\hline \multirow{7}{*}{ Cell } & $1^{\text {st }}$ Principal Strain* & 0.0091 & 0.0086 & $-5.0 \times 10^{-4}$ \\
\hline & $3^{\text {rd }}$ Principal Strain* & -0.0077 & -0.0083 & $-6.0 \times 10^{-4}$ \\
\hline & Max. Shear Strain* & 0.0085 & 0.0085 & 0.0000 \\
\hline & Max. Shear Stress (MPa) & $1.5 \times 10^{-6}$ & $1.5 \times 10^{-6}$ & 0.0000 \\
\hline & Fluid Pressure (MPa) & 0.0137 & 0.0193 & 0.0056 \\
\hline & Total Fluid Flux (mm/s) & $5.3 \times 10^{-6}$ & $4.9 \times 10^{-6}$ & $-4.0 \times 10^{-7}$ \\
\hline & Volume Ratio* & 1.0050 & 1.0040 & -0.0010 \\
\hline \multicolumn{5}{|c|}{$\begin{array}{l}\text { *Variable is dimensionless. } \\
\text { Right far column shows how these values change from the loading to } \\
\text { relaxation phase. For } 3^{\text {rd }} \text { principal strain only, positive and negative changes } \\
\text { indicate a decrease and increase in compression respectively. }\end{array}$} \\
\hline
\end{tabular}




\section{Appendix B : Result Tables for Microscale Models of OA Cartilage}

Table B.1: Summary of selected result variables for the central superficial zone microscale model (A) of OA tissue subjected to cyclic sliding indentation.

\begin{tabular}{|c|c|c|c|c|c|c|}
\hline \multirow[t]{2}{*}{ Region } & \multirow[t]{2}{*}{ Variable } & \multicolumn{2}{|c|}{${ }^{1}$ Average Maxima } & \multicolumn{2}{|c|}{${ }^{1}$ Average Minima } & \multirow{2}{*}{$\begin{array}{c}\text { Amplitude } \\
\text { Change }\end{array}$} \\
\hline & & Cycle 1 & Cycle 8 & Cycle 1 & Cycle 8 & \\
\hline \multirow{5}{*}{ ECM } & $1^{\text {st }}$ Principal Strain* & 0.0496 & 0.0550 & -0.0047 & -0.0074 & 0.0045 \\
\hline & $3^{\text {rd }}$ Principal Strain* & -0.0187 & -0.0179 & -0.1037 & -0.1015 & -0.0007 \\
\hline & Fluid Pressure (MPa) & 0.6497 & 0.6488 & -0.0343 & -0.0251 & -0.0051 \\
\hline & Total Fluid Flux (mm/s) & 0.0060 & 0.0059 & $4.6 \times 10^{-5}$ & $4.1 \times 10^{-5}$ & $-4.8 \times 10^{-5}$ \\
\hline & Volume Ratio* & 0.9978 & 0.9835 & 0.9485 & 0.9496 & -0.0077 \\
\hline \multirow{5}{*}{ PCM } & $1^{\text {st }}$ Principal Strain* & 0.0762 & 0.0845 & 0.0018 & -0.0018 & 0.0059 \\
\hline & $3^{\text {rd }}$ Principal Strain* & -0.0101 & -0.0096 & -0.1635 & -0.1581 & -0.0024 \\
\hline & Fluid Pressure (MPa) & 0.6272 & 0.6230 & -0.0301 & -0.0248 & -0.0047 \\
\hline & Total Fluid Flux (mm/s) & $3.4 \times 10^{-4}$ & $3.1 \times 10^{-4}$ & $6.2 \times 10^{-6}$ & $3.2 \times 10^{-6}$ & $-1.4 \times 10^{-5}$ \\
\hline & Volume Ratio* & 0.9975 & 0.9893 & 0.8954 & 0.8974 & -0.0051 \\
\hline \multirow{7}{*}{ Cell } & $1^{\text {st }}$ Principal Strain* & 0.1180 & 0.1095 & -0.0074 & -0.0105 & -0.0027 \\
\hline & $3^{\text {rd }}$ Principal Strain* & -0.0541 & -0.0528 & -0.1821 & -0.1793 & $-7.5 \times 10^{-4}$ \\
\hline & Max. Shear Strain* & 0.1471 & 0.1438 & 0.0268 & 0.0214 & 0.0011 \\
\hline & Max. Shear Stress (MPa) & $4.6 \times 10^{-5}$ & $4.6 \times 10^{-5}$ & $8.8 \times 10^{-6}$ & $7.1 \times 10^{-6}$ & $8.5 \times 10^{-7}$ \\
\hline & Fluid Pressure (MPa) & 0.6246 & 0.6199 & -0.0298 & -0.0243 & -0.0051 \\
\hline & Total Fluid Flux (mm/s) & $2.4 \times 10^{-4}$ & $1.9 \times 10^{-4}$ & $1.9 \times 10^{-5}$ & $2.7 \times 10^{-5}$ & $-2.9 \times 10^{-5}$ \\
\hline & Volume Ratio* & 0.9910 & 0.9641 & 0.9061 & 0.9117 & -0.0163 \\
\hline
\end{tabular}


Table B.2: Summary of selected result variables for the central middle zone microscale model (B) of OA tissue subjected to cyclic sliding indentation.

\begin{tabular}{|c|c|c|c|c|c|c|}
\hline \multirow[t]{2}{*}{ Region } & \multirow[t]{2}{*}{ Variable } & \multicolumn{2}{|c|}{${ }^{1}$ Average Maxima } & \multicolumn{2}{|c|}{${ }^{1}$ Average Minima } & \multirow{2}{*}{$\begin{array}{c}\text { Amplitude } \\
\text { Change }\end{array}$} \\
\hline & & Cycle 1 & Cycle 8 & Cycle 1 & Cycle 8 & \\
\hline \multirow{5}{*}{ ECM } & $1^{\text {st }}$ Principal Strain* & 0.0401 & 0.0397 & $5.4 \times 10^{-4}$ & -0.0040 & 0.0021 \\
\hline & $3^{\text {rd }}$ Principal Strain* & -0.0038 & -0.0073 & -0.0664 & -0.0758 & 0.0029 \\
\hline & Fluid Pressure (MPa) & 0.2730 & 0.2645 & -0.0014 & -0.0082 & $-8.5 \times 10^{-4}$ \\
\hline & Total Fluid Flux $(\mathrm{mm} / \mathrm{s})$ & 0.0015 & 0.0014 & $5.2 \times 10^{-5}$ & $6.9 \times 10^{-5}$ & $-5.8 \times 10^{-5}$ \\
\hline & Volume Ratio* & 1.0040 & 0.9933 & 0.9954 & 0.9843 & $2 \times 10^{-4}$ \\
\hline \multirow{5}{*}{ PCM } & $1^{\text {st }}$ Principal Strain* & 0.0573 & 0.0575 & $4.6 \times 10^{-4}$ & -0.0050 & 0.0028 \\
\hline & $3^{\text {rd }}$ Principal Strain* & -0.0067 & -0.0080 & -0.0711 & -0.0792 & 0.0034 \\
\hline & Fluid Pressure (MPa) & 0.2576 & 0.2505 & $-7.6 e-4$ & -0.0073 & $-2.8 \times 10^{-4}$ \\
\hline & Total Fluid Flux (mm/s) & $1.8 \times 10^{-4}$ & $1.7 \times 10^{-4}$ & $6.3 \times 10^{-6}$ & $6.0 \times 10^{-6}$ & $-4.8 \times 10^{-6}$ \\
\hline & Volume Ratio* & 1.0130 & 1.0000 & 0.9911 & 0.9789 & $-4.0 \times 10^{-4}$ \\
\hline \multirow{7}{*}{ Cell } & $1^{\text {st }}$ Principal Strain* & 0.0905 & 0.0828 & 0.0042 & -0.0053 & $9.0 \times 10^{-4}$ \\
\hline & $3^{\text {rd }}$ Principal Strain* & -0.0051 & -0.0106 & -0.1090 & -0.1229 & 0.0042 \\
\hline & Max. Shear Strain* & 0.0985 & 0.1021 & 0.0058 & 0.0041 & 0.0026 \\
\hline & Max. Shear Stress (MPa) & $1.6 \times 10^{-5}$ & $1.7 \times 10^{-5}$ & $1 \times 10^{-6}$ & $7.4 \times 10^{-7}$ & $6.3 \times 10^{-7}$ \\
\hline & Fluid Pressure (MPa) & 0.2557 & 0.2493 & $-8 \times 10^{-7}$ & -0.0073 & $4.5 \times 10^{-4}$ \\
\hline & Total Fluid Flux (mm/s) & $8.8 \times 10^{-5}$ & $6.5 \times 10^{-5}$ & $9.7 \times 10^{-6}$ & $7.8 \times 10^{-6}$ & $-1.1 \times 10^{-5}$ \\
\hline & Volume Ratio* & 1.0540 & 1.0370 & 0.9949 & 0.9728 & 0.0025 \\
\hline
\end{tabular}


Table B.3: Summary of selected result variables for the central deep zone microscale model (C) of OA tissue subjected to cyclic sliding indentation.

\begin{tabular}{|c|c|c|c|c|c|c|}
\hline \multirow[t]{2}{*}{ Region } & \multirow[t]{2}{*}{ Variable } & \multicolumn{2}{|c|}{${ }^{1}$ Average Maxima } & \multicolumn{2}{|c|}{${ }^{1}$ Average Minima } & \multirow{2}{*}{$\begin{array}{c}\begin{array}{c}\text { Amplitude } \\
\text { Change }\end{array} \\
\text { Cycle (8-1) }\end{array}$} \\
\hline & & Cycle 1 & Cycle 8 & Cycle 1 & Cycle 8 & \\
\hline \multirow{5}{*}{ ECM } & $1^{\text {st }}$ Principal Strain* & 0.0141 & 0.0158 & 0.0020 & $3.7 \times 10^{-4}$ & 0.0016 \\
\hline & $3^{\text {rd }}$ Principal Strain* & -0.0029 & -0.0032 & -0.0258 & -0.0304 & 0.0022 \\
\hline & Fluid Pressure (MPa) & 0.1231 & 0.1219 & 0.0030 & -0.0020 & 0.0019 \\
\hline & Total Fluid Flux (mm/s) & $3.6 \times 10^{-4}$ & $3.8 \times 10^{-4}$ & $2.4 \times 10^{-5}$ & $2.3 \times 10^{-5}$ & $1.1 \times 10^{-5}$ \\
\hline & Volume Ratio* & 1.0010 & 0.9972 & 0.9993 & 0.9955 & 0.0000 \\
\hline \multirow{5}{*}{ PCM } & $1^{\text {st }}$ Principal Strain* & 0.0245 & 0.0275 & 0.0018 & $7.4 \times 10^{-4}$ & 0.0020 \\
\hline & $3^{\text {rd }}$ Principal Strain* & -0.0054 & -0.0048 & -0.0262 & -0.0293 & 0.0018 \\
\hline & Fluid Pressure (MPa) & 0.1211 & 0.1198 & 0.0030 & -0.0033 & 0.0025 \\
\hline & Total Fluid Flux (mm/s) & $3.8 \times 10^{-5}$ & $4.1 \times 10^{-5}$ & $8.6 \times 10^{-7}$ & $1.8 \times 10^{-6}$ & $1.0 \times 10^{-6}$ \\
\hline & Volume Ratio* & 1.0090 & 1.0060 & 0.9943 & 0.9894 & $9.5 \times 10^{-4}$ \\
\hline \multirow{7}{*}{ Cell } & $1^{\text {st }}$ Principal Strain* & 0.0347 & 0.0355 & 0.0093 & 0.0054 & 0.0023 \\
\hline & $3^{\text {rd }}$ Principal Strain* & -0.0033 & -0.0055 & -0.0412 & -0.0490 & 0.0028 \\
\hline & Max. Shear Strain* & 0.0378 & 0.0418 & 0.0064 & 0.0056 & 0.0024 \\
\hline & Max. Shear Stress (MPa) & $6.5 \times 10^{-6}$ & $7.1 \times 10^{-6}$ & $1.1 \times 10^{-6}$ & $9.8 \times 10^{-7}$ & $3.6 \times 10^{-7}$ \\
\hline & Fluid Pressure (MPa) & 0.1206 & 0.1194 & 0.0030 & -0.0033 & 0.0025 \\
\hline & Total Fluid Flux (mm/s) & $1.9 \times 10^{-5}$ & $1.8 \times 10^{-5}$ & $3.1 \times 10^{-6}$ & $5.2 \times 10^{-6}$ & $-1.6 \times 10^{-6}$ \\
\hline & Volume Ratio* & 1.0240 & 1.0180 & 1.0040 & 0.9945 & 0.0018 \\
\hline
\end{tabular}


Table B.4: Summary of selected result variables for the peripheral superficial zone microscale model (D) of OA tissue subjected to cyclic sliding indentation.

\begin{tabular}{|c|c|c|c|c|c|c|}
\hline \multirow[t]{2}{*}{ Region } & \multirow[t]{2}{*}{ Variable } & \multicolumn{2}{|c|}{${ }^{1}$ Average Maxima } & \multicolumn{2}{|c|}{${ }^{1}$ Average Minima } & \multirow{2}{*}{$\begin{array}{c}\begin{array}{c}\text { Amplitude } \\
\text { Change }\end{array} \\
\text { Cycle (8-1) }\end{array}$} \\
\hline & & Cycle 1 & Cycle 8 & Cycle 1 & Cycle 8 & \\
\hline \multirow{5}{*}{ ECM } & $1^{\text {st }}$ Principal Strain* & 0.0553 & 0.0512 & -0.0031 & -0.0071 & $-5.0 \times 10^{-5}$ \\
\hline & $3^{\text {rd }}$ Principal Strain* & -0.0103 & -0.0148 & -0.1033 & -0.1069 & $-4.5 \times 10^{-4}$ \\
\hline & Fluid Pressure (MPa) & 0.7150 & 0.6223 & -0.0284 & -0.0309 & -0.0167 \\
\hline & Total Fluid Flux (mm/s) & 0.0083 & 0.0062 & $3.6 \times 10^{-5}$ & $2.7 \times 10^{-5}$ & -0.0010 \\
\hline & Volume Ratio* & 1.0010 & 0.9826 & 0.9519 & 0.9397 & -0.0031 \\
\hline \multirow{5}{*}{ РCM } & $1^{\text {st }}$ Principal Strain* & 0.0793 & 0.0765 & 0.0011 & -0.0017 & 0.0000 \\
\hline & $3^{\text {rd }}$ Principal Strain* & -0.0057 & -0.0086 & -0.1749 & -0.1650 & -0.0064 \\
\hline & Fluid Pressure (MPa) & 0.6895 & 0.5984 & -0.0264 & -0.0302 & -0.0436 \\
\hline & Total Fluid Flux $(\mathrm{mm} / \mathrm{s})$ & $3.9 \times 10^{-4}$ & $3.0 \times 10^{-4}$ & $4.6 \times 10^{-6}$ & $4.4 \times 10^{-6}$ & $-4.5 \times 10^{-5}$ \\
\hline & Volume Ratio* & 0.9983 & 0.9904 & 0.8871 & 0.8915 & -0.0062 \\
\hline \multirow{7}{*}{ Cell } & $1^{\text {st }}$ Principal Strain* & 0.1227 & 0.1113 & -0.0054 & -0.0089 & -0.0040 \\
\hline & $3^{\text {rd }}$ Principal Strain* & -0.0178 & -0.0468 & -0.1782 & -0.1953 & -0.0060 \\
\hline & Max. Shear Strain* & 0.1491 & 0.1535 & 0.0155 & 0.0195 & $2.0 \times 10^{-4}$ \\
\hline & Max. Shear Stress (MPa) & $4.6 \times 10^{-5}$ & $4.9 \times 10^{-5}$ & $4.9 \times 10^{-6}$ & $6.4 \times 10^{-6}$ & $3.0 \times 10^{-7}$ \\
\hline & Fluid Pressure (MPa) & 0.6870 & 0.5957 & -0.0258 & -0.0299 & -0.0436 \\
\hline & Total Fluid Flux (mm/s) & $2.0 \times 10^{-4}$ & $2.0 \times 10^{-4}$ & $1.9 \times 10^{-5}$ & $3.1 \times 10^{-5}$ & $-6 . \times 10^{-6}$ \\
\hline & Volume Ratio* & 1.0130 & 0.9647 & 0.9171 & 0.8869 & -0.0091 \\
\hline
\end{tabular}


Table B.5: Summary of selected result variables for the peripheral middle zone microscale model (E) of OA tissue subjected to cyclic sliding indentation.

\begin{tabular}{|c|c|c|c|c|c|c|}
\hline \multirow[t]{2}{*}{ Region } & \multirow[t]{2}{*}{ Variable } & \multicolumn{2}{|c|}{${ }^{1}$ Average Maxima } & \multicolumn{2}{|c|}{${ }^{1}$ Average Minima } & \multirow{2}{*}{$\begin{array}{c}\text { Amplitude } \\
\text { Change }\end{array}$} \\
\hline & & Cycle 1 & Cycle 8 & Cycle 1 & Cycle 8 & \\
\hline \multirow{5}{*}{ ECM } & $1^{\text {st }}$ Principal Strain* & 0.0422 & 0.0391 & $-5.0 \times 10^{-4}$ & -0.0042 & $3.0 \times 10^{-4}$ \\
\hline & $3^{\text {rd }}$ Principal Strain* & -0.0019 & -0.0080 & -0.0662 & -0.0765 & 0.0021 \\
\hline & Fluid Pressure (MPa) & 0.2843 & 0.2503 & -0.0020 & -0.0104 & -0.0128 \\
\hline & Total Fluid Flux $(\mathrm{mm} / \mathrm{s})$ & 0.0016 & 0.0014 & $3.9 \times 10^{-5}$ & $6.6 \times 10^{-5}$ & $-1.1 \times 10^{-4}$ \\
\hline & Volume Ratio* & 1.0020 & 0.9857 & 0.9969 & 0.9830 & -0.0012 \\
\hline \multirow{5}{*}{ PCM } & $1^{\text {st }}$ Principal Strain* & 0.0590 & 0.0546 & -0.0017 & -0.0050 & $-5.5 \times 10^{-4}$ \\
\hline & $3^{\text {rd }}$ Principal Strain* & -0.0033 & -0.0084 & -0.0690 & -0.0782 & 0.0021 \\
\hline & Fluid Pressure (MPa) & 0.2683 & 0.2374 & -0.0018 & -0.0090 & -0.0118 \\
\hline & Total Fluid Flux $(\mathrm{mm} / \mathrm{s})$ & $1.8 \times 10^{-4}$ & $1.5 \times 10^{-4}$ & $4.8 \times 10^{-6}$ & $4.8 \times 10^{-6}$ & $-1.5 \times 10^{-4}$ \\
\hline & Volume Ratio* & 1.0080 & 0.9910 & 0.9945 & 0.9788 & $-6.5 \times 10^{-4}$ \\
\hline \multirow{7}{*}{ Cell } & $1^{\text {st }}$ Principal Strain* & 0.0981 & 0.0897 & $6.0 \times 10^{-4}$ & -0.0073 & $-2.5 \times 10^{-4}$ \\
\hline & $3^{\text {rd }}$ Principal Strain* & -0.0034 & -0.0156 & -0.1076 & -0.1223 & 0.00125 \\
\hline & Max. Shear Strain* & 0.1028 & 0.1059 & 0.0021 & 0.0049 & $1.5 \times 10^{-4}$ \\
\hline & Max. Shear Stress (MPa) & $1.7 \times 10^{-5}$ & $1.8 \times 10^{-5}$ & $3.8 \times 10^{-7}$ & $9.4 \times 10^{-7}$ & $2.2 \times 10^{-7}$ \\
\hline & Fluid Pressure (MPa) & 0.2663 & 0.2357 & -0.0018 & -0.0090 & -0.0117 \\
\hline & Total Fluid Flux $(\mathrm{mm} / \mathrm{s})$ & $1.3 \times 10^{-4}$ & $9.0 \times 10^{-5}$ & $2.5 \times 10^{-6}$ & $4.9 \times 10^{-6}$ & $-2.1 \times 10^{-5}$ \\
\hline & Volume Ratio* & 1.0480 & 1.0180 & 0.9936 & 0.9687 & -0.0025 \\
\hline
\end{tabular}


Table B.6: Summary of selected result variables for the peripheral deep zone microscale model (F) of OA tissue subjected to cyclic sliding indentation.

\begin{tabular}{|c|c|c|c|c|c|c|}
\hline \multirow[t]{2}{*}{ Region } & \multirow[t]{2}{*}{ Variable } & \multicolumn{2}{|c|}{${ }^{1}$ Average Maxima } & \multicolumn{2}{|c|}{${ }^{1}$ Average Minima } & \multirow{2}{*}{$\begin{array}{c}\text { Amplitude } \\
\text { Change }\end{array}$} \\
\hline & & Cycle 1 & Cycle 8 & Cycle 1 & Cycle 8 & \\
\hline \multirow{5}{*}{ ECM } & $1^{\text {st }}$ Principal Strain* & 0.0159 & 0.0160 & $3.2 \times 10^{-4}$ & $-7.0 \times 10^{-4}$ & $5.6 \times 10^{-4}$ \\
\hline & $3^{\text {rd }}$ Principal Strain* & $-7.6 \times 10^{-4}$ & -0.0021 & -0.0264 & -0.0295 & $8.8 \times 10^{-4}$ \\
\hline & Fluid Pressure (MPa) & 0.1262 & 0.1161 & $-1.5 \times 10^{-4}$ & -0.0046 & -0.0028 \\
\hline & Total Fluid Flux (mm/s) & $3.8 \times 10^{-4}$ & $3.8 \times 10^{-4}$ & $1.1 \times 10^{-5}$ & $1.0 \times 10^{-5}$ & $5.0 \times 10^{-7}$ \\
\hline & Volume Ratio* & 1.0030 & 0.9981 & 0.9986 & 0.9945 & $-4.0 \times 10^{-4}$ \\
\hline \multirow{5}{*}{ PCM } & $1^{\text {st }}$ Principal Strain* & 0.0266 & 0.0268 & $4.4 \times 10^{-4}$ & $-2.7 \times 10^{-4}$ & $4.5 \times 10^{-4}$ \\
\hline & $3^{\text {rd }}$ Principal Strain* & -0.0011 & -0.0032 & -0.0271 & -0.0295 & $1.5 \times 10^{-4}$ \\
\hline & Fluid Pressure (MPa) & 0.1242 & 0.1142 & $-1.5 \times 10^{-4}$ & -0.0046 & -0.0028 \\
\hline & Total Fluid Flux $(\mathrm{mm} / \mathrm{s})$ & $4.0 \times 10^{-5}$ & $4.2 \times 10^{-5}$ & $1.0 \times 10^{-6}$ & $1.0 \times 10^{-6}$ & $1.0 \times 10^{-6}$ \\
\hline & Volume Ratio* & 1.0120 & 1.0070 & 0.9933 & 0.9885 & $-1.0 \times 10^{-4}$ \\
\hline \multirow{7}{*}{ Cell } & $1^{\text {st }}$ Principal Strain* & 0.0403 & 0.0384 & 0.0019 & $-7.6 \times 10^{-4}$ & $3.8 \times 10^{-4}$ \\
\hline & $3^{\text {rd }}$ Principal Strain* & -0.0022 & -0.0044 & -0.0399 & -0.0457 & 0.0018 \\
\hline & Max. Shear Strain* & 0.0401 & 0.0420 & 0.0021 & 0.0018 & 0.0011 \\
\hline & Max. Shear Stress (MPa) & $6.8 \times 10^{-6}$ & $7.2 \times 10^{-6}$ & $3.7 \mathrm{e}-7$ & $3.1 \times 10^{-7}$ & $2.3 \times 10^{-7}$ \\
\hline & Fluid Pressure (MPa) & 0.1237 & 0.1137 & $-1.5 e-4$ & -0.0046 & -0.0028 \\
\hline & Total Fluid Flux $(\mathrm{mm} / \mathrm{s})$ & $1.5 \times 10^{-5}$ & $1.3 \times 10^{-5}$ & $4 e-6$ & $3 \times 10^{-6}$ & $-5.0 \times 10^{-7}$ \\
\hline & Volume Ratio* & 1.0330 & 1.0230 & 0.9986 & 0.9911 & -0.0013 \\
\hline
\end{tabular}


Table B.7: Summary of selected result variables for the central superficial zone microscale model (A) of OA tissue subjected to axial quasi-static indentation.

\begin{tabular}{|c|c|c|c|c|}
\hline Region & Variable & Peak 1 & $\begin{array}{c}\text { Peak 2 } \\
(t=100 s)\end{array}$ & Peak (2-1) \\
\hline \multirow{5}{*}{ ECM } & $1^{\text {st }}$ Principal Strain* & 0.0440 & 0.0021 & -0.0419 \\
\hline & $3^{\text {rd }}$ Principal Strain* & -0.1069 & -0.2303 & -0.1234 \\
\hline & Fluid Pressure (MPa) & 0.5974 & 0.0574 & -0.5400 \\
\hline & Total Fluid Flux (mm/s) & 0.0032 & $3.2 \times 10^{-5}$ & -0.0032 \\
\hline & Volume Ratio* & 1.0130 & 0.7298 & 0.2832 \\
\hline \multirow{5}{*}{ PCM } & $1^{\text {st }}$ Principal Strain* & 0.0681 & 0.0639 & -0.0042 \\
\hline & $3^{\text {rd }}$ Principal Strain* & -0.1431 & -0.2767 & -0.1336 \\
\hline & Fluid Pressure (MPa) & 0.5835 & 0.0576 & -0.5259 \\
\hline & Total Fluid Flux (mm/s) & $4.0 \times 10^{-4}$ & $5.1 \times 10^{-6}$ & $-4 \times 10^{-4}$ \\
\hline & Volume Ratio* & 1.0080 & 0.7090 & -0.2990 \\
\hline \multirow{7}{*}{ Cell } & $1^{\text {st }}$ Principal Strain* & 0.1342 & 0.0118 & -0.1224 \\
\hline & $3^{\text {rd }}$ Principal Strain* & -0.2190 & -0.4320 & -0.2130 \\
\hline & Max. Shear Strain & 0.1770 & 0.2219 & 0.0449 \\
\hline & Max. Shear Stress (MPa) & $5.7 \times 10^{-5}$ & $1.8 \mathrm{e}-4$ & $1.2 \times 10^{-4}$ \\
\hline & Fluid Pressure (MPa) & 0.5819 & 0.0577 & -0.5242 \\
\hline & Total Fluid Flux (mm/s) & $3.1 \times 10^{-4}$ & $5.1 \times 10^{-6}$ & $-3.0 \times 10^{-4}$ \\
\hline & Volume Ratio* & 1.0150 & 0.3734 & -0.6416 \\
\hline \multicolumn{5}{|c|}{$\begin{array}{l}\text { *Variable is dimensionless. } \\
\text { Right far column shows how these values change from the loading to } \\
\text { relaxation phase. For } 3^{\text {rd }} \text { principal strain only, positive and negative changes } \\
\text { indicate a decrease and increase in compression respectively. }\end{array}$} \\
\hline
\end{tabular}


Table B.8: Summary of selected result variables for the central middle zone microscale model (B) of OA tissue subjected to axial quasi-static indentation.

\begin{tabular}{|c|c|c|c|c|}
\hline Region & Variable & Peak 1 & $\begin{array}{c}\text { Peak } 2 \\
(t=100 s)\end{array}$ & Peak (2-1) \\
\hline \multirow{5}{*}{ ECM } & $1^{\text {st }}$ Principal Strain* & 0.0410 & 0.0232 & -0.0178 \\
\hline & $3^{\text {rd }}$ Principal Strain* & -0.0643 & -0.1317 & -0.0674 \\
\hline & Fluid Pressure (MPa) & 0.2682 & 0.0608 & -0.2074 \\
\hline & Total Fluid Flux (mm/s) & 0.0015 & $2.8 \times 10^{-5}$ & -0.0015 \\
\hline & Volume Ratio* & 1.0060 & 0.8962 & -0.1098 \\
\hline \multirow{5}{*}{ РCM } & $1^{\text {st }}$ Principal Strain* & 0.0571 & 0.0358 & -0.0213 \\
\hline & $3^{\text {rd }}$ Principal Strain* & -0.0677 & -0.1390 & -0.0713 \\
\hline & Fluid Pressure (MPa) & 0.2528 & 0.0611 & -0.1917 \\
\hline & Total Fluid Flux (mm/s) & $1.9 \times 10^{-4}$ & $3.5 \times 10^{-6}$ & $-1.9 \times 10^{-4}$ \\
\hline & Volume Ratio* & 1.0150 & 0.8936 & -0.1214 \\
\hline \multirow{7}{*}{ Cell } & $1^{\text {st }}$ Principal Strain* & 0.1070 & 0.0663 & -0.0407 \\
\hline & $3^{\text {rd }}$ Principal Strain* & -0.1425 & -0.2213 & -0.0788 \\
\hline & Max. Shear Strain* & 0.1231 & 0.1438 & 0.0207 \\
\hline & Max. Shear Stress (MPa) & $2.1 \times 10^{-5}$ & $2.9 \times 10^{-5}$ & $8.0 \times 10^{-6}$ \\
\hline & Fluid Pressure (MPa) & 0.2507 & 0.0612 & -0.1895 \\
\hline & Total Fluid Flux (mm/s) & $1.9 \times 10^{-4}$ & $2.7 \times 10^{-6}$ & $-1.8 \times 10^{-4}$ \\
\hline & Volume Ratio* & 1.0520 & 0.8442 & -0.2078 \\
\hline \multicolumn{5}{|c|}{$\begin{array}{l}\text { *Variable is dimensionless. } \\
\text { Right far column shows how these values change from the loading to } \\
\text { relaxation phase. For } 3^{\text {rd }} \text { principal strain only, positive and negative changes } \\
\text { indicate a decrease and increase in compression respectively. }\end{array}$} \\
\hline
\end{tabular}


Table B.9: Summary of selected result variables for the central deep zone microscale model (C) of OA tissue subjected to axial quasi-static indentation.

\begin{tabular}{|c|c|c|c|c|}
\hline Region & Variable & Peak 1 & $\begin{array}{c}\text { Peak 2 } \\
(t=100 s)\end{array}$ & Peak (2-1) \\
\hline \multirow{5}{*}{ ECM } & $1^{\text {st }}$ Principal Strain* & 0.0138 & 0.0090 & -0.0048 \\
\hline & $3^{\text {rd }}$ Principal Strain* & -0.0255 & -0.0376 & -0.0121 \\
\hline & Fluid Pressure (MPa) & 0.1213 & 0.0591 & -0.0622 \\
\hline & Total Fluid Flux (mm/s) & $1.7 \times 10^{-4}$ & $9.6 \times 10^{-6}$ & $-1.6 \times 10^{-4}$ \\
\hline & Volume Ratio* & 1.0010 & 0.9787 & -0.0223 \\
\hline \multirow{5}{*}{ РCM } & $1^{\text {st }}$ Principal Strain* & 0.0238 & 0.0161 & -0.0077 \\
\hline & $3^{\text {rd }}$ Principal Strain* & -0.0241 & -0.0351 & -0.0110 \\
\hline & Fluid Pressure (MPa) & 0.1195 & 0.0591 & -0.0604 \\
\hline & Total Fluid Flux (mm/s) & $3.2 \times 10^{-5}$ & $7.3 \times 10^{-7}$ & $-3.1 \times 10^{-5}$ \\
\hline & Volume Ratio* & 1.0090 & 0.9843 & -0.0247 \\
\hline \multirow{7}{*}{ Cell } & $1^{\text {st }}$ Principal Strain* & 0.0457 & 0.0316 & -0.0141 \\
\hline & $3^{\text {rd }}$ Principal Strain* & -0.0419 & -0.0618 & -0.0199 \\
\hline & Max. Shear Strain* & 0.0426 & 0.0467 & 0.0041 \\
\hline & Max. Shear Stress (MPa) & $7.2 \times 10^{-6}$ & $8.2 \mathrm{e}-6$ & $1.0 \times 10^{-6}$ \\
\hline & Fluid Pressure (MPa) & 0.1190 & 0.0590 & -0.0600 \\
\hline & Total Fluid Flux (mm/s) & $2.3 \times 10^{-5}$ & $3.0 \times 10^{-6}$ & $-2.0 \times 10^{-5}$ \\
\hline & Volume Ratio* & 1.0310 & 0.9850 & -0.0460 \\
\hline \multicolumn{5}{|c|}{$\begin{array}{l}\text { *Variable is dimensionless. } \\
\text { Right far column shows how these values change from the loading to } \\
\text { relaxation phase. For } 3^{\text {rd }} \text { principal strain only, positive and negative changes } \\
\text { indicate a decrease and increase in compression respectively. }\end{array}$} \\
\hline
\end{tabular}


Table B.10: Summary of selected result variables for the peripheral superficial zone microscale model (D) of OA tissue subjected to axial quasi-static indentation.

\begin{tabular}{|c|c|c|c|c|}
\hline Region & Variable & Peak 1 & $\begin{array}{c}\text { Peak 2 } \\
(t=100 s)\end{array}$ & Peak (2-1) \\
\hline \multirow{5}{*}{ ECM } & $1^{\text {st }}$ Principal Strain* & 0.0026 & 0.0098 & 0.0072 \\
\hline & $3^{\text {rd }}$ Principal Strain* & -0.0022 & -0.0045 & -0.0023 \\
\hline & Fluid Pressure (MPa) & $-5.9 \times 10^{-4}$ & 0.0015 & 0.0021 \\
\hline & Total Fluid Flux (mm/s) & $2.1 \times 10^{-5}$ & $6.8 \times 10^{-5}$ & $4.7 \times 10^{-5}$ \\
\hline & Volume Ratio* & 1.0010 & 1.0040 & 0.0030 \\
\hline \multirow{5}{*}{ PCM } & $1^{\text {st }}$ Principal Strain* & 0.0043 & 0.0134 & 0.0091 \\
\hline & $3^{\text {rd }}$ Principal Strain* & -0.0051 & -0.0136 & -0.0085 \\
\hline & Fluid Pressure (MPa) & $-4.2 \times 10^{-4}$ & 0.0022 & 0.0026 \\
\hline & Total Fluid Flux (mm/s) & $1.5 \times 10^{-6}$ & $8.2 \times 10^{-6}$ & $6.7 \times 10^{-6}$ \\
\hline & Volume Ratio* & 0.9993 & 0.9996 & 0.0003 \\
\hline \multirow{7}{*}{ Cell } & $1^{\text {st }}$ Principal Strain* & 0.0057 & 0.0193 & 0.0136 \\
\hline & $3^{\text {rd }}$ Principal Strain* & -0.0043 & $-3.0 \times 10^{-4}$ & 0.0040 \\
\hline & Max. Shear Strain & 0.0046 & 0.0098 & 0.0052 \\
\hline & Max. Shear Stress (MPa) & $1.4 \times 10^{-6}$ & $2.9 \times 10^{-6}$ & $1.5 \times 10^{-6}$ \\
\hline & Fluid Pressure (MPa) & $-4.3 \times 10^{-4}$ & 0.0024 & 0.0028 \\
\hline & Total Fluid Flux (mm/s) & $2.8 \times 10^{-6}$ & $1.0 \times 10^{-5}$ & $7.2 \times 10^{-6}$ \\
\hline & Volume Ratio* & 1.0050 & 1.0210 & 0.0160 \\
\hline \multicolumn{5}{|c|}{$\begin{array}{l}\text { *Variable is dimensionless. } \\
\text { Right far column shows how these values change from the loading to } \\
\text { relaxation phase. For } 3^{\text {rd }} \text { principal strain only, positive and negative changes } \\
\text { indicate a decrease and increase in compression respectively. }\end{array}$} \\
\hline
\end{tabular}


Table B.11: Summary of selected result variables for the peripheral middle zone microscale model (E) of OA tissue subjected to axial quasi-static indentation.

\begin{tabular}{|c|c|c|c|c|}
\hline Region & Variable & Peak 1 & $\begin{array}{c}\text { Peak } 2 \\
(t=100 s)\end{array}$ & Peak (2-1) \\
\hline \multirow{5}{*}{ ECM } & $1^{\text {st }}$ Principal Strain* & 0.0032 & 0.0029 & $-3.0 \times 10^{-4}$ \\
\hline & $3^{\text {rd }}$ Principal Strain* & -0.0040 & -0.0024 & 0.0016 \\
\hline & Fluid Pressure (MPa) & 0.0071 & 0.0119 & 0.0048 \\
\hline & Total Fluid Flux (mm/s) & $4.9 \times 10^{-5}$ & $6.2 \times 10^{-5}$ & $1.3 \times 10^{-5}$ \\
\hline & Volume Ratio* & 1.0010 & 1.0020 & 0.0010 \\
\hline \multirow{5}{*}{ PCM } & $1^{\text {st }}$ Principal Strain* & 0.0063 & 0.0042 & -0.0021 \\
\hline & $3^{\text {rd }}$ Principal Strain* & -0.0070 & -0.0043 & 0.0027 \\
\hline & Fluid Pressure (MPa) & 0.0075 & 0.0124 & 0.0049 \\
\hline & Total Fluid Flux (mm/s) & $4.6 \times 10^{-6}$ & $5.4 \times 10^{-6}$ & $8.0 \times 10^{-7}$ \\
\hline & Volume Ratio* & 0.9994 & 1.0010 & 0.0016 \\
\hline \multirow{7}{*}{ Cell } & $1^{\text {st }}$ Principal Strain* & 0.0092 & 0.0083 & $-9.0 \times 10^{-4}$ \\
\hline & $3^{\text {rd }}$ Principal Strain* & -0.0080 & -0.0044 & 0.0036 \\
\hline & Max. Shear Strain* & 0.0082 & 0.0063 & -0.0019 \\
\hline & Max. Shear Stress (MPa) & $1.4 \times 10^{-6}$ & $1.1 \times 10^{-6}$ & $-3.0 \times 10^{-7}$ \\
\hline & Fluid Pressure (MPa) & 0.0075 & 0.0125 & 0.0050 \\
\hline & Total Fluid Flux (mm/s) & $7.5 \times 10^{-6}$ & $1.1 \times 10^{-5}$ & $3.5 \times 10^{-6}$ \\
\hline & Volume Ratio* & 1.0080 & 1.0100 & 0.0020 \\
\hline \multicolumn{5}{|c|}{$\begin{array}{l}\text { *Variable is dimensionless. } \\
\text { Right far column shows how these values change from the loading to } \\
\text { relaxation phase. For } 3^{\text {rd }} \text { principal strain only, positive and negative changes } \\
\text { indicate a decrease and increase in compression respectively. }\end{array}$} \\
\hline
\end{tabular}


Table B.12: Summary of selected result variables for the peripheral deep zone microscale model (F) of OA tissue subjected to axial quasi-static indentation.

\begin{tabular}{|c|c|c|c|c|}
\hline Region & Variable & Peak 1 & $\begin{array}{c}\text { Peak } 2 \\
(t=100 s)\end{array}$ & Peak (2-1) \\
\hline \multirow{5}{*}{ ECM } & $1^{\text {st }}$ Principal Strain* & 0.0046 & 0.0048 & $2.0 \times 10^{-4}$ \\
\hline & $3^{\text {rd }}$ Principal Strain* & -0.0069 & -0.0069 & 0.0000 \\
\hline & Fluid Pressure (MPa) & 0.0134 & 0.0185 & 0.0051 \\
\hline & Total Fluid Flux (mm/s) & $7.6 \times 10^{-5}$ & $6.9 \times 10^{-5}$ & $-7.0 \times 10^{-6}$ \\
\hline & Volume Ratio* & 0.9996 & 0.9996 & 0.0000 \\
\hline \multirow{5}{*}{ PCM } & $1^{\text {st }}$ Principal Strain* & 0.0049 & 0.0054 & $5.0 \times 10^{-4}$ \\
\hline & $3^{\text {rd }}$ Principal Strain* & -0.0125 & -0.0133 & $-8.0 \times 10^{-4}$ \\
\hline & Fluid Pressure (MPa) & 0.0135 & 0.0186 & 0.0051 \\
\hline & Total Fluid Flux (mm/s) & $3.4 \times 10^{-6}$ & $3 \times 10^{-6}$ & $-4.0 \times 10^{-7}$ \\
\hline & Volume Ratio* & 0.9958 & 0.9959 & $1.0 \times 10^{-4}$ \\
\hline \multirow{7}{*}{ Cell } & $1^{\text {st }}$ Principal Strain* & 0.0132 & 0.0132 & 0.0000 \\
\hline & $3^{\text {rd }}$ Principal Strain* & -0.0118 & -0.0122 & $-4.0 \times 10^{-4}$ \\
\hline & Max. Shear Strain* & 0.0128 & 0.0127 & $-1.0 \times 10^{-4}$ \\
\hline & Max. Shear Stress (MPa) & $2.2 \times 10^{-6}$ & $2.1 \times 10^{-6}$ & $-1.0 \times 10^{-7}$ \\
\hline & Fluid Pressure (MPa) & 0.0135 & 0.0186 & 0.0051 \\
\hline & Total Fluid Flux (mm/s) & $1.1 \times 10^{-5}$ & $1.2 \times 10^{-5}$ & $1.0 \times 10^{-6}$ \\
\hline & Volume Ratio* & 1.0060 & 1.0060 & 0.0000 \\
\hline \multicolumn{5}{|c|}{$\begin{array}{l}\text { *Variable is dimensionless. } \\
\text { Right far column shows how these values change from the loading to } \\
\text { relaxation phase. For } 3^{\text {rd }} \text { principal strain only, positive and negative changes } \\
\text { indicate a decrease and increase in compression respectively. }\end{array}$} \\
\hline
\end{tabular}




\section{Appendix C : Miscellaneous}

\section{C.1 Mesh Sensitivity Analysis}

Table C.1: Influence of mesh refinement in the initial macroscale finite element cartilage model. Mesh refinement resulted in minor differences in the maximum fluid pressure.

\begin{tabular}{cccc}
\hline Model (Number of elements) & $\begin{array}{c}\text { Element size } \\
(\mathrm{mm})\end{array}$ & $\begin{array}{c}\text { Maximum fluid pressure } \\
(\mathrm{MPa})\end{array}$ & $\begin{array}{c}\text { Error } \\
(\%)\end{array}$ \\
\hline $1(40)$ & 0.5 & 3.01 & - \\
$2(160)$ & 0.25 & 2.58 & 14.28 \\
$3(640)$ & 0.125 & 2.87 & 11.24 \\
$4(2560)$ & 0.0625 & 2.84 & 1.04 \\
$5(10240)$ & 0.03125 & 2.83 & 0.35 \\
\hline
\end{tabular}

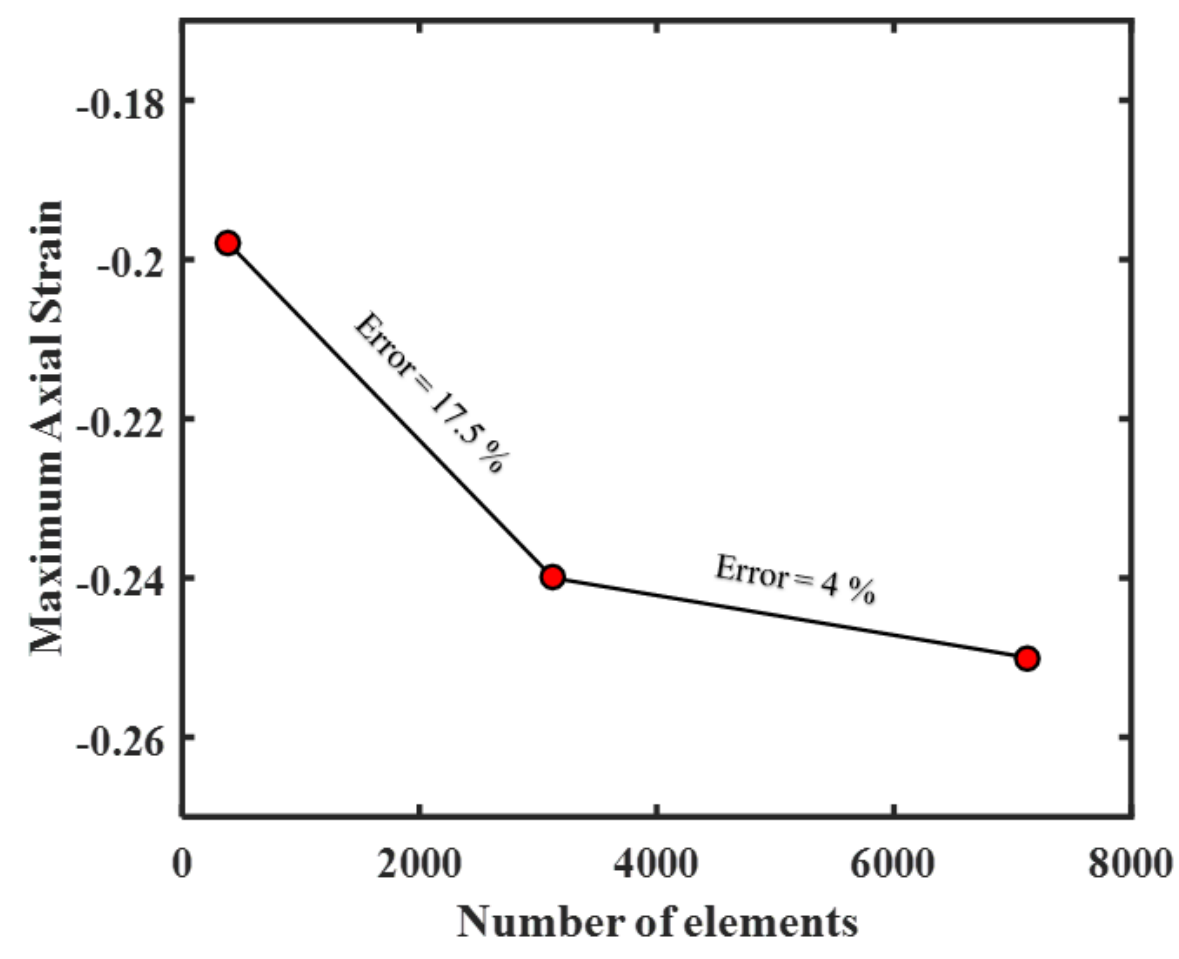

Figure C.1: Influence of mesh refinement in the microscale finite element cartilage models. Mesh refinement beyond 3136 elements resulted in minor differences in axial strain ( $4 \%$ error). 


\section{C.2 Multiscale Homogenization and Deformed Outlines of Selected Models}

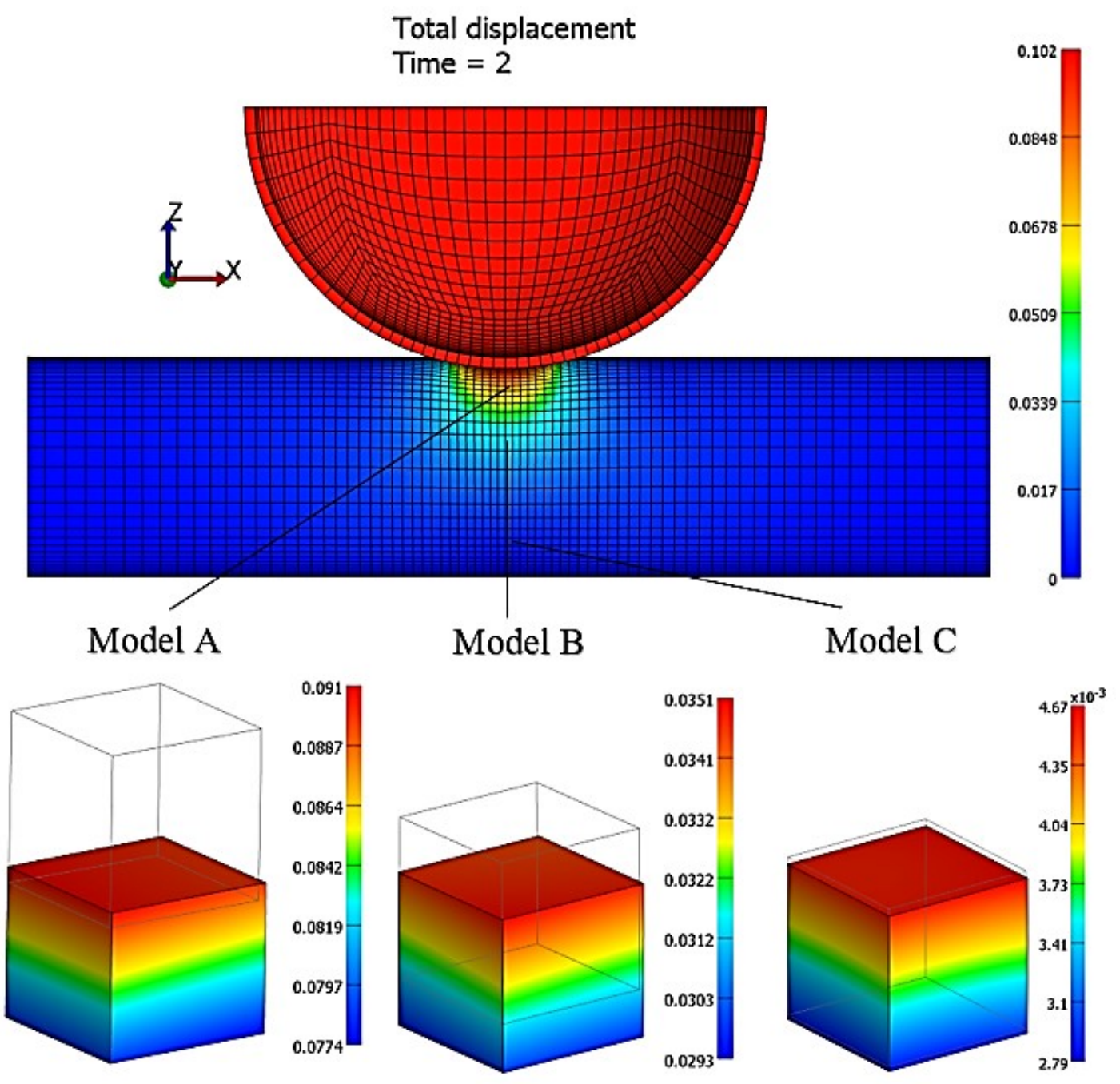

Figure C.2: A comparison of undeformed and deformed outlines of healthy central microscale models (A-C) at peak ramp load applied to macroscale model at 2 seconds. Contour plots show consistent displacements between both scales. 


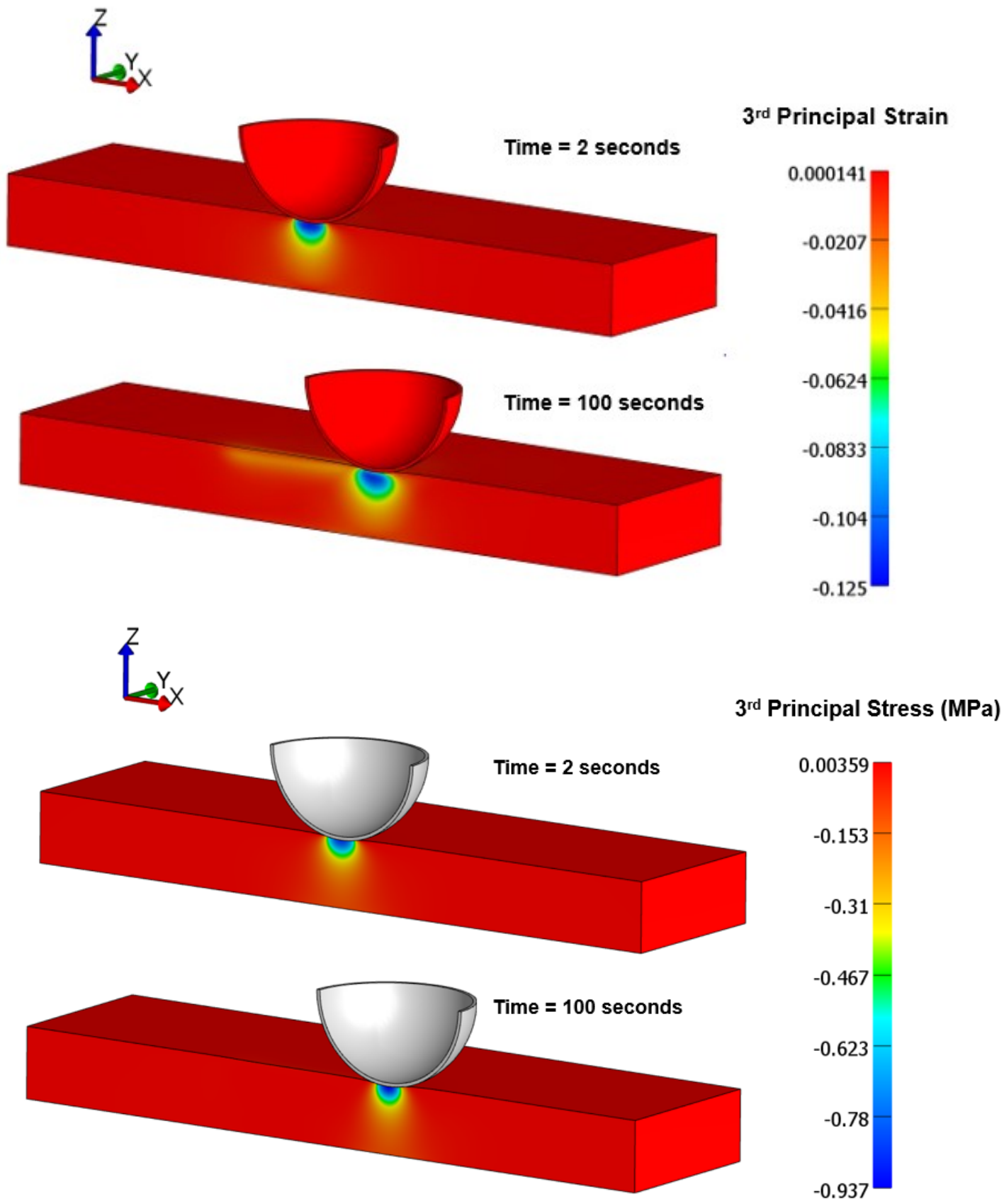

Figure C.3: Contour plots of $3^{\text {rd }}$ principal strain (top) and stress (bottom) in macroscale cartilage at the end of ramp load ( 2 seconds) and 100 seconds under the novel and physiological cyclic sliding indentation. 


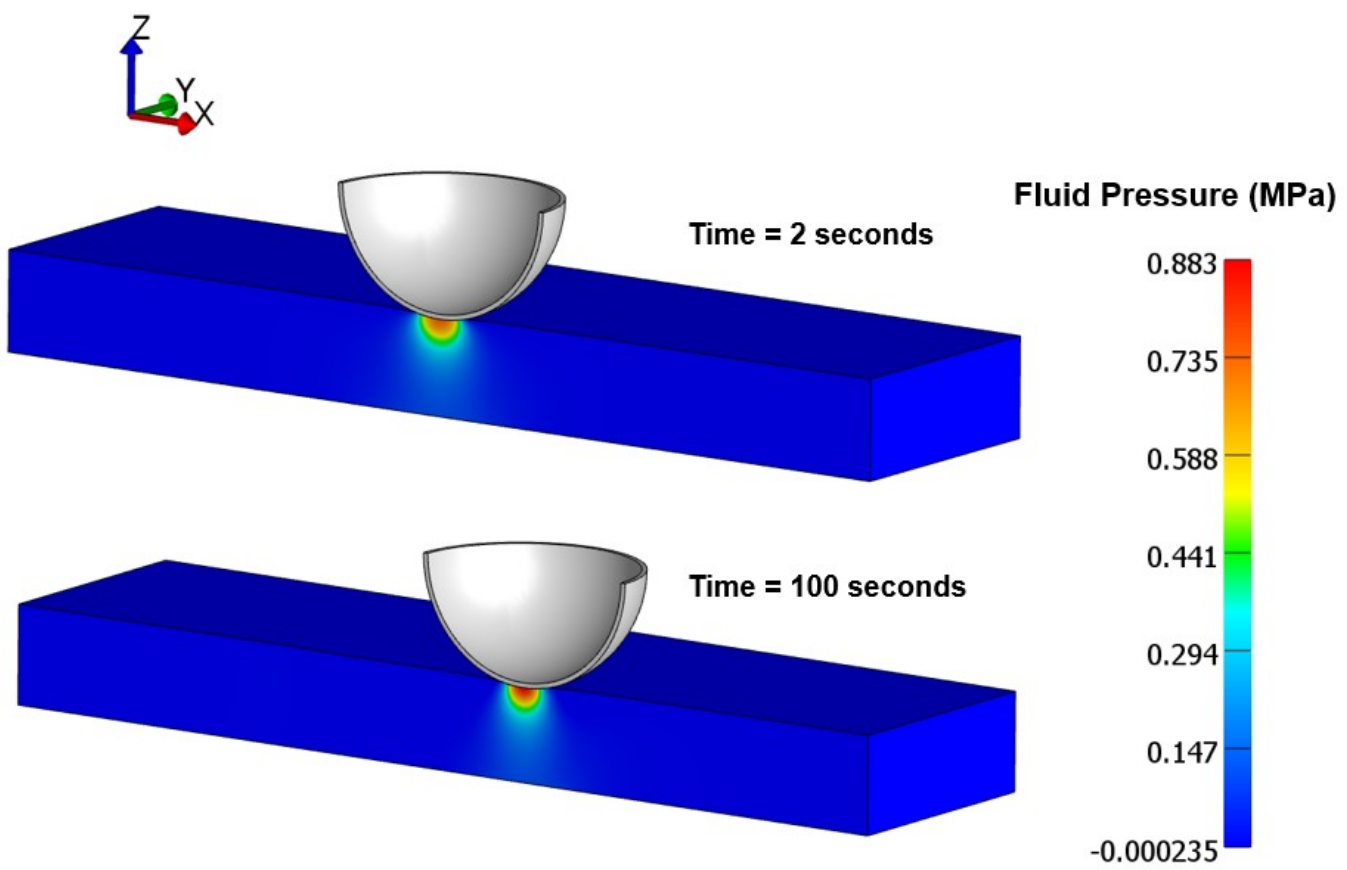

Figure C.4: Contour plots of fluid pressurization in macroscale cartilage at the end of ramp load ( 2 seconds) and 100 seconds under the novel and physiological cyclic sliding indentation. 


\section{C.4 Additional Time Plots for Healthy Cartilage Under Various Loads}
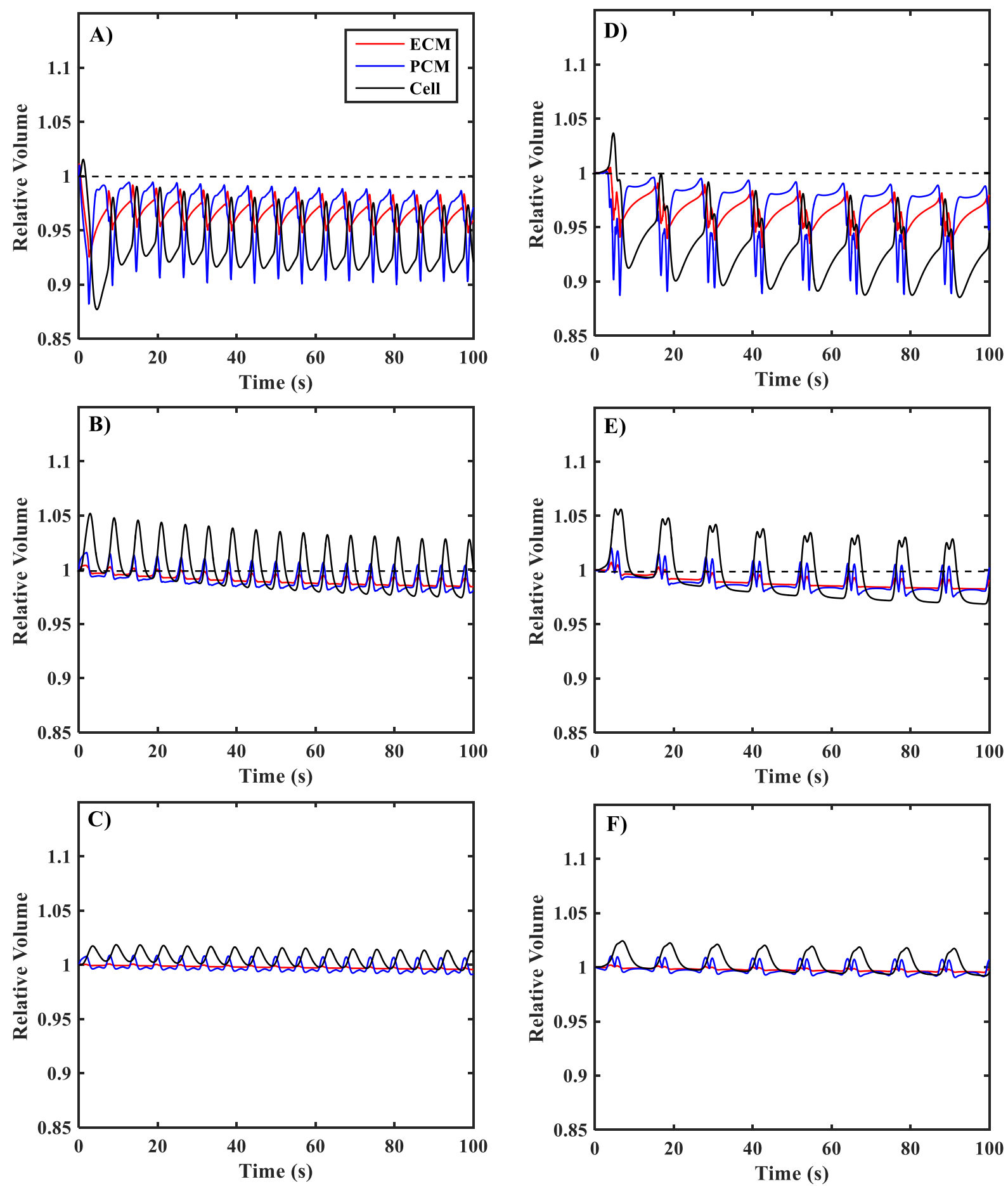

Figure C.5: Relative volume in the microenvironment of healthy cartilage at the superficial, middle, and deep zones of the central (A-C), and peripheral (D-F) locations under cyclic sliding indentation. 

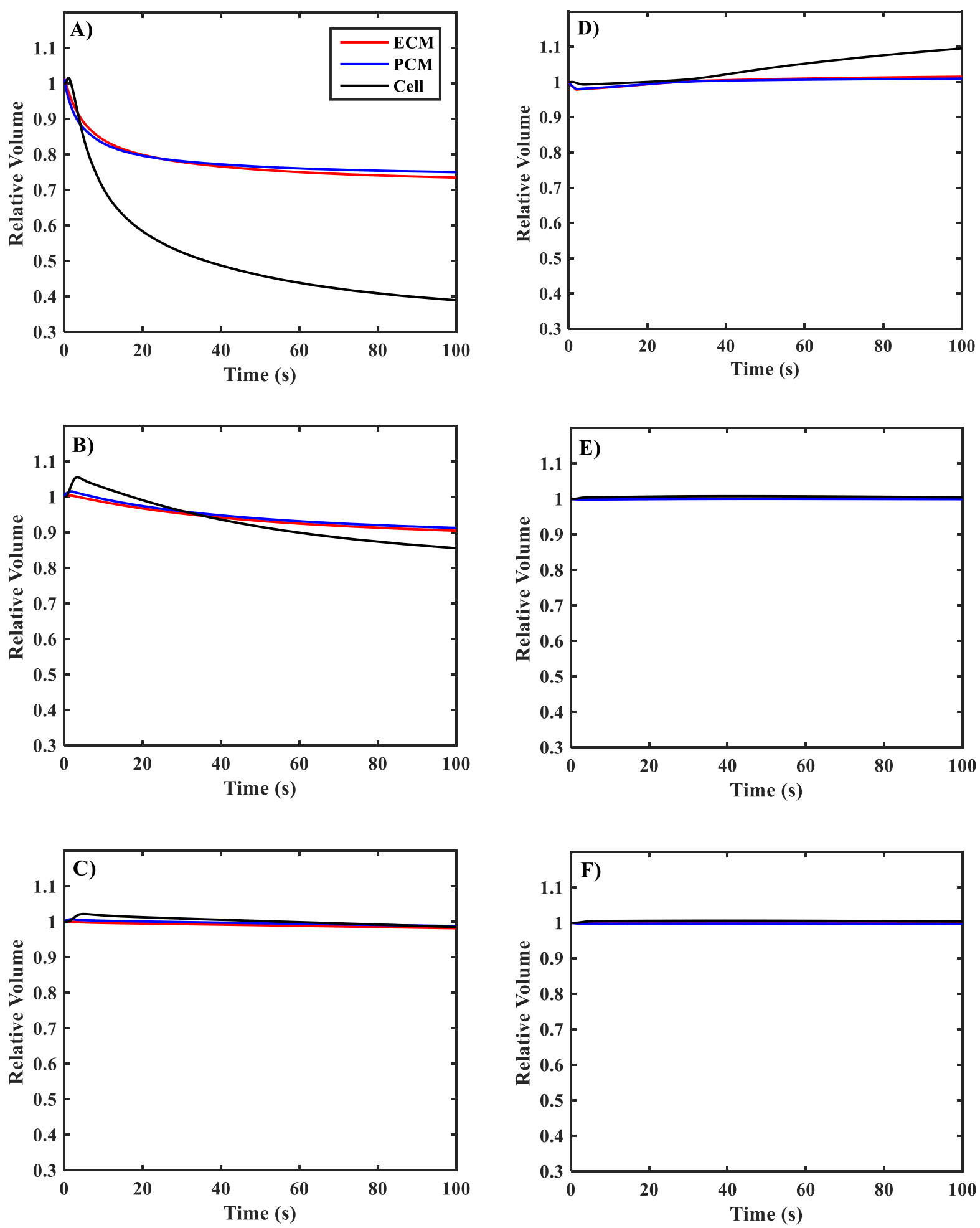

Figure C.6: Relative volume in the microenvironment of healthy cartilage at the superficial, middle, and deep zones of the central (A-C), and peripheral (D-F) locations under axial quasi-static indentation. 


\section{Cyclic Sliding Indentation}

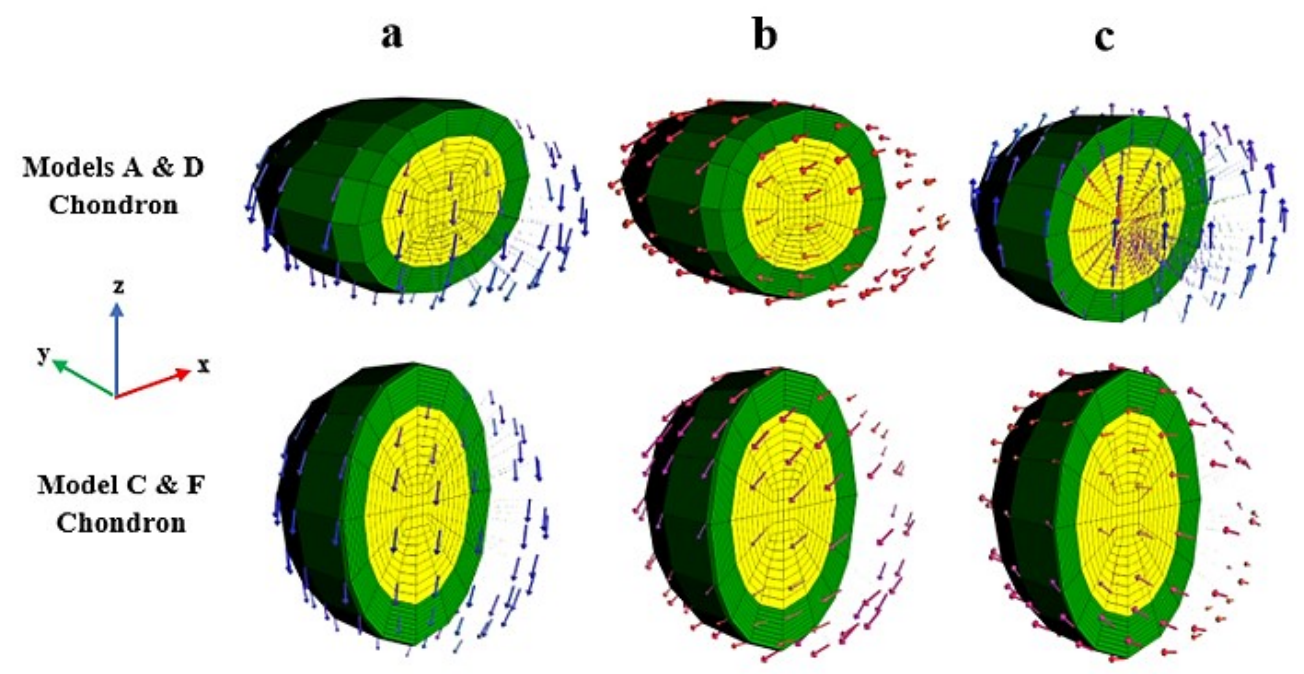

Figure C.7: Fluid flux vector plots for selected models subjected to cyclic sliding indentation. Microscale models in the middle zone (B and E) are not shown since they had similar flux patterns to superficial zone (A and D). In all microscale models, the flux is directed along -z-direction during their respective loading phase (a). The flux was generally directed along the $\mathrm{x}$-axis in the direction opposite to the sliding direction of the indenter (b). During the unloaded phases (c), the flux was generally directed in the $+z-$ direction for the microscale models in the superficial zone (A and D) and middle zone (B and $\mathrm{E})$. Whereas, the flux was generally directed along the $\mathrm{x}$-axis for microscale models in the deep zone ( $\mathrm{C}$ and $\mathrm{F}$ ) depending on the relative $\mathrm{x}$-position of the indenter. 


\section{Axial Quasi-Static Indentation}

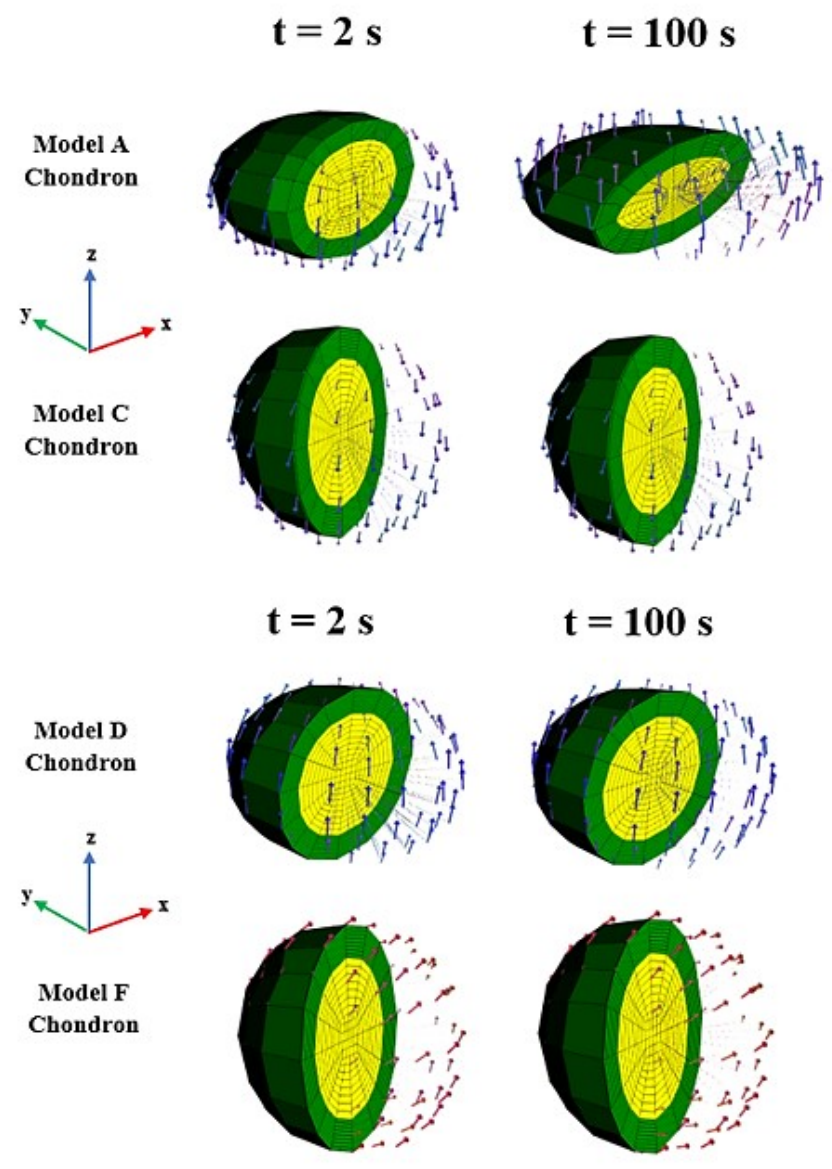

Figure C.8: Fluid flux vector plots for selected models subjected to axial quasi-static indentation. Microscale models in the middle zone (B and E) are not shown since they had similar flux patterns to microscale models $A$ and $D$. In microscale models A-C, the flux is directed along - $\mathrm{z}$-direction at the end of the peak ramp load ( $\mathrm{t}=2 \mathrm{~seconds})$. At constant load held for 100 seconds, the flux direction changes to +z-direction in microscale models A and B (not shown). However, in microscale model C, the flux direction remains in the -z-direction. In the peripheral locations, the flux vector in microscale models D and E (not shown) always remained in the +z-direction over 100 seconds. Whereas, the flux vector in microscale model $\mathrm{F}$ always remained in the $+\mathrm{x}-$ direction over 100 seconds. 


\section{C.5 Additional Time Plots for OA Cartilage Under Various Loads}
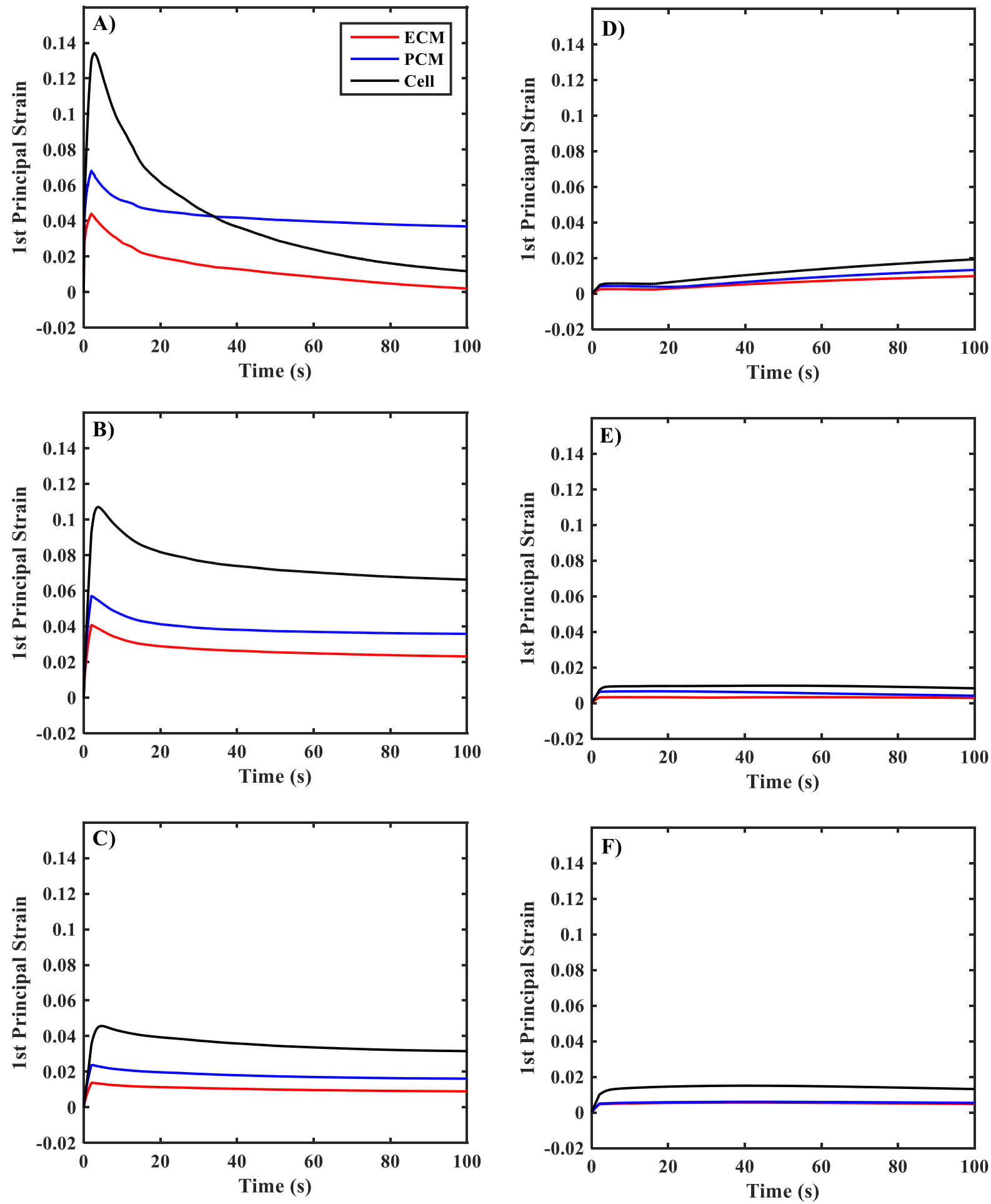

Figure C.9: First principal strain in the microenvironment of OA cartilage at the superficial, middle, and deep zones of the central (A-C), and peripheral (D-F) locations under axial quasi-static indentation. 

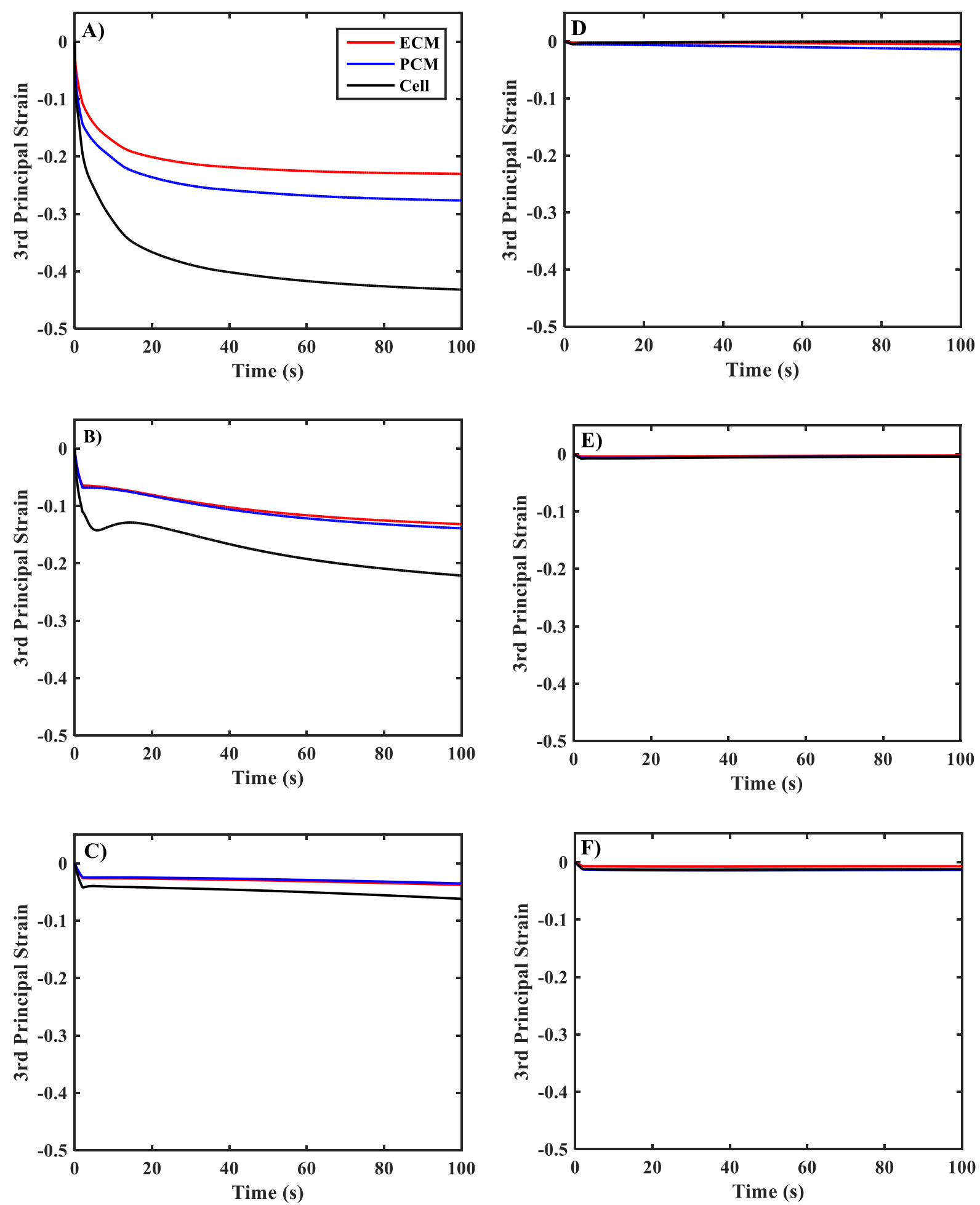

Figure C.10: Third principal strain in the microenvironment of OA cartilage at the superficial, middle, and deep zones of the central (A-C), and peripheral (D-F) locations under axial quasi-static indentation 

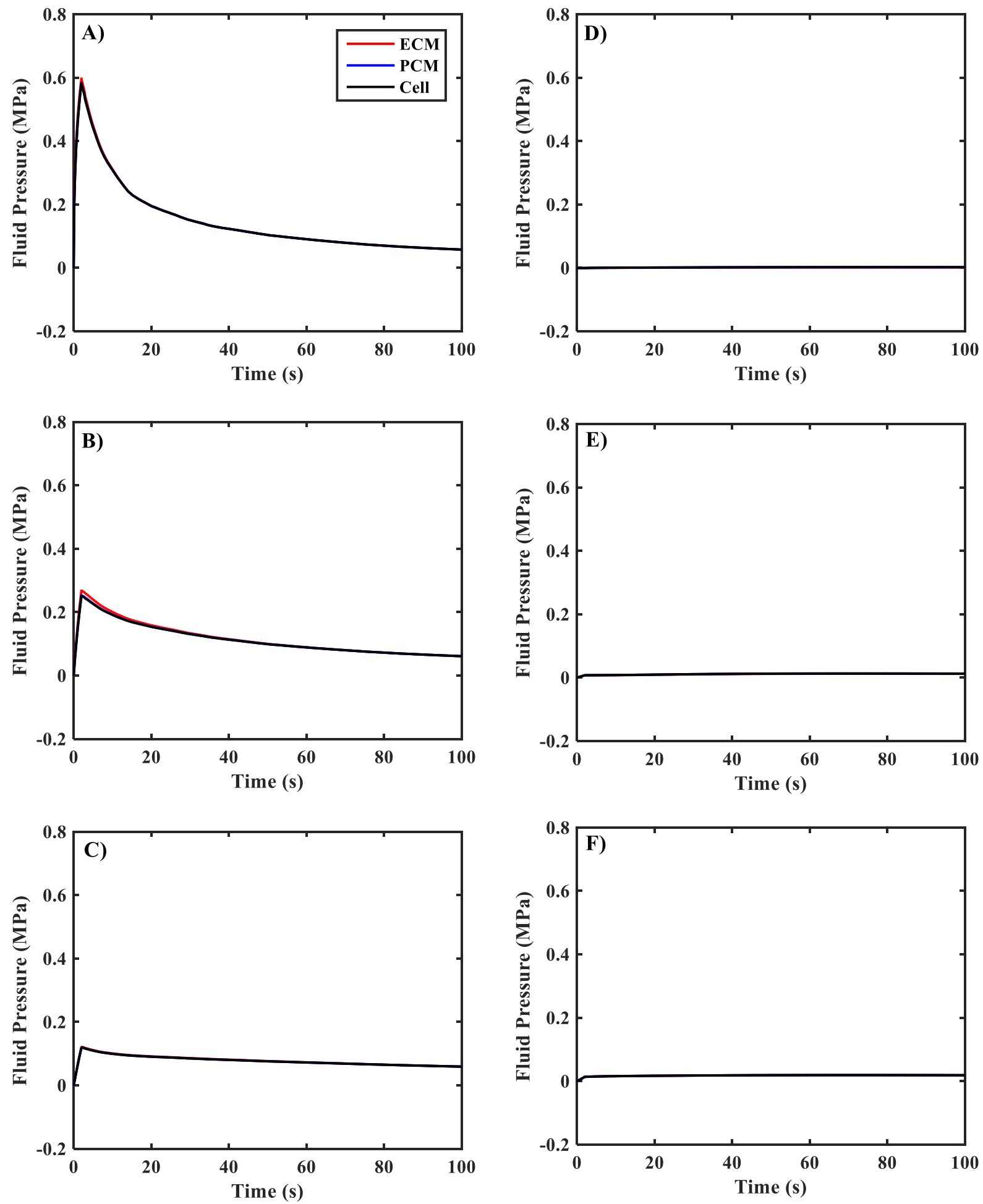

Figure C.11: Fluid pressure in the microenvironment of OA cartilage at the superficial, middle, and deep zones of the central (A-C), and peripheral (D-F) locations under axial quasi-static indentation. 

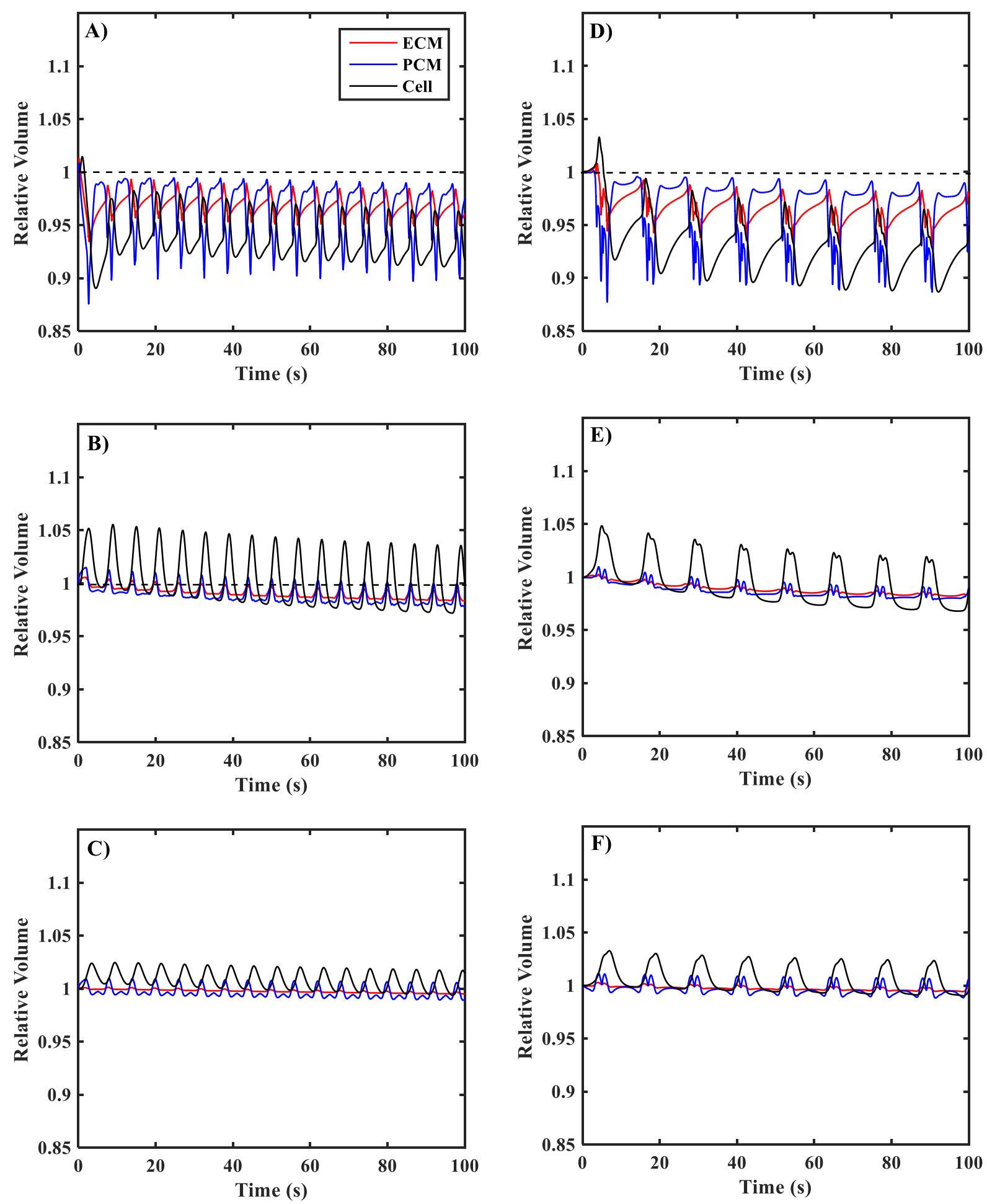

Figure C.12: Relative volume in the microenvironment of OA cartilage at the superficial, middle, and deep zones of the central (A-C), and peripheral (D-F) locations under cyclic sliding indentation. 


\section{C.6 MATLAB Script (pseudo code)}

$\%$ Cartilage Multiscale Analysis

\% Author: Abdul R. Khan, B.Eng. (E.I.T)

\% Email: abdulrkhan3@cmail.carleton.ca

$\%$ Student ID: 100889821

\% Institution: Carleton University

$\% \%$ General Information of Macro- and Microscale Models

$\%$ "N" denotes macroscale nodal values

$\%$ "n" denotes microscale nodal values

$\%$ All (hexahedral) elements extracted from the macroscale model contains nodes 1 to 4 on the bottom face, and nodes 5 to 8 on the top face (counter-clockwise numbering scheme)

$\%$ Positive coordinate axes are defined such that $\mathrm{x}, \mathrm{y}$, and $\mathrm{z}$ are (right, into the page, and up) respectively

$\% \%$ Nomenclature

$\% \mathrm{X} \#, \mathrm{Y} \#, \mathrm{Z} \#$ are position vectors in $(\mathrm{mm})$

$\% \mathrm{~N} \# \mathrm{X}, \mathrm{N} \# \mathrm{Y}, \mathrm{N} \# \mathrm{Z}$ are $\mathrm{x}, \mathrm{y}$, and $\mathrm{z}$ displacements in $(\mathrm{mm})$

$\% \mathrm{~N} \# \mathrm{~F}$ denotes effective fluid pressure in $(\mathrm{MPa})$

\% “\#” ranges from 1 to 8 for the 8 corner nodes of the hexahedral (brick) element

$\% \%$ Method

$\%$ Prompt user to input the extracted position vectors from the macroscale model volume element of interest ensuring the numbering scheme (above) is followed. Perform this for each zone (superficial, middle, and deep) at central and peripheral locations of the tissue.

*User can perform this for more locations, however only the 6 distinct locations were investigated in this thesis, as described in Chapter 3.

$\%$ Store all position vectors in appropriate variables

$$
\begin{aligned}
& \text { N1 }=[X 1, \mathrm{Y} 1, \mathrm{Z1}] ; \\
& \mathrm{N} 2=[\mathrm{X} 2, \mathrm{Y} 2, \mathrm{Z} 2] ; \\
& \mathrm{N} 3=[\mathrm{X} 3, \mathrm{Y3}, \mathrm{Z} 3] ; \\
& \mathrm{N} 4=[\mathrm{X} 4, \mathrm{Y} 4, \mathrm{Z} 4] ; \\
& \mathrm{N} 5=[\mathrm{X} 5, \mathrm{Y} 5, \mathrm{Z} 5] ; \\
& \mathrm{N} 6=[\mathrm{X} 6, \mathrm{Y} 6, \mathrm{Z} 6] ;
\end{aligned}
$$


$\mathrm{N} 7=[\mathrm{X} 7, \mathrm{Y} 7, \mathrm{Z7}] ;$

$\mathrm{N} 8=[\mathrm{X} 8, \mathrm{Y} 8, \mathrm{Z} 8] ;$

$\%$ Determine isoparametric (natural) coordinates of the microscale model with respect to the macroscale model. Note: Natural coordinates are denoted by the conventional Greek letters $(\Psi, \eta, \zeta)$ as (psi, eta, zeta) respectively.

The user must have one common node to relate both scales, in this study $(\mathrm{N} 1=\mathrm{n} 1)$

$\%$ Since the desired volume of all micro-scale models is constrained to $(100 \times 100 \times 100)$ microns, the user can define natural coordinates as follows;

$\mathrm{n} 1=\mathrm{N} 1 ; \%$ Common node relating both scales

$\mathrm{n} 2=[\mathrm{N} 1(1,1)+0.1,0, \mathrm{~N} 1(1,3)]$

$\mathrm{n} 3=[\mathrm{N} 1(1,1)+0.1,0.1, \mathrm{~N} 1(1,3)]$;

$\mathrm{n} 4=[\mathrm{N} 1(1,1), 0.1, \mathrm{~N} 1(1,3)]$

$\mathrm{n} 5=[\mathrm{N} 1(1,1), 0, \mathrm{~N} 1(1,3)+0.1]$

$\mathrm{n} 6=[\mathrm{N} 1(1,1)+0.1,0, \mathrm{~N} 1(1,3)+0.1]$

$\mathrm{n} 7=[\mathrm{N} 1(1,1)+0.1,0.1, \mathrm{~N} 1(1,3)+0.1]$

$\mathrm{n} 8=[\mathrm{N} 1(1,1), 0.1, \mathrm{~N} 1(1,3)+0.1]$

$\% \%$ Application of Boundary Conditions (BC's)

$\%$ Using isoparametric formulation allows the use of the same shape functions used for geometry to also calculate field variables. Therefore, prompt user to input extracted boundary conditions (BC's) from each of the 8 corner nodes of the hexahedral macromodel element of interest, these are the $\mathrm{x}, \mathrm{y}, \mathrm{z}$ displacements, and effective fluid pressure.

$\%$ Linear interpolation is then required to match $\mathrm{BC}$ 's for the desired geometry of the microscale model.

$\%$ Corner Node 1 of micro-scale (n1) is an exception since it is identical to N1 of the macro-scale (sharing the same BC's) the user can simply equate all numerical values stored in rows, and columns of N1 of macro-model.

$\mathrm{x} 1$ corrected $=\mathrm{N} 1 \mathrm{X}(::)$;

Y1_corrected $=\operatorname{N1Y}(:,:)$;

z1 corrected $=\operatorname{N1Z}(:,:)$;

f1_corrected $=\operatorname{N1F}(:,:)$;

$\%$ Since the corrected field variables are time-dependent, they must also include the corresponding time column vector. Note: these time vectors differ in length due to the variation in step size used.

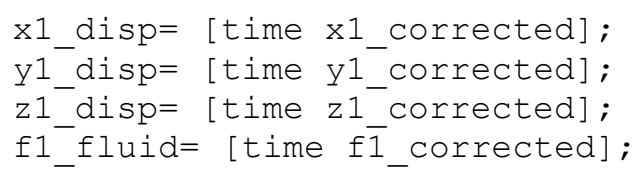


$\%$ For all other corner and surface nodes, the user must use shape functions formulae (linear in this case) which include the corresponding position vectors. For time-dependent field variables, this is done by the use of a for loop which calculates the corrected BC's for each entry in the row vector.

$\%$ Corner node 2 is shown as an example

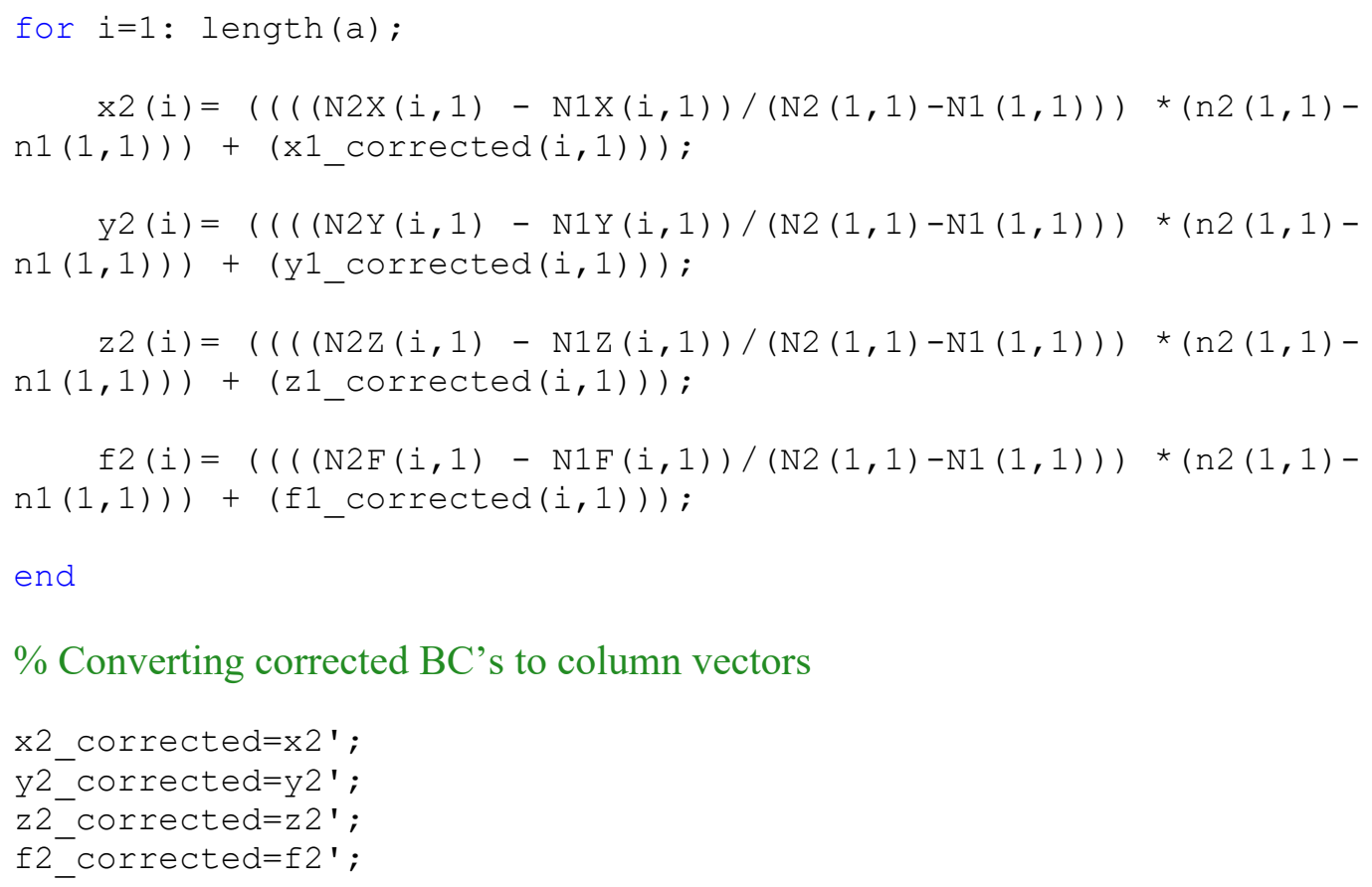

$\%$ Since the corrected field variables are time-dependent, they must also include the corresponding time column vector.

$x_{2}$ disp $=$ [time $x_{2}$ _corrected];

$\mathrm{y}^{2}$ disp $=$ [time y2 corrected];

z2_disp= [time z2_corrected];

f2_fluid=[time $f \overline{2} \_$corrected];

\% This process can be repeated for all the corner and surface nodes of the microscale mesh. In this study, once the BC's for the corner nodes were determined using linear interpolation, the BC's for the remaining surface nodes were a result of the mean of these values.

\section{$\% \%$ Preparing .txt document compatible with FEBio2.5 input files}

$\%$ Once all interpolated BC's are calculated, prepare a .txt file that is compatible with FEBio input files.

$\%$ Superficial zone of healthy tissue subjected to sliding indentation is given as an example 
fileID = fopen('Superficial_Healthy_Sliding_Center.txt','w');

\% User can reproduce the formatting using "fprintf” functions in MATLAB and store the appropriate corrected time-dependent boundary conditions as follows;

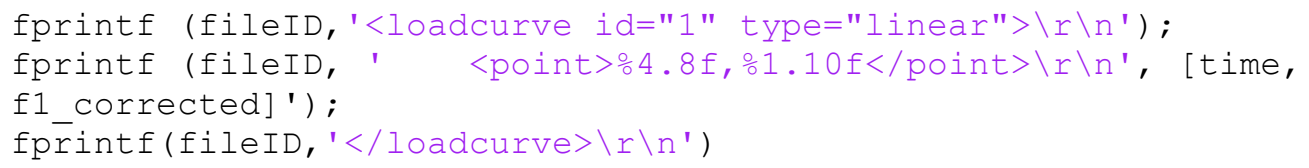

$\%$ User can repeat for ' $\mathrm{x}$ ' number of load-curves (i.e. boundary conditions) without manually prescribing them in FEBio2.6.2 pre-processor (PreView). 


\section{C.7 FEBio2.5 Input Files (pseudo code)}

$\%$ Input file for Model A (central location of the superficial zone) of OA tissue subjected to cyclic sliding indentation. All other (23) models follow same file formatting.

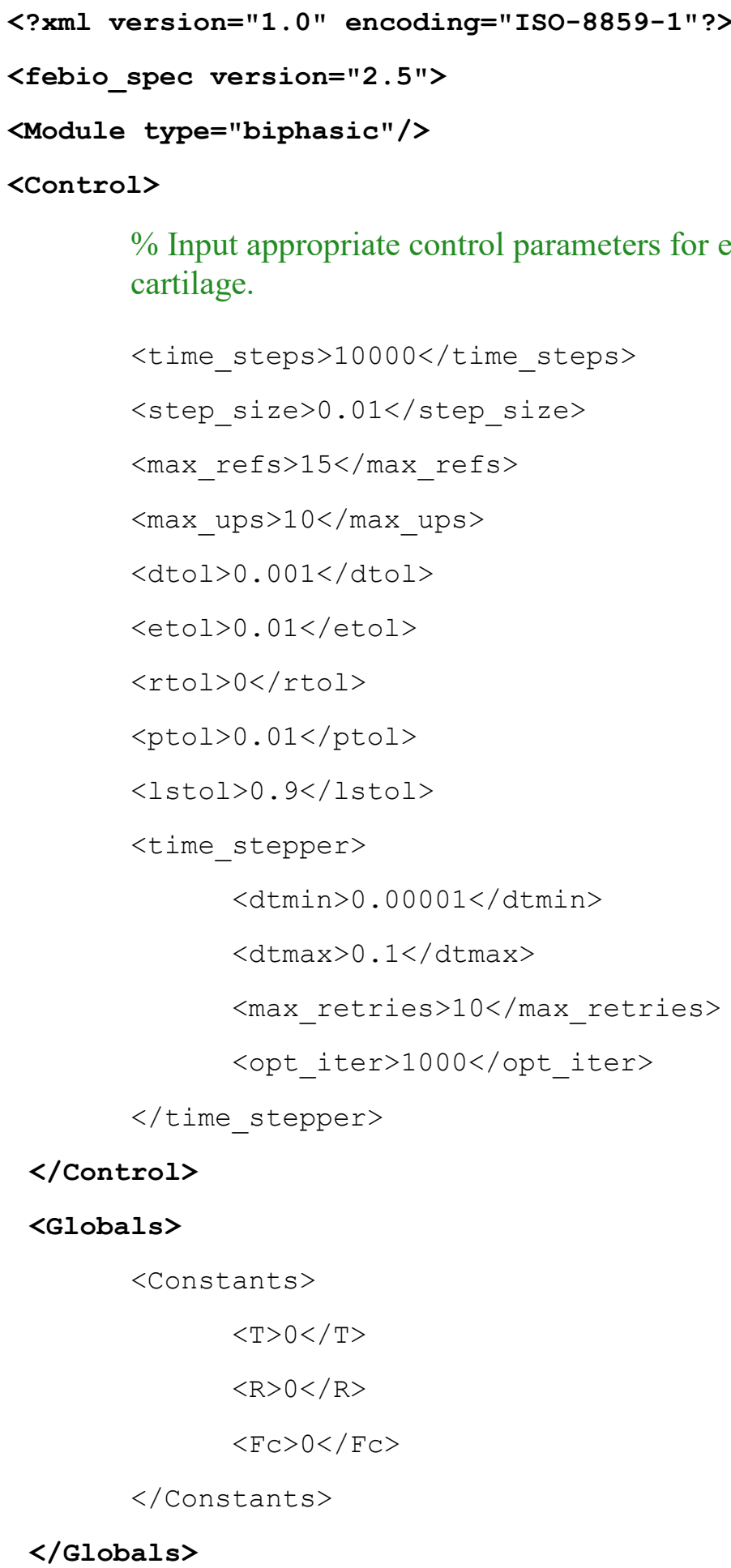


$\%$ Assign appropriate material properties

\section{$\langle$ Material>}

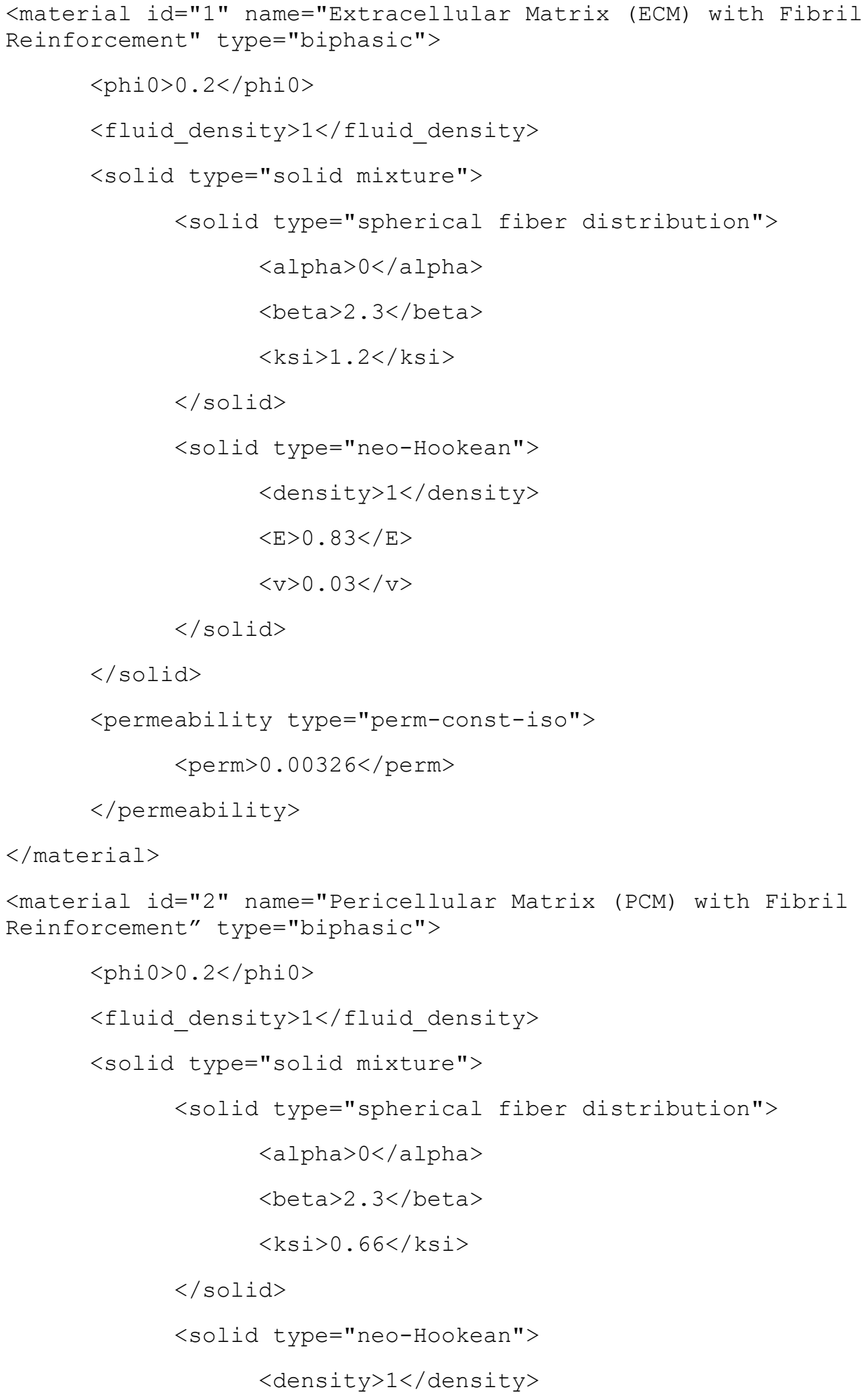




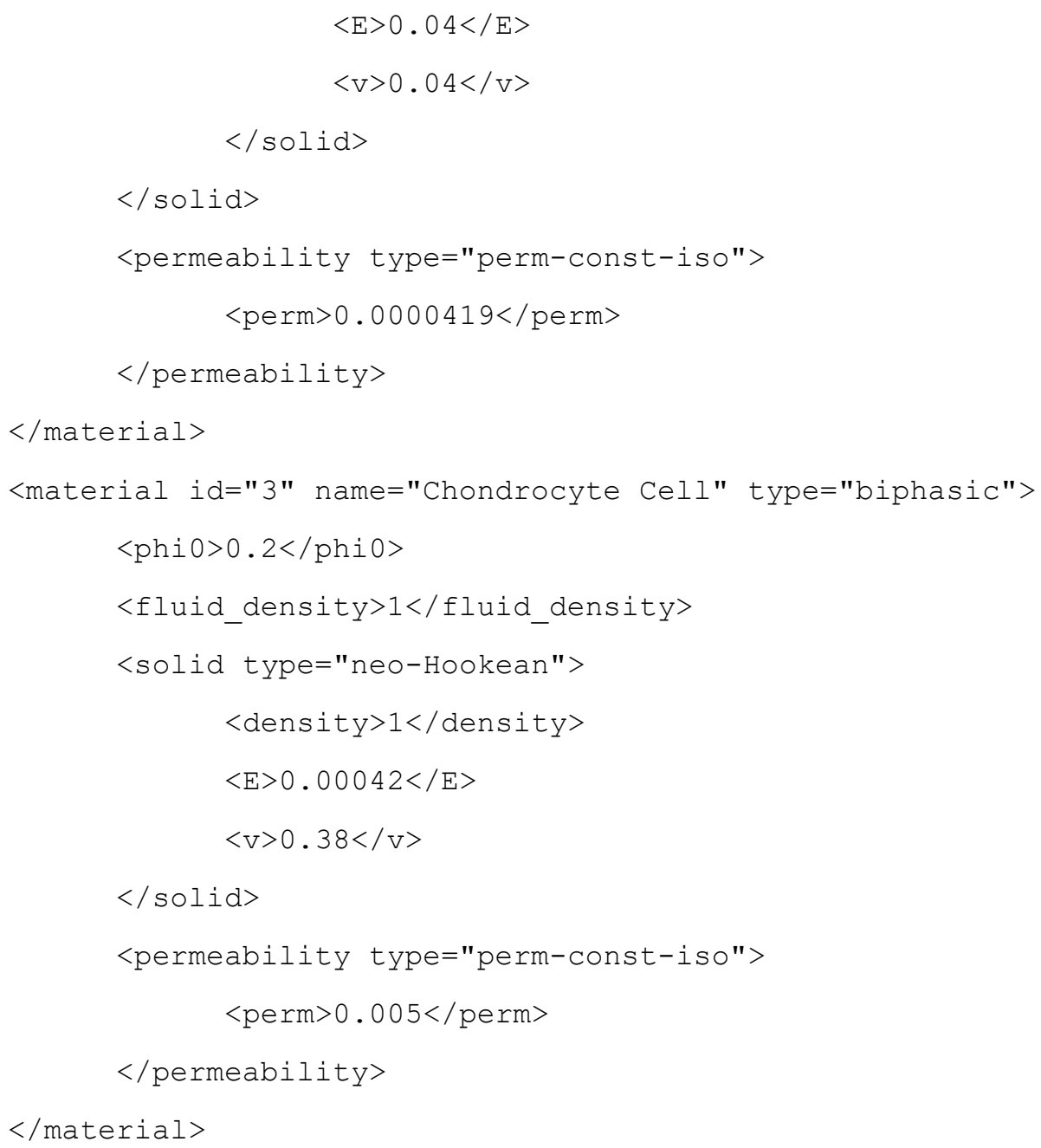

$\%$ Repeated for all fluid pressures and $\mathrm{x}, \mathrm{y}, \mathrm{z}$ displacements for a total of 392 prescribed boundary conditions. 


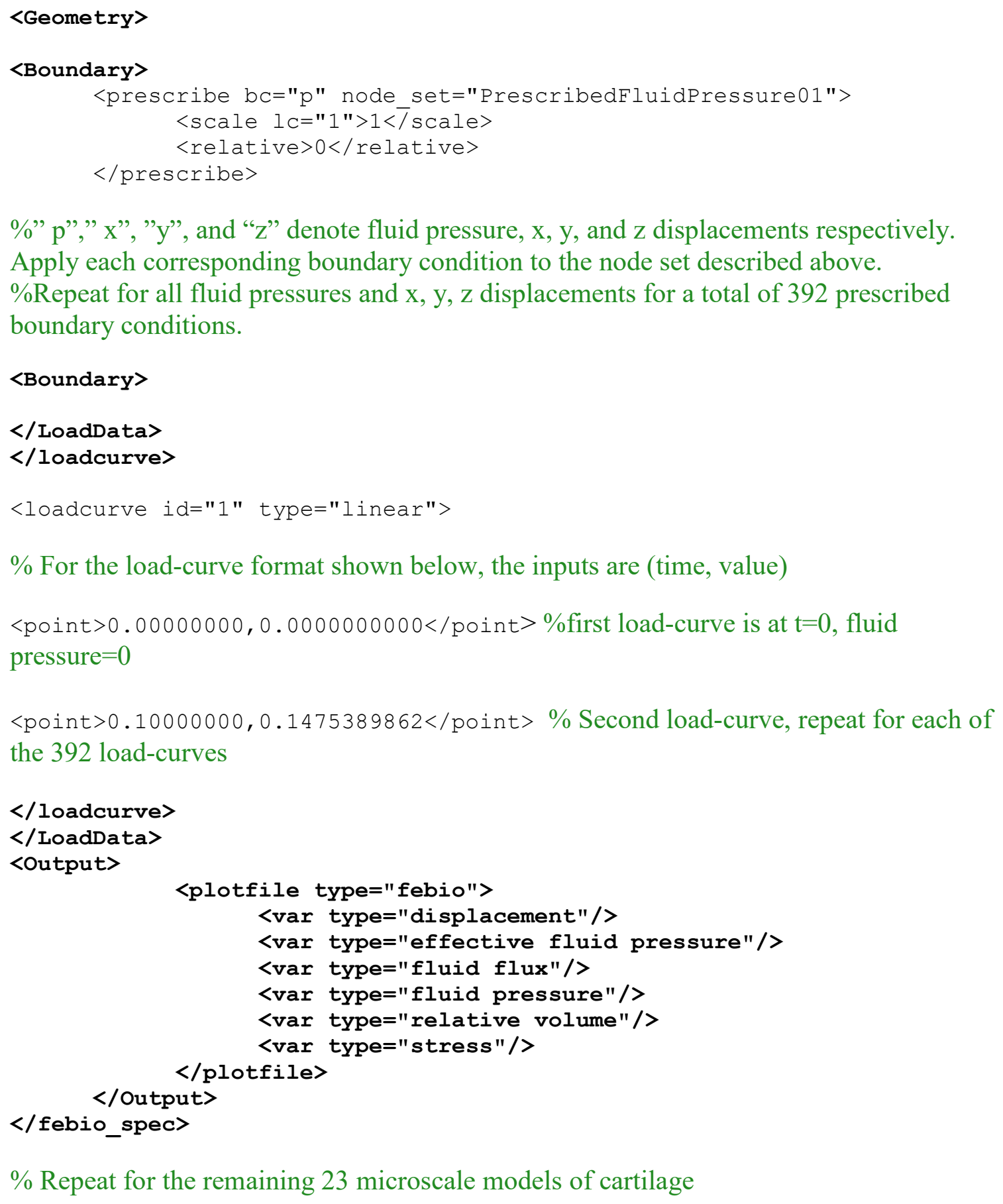

$\%$ Repeat for the remaining 23 microscale models of cartilage 